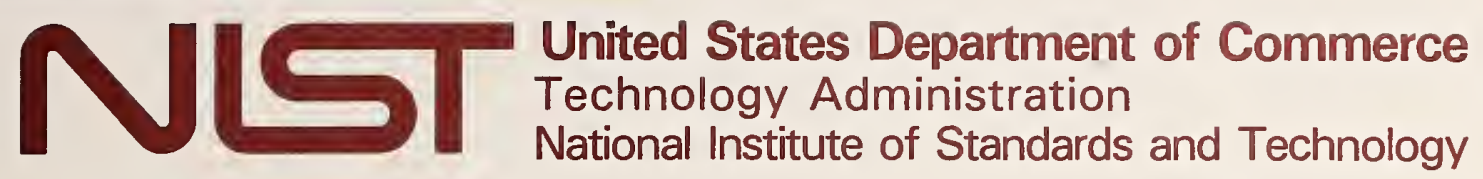

NIST Technical Note 1375

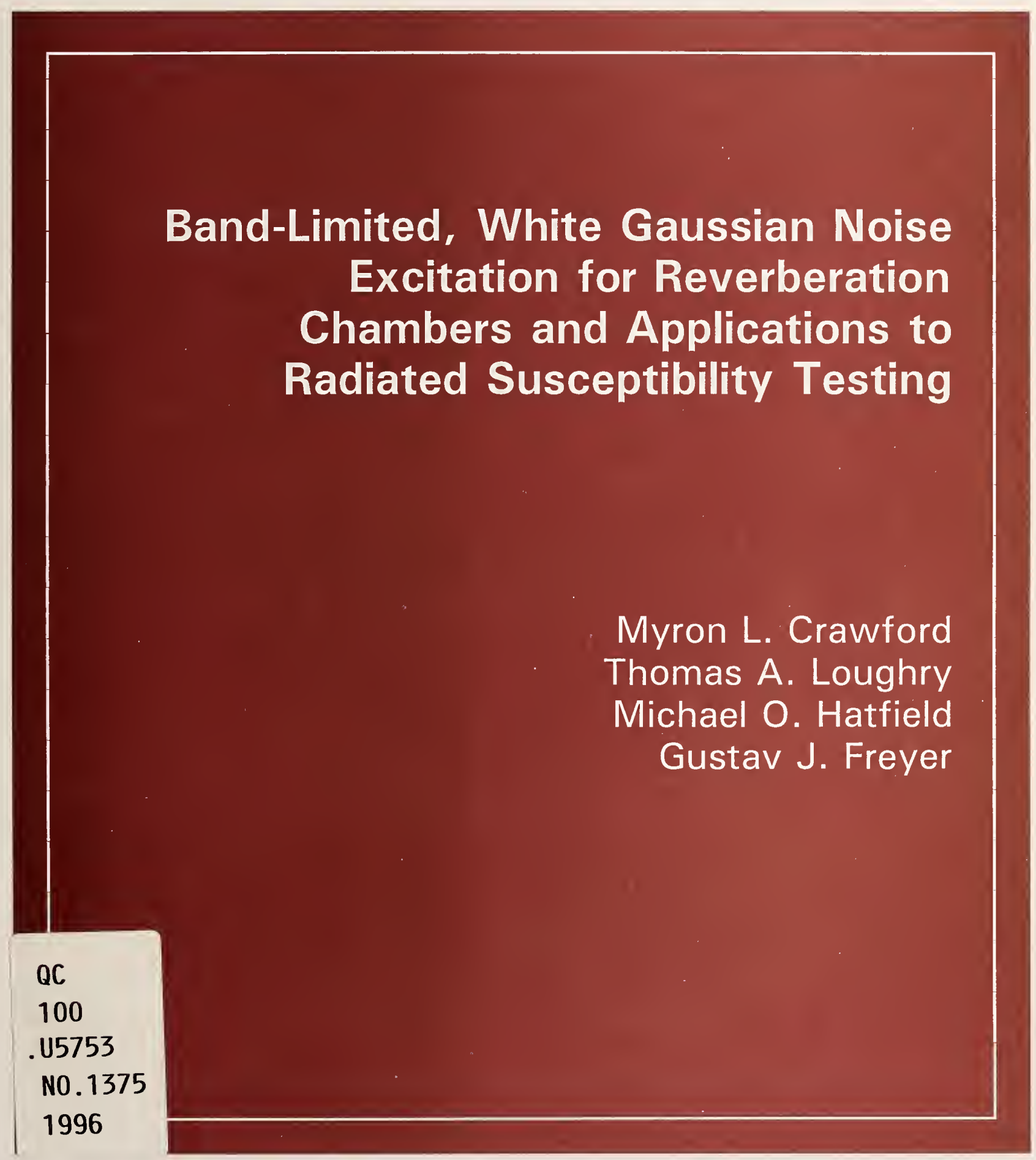





\section{Band-Limited, White Gaussian Noise Excitation for Reverberation Chambers and Applications to Radiated Susceptibility Testing}

Myron L. Crawford

Thomas A. Loughry

Michael O. Hatfield

Gustav J. Freyer

Electromagnetic Fields Division

Electronics and Electrical Engineering Laboratory

National Institute of Standards and Technology

325 Broadway

Boulder, Colorado 80303-3328

January 1996

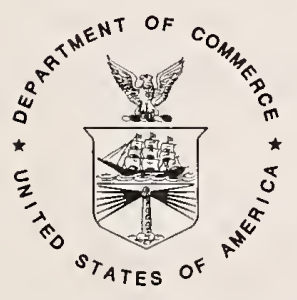

U.S. DEPARTMENT OF COMMERCE, Ronald H. Brown, Secretary TECHNOLOGY ADMINISTRATION, Mary L. Good, Under Secretary for Technology NATIONAL INSTITUTE OF STANDARDS AND TECHNOLOGY, Arati Prabhakar, Director 
National Institute of Standards and Technology Technical Note Natl. Inst. Stand. Technol., Tech. Note 1375, 116 pages (January 1996) CODEN:NTNOEF

\section{U.S. GOVERNMENT PRINTING OFFICE \\ WASHINGTON: 1996}

For sale by the Superintendent of Documents, U.S. Government Printing Office, Washington, DC 20402-9325 


\section{FOREWORD}

The research described in this technical note represents the results of a demonstration of the characteristics of the electromagnetic environment (EME) produced in a reverberation chamber by Band-Limited, White Gaussian Noise (BLWGN) excitation followed by a comprehensive test program. The three-phase test program was specifically planned to address the feasibility of using the BLWGN technique for conducting system susceptibility and shielding effectiveness tests.

The primary objectives of the research were to:

- characterize the EME produced by exciting a reverberation chamber with BLWGN,

- $\quad$ evaluate sensor and probe performance with BLWGN excitation, and

- demonstrate the feasibility of using BLWGN for radiated susceptibility and shielding effectiveness testing.

The tests were conducted at the reverberation and anechoic chamber facilities of the National Institute of Standards and Technology (NIST) and the reverberation chamber of the Naval Surface Warfare Center, Dahlgren Division (NSWCDD). The tests used much of the hardware and software developed by the USAF Phillips Laboratory for implementing the BLWGN excitation technique.

The reverberation chambers were excited both conventionally with $\mathrm{CW}$ and swept frequency signals and mechanical stirring, and with the BLWGN technique. The BLWGN excitation included noise bandwidths of $2,4,10,20$, and $50 \mathrm{MHz}$. The frequency range covered was $200 \mathrm{MHz}$ to 18 GHz. The NIST reverberation chamber typically is not used below $200 \mathrm{MHz}$ as a mode-stirred chamber because of the falloff in mode density below this frequency. Limited data were obtained, however, as low as $50 \mathrm{MHz}$ where only a few modes exist in the NIST chamber.

A variety of sensors and probes were used for chamber EME characterization as well as for probe evaluations. These included calibrated log periodic and dual ridged horn antennas, an $8 \mathrm{~mm}$ dipole antenna, surface mounted D-Dot and B-Dot probes, and a free field B-Dot probe.

The response of several test articles including standard TEM cells, a special common test article (CTA) for electromagnetic vulnerability tests, an instrumented $7.0 \mathrm{~cm}(2.75 \mathrm{in})$ folding-fin aircraft rocket, and a panel for shielding effectiveness measurements were evaluated to satisfy the test objectives.

The test team consisted of Captain Thomas A. Loughry, SRA David Little, and SRA Chris VanZandt of the USAF Phillips Laboratory; Michael O. Hatfield of the Naval Surface Warfare Center, Dahlgren Division; Myron L. Crawford and William Riddle of the National Institute of Standards and Technology; Gustav J. Freyer of Universal Systems Incorporated; and Tony Keyes of EG\&G. 



\section{CONTENTS}

FOREWORD $\ldots \ldots \ldots \ldots \ldots \ldots \ldots \ldots \ldots \ldots \ldots \ldots \ldots \ldots \ldots$ iii

LIST OF TABLES $\ldots \ldots \ldots \ldots \ldots \ldots \ldots \ldots \ldots \ldots \ldots \ldots \ldots \ldots \ldots \ldots$ vi

LIST OF FIGURES $\ldots \ldots \ldots \ldots \ldots \ldots \ldots \ldots \ldots \ldots \ldots \ldots \ldots \ldots \ldots \ldots$

1. INTRODUCTION AND BACKGROUND $\ldots \ldots \ldots \ldots \ldots \ldots \ldots \ldots \ldots$

2. BAND-LIMITED, WHITE GAUSSIAN NOISE EXCITATION . . . . . . . . . 3

2.1 Mode Mixing Through Frequency Agility $\ldots \ldots \ldots \ldots \ldots \ldots \ldots$

2.2 Implementing BLWGN Excitation $\ldots \ldots \ldots \ldots \ldots \ldots \ldots \ldots \ldots \ldots$

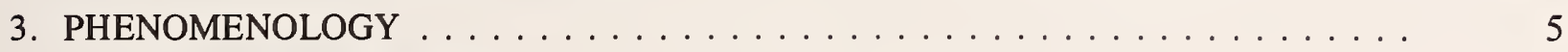

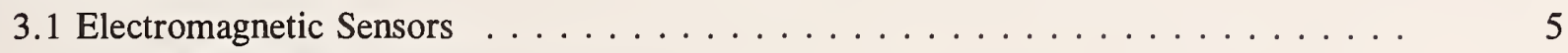

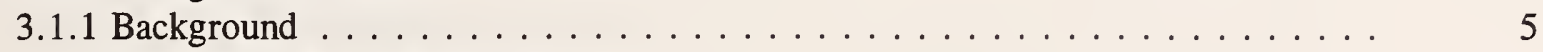

3.1.2 Theoretical Transfer Functions $\ldots \ldots \ldots \ldots \ldots \ldots \ldots \ldots \ldots \ldots$

3.1.3 Inherent Sensor Uncertainties . . . . . . . . . . . . . . . . . 6

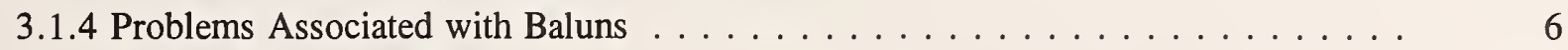

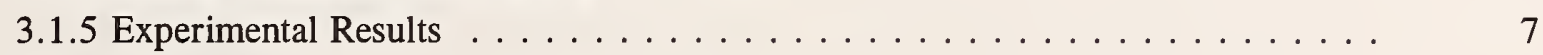

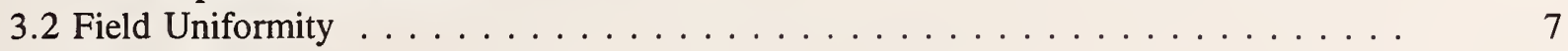

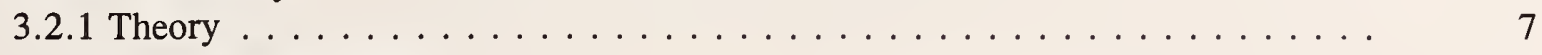

3.2.2 Experimental Results $\ldots \ldots \ldots \ldots \ldots \ldots \ldots \ldots \ldots \ldots \ldots \ldots \ldots$

3.3 Peak-to-Average Ratio . . . . . . . . . . . . . . . . . . . . 9

3.4 Power Density Enhancement Using Multiple Sources . . . . . . . . . . . . . . . 11

3.5 Time to Achieve Statistical Averaging . . . . . . . . . . . . . . . . . 12

4. EQUIPMENT UNDER TEST RESPONSES . . . . . . . . . . . . . . . 13

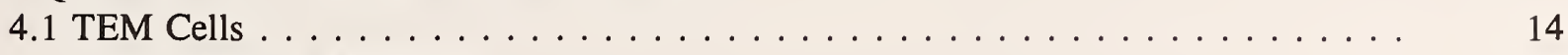

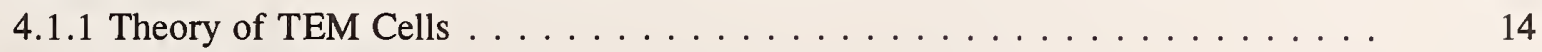

4.1.2 Experimental Results . . . . . . . . . . . . . . . . . . . . 15

4.1.3 Explanation of Results and Coupling Cross Sections . . . . . . . . . . . . 15

4.2 Electromagnetic Vulnerability Testing . . . . . . . . . . . . . . . . . . 17

4.2.1 Test Item Description . . . . . . . . . . . . . . . . . . . . . . 17

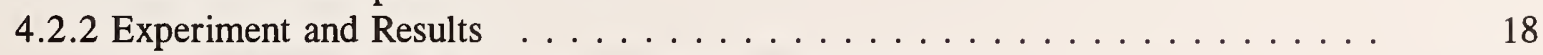

4.2.2.1 Band-Limited, White Gaussian Noise Excitation . . . . . . . . . . . . 19

4.2.2.2 Continuous Wave Excitation Using Mechanical Stirring . . . . . . . 20

4.2.2.3 Comparison of Noise Data to Continuous Wave Data . . . . . . . . 20

4.2.2.4 Comparison of Chamber Input Power Required for Upset . . . . . . . 21

4.3 Hazards of Electromagnetic Radiation to Ordnance Testing . . . . . . . . . . . 21

4.3.1 Test Item Description . . . . . . . . . . . . . . . . . . . 21

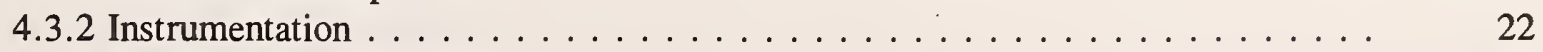

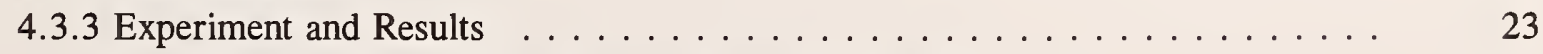

4.3.3.1 Band-Limited, White Gaussian Noise Excitation . . . . . . . . . . 23

4.3.3.2 Continuous Wave Excitation Using Mechanical Tuning . . . . . . . . 23

4.3.3.3 Comparison of Noise Data to Continuous Wave Data . . . . . . . . . 24

4.3.3.4 Anechoic Chamber Data . . . . . . . . . . . . . . . . 24

4.3.3.5 Comparison to Anechoic Chamber Data . . . . . . . . . . 25 


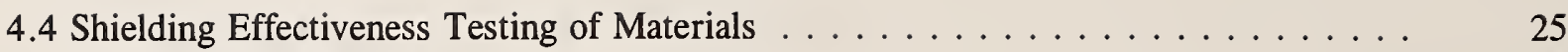

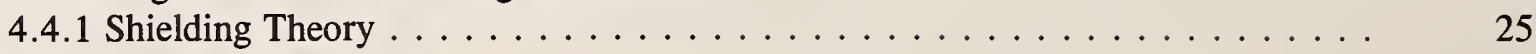

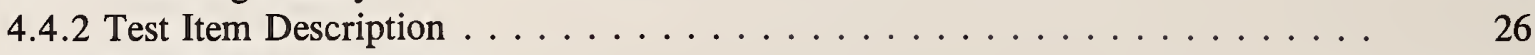

4.4 .3 Experiment and Results $\ldots \ldots \ldots \ldots \ldots \ldots \ldots \ldots \ldots \ldots$

4.4.3.1 Band-Limited, White Gaussian Noise Excitation . . . . . . . . . . . 26

4.4.3.2 Continuous Wave Excitation Using Mechanical Tuning . . . . . . . . . . 27

4.4.3.3 Comparison of BLWGN Data to Continuous Wave Data . . . . . . . . . 28

5. SPECIAL CONSIDERATIONS ASSOCIATED WITH TESTING WITH

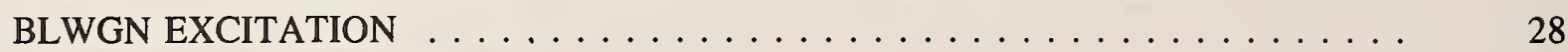

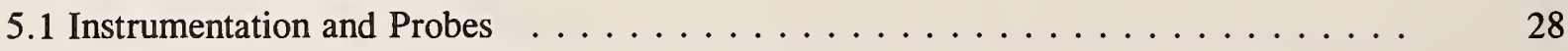

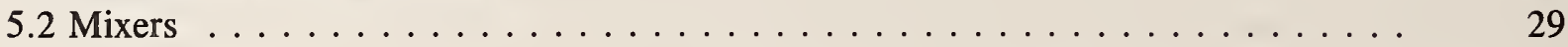

6. CONCLUSIONS ......................... 30

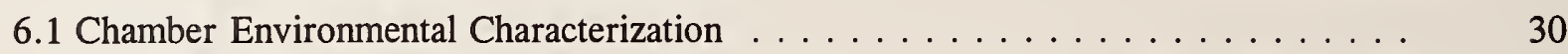

6.1 .1 Effective Mode Density $\ldots \ldots \ldots \ldots \ldots \ldots \ldots \ldots \ldots \ldots \ldots$

6.1 .2 Field Uniformity . . . . . . . . . . . . . . . . . 30

6.1.3 Field Peak-to-Average Ratio $\ldots \ldots \ldots \ldots \ldots \ldots \ldots \ldots \ldots . \ldots \ldots$

6.1.4 Time Between Peak Field Excitation . . . . . . . . . . . . . . . 31

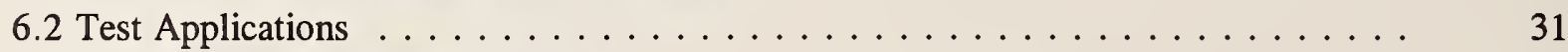

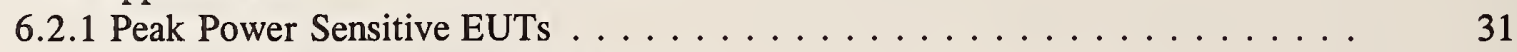

6.2 .2 Average Power Sensitive EUTs . . . . . . . . . . . . . . . 31

6.2.3 Shielding Effectiveness Measurements $\ldots \ldots \ldots \ldots \ldots \ldots \ldots \ldots . \ldots \ldots$

6.3 Test Considerations . . . . . . . . . . . . . . . . . . . 31

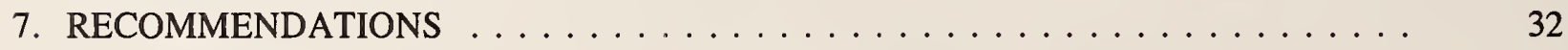

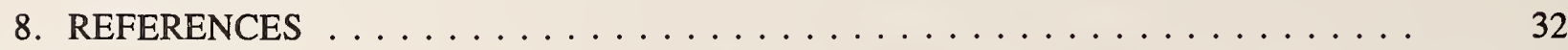


Table

\section{LIST OF TABLES}

3.1 Standard deviation of field ratios for different number of effective modes. . . . . . . 8

4.1 Mode cutoff and resonant frequency analysis of $12 \mathrm{~cm} \times 12 \mathrm{~cm}$ TEM cell. . . . . . 18

4.2 Mode cutoff and resonant frequency analysis of $3 \mathrm{~cm} \times 6 \mathrm{~cm}$ TEM cell. . . . . . 18

4.3 Frequency ranges for swept BLWGN excitation testing of folding-fin aircraft rocket.

\section{LIST OF FIGURES}

$\underline{\text { Figure }}$

$\underline{\text { Page }}$

2-1. Generating and measuring NBWGN excited fields. . . . . . . . . . . . 35

2-2. Theoretical spectrum generated by NBWGN method. . . . . . . . . . . . 35

2-3. Actual output of NBWGN excitation method with excitation method with a center frequency of $1.5 \mathrm{GHz}$ and four agility bandwidths. . . . . . . . .

3-1. Probe-balun configuration. $\ldots \ldots \ldots \ldots \ldots \ldots \ldots \ldots$

3-2. Field uniformity with $10 \mathrm{MHz}$ of NBWGN over 1 to $2 \mathrm{GHz} \ldots \ldots \ldots$

3-3. Field uniformity Relative Frequency Distribution for $2 \mathrm{MHz}$ of NBWGN

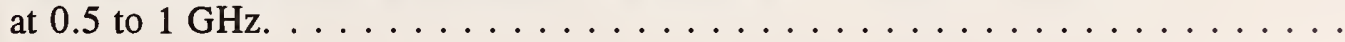

3-4. Field uniformity Relative Frequency Distribution for $10 \mathrm{MHz}$ of NBWGN

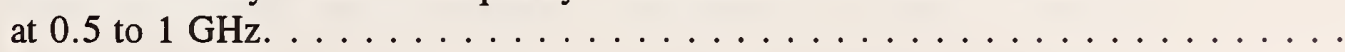

3-5. Field uniformity Relative Frequency Distribution for $20 \mathrm{MHz}$ of NBWGN

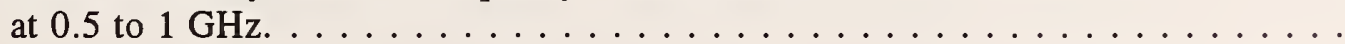

3-6. Field uniformity Relative Frequency Distribution for $50 \mathrm{MHz}$ of NBWGN

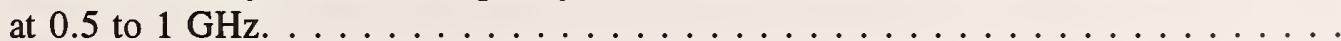

3-7. Field uniformity Relative Frequency Distribution for $2 \mathrm{MHz}$ of NBWGN

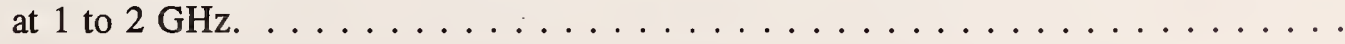

3-8. Field uniformity Relative Frequency Distribution for $10 \mathrm{MHz}$ of NBWGN at 1 to $2 \mathrm{GHz}$.

3-9. Field uniformity Relative Frequency Distribution for $20 \mathrm{MHz}$ of NBWGN at 1 to $2 \mathrm{GHz}$. 
3-10. Field uniformity Relative Frequency Distribution for $50 \mathrm{MHz}$ of NBWGN

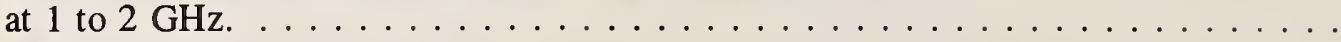

3-11. Field uniformity Relative Frequency Distribution for $2 \mathrm{MHz}$ of NBWGN at 2 to $4 \mathrm{GHz}$.

3-12. Field uniformity Relative Frequency Distribution for $10 \mathrm{MHz}$ of NBWGN at 2 to $4 \mathrm{GHz}$.

3-13. Field uniformity Relative Frequency Distribution for $20 \mathrm{MHz}$ of NBWGN at 2 to $4 \mathrm{GHz}$.

3-14. Field uniformity Relative Frequency Distribution for $50 \mathrm{MHz}$ of NBWGN at 2 to $4 \mathrm{GHz}$.

3-15. Field uniformity Relative Frequency Distribution for $2 \mathrm{MHz}$ of NBWGN at 4 to $8 \mathrm{GHz}$.

3-16. Field uniformity Relative Frequency Distribution for $10 \mathrm{MHz}$ of NBWGN

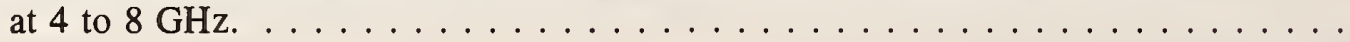

3-17. Field uniformity Relative Frequency Distribution for $20 \mathrm{MHz}$ of NBWGN

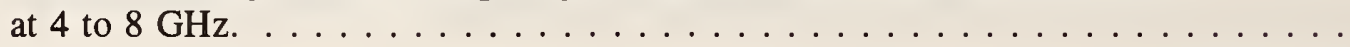

3-18. Field uniformity Relative Frequency Distribution for $50 \mathrm{MHz}$ of NBWGN

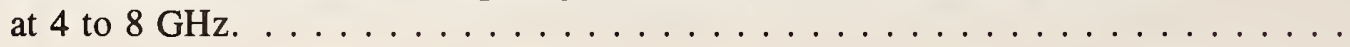

3-19. Field uniformity Relative Frequency Distribution for $2 \mathrm{MHz}$ of NBWGN

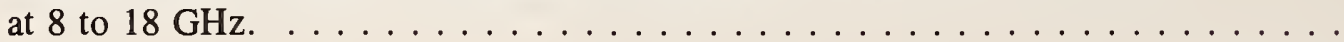

3-20. Field uniformity Relative Frequency Distribution for $10 \mathrm{MHz}$ of NBWGN at 8 to $18 \mathrm{GHz}$.

3-21. Field uniformity Relative Frequency Distribution for $20 \mathrm{MHz}$ of NBWGN

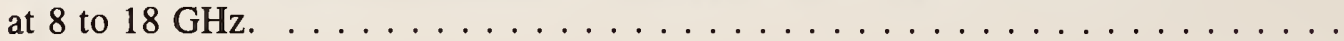

3-22. Field uniformity Relative Frequency Distribution for $50 \mathrm{MHz}$ of NBWGN

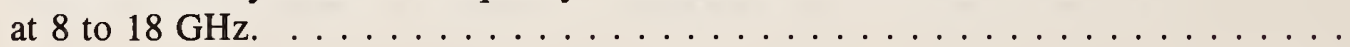

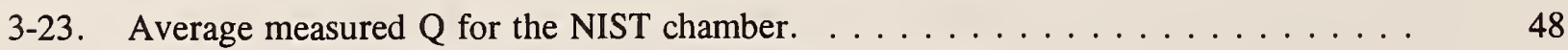

3-24. Equivalent modes per megahertz of NBWGN available for mode mixing. . . . . . 48

3-25. Chamber power density peak-to-average ratio measurement for NBWGN excitation. 
3-26. Chamber power density peak-to-average ratio measurement for $\mathrm{CW}$ (mechanical tuning) and $10 \mathrm{MHz}$ BW NBWGN excitation as a function

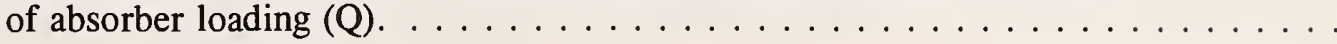

3-27. Block diagram of system for increasing test field inside reverberation chamber using a single noise source and mixer and two rf amplifiers.

3-28. Block diagram of system for increasing test field inside reverberation chamber using two noise sources and mixers and two rf amplifiers. .

3-29. Power density measured inside NIST reverberation chamber excited using single noise source, (a) power density from single and dual inputs, (b) increase in power density from single input to dual input ( $\mathrm{Pd}$ dual $-\mathrm{Pd}$ single).......

3-30. Power density measured inside NIST reverberation chamber excited using two noise sources, (a) Power density from single and dual inputs, (b) Increase in power density from single input to dual input. . . . . . . . . . . . .

3-31. Block diagram of system for measuring transient response and field uniformity of NBWGN excited chamber. . . . . . . . . . . . . . . . . . .

3-32. BLWGN $10 \mathrm{MHz}$ BW time domain data for NIST reverberation chamber at $1 \mathrm{GHz}$.

3-33. BLWGN $50 \mathrm{MHz}$ BW time domain data for NIST reverberation chamber at $1 \mathrm{GHz}$.

4-1. Geometry for an apertured TEM cell. . . . . . . . . . . . . . .

4-2. SE measurements of NIST $12 \mathrm{~cm}$ TEM cell obtained using NIST reverberating

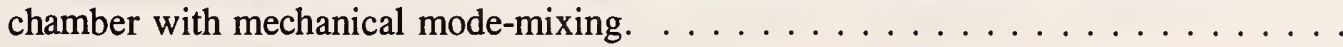

4-3. SE measurements of NIST $6 \mathrm{~cm}$ TEM cell obtained using NIST reverberating chamber with mechanical mode-mixing. . . . . . . . . . . . . .

4-4. Effect of cross-section bandwidth smaller than bandwidth of gating function. . . . .

4-5. Effect of cross-section bandwidth greater than bandwidth of gating function. . . . . .

4-6. Narrow cross-section resonance of $12 \mathrm{~cm}$ TEM cell measured using BLWGN. . . .

4-7. Wide cross-section resonance of $6 \mathrm{~cm}$ TEM cell measured with BLWGN. . . . . . .

4-8. Common test article showing interconnections and functions. . . . . . . . . .

4-9. Susceptibility profile of common test article obtained using CW excitation in the NSWCDD mechanically mode-mixed reverberation chamber. . . . . . . . . . 
4-10. Set-up for performing susceptibility tests of common test article in a reverberation chamber.

4-11. Block diagram of system for performing susceptibility tests of common test article in NIST reverberation chamber using BLWGN excitation. . . . . . . . . . .

4-12. Block diagram of system for performing susceptibility tests of common test article in NIST reverberation chamber using CW excitation with mechanical mode-mixing.

4-13. Susceptibility profile of common test article obtained with BLWGN (5 bandwidths). . . . . . . . . . . . . . . . . . .

4-14. Peak and average power susceptibility levels of common test article obtained with $\mathrm{CW}$ excitation and mechanical mode mixing.

4-15. Difference between peak and average power susceptibility levels of common test article. . . . . . . . . . . . . . . . . . . . .

4-16. Comparison of BLWGN and average CW (mechanical mode mixing) power densities data for common test article upset.

4-17. Difference between CW (mechanical mode mixing) average and BLWGN data for common test article upset. . . . . . . . . . . . . . . . .

4-18. Difference between CW (mechanical mode mixing) peak and BLWGN data for common test article upset.

4-19. Difference between input power to NIST reverberation chamber required for upset of common test article determined from average of all probe and antenna measurements and from each individual sensor.

4-20. Diagram of modified folding-fin aircraft rocket. $\ldots \ldots \ldots \ldots \ldots \ldots$

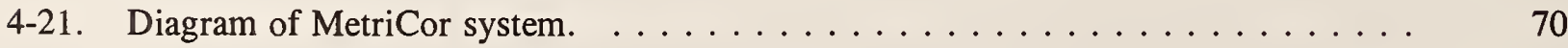

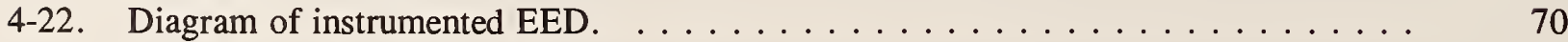

4-23. Folding-fin aircraft rocket mounted on styrofoam block in reverberation chamber.

4-24. Block diagram - BLWGN set-up for testing susceptibility of folding-fin aircraft rocket in NIST reverberation chamber. . . . . . . . . . . . .

4-25. Folding-fin aircraft rocket response -0.05 to $0.5 \mathrm{GHz}$ data. . . . . . . . . . . 73

4-26. Folding-fin aircraft rocket response -0.5 to $1 \mathrm{GHz}$ data. . . . . . . . . . . . . 74 
4-27. Folding-fin aircraft rocket response -1 to $2 \mathrm{GHz}$ data. . . . . . . . . . . 75

4-28. Folding-fin aircraft rocket response -2 to $4 \mathrm{GHz}$ data. . . . . . . . . . . . 76

4-29. Block diagram of CW, mechanical mode-mixing set-up for testing susceptibility of folding-fin aircraft rocket in NIST reverberation chamber.

4-30. Response of folding-fin aircraft rocket to $\mathrm{CW}$ excitation with

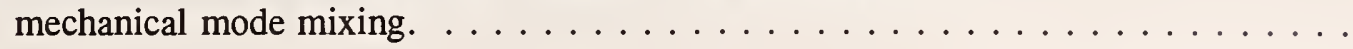

4-31. Average CW response of folding-fin aircraft rocket vs. noise response

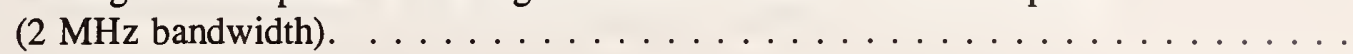

4-32. Folding-fin aircraft rocket in anechoic chamber.

4-33. Diagram of anechoic chamber discrete frequency set-up. . . . . . . . . . .

4-34. Folding-fin aircraft rocket response to vertically polarized illumination at $553.5 \mathrm{MHz}$ and $631 \mathrm{MHz} \ldots \ldots \ldots \ldots \ldots \ldots \ldots$

4-35. Folding-fin aircraft rocket response to horizontally polarized illumination

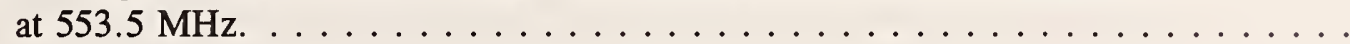

4-36. Folding-fin aircraft rocket response to horizontally polarized illumination

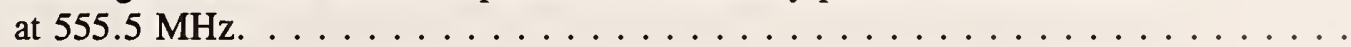

4-37. Folding-fin aircraft rocket response to horizontally polarized illumination

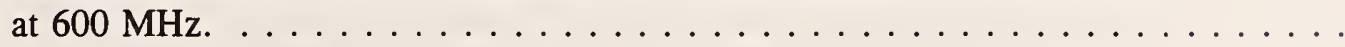

4-38. Folding-fin aircraft rocket response to horizontally polarized illumination

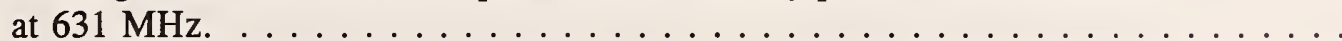

4-39. Diagram of anechoic chamber swept frequency set-up. . . . . . . . . . .

4-40. Anechoic chamber response of folding-fin aircraft rocket. . . . . . . . . . . . .

4-41. Folding-fin aircraft rocket anechoic chamber data vs. reverberation chamber $2 \mathrm{MHz}$ noise data.

4-42. Folding-fin aircraft rocket anechoic chamber data vs. reverberation chamber $2 \mathrm{MHz}$ noise data corrected for polarization factor.

4-43. "NESTED" chamber technique for collecting SE data. . . . . . . . . . . .

4-44. Photo of wire mesh window 
4-45. Photo of test fixture used for measuring the shielding effectiveness of shielded window using the nested reverberation chamber technique. . . . . . . . .

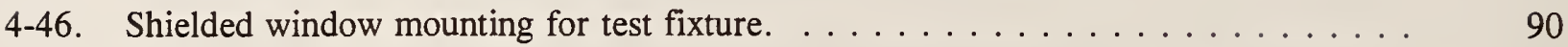

4-47. Window SE test fixture mounted inside NIST reverberation chamber. . . . . . . . . 91

4-48. Reverberation chamber $\mathrm{CW}$ (mechanical mode mixing) test set-up for SE measurements. . . . . . . . . . . . . . . . . . . .

4-49. Reverberation chamber BLWGN test set-up for SE measurements. . . . . . . . . . 93

4-50. Results of window SE measurements made using BLWGN technique

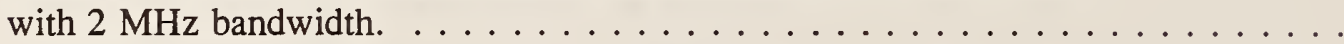

4-51. Results of window SE measurements made using BLWGN technique with $10 \mathrm{MHz}$ bandwidth. . . . . . . . . . . . . . . . .

4-52. Results of window SE measurements made using BLWGN technique

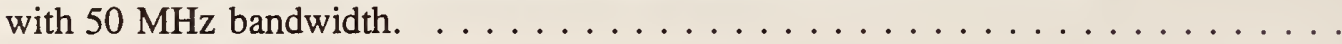

4-53. Comparison of baseline data collected from the test fixture and from the reverberation chamber. . . . . . . . . . . . . . . . . .

4-54. Results of window SE measurements made using BLWGN excitation with and without window mounted to the test fixture. . . . . . . . . . .

4-55. Results of window SE measurements made using CW (mechanical mode mixing) excitation with and without window mounted to the test fixture.

4-56. Difference in SE obtained using BLWGN and mechanical mode mixing. . . . . . . 97

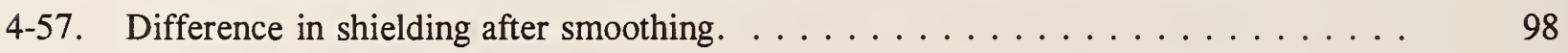

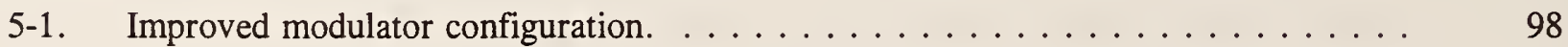




\title{
BAND-LIMITED, WHITE GAUSSIAN NOISE EXCITATION OF REVERBERATION CHAMBERS AND APPLICATIONS TO RADIATED SUSCEPTIBILITY TESTING
}

\author{
Myron L. Crawford \\ National Institute of Standards and Technology \\ Boulder, CO 80303 \\ Captain Thomas A. Loughry \\ USAF Phillips Laboratory \\ Kirtland AFB, NM 87117 \\ Michael O. Hatfield \\ Naval Surface Warfare Center, Dahlgren Division \\ Dahlgren, VA 22448 \\ Gustav J. Freyer \\ Universal Systems Incorporated \\ Monument, CO 80132
}

\begin{abstract}
This report gives the results of demonstration tests conducted to (1) evaluate the electromagnetic environment (EME) produced by band-limited, white gaussian noise (BLWGN) excitation of a reverberation chamber and to verify its applications to susceptibility and shielding effectiveness (SE) testing. Data were collected to compare the EME produced in a reverberation chamber by $\mathrm{CW}$ and swept frequency excitation using both mechanical stirring and BLWGN to excite the cavity mode structure. The feasibility of using the BLWGN technique for radiated susceptibility testing was evaluated by comparison with mechanical stirring in a reverberation chamber and with anechoic chamber results. Within normal measurement uncertainties, the response of both types of systems were the same for mechanical stirring and for BLWGN excitation and were consistent with the results obtained in the anechoic chamber.
\end{abstract}

Key words: electromagnetic environment; mode stirring; noise; quality factor; reverberation chamber; shielding effectiveness

\section{INTRODUCTION AND BACKGROUND}

Mode mixing a shielded enclosure to improve the electromagnetic compatibility (EMC) test results obtained using an enclosure was first proposed in 1968 [1]. Since then measurement procedures have been developed for implementing mechanical mode-mixing techniques for both radiated susceptibility and emission testing [2-6] with considerable success.

During the 1980s and early 1990s, agencies including the U.S. Air Force's Phillips Laboratory (PL) (and its predecessor the Air Force Weapons Laboratory), Kirtland AFB, NM; the National Institute of Standards and Technology (NIST), Boulder, CO; and the Naval Surface Warfare Center, Dahlgren Division (NSWCDD), Dahlgren, VA, were investigating various low cost methods of 
performing radio frequency precharacterization of systems identified for high power microwave testing. Specifically, techniques that would quickly and economically allow researchers to identify the frequencies and power densities at which a system would fail or upset were sought. It was hoped that EMC, EM vulnerability (EMV), and high power microwave vulnerability (HPMV) test costs could be reduced. During this same period, NIST and NSWCDD were developing the use of large reverberation chambers with mechanical tuners for reducing the costs of EMC/EMV testing.

The reverberation chamber technique offered several advantages over anechoic chamber or open range testing. These include: (1) the ability to generate high intensity radiated fields over large volumes using modest amounts of rf power, (2) the elimination of polarization and angle of incidence dependence, hence removal of physical rotation requirements of the equipment under test (EUT), (3) potential use of the test facility for both radiated susceptibility and emission testing with minor instrumentation changes, and (4) the resulting significant reduction in test time requirements.

These advantages are derived from the inherent characteristics of a reverberation chamber (simply a high quality factor ( $Q)$, metal enclosure) that acts as a multimoded resonant cavity with increased test fields. (The increase in the fields is due to the stored energy within the high Q cavity.) The chamber's tuner perturbs the fields inside the cavity, thus resulting in a statistically averaged isotropic impinging field at the EUT. One difficulty with this technique, however, is that dependence on the mechanical tuner requires that data be taken over many positions of the tuner to ensure that the EUT is exposed to the maximum field levels for the given test parameters. Further, a real-time homogeneous field within the chamber can only exist on the same time scale as the rotation rate of the tuner.

In an attempt to remove these limitations, the PL conducted an Independent Laboratory Initiated Research (ILIR) program to investigate the likelihood of mixing the modes within the chamber electronically. It was anticipated that this technique would provide real-time field uniformity within the chamber and eliminate dependence on the mechanical tuner. By November of 1991, the PL had demonstrated that electronic mode-mixing could be accomplished in a practical and economical manner by up-converting band-limited, white gaussian noise to microwave frequencies [7]. By using up-converted white gaussian noise to spread the energy over a narrow bandwidth, the technique randomly excites the reverberation chamber's resonant modes that lie within this bandwidth. This causes a spatial averaging out of the "hot" and "cold" spots that would typically exist in a mechanically tuned high-Q reverberation chamber at specific tuner positions. Since the bandwidth of the resulting signal is relatively narrow compared to the center frequency, the test results can still be considered characteristic of a discrete frequency. This technique is expected to reduce precharacterization test times by as much as 1000 over anechoic chamber or open range testing and by a factor of about 200 over mechanically mode-mixed reverberation chambers. Section 2 of this report contains a description of the band-limited, white gaussian noise (BLWGN) technique including some information for implementing this type of excitation in a reverberation chamber.

Demonstration tests were performed at NIST in January 1992 by the PL with representatives from the NSWCDD present. This then led to a fiscal year 1993 cooperative research project among the three organizations to investigate and validate the technique as an EMC/EMV testing tool. The research was conducted at NIST, Boulder, CO.

The project had two experimental phases. The first phase investigated the response characteristics of probes and instrumentation with noise excitation versus mechanical mode mixing in the NIST reverberation chamber. The results of these measurements are shown in section 3.1 of this report. 
The purpose of these measurements was to quantify and gain understanding of the electronically mode-mixed electromagnetic fields environment (EME) inside the reverberation chamber using theoretically characterizable devices. These results are needed to appropriately interpret results obtained with electronic mode-mixed fields in relation to more conventional field parameters. A second goal of phase I was to measure the field uniformity within the chamber as a function of frequency and modulation or agility bandwidth. These data were then compared with the mechanically mode mixed chamber data. The results of these measurements are contained in sections 3.2 and 3.3 of this report. The effects of chamber $Q$ were also investigated for the two methods of mode mixing. The final objective of phase I was to characterize some calibrated, apertured, TEM cells as a function of frequency and compare the results to the theoretically calculated cell resonant modes. These results are contained in section 4.1 of this report.

Phase II was an in depth susceptibility evaluation of three test articles again using both mechanical and electronic mode-mixing techniques. The results of these measurements are contained in sections $4.2,4.3$, and 4.4 of this report. The three test articles are unclassified and therefore lend themselves well to unrestricted reporting of research results. The test articles were provided by the NSWCDD. The first is a generic common test article (CTA). The CTA consists of two separate units that communicate with each other over a series of cables. The first unit generates a clock signal which is fed to the other unit, which then generates two separate binary counts for comparison. If the first unit detects any error in the counts, it reports the discrepancy by optical fiber link to the operator. A more detailed description of the CTA can be found in section 4.2.1. The CTA has been extensively characterized in both anechoic and reverberation chambers. The second test article is the Hazards of Electromagnetic Radiation to Ordnance (HERO) rocket. The HERO rocket is a $7.0 \mathrm{~cm}$ diameter, folding-fin aircraft rocket with a fiber optically instrumented electro-explosive device (EED). The last test article is a wire mesh shielded window used as a standard for comparing shielding effectiveness (SE) measurements. Like the CTA, the latter two test articles have been extensively characterized.

Conclusions resulting from this study and recommendation for future work are contained in sections 6 and 7 of this report.

\section{BAND-LIMITED, WHITE GAUSSIAN NOISE EXCITATION 2.1 Mode Mixing Through Frequency Agility}

The conventional method of mode-mixing uses a metallic paddle wheel to continuously or incrementally change the boundary conditions within the reverberation chamber while maintaining a constant excitation frequency. Field homogeneity is achieved by averaging the contribution of many different eigenfunctions to a given point in the chamber. An equivalent result can be obtained by maintaining the boundary conditions constant while the frequency is allowed to vary over a narrow interval about some center frequency. Then the contribution of each eigenfunction to a given spatial point in the chamber, in that narrow band of frequency, is averaged to provide the field homogeneity. The advantage of this method over mechanical mode mixing is that the eigenfunctions corresponding to that band can be stimulated simultaneously and randomly by spreading the power over a band of frequencies. Thus, because the energy stored in the fields in an arbitrarily small volume centered at any given spatial point is the sum of all the energies in the individual eigenmodes, field homogeneity is achieved in real time. Real-time homogeneity can drastically reduce test times. Even if individual eigenfunctions are changed by adding a test object or moving an existing test object within the 
chamber, the mean over the frequency band will remain constant (within the limits of the sampling variance).

Several techniques might be considered to provide the source frequency agility required. At minimum, however, the method used should meet the following criteria:

a. The power spectrum should be flat across the agility bandwidth to simplify data interpretation.

b. The signal should be ergodic (or at least loosely time stationary over the maximum averaging period of the test article and/or the power measuring instrument), so that real-time uniformity can be achieved.

c. The center frequency and bandwidth agility of the source should be variable over a wide parameter set to facilitate test flexibility.

d. The average output power should be continuously variable or variable in $3 \mathrm{~dB}$ or less increments, from $1 \mathrm{~W}$ to a couple of hundred watts to allow for a wide range of cavity quality factors and test article upset levels.

\subsection{Implementing BLWGN Excitation}

As shown in figure 2-1, BLWGN excitation uses band-limited, white gaussian noise (WGN) upconverted by performing double-sideband, suppressed-carrier modulation with an rf signal from a synthesized sweeper. The output is then amplified with a traveling wave tube (TWT) amplifier to meet the higher power requirements. Center frequencies between 0.1 and $50 \mathrm{GHz}$ and frequency agility bandwidths of less than one to as high as several hundred megahertz can be easily realized. In addition, the power output from the sweeper can be varied in $1 \mathrm{~dB}$ increments from $-120 \mathrm{dBm}$ to approximately $+15 \mathrm{dBm}$. Therefore, for practical purposes, the dynamic range of the technique is identically the dynamic range of the amplifier. Figure 2-2 shows the theoretical spectrum of the signal at each stage of the signal processing. The synthesized sweeper is used to generate the monochromatic signal which determines the center frequency of the output while the WGN source and low pass filters provide the frequency agility. WGN will maintain its statistical properties after having passed through a linear, invariant system such as a low pass filter [8].

In the set-up of figure $2-1$, the instrumentation is controlled by a central computer and data plotting is available from a standard plotter. The probes in the chamber provide a method of monitoring field strength. The instrumentation used to measure the power delivered by the probes must be capable of averaging over the agility bandwidth of the BLWGN. Scalar analyzers or standard power meters can be used; however, narrow band instruments such as spectrum analyzers and vector analyzers would require additional data reduction.

Figure 2-3 shows the actual output of the BLWGN at a fixed center frequency of $1.5 \mathrm{GHz}$, immediately after up-conversion as measured by a spectrum analyzer. The spectrum analyzer is used here to demonstrate the spreading of the energy across the agility bandwidth but should not be used as a measuring device during actual EMC testing. In practice, the scalar analyzer integrates over the spectrum shown in figure $2-3$ to give a single power measurement at the center frequency.

The configuration shown in figure 2-1 allows three different test modes to be accomplished. First, input power supplied to the chamber can be held constant while the frequency is swept at a 
predetermined rate. This is particularly useful when measuring electromagnetic shielding as a function of frequency. Second, the frequency can be held constant while the power into the chamber is incrementally increased. This has its strongest application in determining a system's susceptibility at a given frequency. The system of interest is placed in the chamber and is operated as the power density in the chamber is slowly increased until the system malfunctions. At this point, the power density in the chamber is measured using one of the probes to determine the upset threshold. Finally, both power and frequency can be held constant. This would typically be used for performing diagnostics on the chamber or providing long term exposure fields.

\section{PHENOMENOLOGY}

\subsection{Electromagnetic Sensors}

\subsubsection{Background}

The accurate measurement of microwave frequency electromagnetic fields is fundamental to the broadband electromagnetic characterization of any system. Measurements of continuous-wave coupling, shielding effectiveness, and narrow band electromagnetic vulnerability all depend directly on field measurements. It is common practice when conducting electromagnetic measurements in the reverberation chamber to use standard antennas such as log periodic or horn antennas to monitor fields. Because these antennas have large effective apertures at low frequencies (less than a few hundred megahertz), they can significantly load the chamber and reduce overall chamber Q [7]. Such antenna also tend to be bulky and limit overall usable chamber volume. These limitations can be overcome with the use of simple sensors such as electrically short dipole probes or the B-dot and Ddot probes. The B-dot consists of a small cylindrical loop antenna while the D-dot is composed of a small asymptotic dipole above a ground plane. They offer much smaller volumes as well as relatively smaller effective apertures. In fact, surface mount probes can be used in the chamber thus, requiring much less use of the test volume.

\subsubsection{Theoretical Transfer Functions}

Theoretical sensor transfer functions are often used when measuring narrow band electromagnetic fields at microwave frequencies. These functions convert sensor outputs to incident field values. Standard sensors such as the B-dot probe, D-dot probe, and antennas like standard gain horns are well understood [9-11]. Small dipole probes which use high impedance transmission lines typically are precalibrated in TEM cells or anechoic chambers to provide their transfer function.

When properly polarized to a free-space, planar incident wave, the transfer function relates probe output power to incident field power density. It can be expressed as follows for the B-dot and D-dot sensors or for a horn antenna:

$$
\frac{P_{m}}{P_{d_{n}}}=\frac{\mu_{o} A_{e}^{2}}{50 c} \omega^{2},
$$

B-dot: 
D-dot:

$$
\frac{P_{m}}{P_{d_{n}}}=\frac{50 \epsilon_{o} A_{e}^{2}}{c} \omega^{2},
$$

Horn:

$$
\frac{P_{m}}{P_{d_{n}}}=\frac{\pi c^{2} G}{\omega^{2}}
$$

where and $P_{m}$ is the power delivered to a $50 \Omega$ instrument by the sensor, $P_{d n}$ is the incident power density, $A_{e}$ is the sensor's equivalent area, $\mu_{0}$ is the permeability of free space, $\epsilon_{0}$ is the permittivity of free space, $c$ is the speed of light in a vacuum, $G$ is a horn's gain, and $\omega$ is the narrow band center radian frequency, respectively. It should be noted that these equations only apply well below the cutoff frequency of the B-dot and D-dot and within the design bandwidth of the horn antenna. When one uses these probes and antennas in a reverberation environment, a polarization factor of 2 must be accounted for [12] and their directivity simply becomes 1 .

\subsubsection{Inherent Sensor Uncertainties}

Measurement uncertainties can be attributed primarily to two sources, those errors introduced by the power measuring instrumentation and those introduced by the sensor itself. Power measurement errors appear both in the actual power measurement and system component calibrations, but these uncertainties can usually be controlled to within $\pm 1 \mathrm{~dB}$. Sensor uncertainties include opposing field or sensor leg electric field pickup, deviation from the ideal equations presented earlier, and slight uncertainties in the mechanical dimensions that determine equivalent area and gain. In practice, the first two sources of error dominate the last. The opposing field and leg pickup can be reduced by using multielement probes that provide a differential output for the desired field and a common mode output for extraneous pickup [12]. Thus, by using a $180^{\circ}$ summation device such as a hybrid coupler or a balun, common mode errors are reduced to within the limitations of the summation component. As will be shown shortly, a summation component such as a balun can introduce more error than it alleviates and therefore as not used in these experiments. Finally, the other significant source of error appears when the sensors are used near their cutoff frequencies. The B-dot probe, for example, introduces two poles in the transfer function due to the inductance and the transient time associated with the sensor loop [9]. The D-dot has a similar problem due to the asymptotic dipoles capacitance with the ground plane [10]. By definition, at cutoff, a $3 \mathrm{~dB}$ error will occur.

\subsubsection{Problems Associated with Baluns}

As previously mentioned, it is common practice to use a $180^{\circ}$ summation device to eliminate common mode problems associated with opposing field and sensor electric field leg pick up. One of the most popular summation devices is the balun, see figure 3-1. A seldom recognized problem with the balun, however, is errors created by leakage between the two input ports. A small amount of power leaks from one leg to the other and is transmitted back toward the sensor. A B-dot probe acts 
as a short while a D-dot probe acts like an open when viewing the Thevenin or Norton equivalent source impedance. Thus, power transmitted back towards the sensor will be reflected back again towards the balun. Reflections back from the sensor will add vectorially with the original signal according to their amplitude and phase and will create a ripple in the balun output as the frequency is swept across a wide bandwidth. As shown in figure 3-1, the source match of the probe can be improved by the introduction of in-line attenuators. Employing a geometric series identity, accounting for both legs of the probe, and using a small argument approximation for the logarithm allow the error associated with the balun leakage to be expressed as:

$$
P_{E R R}(d B) \propto \pm \frac{40}{\ln (10)} 10^{\left[\frac{-\left(B_{I L}+S_{R L}+2 A\right)}{20}\right]}
$$

where $B_{I L}, S_{R L}$, and $A$ are the balun leg-to-leg insertion loss, the sensor return loss, and the attenuator value, respectively, all in decibels. For example, if it is assumed that the sensor has a return loss of $0 \mathrm{~dB}$ (short or open) and no attenuators are used, a balun with a leakage insertion loss of greater than $15 \mathrm{~dB}$ must be used to achieve an uncertainty of less than $3 \mathrm{~dB}$. Many actual baluns cannot meet this specification. If a $10 \mathrm{~dB}$ attenuator is added to each leg, the same balun can achieve an error of less than $0.3 \mathrm{~dB}$; however, the sensor sensitivity is reduced by $10 \mathrm{~dB}$.

\subsubsection{Experimental Results}

Fields were generated in the chamber using discrete frequencies for both $\mathrm{CW}$ (mechanical mode mixing) and $2,10,20$, and $50 \mathrm{MHz}$ of BLWGN excitation. The fields were then measured using a surface mount D-dot, free field B-dot, surface mount B-dot, horn probe, NIST reference horn, and an $8 \mathrm{~mm}$ dipole with a resistive lead. The discrete frequencies measured included $0.5,0.6,0.8,1.0$, $1.5,2.0,3.04 .04 .5,5.0,5.5,6.0,6.5,7.5,8.0,10.0,12.0,14.0,16.0$, and $18.0 \mathrm{GHz}$. Each sensor was used in the appropriate frequency range matching its usable bandwidth. In all cases except for the $8 \mathrm{~mm}$ dipole with resistive lead, all sensors measured the fields accurately to within the field uniformity as described in section 3.2 . The $8 \mathrm{~mm}$ dipole with high resistance leads uses a diode detector and a low pass filter circuit which caused erroneous readings when used with the BLWGN excitation technique. The low pass filtering associated with the $8 \mathrm{~mm}$ dipole probe's high resistance lead and low pass filter attenuated the upper frequency spectrum of the down converted noise and thus tended to lower the probe's indicated field measurements as compared with the other sensors.

\subsection{Field Uniformity}

\subsubsection{Theory}

The purpose of mode mixing in a reverberation chamber is to provide a statistically averaged uniform or homogeneous field throughout the volume of the chamber. A measure of uniformity $\psi$ can be expressed as

where $\mathrm{w}_{\max }$ and $\mathrm{w}_{\min }$ are the maximum and minimum possible local average energy densities found anywhere in the chamber. Because power density and energy density are directly related, minimum

$$
\psi=10 \log \frac{w_{\max }}{w_{\min }},
$$


Table 3.1. Standard deviation of field ratios for different number of effective modes.

\begin{tabular}{rrrrrc}
\hline $\mathrm{N}$ & $\sigma$ & $\mathrm{N}$ & $\sigma$ & $\mathrm{N}$ & $\sigma$ \\
\hline 2 & 11.35 & 16 & 2.54 & 350 & 0.515 \\
3 & 8.47 & 18 & 2.35 & 600 & 0.380 \\
4 & 6.58 & 20 & 2.23 & 850 & 0.323 \\
5 & 5.61 & 25 & 2.03 & 1100 & 0.286 \\
6 & 4.72 & 29 & 1.84 & 1350 & 0.260 \\
7 & 4.49 & 35 & 1.65 & 1600 & 0.238 \\
8 & 3.88 & 45 & 1.46 & 1850 & 0.220 \\
10 & 3.47 & 57 & 1.26 & 2100 & 0.200 \\
12 & 3.15 & 82 & 1.02 & 2350 & 0.190 \\
14 & 2.84 & 100 & 0.934 & 2600 & 0.186 \\
\hline
\end{tabular}

and maximum average power density can replace average energy density in eq (3-5) with equal validity.

Predicting field uniformity for a given reverberation chamber has been previously treated [7]. Table 3.1 shows the correlation between the number $\mathrm{N}$ of modes and the standard deviation $\sigma$ of the ratio of two average power density measurements taken at two random positions. Traditionally, $\mathrm{N}$ has been calculated using the theoretical mode density expressed as

$$
N=\frac{8 \pi V}{c^{3}} f^{2} d f
$$

where $\mathrm{V}$ is the cavity volume, $\mathrm{c}$ is the speed of light, $\mathrm{f}$ is the center frequency, and $\mathrm{df}$ is the bandwidth over which the modes are stirred. This, however, does not account for the overlapping of modes due to their non zero bandwidth. This can be, at least heuristically, corrected for by taking the ratio of the noise bandwidth to the bandwidth $\mathrm{BW}_{\mathrm{Q}}$ of the resonant modes giving the resulting reduced number $\mathrm{N}_{\mathrm{Q}}$ of effective modes. The resonant mode bandwidth is calculated as

$$
B W_{Q}=\frac{f}{Q}
$$

If $\mathrm{N}_{\mathrm{Q}}$ is less than $\mathrm{N}$, then $\mathrm{N}_{\mathrm{Q}}$ should be used in place of $\mathrm{N}$ in table 3.1. As indicated in ref. [7], the actual uniformity as defined above can be related to the field standard deviation as 


\subsubsection{Experimental Results}

The uniformity of fields in the chamber can be measured by placing the same probe in two different locations and measuring the difference in field intensity as a function frequency. It is assumed that the statistics of the fields vary little over small changes in frequency. Thus, sampling the fields over a small frequency span at a single location in the chamber is equivalent to sampling several chamber locations at a single frequency. Typical data are shown in figure 3-2 for the 1 to $2 \mathrm{GHz}$ band using $10 \mathrm{MHz}$ of BLWGN. A more demonstrative way to represent the same data is to use a relative frequency distribution. The ordinate is broken down into 100 equal-sized cells of $\Delta \mathrm{dB}$ and the total number of data points falling within each cell is then plotted to form a relative frequency distribution (also referred to as a probability distribution). Figures 3-3 through 3-22 show the field uniformity relative frequency distribution for the 0.5 to 1,1 to 2,2 to 4,4 to 8 , and 8 to $18 \mathrm{GHz}$ for 2, 10, 20, and $50 \mathrm{MHz}$ of BLWGN.

The number of modes expected in the NIST chamber at these frequencies is very high. For example, at $1 \mathrm{GHz}$ and with $2 \mathrm{MHz}$ of BLWGN, the total modes available for stirring can be calculated from eq (3-6) as approximately 74. Equation (3-8) and table 3.1 show that the fields at these input parameters will not to vary more than $\pm 2.5 \mathrm{~dB}$. The data in figure 3-3 indicate much more variation in the average power density. However, the field uniformity stays relative the constant as the center frequency of the BLWGN is increased as indicated in figures 3-11 and 3-15. Equation (3-6) and table 3.1 suggest the opposite. This implies that some other mechanism may be at work to affect the actual number of modes available for mode mixing.

This effective lowering of the mode density may be explained by considering the finite $Q$ of the chamber as discussed above. The $\mathrm{Q}$ for the NIST chamber was measured using the methods described in refs. [7] and [13]. Figure 3-23 shows average results for $2,10,20$, and $50 \mathrm{MHz}$ of BLWGN. Of more interest, however, is the ratio of BLWGN bandwidth to $\mathrm{Q}$ bandwidth as described in eq (3-7). Figure 3-24 demonstrates this ratio $\mathrm{N}_{\mathrm{q}}$ per megahertz of BLWGN. For example, at 1 $\mathrm{GHz}, \mathrm{N}_{\mathrm{q}}$ is approximately 4 as read from figure 3-24. Hence, for $2 \mathrm{MHz}$ of BLWGN at $1 \mathrm{GHz}$, the equivalent modes available for mode mixing would be 8 . This corresponds to a uniformity of approximately $\pm 10 \mathrm{~dB}$ according to table 3.1 and eq (3-8). This is a much better predictor of the data in figure 3-23. The dropoff of uniformity with frequency is also explained by the dropoff of $N_{q}$ with frequency as shown in figure 3-24. Thus, the chambers $Q$ can and does have a profound effect on the ability of BLWGN (and mechanical mode mixing as well) to stir the fields in the chamber.

\subsection{Peak-to-Average Ratio}

The peak-to-average ratio of the fields in a mechanically stirred chamber is typically in the range 7 to $8 \mathrm{~dB}$ [6]. The same ratio can be demonstrated using BLWGN excitation. Figure 3-1 shows the fields measured using a B-dot probe and diode detector operating in its square law region and therefore the voltage shown on the vertical axis is directly proportional to power. Two traces are shown on the oscilloscope. The first is a real-time field variation, while the second is a ensemble average of many such traces. Hence, the peak-to-average ratio can be calculated by the ratio of the peak value of trace $1(6.3 \mathrm{mV})$ to the average value of trace $2(1.3 \mathrm{mV})$ or $6.8 \mathrm{~dB})$. 
The theoretical calculation of the peak-to-average ratio for the BLWGN excitation method follows the mechanical mode-mixing calculation [6] identically and hence accounts for experimental results indicating the same peak-to-average ratio in both cases. The mechanical mode-mixing calculation assumes that each position of the paddle wheel contributes a random independent sample of a mode structure defined as

$$
\begin{aligned}
& \phi^{2}(x, y, z)=\sin ^{2}\left(k_{x} x\right) \sin ^{2}\left(k_{y} y\right) \sin ^{2}\left(k_{z} z\right), \\
& k_{x}=\frac{m \pi}{a}, k_{y}=\frac{n \pi}{b}, k_{z}=\frac{p \pi}{d},
\end{aligned}
$$

where $\mathrm{m}, \mathrm{n}$, and $\mathrm{p}$ are integers, and $\mathrm{a}, \mathrm{b}$, and $\mathrm{d}$ are the dimensions of the chamber. The simplified eq (3-1) represents only single mode, and the multimode, vector nature of the field should be included in a future analysis. The paddle wheel directly perturbs the mode indices $k_{\mathrm{x}}, \mathrm{k}_{\mathrm{y}}$, and $\mathrm{k}_{\mathrm{z}}$ causing the sine arguments to behave like random variables. Here it is assumed that the amplitude of the mode does not change with the tuner position although in practice it will because of changing cavity input impedance mismatch. At this point, ref. [6] lets $\mathrm{x}, \mathrm{y}$, and $\mathrm{z}$ become the variable of integration and finds the expected value of $\phi^{2}(x, y, z)$. In reality, the probe being used to measure the fields is fixed at a point $\left(\mathrm{x}_{\mathrm{p}}, \mathrm{y}_{\mathrm{p}}, \mathrm{z}_{\mathrm{p}}\right)$ and the mode indices are the random variables. Relative to the math, it does not matter which is considered the random variable provided that the total normalized argument of the sine is a uniform random variable on the interval $-\pi$ to $+\pi$ or equivalently because the $\sin ^{2}$ repeats every $\pi$, the interval 0 to $\pi$. The average value of the fields at the point $\left(x_{p}, y_{p}, z_{p}\right)$ can be expressed equivalently as

$$
\bar{\phi}^{2}=E\left[\sin ^{2}\left(v_{1}\right) \sin ^{2}\left(v_{2}\right) \sin ^{2}\left(v_{3}\right)\right]
$$

where $\nu_{1}, \nu_{2}$, and $\nu_{3}$ are random variables with the following probability distributions

$$
\begin{array}{ll}
f\left(v_{1}\right)=\frac{1}{\pi} ; & 0 \leq v_{1} \leq \pi, \\
f\left(v_{2}\right)=\frac{1}{\pi} ; & 0 \leq v_{2} \leq \pi, \\
f\left(v_{3}\right)=\frac{1}{\pi} ; & 0 \leq v_{3} \leq \pi,
\end{array}
$$

Their joint distribution is

$$
f\left(v_{1}, v_{2}, v_{3}\right)=\frac{1}{\pi^{3}}
$$

and hence, 


$$
\bar{\phi}^{2}=\int_{0}^{\pi} \int_{0}^{\pi} \int_{0}^{\pi} \frac{1}{\pi^{3}} \sin ^{2}\left(v_{1}\right) \sin ^{2}\left(v_{2}\right) \sin ^{2}\left(v_{3}\right) d v_{1} d v_{2} d v_{3}=\frac{1}{8}
$$

which is equivalent to the ref. [6].

The frequencies $f$ at which resonant modes exist in the cavity are defined by

$$
f=\frac{c}{2 \pi \sqrt{\mu_{r} \epsilon_{r}}} \sqrt{k_{x}^{2}+k_{y}^{2}+k_{z}^{2}}
$$

Thus, if the frequency $\mathrm{f}$ is changed randomly over a small interval of frequency, as is the case in BLWGN excitation, $k_{x}, k_{y}$, and $k_{z}$ must change randomly but in accordance with eqs (3-15) and (316). Therefore, the same resulting peak-to-average value would be expected ( 8 to 1 , or roughly $9 \mathrm{~dB}$ for a single mode). In practice, the peak-to-average ratio rarely exceeds $8 \mathrm{~dB}$ for either mechanical or BLWGN mode mixing, although values as high as $9.8 \mathrm{~dB}$ have been observed in very large data samples.

As the $\mathrm{Q}$ of the chamber is reduced by loading it with rf absorber, the peak-to-average value in general decreases as shown in figure 3-26. As the $Q$ decreases, the bandwidth of each resonant mode increases. Eventually, several resonant modes overlap so the same excitation frequency will excite many modes instead of one. This has no effect on the average value but does tend to reduce the peak value. For example, if 10 modes are simultaneously stimulated, the maximum value can only be achieved if the peaks from each resonant mode are coincident at the same spatial location. The lower the $\mathrm{Q}$, the greater the number of overlapping modes and the less likely the maximums will coincide at the same locations. However, if the effectiveness of the mode mixing can be increased, for example, by increasing the noise bandwidth, then the typical peak-to-average ratios can be achieved even for low $Q$ cavities [3-14].

\subsection{Power Density Enhancement Using Multiple Sources}

An interesting feature of a reverberation chamber is the possibility of increasing the test fields inside the chamber by combining the output from rf sources. These sources may be operating at the same frequency or at different frequencies. If BLWGN stirring techniques are used, a single source can be used to drive multiple amplifiers with the same increase in test fields as if two incoherent sources were used. Block diagrams of the systems used to verify these possibilities are shown in figures 3-27 and 3-28. Figure 3-27 is for two coherent sources and figure 3-28 is for two incoherent sources. The results of the measurements are given in figures 3-29 and 3-30. Figures 3-29 and 3-30 show the power density, in $\mathrm{dBm}$, measured inside the chamber using $10 \mathrm{MHz}$ bandwidth filters with the BLWGN system for coherent and incoherent sources, respectively. Figures 3-3(b) and 3-4(b) show the difference between the power densities measured inside the chamber with a single source and with two sources, each having the same output as the single source, for the coherent and incoherent cases. (The (b) figures were obtained from the (a) data.) 
The above measurements were acquired from single locations of the transmitting and receiving antennas and reflect the effects of field uniformity. Based on field uniformity measurements, averaging of measurements from multiple locations should yield a flat $3 \mathrm{~dB}$ increase.

\subsection{Time to Achieve Statistical Averaging}

One of the major advantages of reverberation chamber testing is that an EUT is exposed to a statistically isotropic and randomized field. As a result of the isotropic illumination, the EUT need not be rotated to obtain all aspect angle susceptibility data.

However, the deterministic nature of the field as a function of tuner position for mechanical mode mixing could present a problem for testing some EUTs. For a fixed tuner position, the reverberation chamber EME will be characterized by a series of nodes and hot spots. These field variations are averaged and the field becomes statistically isotropic over a complete rotation of the tuner. However for some EUTs, particularly large EUTs, the response could be effected by the nonuniform illumination at a given tuner position. The effect is most pronounced for mode-tuned operations.

In the mode-tuned procedure, the tuner is stepped successively through a number of discrete positions. The tuner remains stationary at each step for an interval equal to or somewhat longer than the EUT response time. Thus, in the mode-tuned procedure any EUT response effects due to nonuniform illumination would be most apparent.

In the mode-stirred procedure, the tuner is rotated continuously. In most cases, the rotation rate is slow enough that the dwell time of a field configuration is equal to or greater than the EUT response time. Thus, for slow tuner rotation rates the EUT response should be the same as for the mode-tuned procedure.

To avoid the problem of a series of nonuniform configurations some testers use very high rotation rates in the mode-stirred procedure. The concept is to produce the field uniformity over a time interval which is short compared to the EUT response time. For example, if the EUT response time is $1 \mathrm{~s}$, then one might try to establish field uniformity on a time scale of 0.1 . This would require a rotation rate of $10 \mathrm{rev} / \mathrm{s}$. Some testers do, in fact, routinely operate at 1 to $5 \mathrm{rev} / \mathrm{s}$ [3-15]. However, the high rotation rates necessary to cover a wide range of EUT response times could pose operational problems for the chamber.

Since with BLWGN excitation the mode stirring is electronic, it seemed appropriate to investigate the time scales necessary to establish field uniformity. This investigation requires the measurement system to have a fast response time. For these measurements the scalar network analyzer was replaced with a fast digitizing signal analyzer. The test set-up is shown in figure 3-31. A typical data trace is shown in figure 3-32. The figure is a "snapshot" of the time dependence of the power density as well as a running average of power density integrated over the noise BW. The issue is how long an interval (the EME refresh time) is required before the noise excitation randomizes the chamber EME. The indication of randomization will be the recurrence of the expected peak power density as referenced to the average power density. Typical values of 7 to $8 \mathrm{~dB}$ are observed for mechanical mode-mixing in reverberation chambers. We will use a peak-to-average ratio of $7 \mathrm{~dB}$ as the refresh criterion.

To investigate the amplitude of the peak-to-average ratio for BLWGN and the average refresh time, data were collected for an ensemble of 4026 runs. Averaging provides better statistical data. 
However, the number of runs averaged, although a common value, was too large for proper evaluation of the refresh time. Since the data collected were the peak within a particular sampling time window, an event which exceeded any specified level (in this case, the $7 \mathrm{~dB}$ criterion) but which was less than the maximum value collected in that time window for any of the 4026 runs, would not be recorded. This saturation effect was demonstrated in one data set where all the measured values exceeded the $7 \mathrm{~dB}$ criterion. Therefore, these data can only define an upper bound on the refresh time.

Peak data at $1 \mathrm{GHz}$ for $10 \mathrm{MHz}$ noise $\mathrm{BW}$ are shown in figure 3-2. The average value of another 4026 runs completed under the same test conditions is also shown in the figure.

Data collection is initiated by the trigger signal at time zero. The settling time of about $2.5 \mu \mathrm{s}$ is apparent and is disregarded in the analysis.

The solid line in the figure is the $7 \mathrm{~dB}$ criterion for the peak power density. Using the data points which exceed the $7 \mathrm{~dB}$ reference line, the single sweep time, and the number of runs averaged, we can determine that the refresh time has an upper bound of less than $200 \mu \mathrm{s}$. A similar analysis of 50 $\mathrm{MHz} \mathrm{BW}$ noise data at $1 \mathrm{GHz}$, shown in figure 3-33, yielded an upper bound of about $150 \mu \mathrm{s}$ which is the limit of resolution for the number of data runs averaged.

Analysis of a limited number of "snapshot" runs suggests that the refresh time may be less than $50 \mu \mathrm{s}$. Further data should be collected and evaluated to obtain better resolution of the refresh time.

An EUT in a BLWGN-excited reverberation chamber will be effectively immersed in an isotropic and randomly polarized field on time scales of the BLWGN refresh time. Therefore, if the EUT response time is longer than the refresh time, it will not "see" the modal hot spots and nodes which occur with most mechanical mixing operations.

\section{EQUIPMENT UNDER TEST RESPONSES}

To demonstrate the feasibility of using BLWGN excitation of a reverberation chamber for radiated susceptibility and SE testing, several issues needed to be investigated. One issue was the ability of the BLWGN excitation to excite frequency-dependent or resonant responses in a EUT. This included the ability to excite responses from EUTs which have either narrow or broad susceptibility bandwidths. Another issue was how both peak and average power sensitive EUTs would respond to BLWGN excitation. Also EUTs whose susceptibility had an aspect angle dependence needed to be investigated. Finally shielding-effectiveness measurements with BLWGN excitation needed to be compared to results obtained with mechanical tuning.

Frequency-dependent responses were primarily investigated using standard transverse electromagnetic (TEM) cells. The theory of TEM cells, a description of the experimental set-up, and the results are discussed in section 4.1. A generic electronic system called the CTA (common test article), developed by NSWCDD and extensively tested using several test techniques, was used for the radiated susceptibility demonstration for peak power sensitive systems. A description of the CTA, the experimental set-up, and the results are covered in section 4.2. To demonstrate the response of an average power sensitive system, an instrumented $7 \mathrm{~cm}(2.75 \mathrm{in})$ folding-fin aircraft rocket (FFAR) was tested. The EUT description, the experimental set-up, and the results from BLWGN excitation and mechanical tuning in a reverberation chamber as well as anechoic chamber data are presented in 
section 4.3. Section 4.4 describes the SE tests and compares the results obtained from BLWGN and mechanical tuning in the reverberation chamber.

\subsection{TEM Cells}

\subsubsection{Theory of TEM Cells}

A cross section of a TEM cell is shown in figure 4-1. A circular aperture is centered in the upper wall, and the cell is tapered down to a coaxial line at each end. The line is terminated in a $50 \Omega$ impedance matched load at one end, and the power is received in an impedance matched, calibrated, power measurement detector at the other end.

Theory describing the aperture coupling of TEM cells is contained in refs. [16] and [17]. Briefly, for aperture coupling, this includes defining shielding effectiveness (SE) as

$$
S E=-10 \log \frac{A_{e}}{A_{r}}, d B
$$

where $A_{e}$ is the effective area of the apertured TEM cell (defined as the ratio of the received power $P_{r}$ picked up by the TEM cell to the incident power density $P_{d}$ to which the TEM cell is exposed, $A_{e}=$ $\mathrm{P}_{\mathrm{r}} / \mathrm{P}_{\mathrm{d}}$ ) and $\mathrm{A}_{\mathrm{r}}$ is the effective area of a reference antenna with known efficiency. The apertured TEM cell does not permit a rigorous analysis for determining its effective area because the external geometry is too complicated to compute the current density (tangential magnetic field) and charge density (normal electric field) incident on the aperture.

An approximate analysis for circular apertures [16] has been performed assuming that the fields incident on the aperture are equal to the free-space incident fields. Polarizability theory described in ref. [17] was used, along with a similar theory for excitation of the internal TEM mode by the aperture dipole moments. No averaging over incidence angles was done, but an expression for the maximum received power was derived. The maximum occurs when the incident field is propagating along the $\mathrm{z}$ axis with the electric field polarized in the $\mathrm{y}$ direction. The maximum effective area of the TEM cell is

$$
A_{e(\max )}=\frac{Z_{o}}{\eta}\left[\frac{16 r^{3}}{\lambda a \sinh (\pi b / 2 a)}\right]^{2},
$$

where the dimensions $\mathrm{a}$ and $\mathrm{b}$ are shown in figure 4-1. Since this theory deals only with the polarization matched case, $A_{e}$ is normalized by the effective area $\lambda / 4 \pi$ of an isotropic antenna to get shielding effectiveness

$$
S E=-10 \log \left(\pi Z_{d} / \eta\right)\left[\frac{32 r^{3}}{\lambda^{2} a \sinh (\pi b / 2 a)}\right]^{2}, d B
$$

The theory for calculating the cavity resonances of TEM cells is given in ref. [18]. A summary of the modal equations for calculating the frequencies for the first few higher order modes is given in 
table I, page 126 of ref. [18]. Once the cutoff frequency of a particular mode has been determined, the associated resonance frequencies $\mathrm{f}_{\mathrm{r}_{(\mathrm{m}, \mathrm{n}, \mathrm{p})}}$ can be calculated from

$$
\left[f_{r_{(m, n, p)}}\right]^{2}=\left[f_{c_{(m, n)}}\right]^{2}+\left[p c / 2 L_{m n}\right]^{2}
$$

where $f_{c_{(m, n)}}$ is the cutoff frequency of the mode, $L_{m n}$ is the effective length of the cell for the particular mode, and $\mathrm{m}, \mathrm{n}$, and $\mathrm{p}$ are integers.

The quality factor $Q$ of the resonances can be determined from their $\pm 3 \mathrm{~dB}$ bandwidths. If $\nabla \mathbf{f}$ equals distance between points on the response curve for which the amplitude response is down to $1 / 2$ of its maximum value (corresponding to $-3 \mathrm{~dB}$ ), then $\mathrm{Q}$ is given as

$$
Q=f_{l} / \Delta f
$$

where $f_{r}$ is the resonant frequency. $\Delta f=$ Bandwidth (BW), so eq (4-5) equals eq (3-7).

Results of calculations using table 1 of ref. [18] and eq (4-4) for the $12 \mathrm{~cm} \times 12 \mathrm{~cm}$ and $3 \mathrm{~cm}$ $\times 6 \mathrm{~cm}$ apertured TEM cells are given in tables 4.1 and 4.2 .

\subsubsection{Experimental Results}

Experimental results obtained using the NIST reverberating chamber with mechanical stirring for the two apertured TEM cells are shown in figures 4-2 and 4-3. The top curve on each figure is the measured SE of the particular cell with its aperture open. The smooth curves are the theoretically predicted SE calculated from eq (4-3). The lower curve on each figure is the measured SE with the cell's aperture covered with conductive tape. These data were taken to be sure the significant coupling was via the apertures. The sharp resonance spikes seen with the top and bottom measured curves are due to cavity resonances that occur in the particular cell. These can be compared with the resonance frequencies shown in the appropriate tables, 4.1 and 4.2. The agreement between measurement and theory is excellent for determining the resonant frequencies. The theoretical SE curves, however, do not agree with the measured values as well, probably because of the effects of the external geometry of the cells on the fields exciting their apertures. This seems apparent since the agreement improves as the size of the cell decreases.

The $\pm 3 \mathrm{~dB}$ bandwidth and quality factor of the measured resonances associated with the cells are also shown in tables 4.1 and 4.2. These data are given for comparison with the results of measurements made using the BLWGN technique to evaluate the cells as discussed in section 4.1.3.

\subsubsection{Explanation of Results and Coupling Cross Sections}

Coupling cross section is an important parameter that relates the power density incident on a system to the power delivered to a $50 \Omega$ instrument connected to a node of interest in the system under investigation. Coupling cross section is also often called effective area $A_{e}$ or effective aperture and it typically has the units of square centimeters. Cross section is defined as [20]: 


$$
\sigma(f)=\frac{\mathrm{P}_{\mathrm{L}}(f)}{\mathrm{S}_{\mathrm{inc}}(f)}=\frac{\eta_{o}\left|\mathrm{H}_{\mathrm{v}}(f)\right|^{2}}{R_{\mathrm{L}}},
$$

where $\sigma(\mathrm{f}), \mathrm{P}_{\mathrm{L}}, \mathrm{H}_{\mathrm{V}}(\mathrm{f}), \mathrm{R}_{\mathrm{L}}$, and $\mathrm{S}_{\text {inc }}(\mathrm{f})$ are the coupling cross section, power delivered to measuring instrument, voltage transfer function (voltage impressed at test point divided by the magnitude of the incident $\mathrm{E}$ field), input resistance of measuring instrument, and power density incident on system.

The first observation that can be made about eq (4-6) is that it assumes that the test object is being radiated with a plane wave. Secondly, it also assumes that the source of radiation is at a single frequency. Neither of these assumptions is correct in the case of BLWGN excitation. A B-dot probe or other polarized electromagnetic sensor inside a cavity with an isotropically impinging field will on the average measure only half of the power density associated with the field due to its polarization. Further, any directivity associated with the sensor will become 1 . In general this can also be said of a node inside the system being investigated [20]. Thus, the coupling cross section measured in a reverberation chamber will be reduced by both its directivity and polarization factor associated with the system regardless of how the modes are stirred. Although the polarization factor is easily corrected for, the directivity is much more difficult. For simple systems such as long slender rockets, the maximum directivity can be approximated from simple dipole models. If the maximum directivity at a given frequency cannot be modeled or measured in some other fashion, the coupling cross section as measured in a reverberation chamber should be considered as an average cross section over $4 \pi \mathrm{sr}$.

Next, we consider the effect of spreading the spectrum of the excitation source. Because the measuring instrument effectively measures the total average power being received by the node over the agility bandwidth, measuring the cross section using this method is equivalent to convoluting a gating function $\mathrm{g}$ with the narrow band cross section and then dividing by the power density, i.e.,

$$
\sigma\left(f_{c}\right)=\frac{\int_{f_{c}-B W / 2}^{f_{c}+B W / 2} g\left(f^{\prime}\right) \sigma\left(f^{\prime}-f_{c}\right) d f^{\prime}}{\int_{f_{c}-B W / 2}^{f_{c}+B W / 2} g(f) d f} .
$$

This has the same effect as mathematically smoothing $\sigma(\mathrm{f})$ over the same bandwidth. The primary difference is, however, that the smoothing is done in real time and is independent of the sampling resolution. In other words, if the sample interval were greater than the smoothing window, mathematical smoothing would not work, this method would, however.

As shown in figures 4-4 and 4-5, details in the cross section that span frequency intervals less than the agility bandwidth will tend to be washed out, while those that span greater intervals will remain. In fact, when the bandwidth of the resonance is less than the excitation bandwidth, the resonance will be reduced by the ratio of the resonance to the source bandwidth. This can be further seen in figures 4-6 and 4-7, which show the coupling cross section of the NIST standard $12 \mathrm{~cm}$ and $6 \mathrm{~cm}$ TEM cells, respectively [16]. Each cell has a small aperture through which energy couples. The cross section of each cell was measured with the BLWGN excitation technique using four 
different agility bandwidths $(2,10,20$, and $50 \mathrm{MHz})$. Note the resonance in figure 4-6 at approximately $0.925 \mathrm{GHz}$. This resonance is very narrow (on the order of $1 \mathrm{MHz}$ ). As the agility bandwidth is widened, the resonance appears to widen. In this case, because the cross-section bandwidth $\mathrm{BW}_{\mathrm{r}}$ is much less than the agility bandwidth $\mathrm{BW}_{\mathrm{g}}$ the resonance appears to have $\mathrm{Hx}$ bandwidth equal to the agility bandwidth, while its amplitude is decreased by the same amount the agility bandwidth is increased. The $6 \mathrm{~cm}$ TEM cell (fig. 4-7), on the other hand, has a resonance at approximately $6.45 \mathrm{GHz}$ with a bandwidth of around $100 \mathrm{MHz}$. Here, because all four agility bandwidths are less than the resonance bandwidth, the cross section as measured by each agility bandwidth is essentially the same and accurately reflects the true cross section less the polarization factor and directivity.

A comparison of figures 4-6 and 4-7 with figures 4-2 and 4-3 shows a match in the measured resonances for both cells between BLWGN and mechanical stirring excitation data, however, with some limitations. First, the BLWGN technique demonstrated the capability to find resonant modes with bandwidths much smaller than the BLWGN agility bandwidth although their amplitude is reduced. In figure 4-6, all the noise bandwidths located the narrow resonance identified in table 4.1 at $929 \mathrm{MHz}$ and shown in figure 4-2 for mechanical mode mixing. The dependence of the resonance amplitude on noise bandwidth is also evident in figure 4-6. Thus, assuming the measurement system has sufficient dynamic range to compensate for the decrease in response amplitude with bandwidth, an EUT resonance can be found for a wide range of bandwidths. Second, when the cross-section resonant bandwidth is greater than that of the BLWGN agility bandwidth, the spatial average cross section can be accurately measured. This can be seen in a comparison of the BLWGN at $6.4 \mathrm{GHz}$ in figure 4-7 with the mechanical mode-mixed data in figure 4-3. For this case there is excellent agreement between both the frequency and amplitude of the resonance between mechanical and BLWGN excitation.

\subsection{Electromagnetic Vulnerability Testing 4.2.1 Test Item Description}

The NSWCDD developed a simple electronic system to evaluate various susceptibility test techniques for repeatability and comparability. The system, referred to as the common test article (CTA), consists of three shielded boxes whose functions and interconnects are shown in figure 4-1. Boxes 1 and 2, which are battery powered and connected together via a combination of fiber optic cables and unshielded copper wires, are intended to be exposed to various electromagnetic environmental effects $\left(E^{3}\right)$. Box 3 , which is connected to boxes 1 and 2 via fiber optic cables and is ac powered, is used to monitor the "health" of the system and remotely test and/or reset the system. Box 1 consists of a $100 \mathrm{kHz}$ clock, a comparator circuit to compare two, 8-bit digital words, an error detection circuit which trips when the comparator circuit detects a mismatch, and a reset circuit that resets the counters and error detection circuit whenever an upset or test function occurs. Box 2 consists of two, 8-bit counters which simultaneously count the clock signal sent from box 1 and a test circuit which grounds one of the output lines of one counter, which causes the two counts to fail to match whenever the test function is activated. Box 3 consists of two fiber optic transmitters and one fiber optic receiver. The fiber optic receiver circuit monitors the system health and activates a green LED on the front of the box to indicate proper operation. If an upset occurs then a red LED is activated. The two fiber optic transmitter circuits send signals to the reset and test circuits whenever the corresponding button of the front of the box is pushed. Box 3 is also equipped with TTL compatible I/O to enable the system to be monitored, tested, and reset under computer control. 
Table 4.1. Mode cutoff and resonant frequency analysis of $12 \mathrm{~cm} \times 12 \mathrm{~cm}$ TEM cell.

\begin{tabular}{cccccc}
\hline Mode & $\begin{array}{c}\text { fc(m,n) } \\
(\mathrm{MHz})\end{array}$ & $\begin{array}{c}\text { Lmn } \\
(\mathrm{m})\end{array}$ & $\begin{array}{c}\text { Freq. } \\
(\mathrm{MHz})\end{array}$ & $\begin{array}{c}\mathrm{fr}(\mathrm{m}, \mathrm{n}, 1) \\
\mathrm{BW} \\
(\mathrm{MHz})\end{array}$ & $\mathrm{Q}$ \\
\hline TEO1 & 728 & 0.26 & 929 & 1.2 & 761 \\
TE10 & 1250 & 0.18 & 1502 & 3.2 & 470 \\
TE11 & 1563 & 0.16 & 1823 & 3.0 & 606 \\
\hline
\end{tabular}

Table 4.2. Mode cutoff and resonant frequency analysis of $3 \mathrm{~cm} \times 6 \mathrm{~cm}$ TEM cell.

\begin{tabular}{cccccc}
\hline Mode & $\begin{array}{c}\text { fc(m,n) } \\
(\mathrm{MHz})\end{array}$ & $\begin{array}{c}\text { Lmn } \\
(\mathrm{m})\end{array}$ & $\begin{array}{c}\text { Freq. } \\
(\mathrm{MHz})\end{array}$ & $\begin{array}{c}\text { fr(m,n,1) } \\
\text { BW } \\
(\mathrm{MHz})\end{array}$ & $\mathrm{Q}$ \\
\hline TEO1 & 3833 & 0.092 & 3269 & 5.9 & 556 \\
TE10 & 2679 & 0.080 & 3270 & 4.3 & 761 \\
TE11 & 4951 & 0.068 & 5420 & 3.0 & 1813 \\
TE20 & 5357 & 0.080 & 5676 & 2.0 & 2835 \\
TE21 & 6207 & 0.080 & 6484 & 2.0 & 3230 \\
\hline
\end{tabular}

Before this test, the susceptibility of the CTA was determined using CW excitation in the NSWCDD reverberation chamber. The results of the NSWCDD testing are shown in figure 4-9. The susceptibility of the CTA is low at $200 \mathrm{MHz}$, and the susceptibility level increases with frequency until about $2 \mathrm{GHz}$. Above $2 \mathrm{GHz}$, the CTA can withstand power densities above the arbitrarily imposed test limit of $10.61 \mathrm{~mW} / \mathrm{cm}^{2}$ which is equivalent to a field strength of $200 \mathrm{~V} / \mathrm{m}$.

Figure 4-10 shows the CTA set-up in a reverberation chamber with $2 \mathrm{~m}$ of cable between boxes 1 and 2. Previous testing of the prototype CTA have shown that variations in box position, orientation, and cable runs do not significantly affect the susceptibility test results obtained using a reverberation chamber.

All data signals, which pass from box 1 to box 2 by unshielded copper wires, pass through 74LS373 line drivers. These line drivers have a data set-up time of $5 \mathrm{~ns}$ which allows them to respond to inputs of very short duration.

\subsubsection{Experiment and Results}

The CTA was placed inside the NIST reverberation chamber on a styrofoam block as shown in figure 4-10. The measurement system was configured as shown in figure 4-11 for BLWGN excitation 
and as shown in figure 4-12 for $\mathrm{CW}$ excitation with mechanical stirring. The testing conducted determined the power density at which system upset would occur for both $\mathrm{CW}$ excitation and for noise excitation utilizing five different bandwidths of noise.

Since the previous susceptibility testing revealed that the susceptibility of the CTA increased with frequency, attempting to sweep would have resulted in upsets occurring at different power densities over the frequency sweep. This would result in having to "track" the vulnerability by altering both start frequency and power density. To eliminate this problem, testing was conducted at discrete frequencies for both methods of excitation. Testing was conducted at 19 frequencies over the frequency range from $100 \mathrm{MHz}$ to $2 \mathrm{GHz}$ and was limited to power densities not to exceed 10.61 $\mathrm{mW} / \mathrm{cm}^{2}$. At each of the discrete frequencies tested, the power density was initially set below the expected upset level, which was based on the previous CW testing conducted at NSWCDD. Once the initial power density was established, the power was increased in steps until an upset occurred or the power density limit was reached. For each failure the chamber input power was recorded and the average power density measured by each of the four probes (see section 3.1) shown in figures 4-11 and 4-12. A scalar network analyzer was used to measure the probe responses because its broad frequency response allowed it to respond accurately to both $\mathrm{CW}$ and narrow band noise stimulus. For $\mathrm{CW}$ excitation, the peak power density was also measured using a spectrum analyzer and a receiving antenna.

Before and after each test was conducted, the CTA was cycled by activating the test circuit and then executing a reset. This ensured that the CTA was still functioning properly and had not been damaged during the test.

\subsubsection{Band-Limited, White Gaussian Noise Excitation}

Testing began at $100 \mathrm{MHz}$ and proceeded upward in frequency. Once the initial power level was established, the power level was increased in $0.5 \mathrm{~dB}$ steps, with a $1 \mathrm{~s}$ dwell time at each step, until an upset occurred or the test limit was reached. At each failure the chamber input power was recorded and the average power density was measured by each of the four probes shown in figure 4-11. Only average power density was measured for the BLWGN measurements due to equipment limitations.

Data were collected for noise bandwidths of $2 \mathrm{MHz}, 4 \mathrm{MHz}, 10 \mathrm{MHz}, 20 \mathrm{MHz}$, and $50 \mathrm{MHz}$. The average upset power density as measured by the four probes for each of the five bandwidths tested is shown in figure 4-13. The data show that the upset level varied as much as $\pm 4 \mathrm{~dB}$ over the five bandwidths tested, with the average variation about $\pm 2 \mathrm{~dB}$. These variations are a sum of the input power variations caused by slight frequency dependent variations in the attenuations of the filters, cables, and couplers used and the field uniformity of the chamber for the various bandwidths. The largest variation was found to be at $100 \mathrm{MHz}$, while the smallest variations occurred at $500 \mathrm{MHz}$ and $1.4 \mathrm{GHz}$. At $1.8 \mathrm{GHz}$ an upset occurred using only the $20 \mathrm{MHz}$ bandwidth. The upset occurred at a power density of about $5 \mathrm{~mW} / \mathrm{cm}^{3}$ which is $3 \mathrm{~dB}$ below the maximum permitted power density. Based on the variation of the preceding data points it is possible that the failure levels for the other bandwidths could have been slightly above the maximum permitted power density without having variations greater than those already experienced. The data also show that the susceptibility levels were randomly distributed among the five bandwidths tested. This indicates no systematic bandwidth bias in the susceptibility results. 


\subsubsection{Continuous Wave Excitation Using Mechanical Stirring}

To check the results obtained using BLWGN excitation, data were collected using CW excitation and mechanical stirring at the same center frequencies used to collect BLWGN data. To collect the data, the test set-up was reconfigured as shown in figure 4-12. Data were collected by exciting the chamber while rotating the mechanical tuner $360^{\circ}$ over a $10 \mathrm{~s}$ period. The rotation time was selected to ensure that the time rate of change of the fields was slower than or equal to the response time of the spectrum analyzer used to measure the peak value of the fields. This also ensured that the effective dwell time afforded by the tuner rotation rate would be greater than the response time of the CTA. The power density was increased in $0.5 \mathrm{~dB}$ steps after each rotation of the mechanical tuner until an upset occurred or the $10.61 \mathrm{~mW} / \mathrm{cm}^{2}$ limit was reached. Initial power densities values were determined by subtracting $5 \mathrm{~dB}$ from the upset levels found using the BLWGN technique. When an upset occurred, the chamber input power was recorded, the peak power density was measured using a spectrum analyzer, and the average power density was measured using the four probes shown in figure 4-12. Each of the three readings was taken with the mechanical tuner in motion over a period corresponding to at least one complete rotation of mechanical tuner.

The peak and average power densities at which upset of the CTA occurred are shown in figure 414. The peak upset levels are consistent with the upset levels shown in figure 4-2, which were previously obtained using the NSWCDD reverberation chamber. The difference between the peak and average data is shown in figure 4-8. The difference between the peak and average upset levels varies from a high of $12.4 \mathrm{~dB}$ to a low of $3 \mathrm{~dB}$. The largest differences occur at the lower frequencies as should be expected since chamber uniformity decreases with frequency. The average difference is $7.7 \mathrm{~dB}$, which is consistent with the 7 to $8 \mathrm{~dB}$ variations previously discussed and with findings presented in refs. [6,19,21,22,23].

\subsubsection{Comparison of Noise Data to Continuous Wave Data}

Figure 4-16 combines the average of the BLWGN data shown in figure 4-13 with the CW data from figure 4-14. The data show an excellent correlation between the BLWGN upset levels and the average upset levels obtained using $\mathrm{CW}$ excitation and mechanical stirring. The difference between the CW average and the average BLWGN responses is shown in figure 4-19. The data indicate that the average BLWGN upset level was generally slightly higher than for the $\mathrm{CW}$ average. However two-thirds of the data points are within $\pm 3 \mathrm{~dB}$ and only one data point above $500 \mathrm{MHz}$ exceeded 3 $\mathrm{dB}$. These variations are within the expected chamber field uniformity as outlined in section 3.2.

The peak power upset level obtained using mechanical stirring is approximately $7 \mathrm{~dB}$ higher than the average power upset level using either mechanical stirring or the BLWGN. Since no peak data were collected for the BLWGN excitation, there are no data available for a direct comparison. However, as was discussed in section 3.3, the peak-to-average ratio for both BLWGN and mechanical mode mixing is typically 7 to $8 \mathrm{~dB}$. The difference between the peak of mechanically mode-mixed data and the average BLWGN data is shown in figure 4-18. The difference between the peak mechanical and average noise data varies from a high of $10.4 \mathrm{~dB}$ to a low of $3 \mathrm{~dB}$. The largest differences occur at the lower frequencies as should be expected since chamber uniformity decreases with frequency. The average difference is $6.5 \mathrm{~dB}$, which is consistent with the 7 to $8 \mathrm{~dB}$ variations previously discussed. The data are consistent with data presented in section 4.2.2.2, which were obtained using mechanical tuning alone. This indicates that the peak levels generated by the BLWGN are equivalent to the peak levels generated using mechanical tuning and that the CTA responded to the peak levels generated by the BLWGN. 
Testing was conducted for $1.8 \mathrm{GHz}$ using $\mathrm{CW}$ excitation, however no upset occurred. As stated in 4.2.2.1, an upset occurred when the chamber was excited with $20 \mathrm{MHz}$ noise bandwidth and no upset occurred at the other four bandwidths tested. Since the CW excitation did not cause upset at the $10.61 \mathrm{~mW} / \mathrm{cm}^{2}$ limit, the possibility that the upset level is within the variations previously discussed cannot be ruled out.

In summary, the fields generated by both methods have the same peak-to-average ratio and the two methods of excitation yield the same results when the data are properly interpreted. For this test the BLWGN excitation method was a factor of 10 faster than the mechanically tuned method. However, since the response time of the CTA was much shorter than the dwell times used for either excitation, and since the speed at which the fields can be changed is greater for the BLWGN than for mechanical mode mixing, it is likely that BLWGN excitation could be significantly faster than mechanically tuning when used for EMV testing.

\subsubsection{Comparison of Chamber Input Power Required for Upset}

Another way to determine the variation in the upset levels of the CTA is to examine the variation in chamber input power at which upset occurred for each excitation used. To accomplish this the average of the input power(s) at which upset occurred for each test frequency and bandwidth was calculated. The difference between the average input power and each individual input power was calculated. The results of these calculations are shown in figure 4-17. The data show that 97 percent of the data points fall within $\pm 3 \mathrm{~dB}$ and that 100 percent of the data fall within $\pm 4 \mathrm{~dB}$. The data also show no systematic bias due to either excitation method or bandwidth variations in BLWGN. The data did not converge or diverge with frequency indicating that the CTA responded the same to both excitation methods.

\subsection{Hazards of Electromagnetic Radiation to Ordnance Testing 4.3.1 Test Item Description}

The item tested, shown schematically in figure 4-1, was a modified, $7 \mathrm{~cm}$ (2.75 in) folding-fin aircraft rocket (FFAR) which consisted of a MK 66 MOD 1 rocket motor, MK 1 MOD 0 electric squib, and a warhead/fuse assembly. The electric squib is located in the forward end of the motor section and is enclosed in a thin-walled steel housing. Its function is to ignite the rocket motor propellent. The squib firing leads exit the igniter housing through a glass-insulating terminal. One firing lead connects to a wire that passes through the grain perforation, nozzle throat, and weather seal to connect with the contact band on the nozzle/fin assembly, while the other firing lead attaches to a spring assembly that grounds the lead to the motor case. The standard FFAR was modified for ease of instrumentation and safety as follows:

a. Rocket Motor-The MK 66 rocket motor propellant was removed and replaced with a spacer to maintain firing lead position. All other hardware was the same as the tactical rocket motor.

b. Electric Squib-The MK 1 MOD 0 electric squib was replaced by a MK 17 MOD 0 electric squib because of its known electrical/firing characteristics.

c. Warhead/Fuse-The warhead/fuse assembly was removed and replaced with a metal end cap which simulated the warhead/fuse attachment. 
d. Firing Lead-The firing lead was disconnected from the contact band and extended out of the nozzle. This increased the EM coupling to the rocket, which allowed all reverberation chamber testing to be conducted using only $1 \mathrm{~W}$ of input power to the chamber.

\subsubsection{Instrumentation}

For this test the MK 17 MOD 0 squib, which will be referred to as an EED, was monitored using a fiber optic multisensor system. The system, shown in figure 4-21, consisted of a measurement instrument, temperature sensor probes, fiber optic cables, and personality keys. The instrumented EED is shown in figure 4-22.

The measurement instrument may be viewed as a light transmitter and light receiver. The instrument sends light out four sensor ports simultaneously. The light propagates down an optical fiber until it reaches the sensor probe assembly's sensing element. The color of the transmitted light is altered by the sensing element as a function of the parameters it was designed to sense and reflected back to the instrument. The change in color (spectral change) of the light is measured by the instrument as it monitors the reflection from the sensor. The amount of color change is then compared to a calibration table stored in a memory device called a personality key. The resultant measurement information is presented on the front panel and is provided to an analog output on the rear of the instrument.

The measurement system has several advantages when used for monitoring EEDs which utilize hot bridgewires such as the one used in this test. These advantages include:

a. The measurement system is a self-contained unit which can be easily transported and operated.

b. The fiber optic cables can be of lengths approaching $2 \mathrm{~km}$ and can be used in most applications. The optical cables do not perturb the electromagnetic field nor does the electromagnetic field have an effect on the fiber cables.

c. The sensor probe assembly and sensing element are non-metallic and small compared to the EED. Therefore, the sensing element does not affect the energy induced into the EED's bridgewire. In addition, the sensing element is small enough that it may be placed in close proximity to or actually touching the bridgewire as shown in figure 4-22.

d. The measurement system can measure currents as low as $10 \mathrm{~mA}$ which is approximately $40 \mathrm{~dB}$ below the maximum no fire current (MNFC) of most EEDs.

e. The instrumentation's dynamic range is limited by the EED itself and the maximum repeated current allowed before damage will occur to the bridgewire of the EED. In this application, the dynamic range was approximately $34 \mathrm{~dB}$.

f. The measurement system response time varies between 25 and $90 \mathrm{~ms}$, which is slightly longer than the typical EED which has a thermal time constant of approximately 12 to $15 \mathrm{~ms}$. 


\subsubsection{Experiment and Results}

The FFAR was placed inside the NIST reverberation chamber on a styrofoam block and connected to the fiber optic measurement system as shown in figure 4-23. The testing conducted measured the amount of current induced in the bridgewire of the rocket's EED for approximately $1 \mathrm{~W}$ of rf energy injected into the chamber. Data were collected using the reverberation chamber for two types of excitation: (1) BLWGN, and (2) CW with mechanical tuning. Data were also collected using the NIST anechoic chamber with CW illumination for the purpose of comparing the reverberation chamber data to anechoic chamber data.

Before and after the test, the FFAR was calibrated by injecting a known direct current (dc) signal into the bridgewire leads and monitoring the output signal. This ensured that the characteristics of the EED did not change during the course of the test.

\subsubsection{Band-Limited, White Gaussian Noise Excitation}

Testing began with swept BLWGN excitation over the frequency ranges listed in table 4.3 , using the test set-up shown in figure 4-24. Sweep time for each frequency range was $200 \mathrm{~s}$. This ensured that the EED would have time to respond to any current induced in the bridgewire. Testing was conducted using $2 \mathrm{MHz}, 4 \mathrm{MHz}, 10 \mathrm{MHz}, 20 \mathrm{MHz}$, and $50 \mathrm{MHz}$ noise bandwidths. The data collected are shown in figures 4-25 through 4-28. The data show multiple narrow, well defined resonances. The amplitudes of the responses vary greatly with bandwidth at frequencies below 600 $\mathrm{MHz}$. At frequencies above $600 \mathrm{MHz}$ the amplitude variations caused by changing the bandwidth decrease as frequency increases. This indicates that the bandwidths of the resonances at the lower frequencies, such as at $450 \mathrm{MHz}$, are narrower than the noise bandwidths. However, the bandwidths of the resonances at the higher frequencies, above $2.1 \mathrm{GHz}$, are increasing with frequency approaching the width of the noise bandwidths. These bandwidth effects are consistent with the theory outlined in chapter 3 and section 4.1 .

The data also show a resonance at approximately $150 \mathrm{MHz}$, which is below the chamber's lowest recommended usable frequency. At this frequency, the chamber has only 28 modes, considerably less than the 60 or more modes generally accepted [6]. This suggests the possibility that the lower frequency limit of a reverberation chamber may be extended using BLWGN excitation as opposed to $\mathrm{CW}$ excitation with mechanical tuning for determining resonance characteristics of the device under test.

\subsubsection{Continuous Wave Excitation Using Mechanical Tuning}

To check the results obtained with BLWGN excitation, data were collected over a limited frequency range using $\mathrm{CW}$ excitation with mechanical stirring. The frequency range investigated was from $500 \mathrm{MHz}$ to $650 \mathrm{MHz}$ and was selected based on the BLWGN data. The test set-up was reconfigured as shown in figure 4-29. Data were collected by sweeping over the frequency range at each of 201 discrete tuner positions required to rotate the tuner through $360^{\circ}$ of rotation. Sweep time was maintained at $200 \mathrm{~s}$ per sweep. After all 201 sweeps had been collected and saved on disk, the data were analyzed to determine the maximum and average responses of the rocket. These responses are shown in figure 4-30. The difference between the maximum and average data is approximately 7 $\mathrm{dB}$. This is consistent with data shown in ref. [6] and earlier in this report. 
Table 4.3. Frequencies ranges for swept BLWGN excitation testing of folding-fin aircraft rocket.

\begin{tabular}{lll}
\hline \multicolumn{3}{c}{ Frequency $(\mathrm{GHz})$} \\
\hline 0.05 & - & 0.5 \\
0.5 & - & 1 \\
1 & - & 2 \\
2 & - & 4 \\
\hline
\end{tabular}

\subsubsection{Comparison of Noise Data to Continuous Wave Data}

The average data obtained using $\mathrm{CW}$ excitation with mechanical tuning and $2 \mathrm{MHz}$ BLWGN are compared in figure 4-31. The data show excellent correlation. Again, the maximum response obtained using the mechanical method is approximately $7 \mathrm{~dB}$ higher than the BLWGN response. This is as expected assuming the EED response time is less than the test field exposure dwell time. (The EED's response time is approximately $15 \mathrm{~ms}$ ). The mechanical stirring method causes frequency dependent maximums to exist at various locations through the test volume of the chamber as a function of tuner position. Each of these maximums exists for a period of time consistent with the dwell time afforded by the frequency sweep time. If this is greater than the EED's response time, the EED will respond to the maximum field. The maximum fields generated by BLWGN excitation exist for time intervals measured in microseconds, as discussed in section 3.3. Thus, the EED responds to the BLWGN averaged field present within the chamber.

In summary, the two methods of excitation yielded the same results when the data are properly interpreted. The BLWGN excitation method was on the order of 200 times faster than the mechanically tuned method. However, for a given chamber at a given input power, the mechanically tuned CW excitation method will effectively deliver approximately $7 \mathrm{~dB}$ more power to an average power sensitive device than can be obtained using BLWGN.

\subsubsection{Anechoic Chamber Data}

To complete the characterization in an anechoic chamber of the 2.75 -in rocket, an evaluation was conducted over the same $500 \mathrm{MHz}$ to $650 \mathrm{MHz}$ frequency range. The rocket was mounted in the NIST anechoic chamber as shown in figures 4-32. The block diagram of the test system is shown in figure 4-33. Initially, discrete frequency data were collected at four frequencies between $500 \mathrm{MHz}$ and $650 \mathrm{MHz}$. Two frequencies were selected from the first peak, one frequency from between the peaks (a low point) and one frequency from the second peak. Data were collected at each of the four frequencies using horizontal polarization at five roll angles, between 0 and $180^{\circ}$, as the rocket was rotated $360^{\circ}$ in azimuth. Data were also collected at two frequencies corresponding to the "peaks" using vertical polarization at roll angles of 0,45 , and $90^{\circ}$. The power density in the anechoic chamber was set the same as the average power density obtained in the reverberation chamber using 1 W of input power. These data are shown in figures 4-34 through 4-38. As would be expected, the data show that minimal response was obtained when using vertical polarization and that maximum response was obtained using horizontal polarization. Based on the limited number of aspect angles evaluated, $0^{\circ}$ roll at an azimuth angle of $65^{\circ}$ was determined to be the maximum response orientation. The measurement system was reconfigured as shown in figure 4-39, to collect swept frequency data at the maximum response orientation. Again, the power density in the anechoic 
chamber was the same as the average power density obtained in the reverberation chamber. The response obtained is shown in figure 4-40.

\subsubsection{Comparison to Anechoic Chamber Data}

The anechoic chamber data verify the presence of the two resonances found in the reverberation chamber between 500 and $650 \mathrm{MHz}$. A comparison of the anechoic chamber response to the $2 \mathrm{MHz}$ bandwidth noise response is shown in figure 4-41. As expected, the anechoic chamber response was higher than the reverberation chamber response [6]. If we apply a polarizability factor of 2 to the rocket's response data, as discussed in section 3.1, and correct for the reverberation chamber data accordingly, as shown in figure 4-42, we see that the response obtained from the reverberation chamber is still less than the anechoic chamber response. The remaining difference is attributed to the directivity of the device [6]. In this case, the difference was about $1.8 \mathrm{~dB}$, or roughly equivalent to a dipole antenna which has a directivity of $2 \mathrm{~dB}$. As previously described, the configuration of the firing lead was approximately a short dipole. Thus, this example demonstrates the loss of directivity in a reverberating chamber and illustrates that if the directivity of a test item is known or can be estimated, the responses obtained using the two types of facilities can be correlated.

An anechoic chamber test is the best simulation for an item immersed in a plane wave, free field environment, while a reverberation chamber best represents an item immersed in a complex environment such as that encountered in an enclosed cavity.

Also, the power necessary to generate the required power density in the anechoic chamber was approximately $10 \mathrm{~W}$ or $10 \mathrm{~dB}$ more than for the reverberation chamber.

\subsection{Shielding Effectiveness Testing of Materials 4.4.1 Shielding Theory}

Shielding effectiveness measurements using reverberation chambers are typically performed using nested chambers, that is, a reverberation chamber within a reverberation chamber. The technique is shown in figure 4-43.

Two approaches have been used for SE measurements. In one approach, the ratio of the received power in the fixture to the received power in the large chamber yields the SE. In the second approach, the ratio of the received power in the fixture with the sample removed to the received power in the fixture with the sample in place yields the SE.

Several factors that depend on the test fixture itself could influence the measured SE values. These factors include the fixture size and Q, aperture effects, and the sample mounting techniques. Work is in progress at NIST, NSWCDD, and PL to address these issues both theoretically and experimentally.

To investigate the applicability of the BLWGN technique to SE measurements, a direct comparison was performed to the SE results obtained with mechanical mode mixing for a wire mesh window assembly. 


\subsubsection{Test Item Description}

SE measurements were conducted on a $17.8 \mathrm{~cm} \times 10.2 \mathrm{~cm}$ shielded window shown in figure 444. Shielding was provided by a fine wire mesh embedded in the window which was terminated to a wire mesh gasket. The window was mounted over a $25.4 \mathrm{~cm} \times 15.2 \mathrm{~cm}$ opening in the top of a 45.7 $\mathrm{cm} \times 45.7 \mathrm{~cm} \times 45.7 \mathrm{~cm}$ aluminum box test fixture shown in figure $4-45$. The test fixture is sectioned into two compartments to provide isolation between the receiving antenna, which was mounted in the larger upper compartment, and a dc motor, which was mounted in the lower compartment. The drive shaft of the dc motor protrudes into the upper compartment where it connects to the paddle wheel tuner.

Since the shielded window is smaller than the aperture in the top of the test fixture, an adaptor plate was used to mount the window to the fixture. The window was attached to the adaptor plate using fourteen 6-32 UNC screws as shown in figure 4-46 and remained attached to the adapter plate for the duration of the test. This was done to prevent mounting repeatability problems from affecting the test results. (The actual SE measured was that of the window assembly and not necessarily that of the window itself. References to the SE of shielded window refer to the SE of the entire assembly.)

\subsubsection{Experiment and Results}

The test fixture was placed in the NIST reverberation chamber on a styrofoam block as shown in figure 4-47. The measurement system block diagrams are shown in figures 4-48 and 4-49 for CW excitation (mechanical stirring) and for BLWGN excitation. The tests required measuring the power density inside both reverberation chambers (the NIST chamber and the test fixture) with and without the shielded window installed. The tuners were stationary for the BLWGN measurements. The data collection process consisted of three separate measurements. They were:

1. Baseline Data: These were data collected with essentially no shielding of the receiving antenna present. For this test the shielded window was removed from the test fixture.

2. Shielding Data: These were data collected with the shielded window mounted to the test fixture. The difference between these data and the baseline data is the SE.

3. Dynamic Range Data: These were data with the shielded window covered with aluminum foil and aluminum tape. The difference between these data and the baseline data should be greater than the difference between the shielding data and the baseline data. This ensured that the detected signal entered through the shielded window or its associated gasket, and not through leakage through the test fixture or associated connectors, cabling, etc.

\subsubsection{Band-Limited, White Gaussian Noise Excitation}

Testing began with collection of data at $2 \mathrm{MHz}, 10 \mathrm{MHz}$, and $50 \mathrm{MHz}$ noise bandwidths over the frequency range of $1.25 \mathrm{GHz}$ to $6 \mathrm{GHz}$ to determine what effect various bandwidths of noise would have on shielding effectiveness measurements. For completeness, one data run to $18 \mathrm{GHz}$ was performed using $10 \mathrm{MHz}$ noise bandwidth. The data shown in figures 4-50 through 4-52 reveal that the shielding effectiveness smooths out as noise bandwidth increases. This "smoothing" is due to averaging the shielding over the bandwidth and the improving field uniformity with increasing bandwidth. (The contribution attributed to each effect is an area for further study.) [ The sharp decrease in shielding at approximately $3 \mathrm{GHz}$ was detected by all three bandwidths, but is most 
pronounced in the $10 \mathrm{MHz}$ bandwidth data. These data give the best comparison with data obtained previously for the shielded window [24]. Unfortunately, the mating surface of the adaptor plate had not been properly prepared for the BLWGN measurements which resulted in less shielding being provided by the window. For this reason, these data should be used only to examine bandwidth effects and should not be compared in an absolute sense to other data presented for the shielded window.

Measurements with $10 \mathrm{MHz}$ bandwidth BLWGN were repeated after properly preparing the mating surface of the adaptor plate and reattaching the shielded window.

Baseline data for the measurements were collected from both the large chamber and from the test fixture. As stated in section 4.4.1, SE measurements can be made using baseline data obtained from either the large chamber or from inside the test fixture. The results of the baseline measurements are shown in figure 4-53. These data show an offset of approximately $4 \mathrm{~dB}$ between the received powers of the chamber and the test fixture with the aperture open. This is due to the differences in cable attenuations and the efficiencies of the two chambers' receiving antennas. This indicates that if the shielding effectiveness were determined by taking the difference between the chamber antenna and the test fixture antenna when the shielded window was installed, a correction factor of approximately 4 $\mathrm{dB}$ would need to be added to the shielding data to correct for the differences between the two receiving systems. The data also indicate that the data collected by the test fixture antenna were not affected by waveguide below cutoff effects caused by the aperture in the test fixture.

SE data for the window were obtained by measuring the difference between the power received by the test fixture antenna with and without the window mounted. The results of these measurements corrected for the approximately $4 \mathrm{~dB}$ difference previously mentioned are shown in figure 4-54. These data agree well $( \pm 2 \mathrm{~dB})$ with previously obtained results.

Dynamic range data were collected by covering the shielded window with aluminum foil and taping the edges to the adaptor plate using aluminum tape. This yielded a minimum of $10 \mathrm{~dB}$ reduction in received power across the frequency range tested indicating that the shielding measured was due to coupling through the window not leakage through cables and other ports of entry.

The average data collection time using the BLWGN method was about 1 min per sweep.

\subsubsection{Continuous Wave Excitation Using Mechanical Tuning}

To check the results obtained using BLWGN excitation, data were collected using CW excitation and mechanical tuning over the 2 to $18 \mathrm{GHz}$ frequency range. To collect the data, the test set-up was reconfigured as shown in figure 4-48. The rotation rate of the test fixture tuner was set to $1 \mathrm{rev} / \mathrm{s}$ and the rotation rate of the chamber tuner was set to $6 \mathrm{rev} / \mathrm{min}$. The spectrum analyzer was set to sweep the 2 to $18 \mathrm{GHz}$ frequency range over a $60 \mathrm{~ms}$ time and placed in a "max-hold" mode while the rf synthesizer was set to continuously sweep over the 2 to $18 \mathrm{GHz}$ frequency range at the rate of 200 s per sweep.

Baseline data were collected by removing the window from the test fixture and collecting data for a period of approximately one hour.

Shielding data were collected by installing the shielded window/adaptor plate to the test fixture and repeating the data collection process for a period of approximately one hour. 
The difference between the baseline data and the shielding data yields the SE of the window shown in figure 4-54.

\subsubsection{Comparison of BLWGN Data to Continuous Wave Data}

The difference between the CW and BLWGN data is shown in figure 4-55. The data indicate reasonable agreement for the SE measured by both techniques. The difference between the $\mathrm{CW}$ data (smoothing factor 5 percent) and the BLWGN data is shown in figure 4-56. Although the maximum difference in the data shown in figure 4-55 is about $20 \mathrm{~dB}$, the smoothed data in figure 4-56 show variations on the order of $\pm 2 \mathrm{~dB}$. The data also show an offset of about $2 \mathrm{~dB}$, indicating that the BLWGN yielded slightly higher SE than did the CW excitation with mechanical mixing. This offset could be caused by several factors and should be studied further. In addition, the smoothing of the $\mathrm{CW}$ data assumes that the majority of the structure in the CW data is due to variations in field uniformity. Determining whether smoothing should be applied, as well as how much, is also a subject for further study.

In summary, $\mathrm{SE}$ data obtained using $\mathrm{BLWGN}$ or $\mathrm{CW}$ excitation with mechanical mode mixing yield comparable results. The notable difference is the "smoothing" effect of the BLWGN technique. The time required to collect SE data using the BLWGN technique however, is about one onehundredth of that required for $\mathrm{CW}$ excitation using mechanical tuning. With the two-antenna method, differences in the efficiency of the receiving antennas and cable losses used in the test fixture and outer chamber must be properly corrected for.

\section{SPECIAL CONSIDERATIONS ASSOCIATED WITH TESTING WITH BLWGN EXCITATION \\ 5.1 Instrumentation and Probes}

The EME associated with mechanically stirred or BLWGN excited reverberating chambers are quite different. Time constants associated with variations in the test fields are determined by either the rotation rates and hence mechanical redistribution of the fields stirred by tuners, or the modulation rates of the BLWGN system. In each technique, the fill or relaxation time of the reverberation chamber cavity can also affect the test field EME. Some types of instrumentation are capable of responding to very short time constants, for example probes such as B-dot or D-dot, and diode detectors/sensors. However, some types of probes, for example, electrically short dipoles with high resistance lines and distributed filters, have relatively slow response times (milliseconds). Also, some instrumentation has limited resolution bandwidth detection systems, for example, spectrum analyzers, and hence, cannot be used with BLWGN bandwidths that are significantly greater. This is the reason scalar analyzers were used with the BLWGN excitation measurement system. The need to understand and determine the impact of these measurement limitations was the motivation for the first phase of this study. The results of that study verified that only calibrated rf probes such as the B-dot, D-dot, or log periodic or horn antennas should be used to measure the test field for the BLWGN excitation technique. Essentially all types of good quality, calibratable probes can be used to measure the test field using mechanical stirring. The study also verified that spectrum analyzers with sufficient resolution bandwidths (bandwidths greater that the BLWGN excitation bandwidths) could be used, for example, a $3 \mathrm{MHz}$ resolution bandwidth for BLWGN bandwidths less than $2 \mathrm{MHz}$. Otherwise, spectrum analyzers should not be used. 
The limitations discussed above raise the question, "How will the EUT respond in the EME of the two techniques?" If the EUT's response is very fast (microseconds or less) it will track the peak of the exposure EME while the BLWGN excitations instrumentation will measure average values. Hence, this effect must be accounted for in evaluating EUT responses.

\subsection{Mixers}

Since microwave mixers are fundamental to the generation of BLWGN excitation, consideration must be given to the way they are used. Improper use of mixers will give erroneous results which are difficult to distinguish from real EUT responses. The mixers used in this experimental series were a special type called up-converters or balanced modulators. They were selected for their good carrier suppression, low VSWR, and low conversion loss. Practical considerations for using these mixers include input dynamic range, intermediate frequency (IF) to local oscillator (LO) isolation, and IF contained on rf output.

It is desirable to vary the level of the BLWGN excitation signal over a large range so that EUTs with varying degrees of upset sensitivity can be tested. The test configuration described earlier for generating BLWGN excitation relies on the variability of the synthesized sweeper to accomplish this. In other words, the LO input to the modulator is varied to create corresponding variation in output power level. Practical modulators, however, rely on the nonlinear properties of diodes to accomplish frequency conversion. The diodes must be operated in a region defined at their low sensitivity end by the noise level of the diode and at their high output end where they respond in a linear fashion. Hence, the synthesized sweeper must maintain an input level to the modulator that does not vary outside this range. The modulators used in this series were designed to operate best with an IF input of $+10 \mathrm{dBm}$ and a LO input of typically $-5 \mathrm{dBm}$. The IF input from the noise source was held constant by setting the noise source's internal attenuation using the following equation:

$$
A=20-10 L O G \frac{L P_{B W}}{100 \times 10^{6}}
$$

This equation was determined by noting that the full power output of the noise source is $30 \mathrm{dBm}$ and that this power is reduced by the ratio of the low pass filter bandwidth $\mathrm{LP}_{\mathrm{BW}}$ to the full bandwidth of the source, $100 \mathrm{MHz}$. For example, if the BLWGN excitation bandwidth is $10 \mathrm{MHz}$, the low pass filter would have a bandwidth of $5 \mathrm{MHz}$ and, thus, the internal attenuator would be set to $7 \mathrm{~dB}$. The LO input was varied over a relatively large range $(-20$ to $0 \mathrm{dBm})$, but care was taken to monitor the BLWGN excitation signal to ensure good suppression of the rf carrier and good SNR. A better system would use a step attenuator and small signal amplifier after the mixers to allow the LO input to be maintained at the optimal level for the mixer while the output power is controlled by the step attenuator. This would allow maximum dynamic range.

Mixers designed to have low input VSWRs typically do so at the expense of IF to LO isolation. Some of the IF power is transmitted back out the LO input and ends up at the synthesized sweeper output port. If internal leveling is being used on the sweeper, the IF signal being fed back can be interpreted as sweeper output power causing the sweeper to generate less rf. One way to reduce this problem is to place some attenuation between the mixer and the sweeper and increase the sweeper output by the same amount. For example, if a $10 \mathrm{~dB}$ attenuator is placed between the synthesizer and the modulator, the synthesizer output can be increased to $+5 \mathrm{dBm}$ (to give $-5 \mathrm{dBm}$ at the mixer). 
The IF being transmitted back will be reduced by $10 \mathrm{~dB}$ while the sweeper output was increased by $10 \mathrm{~dB}$, thus, allowing a $20 \mathrm{~dB}$ improvement in SNR for the leveling circuits. In this experimental series, a $6 \mathrm{~dB}$ splitter was placed between the sweeper and the mixer to monitor the sweeper output. Thus, a $12 \mathrm{~dB}$ improvement was inherent in the set-up.

The final issue that must be considered when using mixers for BLWGN excitation is the IF and, to a lesser extent, the higher harmonics of the LO present on the rf output port. The harmonics generated by the TWT amplifiers are usually much more significant. When the signal is fed through a band-limited amplifier, such as a TWT, and then through a band-limited antenna, the IF is inherently filtered out. When measuring the signal directly out of the mixer or when using it for external leveling, however, the IF can have a significant effect. The detector heads used on the scaler analyzer have a lower frequency range of $10 \mathrm{MHz}$ and, hence, for wider bandwidth BLWGN excitation they can pick up the IF signal which results in erroneous readings. Likewise, detector diodes used for external leveling experience the same problem. Certain low power applications where very broad band amplifiers with low cutoff frequencies are used can further aggravate the problem by amplifying the IF. This problem can be corrected by using a high pass filter with a lower cutoff range below the desired BLWGN excitation frequency but above the IF bandwidth.

Figure 5-1 shows the suggested improvements mentioned above. The $10 \mathrm{~dB}$ attenuator improves isolation between the IF signal and the synthesized sweeper. The high pass filter eliminates the IF signal from appearing in the output. It should be noted that a high pass filter could also be used in place of the $10 \mathrm{~dB}$ attenuator if sufficient power is not available from the sweeper. And finally the small signal amplifier boosts the signal for input to a TWT while the computer or voltage controlled attenuator allows varying the output level over any desired dynamic range.

\section{CONCLUSIONS}

\subsection{Chamber Environmental Characterization}

\subsubsection{Effective Mode Density}

At low frequencies where the total number of modes within the span of the $Q$ bandwidth is small, the effective mode density is essentially equivalent to the theoretical mode density. At higher frequencies, where many modes are encompassed by the span of the $\mathrm{Q}$ bandwidth, effective mode density becomes dependent upon the ratio of noise bandwidth to $\mathrm{Q}$ bandwidth.

\subsubsection{Field Uniformity}

In general, as described above, a reverberation chamber has two separate frequency regimes that determine the ability of either BLWGN excitation or mechanical mode perturbation to provide field uniformity. In both regimes, mechanical mode mixing relies upon physical stirrer size, while BLWGN excitation depends on noise bandwidth. For a given mechanical tuner and test configuration, an equivalent field uniformity can be achieved by selecting an appropriate noise bandwidth.

\subsubsection{Field Peak-to-Average Ratio}

The peak-to-average field ratio for both mechanical and BLWGN excitation are equivalent and are approximately 7 to $8 \mathrm{~dB}$ for sufficiently high-Q cavities. For a given level of mode mixing with 
either mechanical mode mixing or BLWGN, the peak-to-average ratio in a heavily loaded (low Q) chamber can be significantly less than the typical range of 7 to $8 \mathrm{~dB}$.

\subsubsection{Time Between Peak Field Excitation}

For mechanical mode mixing the EME refresh time is determined by the tuner rotation rate and is typically on the order of seconds. In the NIST reverberation chamber, the peak field refresh time for BLWGN excitation was less than $150 \mathrm{~ms}$ and could be as short as a few tens of microseconds.

\subsection{Test Applications}

Based on the three EUTs tested, the following conclusions were reached.

\subsubsection{Peak Power Sensitive EUTs}

Failure levels for BLWGN excitation are equivalent to failure levels for mechanical mode mixing for peak power sensitive EUTs.

\subsubsection{Average Power Sensitive EUTs}

For average power sensitive EUTs there is an apparent 7 to $8 \mathrm{~dB}$ difference in failure levels. This is based on the difference in cavity EME for the two test procedures. During a mode-tuned procedure, for some tuner position(s) the EUT will be exposed to the maximum field for the tuner dwell time. Thus, an average power sensitive EUT will fail at a level determined by the peak field in the chamber when a mode-tuned procedure is used: When the chamber is excited with BLWGN whose bandwidth is less than the susceptibility bandwidth of the EUT, the failure level will be determined by the average power in the chamber. As noted earlier, the peak-to-average power ratios for the two techniques are equivalent and are approximately 7 to $8 \mathrm{~dB}$. If the EUT susceptibility bandwidth is narrower than the noise bandwidth, the measured failure level will be increased by the ratio of the noise to susceptibility bandwidth.

\subsubsection{Shielding Effectiveness Measurements}

Shielding effectiveness measurement results for BLWGN excitation are equivalent to mechanical stirring results provided resonances in the shielding transfer function are wider than the noise bandwidth. Shielding transfer function features which are narrower than the noise bandwidth will be averaged over the noise bandwidth.

\subsection{Test Considerations}

1. Testing using noise excitation requires consideration of several instrumentation and procedural issues which may be different from the issues associated with mechanical stirring.

2. Using BLWGN test time requirements using noise excitation are reduced by a factor up to 200 compared to a conventional mechanical stirring method.

3. Test EUTs are exposed to fields which are uniform on time scales on the order of tens of microseconds. 
4. Most methods of measuring chamber field strength may be used for either technique. However, high resistance line sensors should not be used with BLWGN excitation. In lieu of typically used reference antennas such as log periodics and horns, smaller magnetic and electric field sensors such as B-dot and D-dot probes can be used to reduce chamber loading.

5. Power stacking or combining is achievable using both mechanical stirring and BLWGN excitation techniques.

\section{RECOMMENDATIONS}

Extend the comparison of mechanical and BLWGN mode-mixing techniques to further investigate the characterization of cavity uniformity by the two techniques.

Investigate how devices with narrow susceptibility bandwidths affect "effective" cavity uniformity.

Continue the validation of the BLWGN technique for radiated immunity testing by investigating a broader class of test systems.

Extend the comparison of the BLWGN technique to additional established radiated techniques such as open area test sites, shielded enclosures, GTEM cells, semi-anechoic and anechoic chambers.

Investigate the ability to employ a broad range of modulations with the BLWGN technique.

Investigate the application of BLWGN to small and/or densely packed cavities in which it is impractical to accommodate an adequately sized mechanical tuner.

Continue the theoretical and experimental investigation necessary to resolve the effects of aperture size, window-to-aperture interface, and fixture size and $Q$ effects on shielding effectiveness measurements using nested reverberation chambers.

Evaluate the feasibility of simulating the effects of plane wave illumination of large test articles using multiple antennas driven by incoherent BLWGN.

\section{REFERENCES}

[1] Mendez, H.A.; A new approach to electromagnetic field-strength measurements in shielded enclosures. Wescon Technical Papers, Western Electronic Show and Convention: Los Angeles, CA; Aug. 20-23, 1968.

[2] Bean, J.L.; Hall, R.A.; Electromagnetic susceptibility measurements using a mode stirred chamber. IEEE Intl. Symp. on Electromagnetic Compatibility; Atlanta, GA; June 1978.

[3] Corona, P.; Latmiral, G.; Paolini, E.; Piccioli, L.; Use of a reverberating enclosure for measurement of radiated power in the microwave range. IEEE Trans. Electromagn. Compat. EMC-18(2): 54-59, May 1976. 
[4] Cummings, J.R.; Translational electromagnetic environment chamber, A mew method for measuring radiated susceptibility and emissions. IEEE Intl. Symp. on Electromagnetic Compatibility; San Antonio, TX; Oct. 1975.

[5] Roe, J.M.; An improved technological basis for radiated susceptibility and emissions. IEEE Intl. Symp. on Electromagnetic Compatibility; San Antonio, TX; Oct. 1975.

[6] Crawford, M.L.; Koepke, G.H.; Design evaluation and use of a reverberation chamber for performing electromagnetic susceptibility/vulnerability measurements. Nat. Bur. Stand (U.S.) Tech. Note 1092; April 1986.

[7] Loughry, T.A.; Frequency stirring: An alternate approach to mechanical mode-stirring for the conduct of electromagnetic susceptibility testing. Phillips Laboratory, Kirtland AFB, NM; Report No. PL-TR-91-1036; Nov. 1991.

[8] Carlson, A.B.; Communication Systems, An Introduction to Signals and Noise in Electrical Communication. McGraw-Hill, New York, NY; 1975.

[9] Mory, R.; et al.; Development and production of multi-gap loop (MGL) series EMP B-dot. Report No. AFWL-TR-70-153; Air Force Weapons Laboratory; Feb. 1971.

[10] Olsen, S.; Asymptotic conical dipole D-dot sensor (ACD-S1(R)) development. Report No. AFWL-TR-75-263; Air Force Weapons Laboratory; May 1977.

[11] Pozar, D.M.; Microwave Engineering. Addison-Wesley Publishing Co., Reading, MA; 1990.

[12] Whiteside, H.; King, W.P.; The loop antenna as a probe. IEEE Trans. Antennas Propagat. AP-12(3): 291-297; May 1964.

[13] Richardson, R.E.; Mode-Stirred Chamber Calibration Factor, Relaxation Time, and Scaling Laws. IEEE Trans. Instrum. Meas. IM-34(4); Dec. 1985.

[14] Hatfield, M.O.; Freyer, G.J.; Johnson, D.M.; Farthing, C.L.; Demonstration test of the electromagnetic reverberation characteristics of a transport size aircraft, Naval Surface Warfare Center, Dahlgren Division, Dahlgren, VA; Report No. NSWCDD/TR-93/339.

[15] Jackson, G.A.; Smithers, B.W.; Review of EMC methods using mode stirred enclosures. Electromagnetic Compatibility Conf., University of Surrey, London; Sept. 1984.

[16] Hill, D.A.; Crawford, M.L.; Kanda, M.; Wu, D.I.; Aperture coupling to shielded transmission lines: theory and experiment. Natl. Inst. Stand. Technol. NISTIR 3988; Apr. 1992.

[17] Wilson, P.F.; Ma, M.T.; A study of techniques for measuring the electromagnetic shielding effectiveness of materials. Nat. Bur. Stand. (U.S.) Tech Note 1095; May 1986.

[18] Wilson, P.F.; Ma, M.T.; Simple approximate expressions for higher order mode cutoff and resonant frequencies in TEM cells. IEEE Trans. Electromagn. Compat. EMC-28(3): 125130; Aug. 1986. 
[19] Department of Defense Methodology Guidelines for High Power Microwave (HPM) Susceptibility Assessments, Office of the Secretary of Defense; Jan. 1990.

[20] Tai, C.T.; On the definition of the effective aperture of antennas. IEEE Trans. Antennas Propagat. AP-9: 224-225; Mar. 1961.

[21] Crawford, M.L.; Koepke, G.H.; Electromagnetic radiation test facilities: Evaluation of reverberation chambers located at NSWCDD, Dahlgren, VA. Nat. Bur. Stand. (U.S.) NBSIR 86-3051; June 1986.

[22] Crawford, M.L.; Koepke, G.H.; Ladbury, J.M.; EMR test facilities - Evaluation of reverberating chamber located at RADC, Griffiss AFB, Rome, NY. Nat. Bur. Stand. (U.S.) NBSIR 87-3080; Dec. 1987.

[23] Crawford, M.L.; Ma, M.T.; Ladbury, J.M.; Riddle, B.F.; Measurement and evaluation of a TEM/reverberating chamber. Natl. Inst. Stand. Technol. Tech. Note 1342, July 1990.

[24] Hatfield, M.O.; Shielding effectiveness measurements using mode-stirred chambers: A comparison of two approaches. IEEE Trans. Electromagn. Compat. EMC-30(3): 229-238; Aug. 1988. 


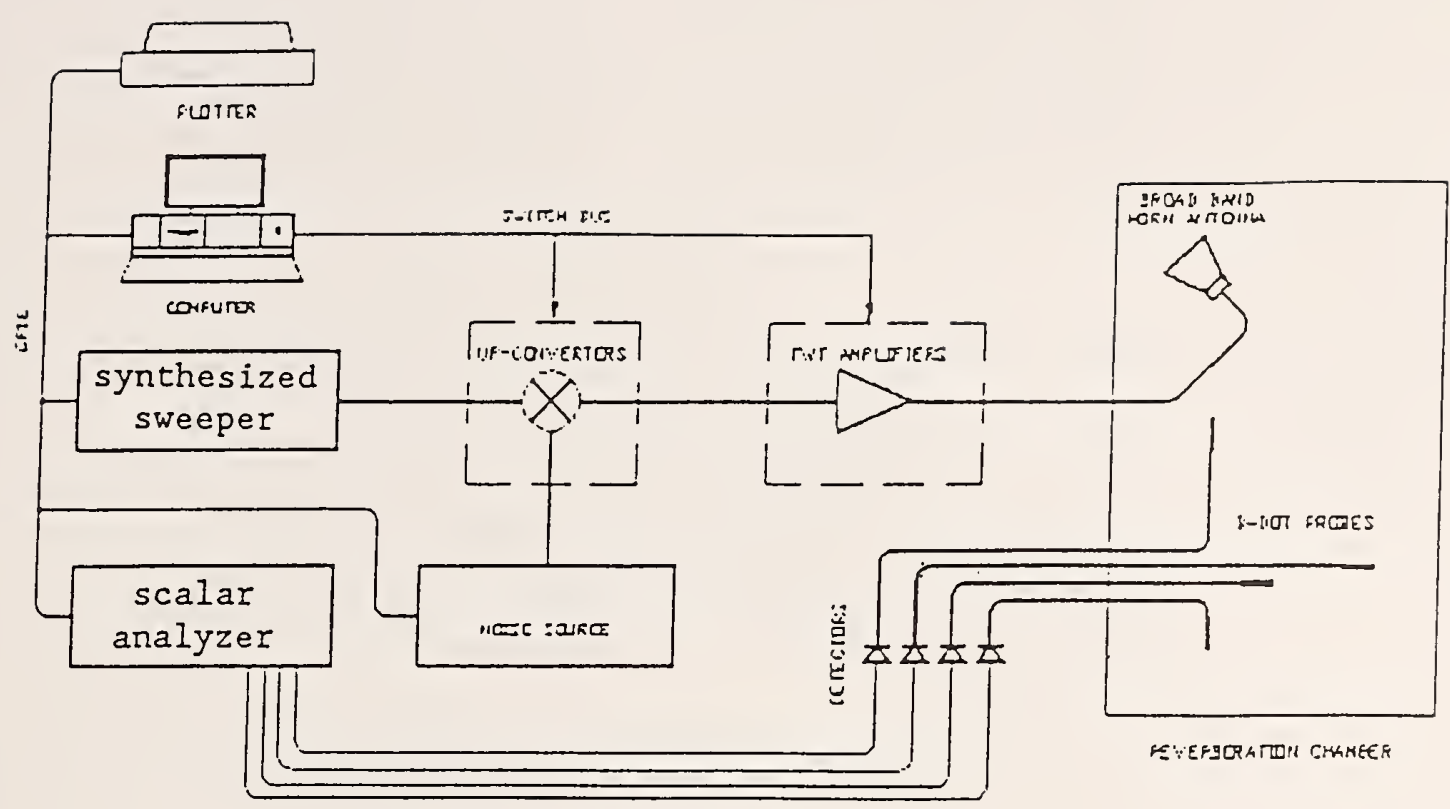

Figure 2-1. Generating and measuring NBWGN excited fields.
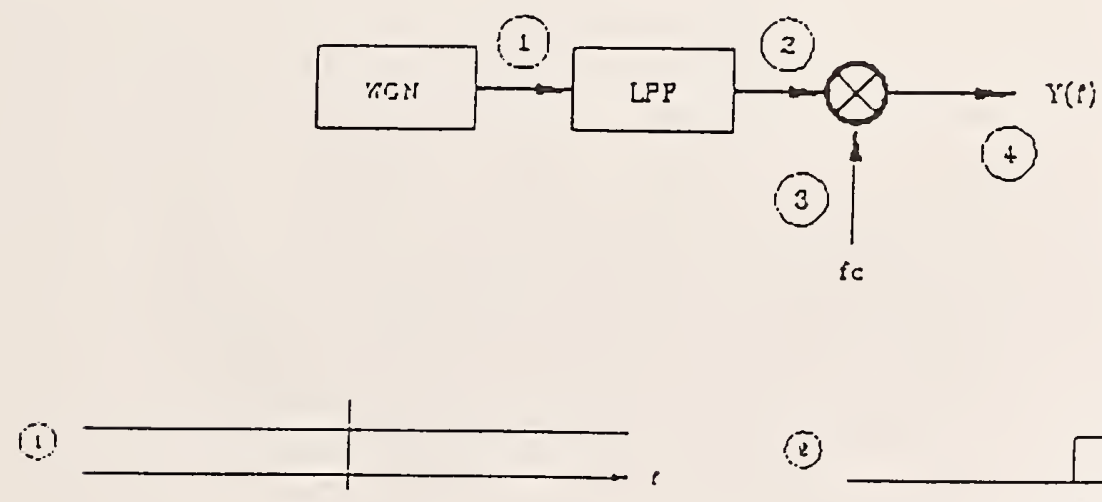

(a)
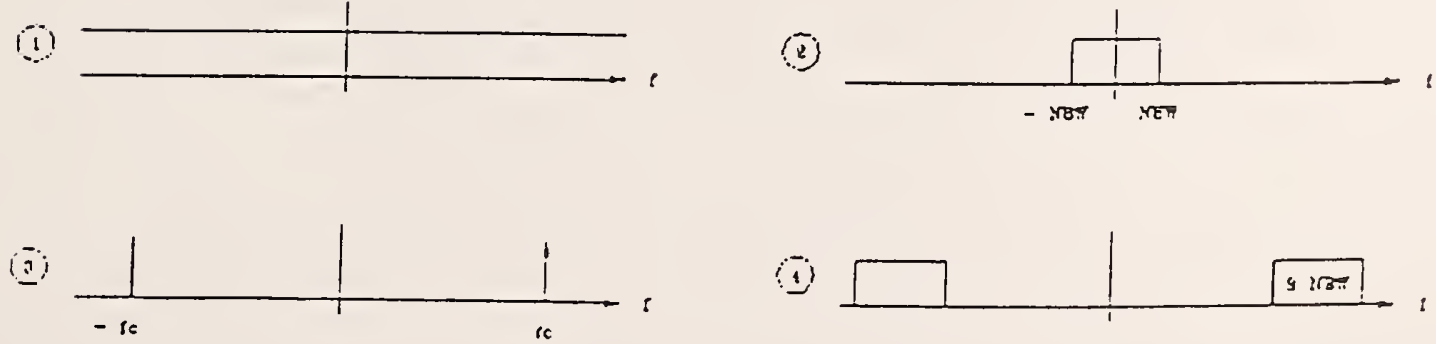

Figure 2-2. Theoretical spectrum generated by NBWGN method. 


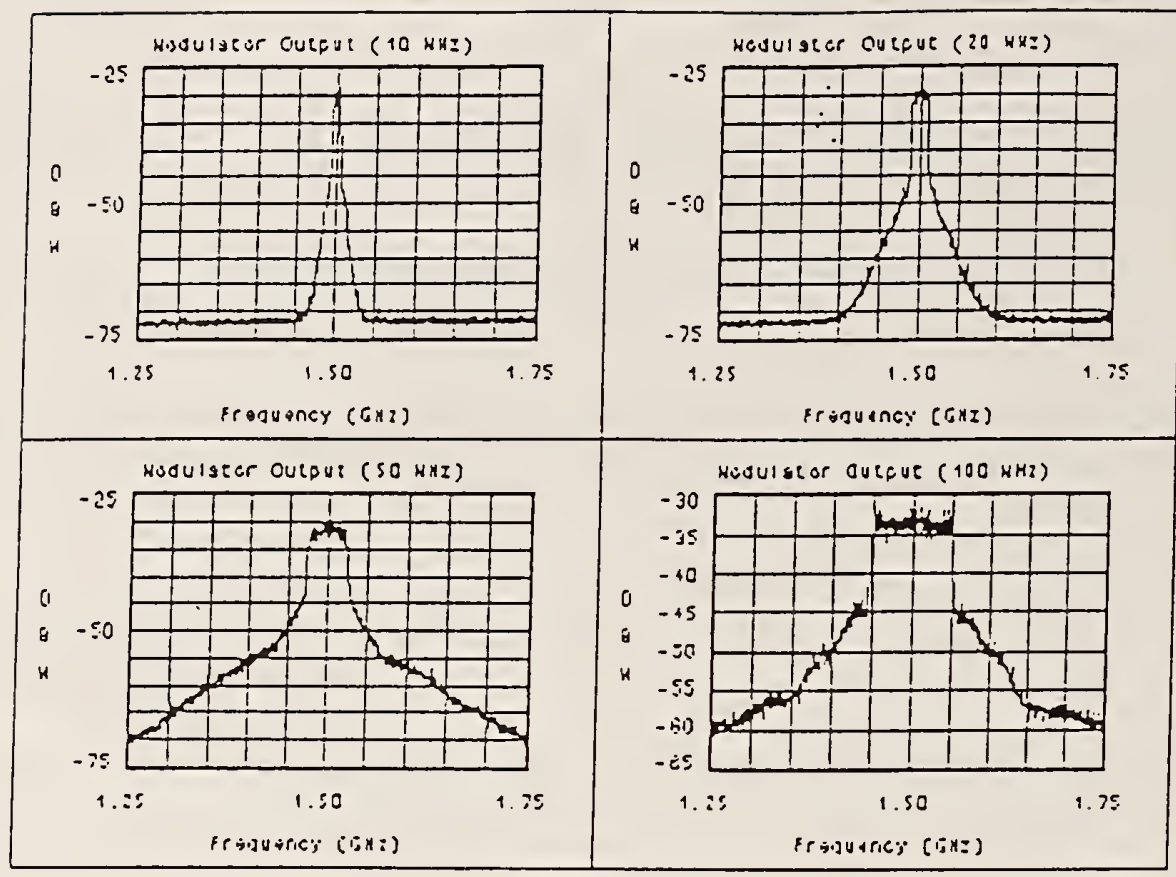

Figure 2-3. Actual output of NBWGN excitation method with excitation method with a center frequency of $1.5 \mathrm{GHz}$ and four agility bandwidths. 


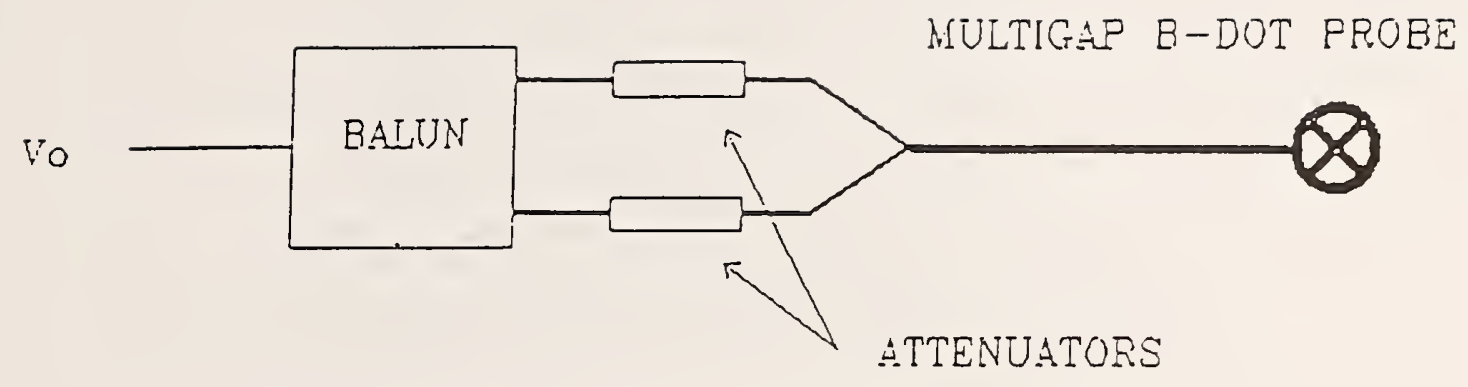

Figure 3-1. Probe-balun configuration.

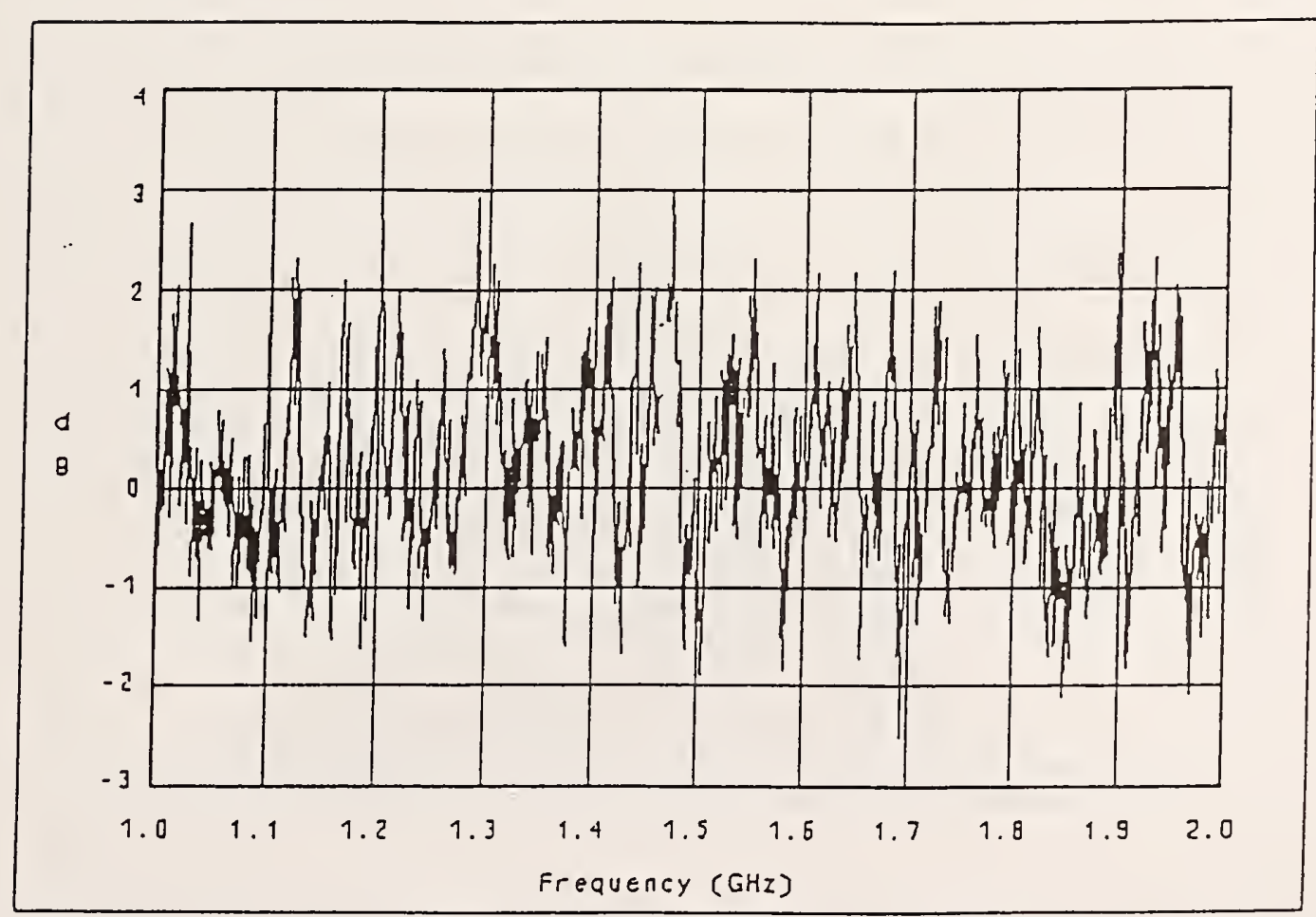

Figure 3-2. Field uniformity with $10 \mathrm{MHz}$ of NBWGN over 1 to $2 \mathrm{GHz}$. 


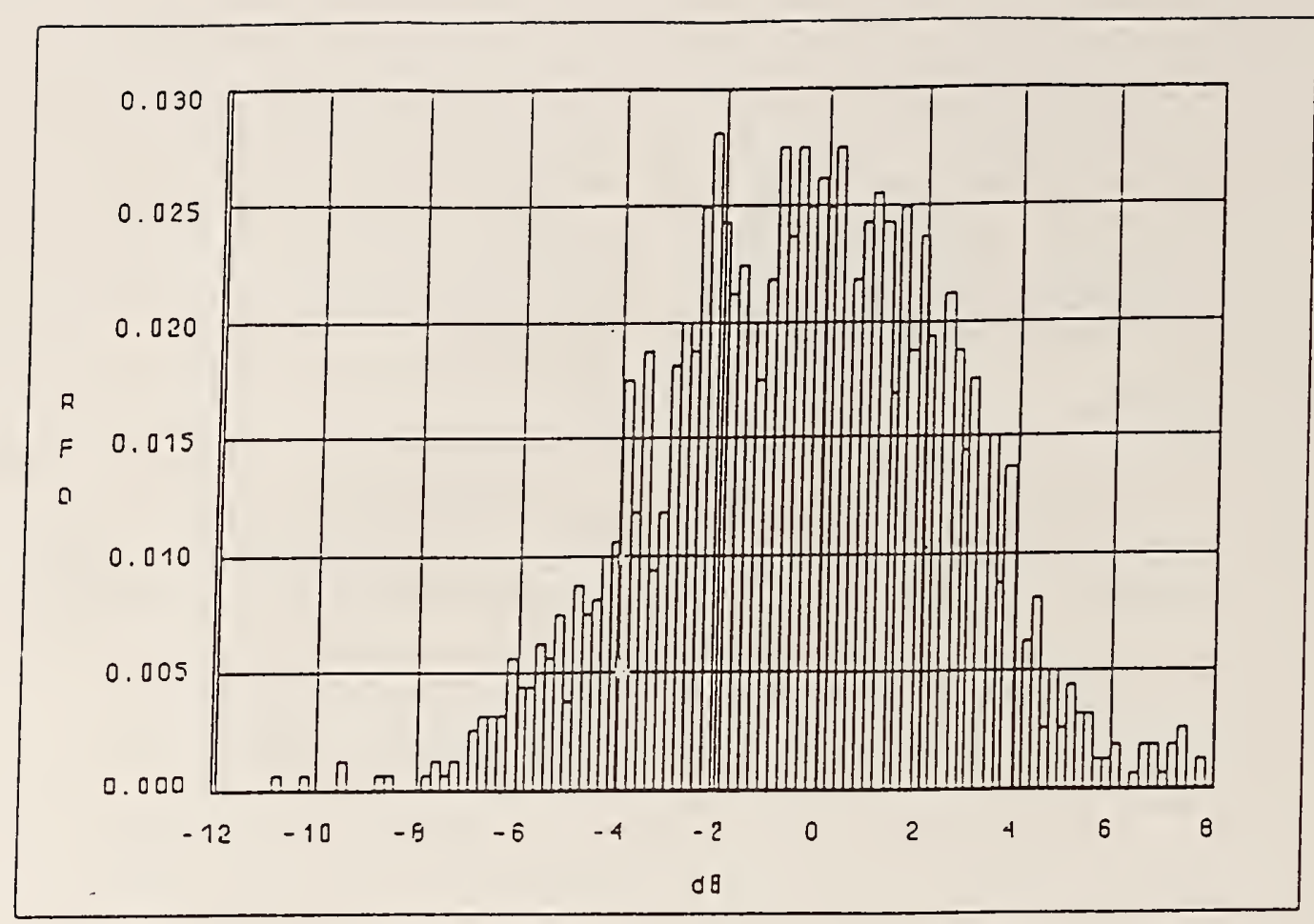

Figure 3-3. Field uniformity Relative Frequency Distribution for $2 \mathrm{MHz}$ of NBWGN at 0.5 to 1 $\mathrm{GHz}$.

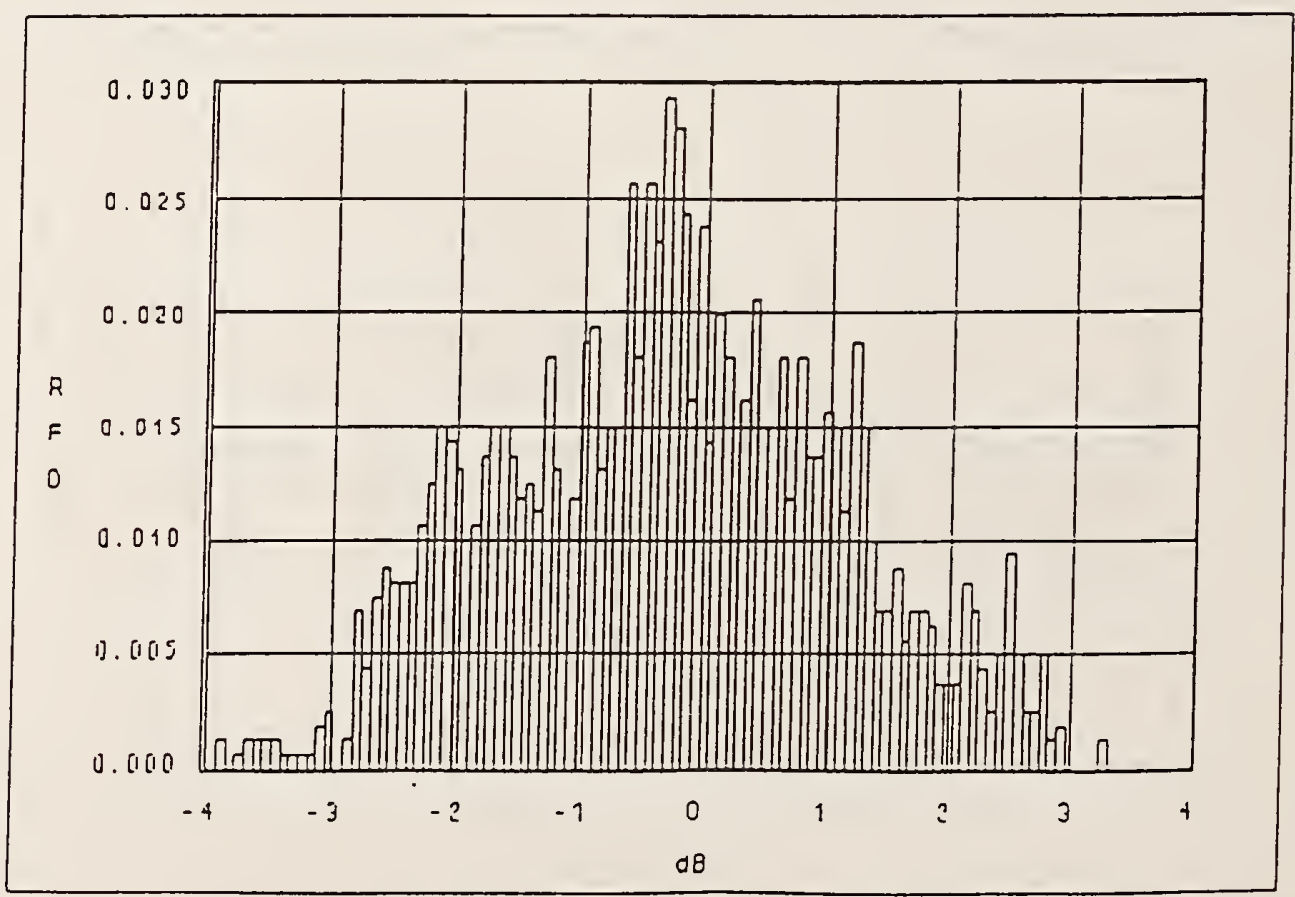

Figure 3-4. Field uniformity Relative Frequency Distribution for $10 \mathrm{MHz}$ of NBWGN at 0.5 to 1 $\mathrm{GHz}$. 


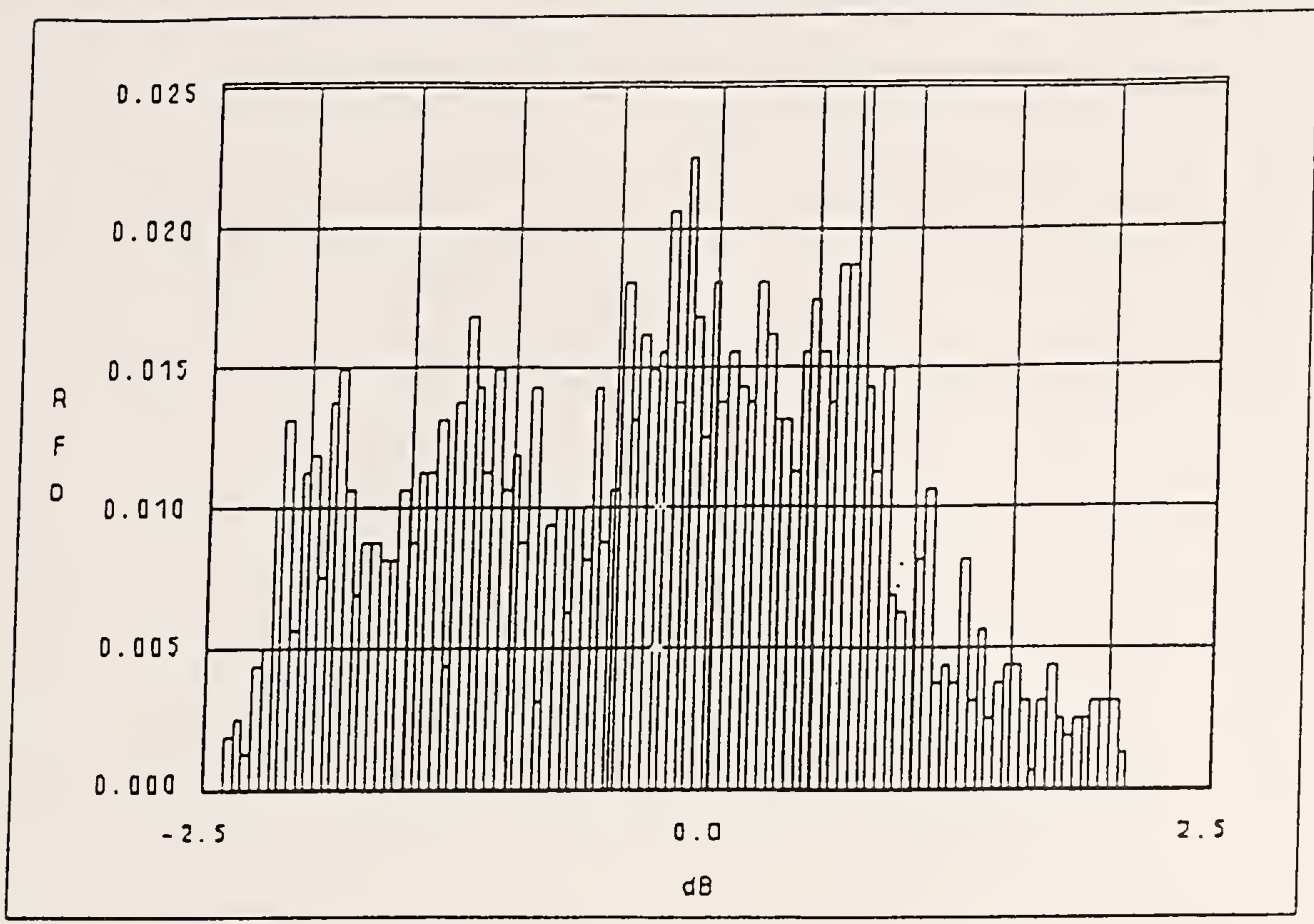

Figure 3-5. Field uniformity Relative Frequency Distribution for $20 \mathrm{MHz}$ of NBWGN at 0.5 to 1 $\mathrm{GHz}$.

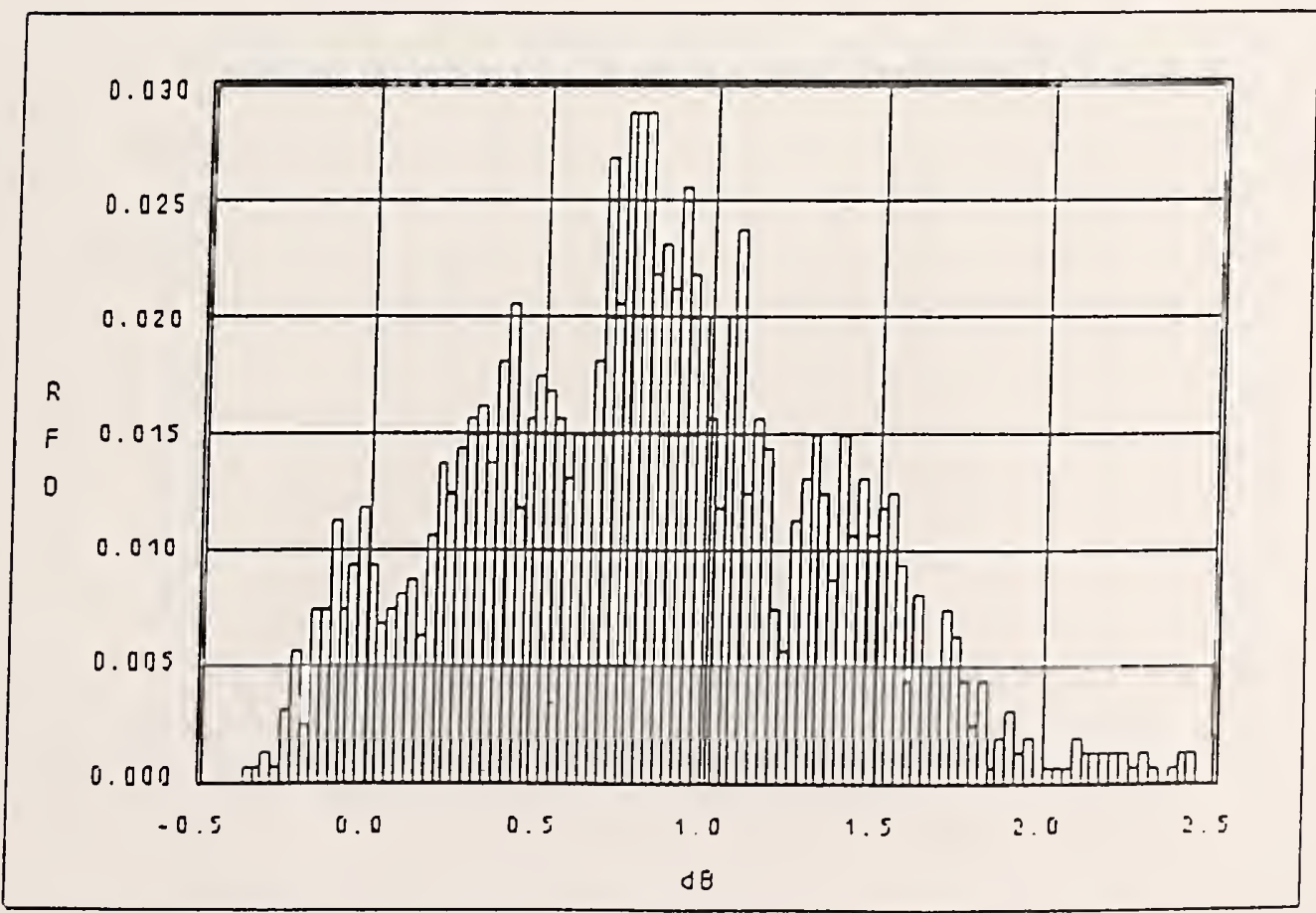

Figure 3-6. Field uniformity Relative Frequency Distribution for $50 \mathrm{MHz}$ of NBWGN at 0.5 to 1 $\mathrm{GHz}$. 


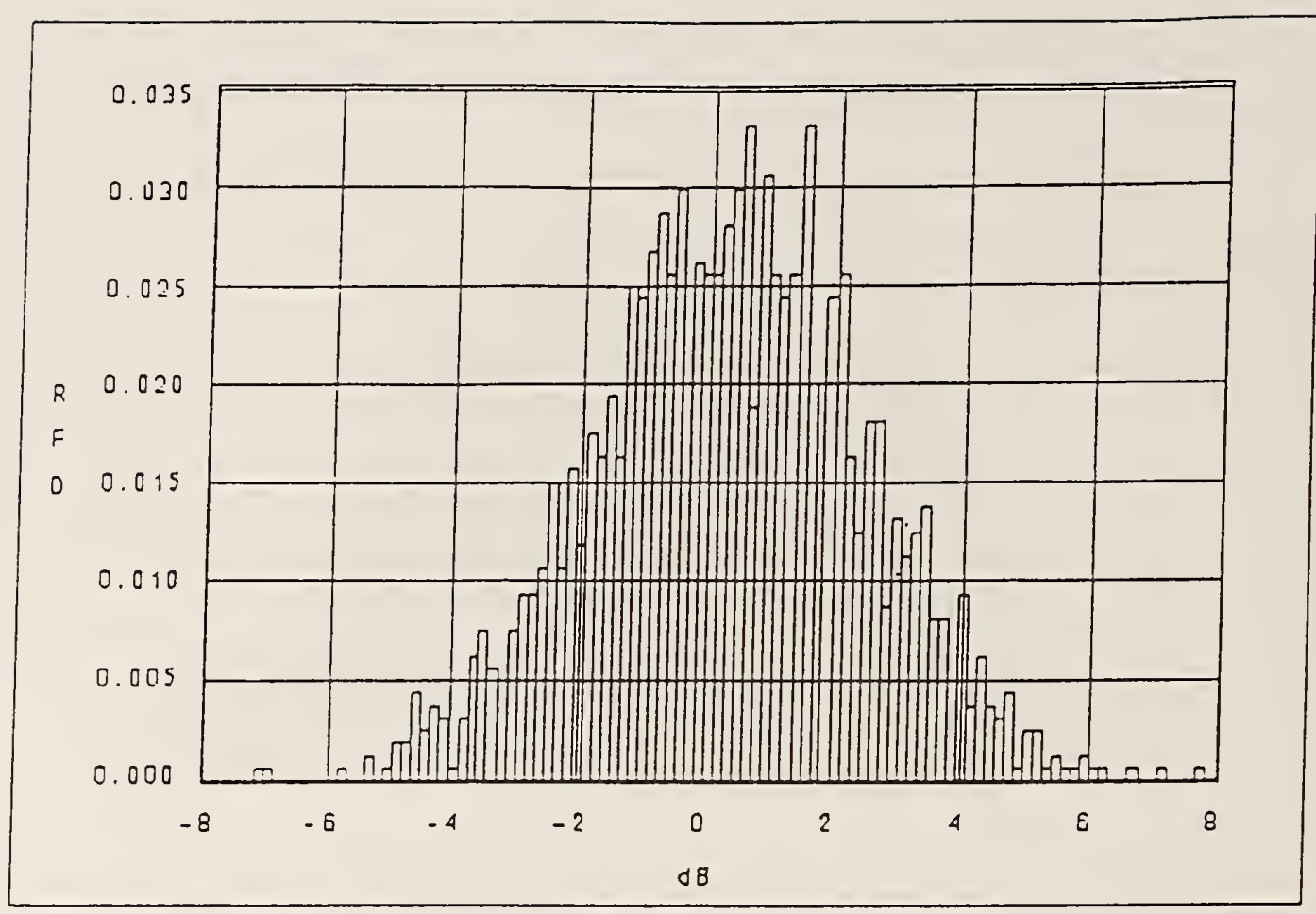

Figure 3-7. Field uniformity Relative Frequency Distribution for $2 \mathrm{MHz}$ of NBWGN at 1 to $2 \mathrm{GHz}$.

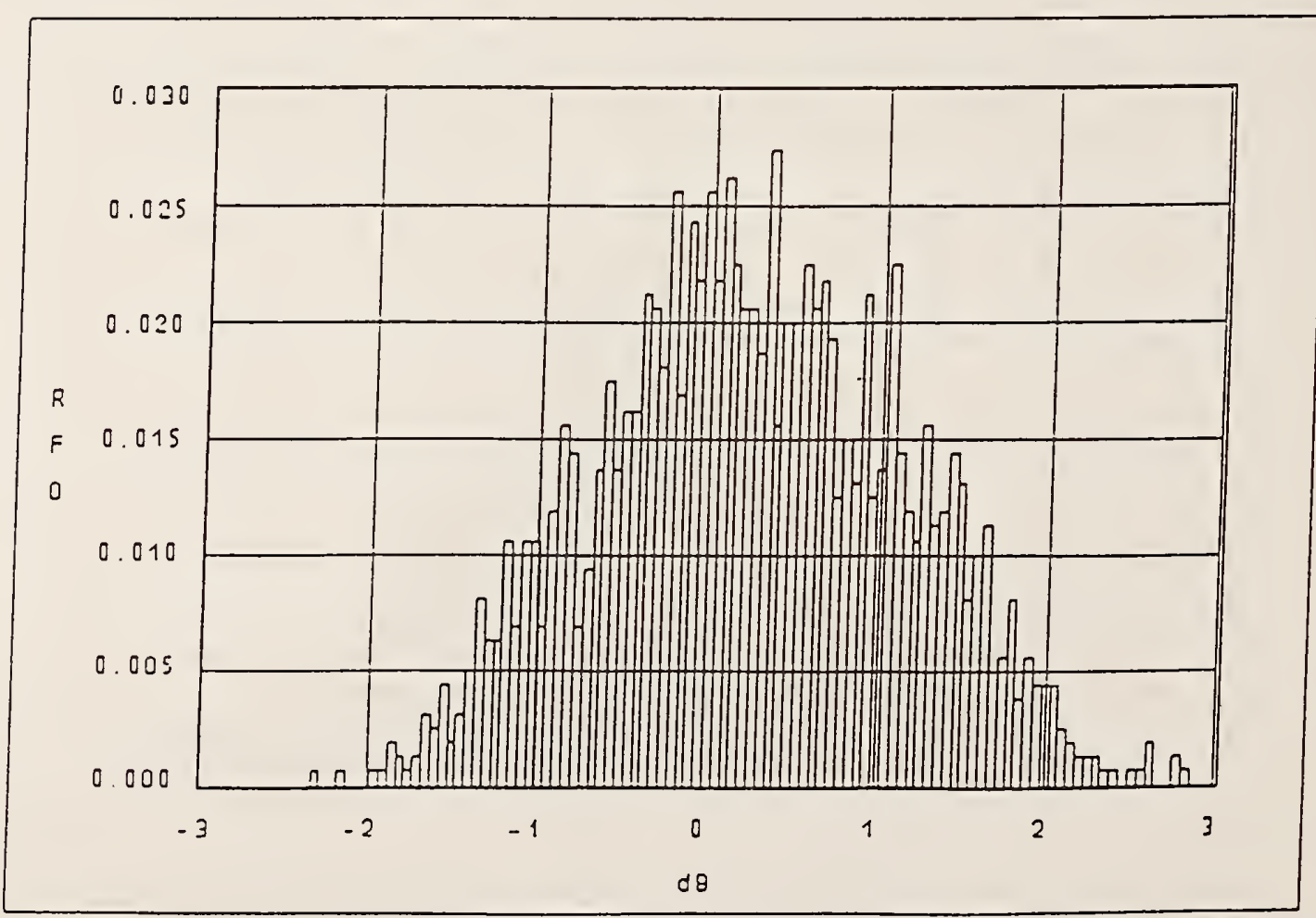

Figure 3-8. Field uniformity Relative Frequency Distribution for $10 \mathrm{MHz}$ of NBWGN at 1 to $2 \mathrm{GHz}$. 


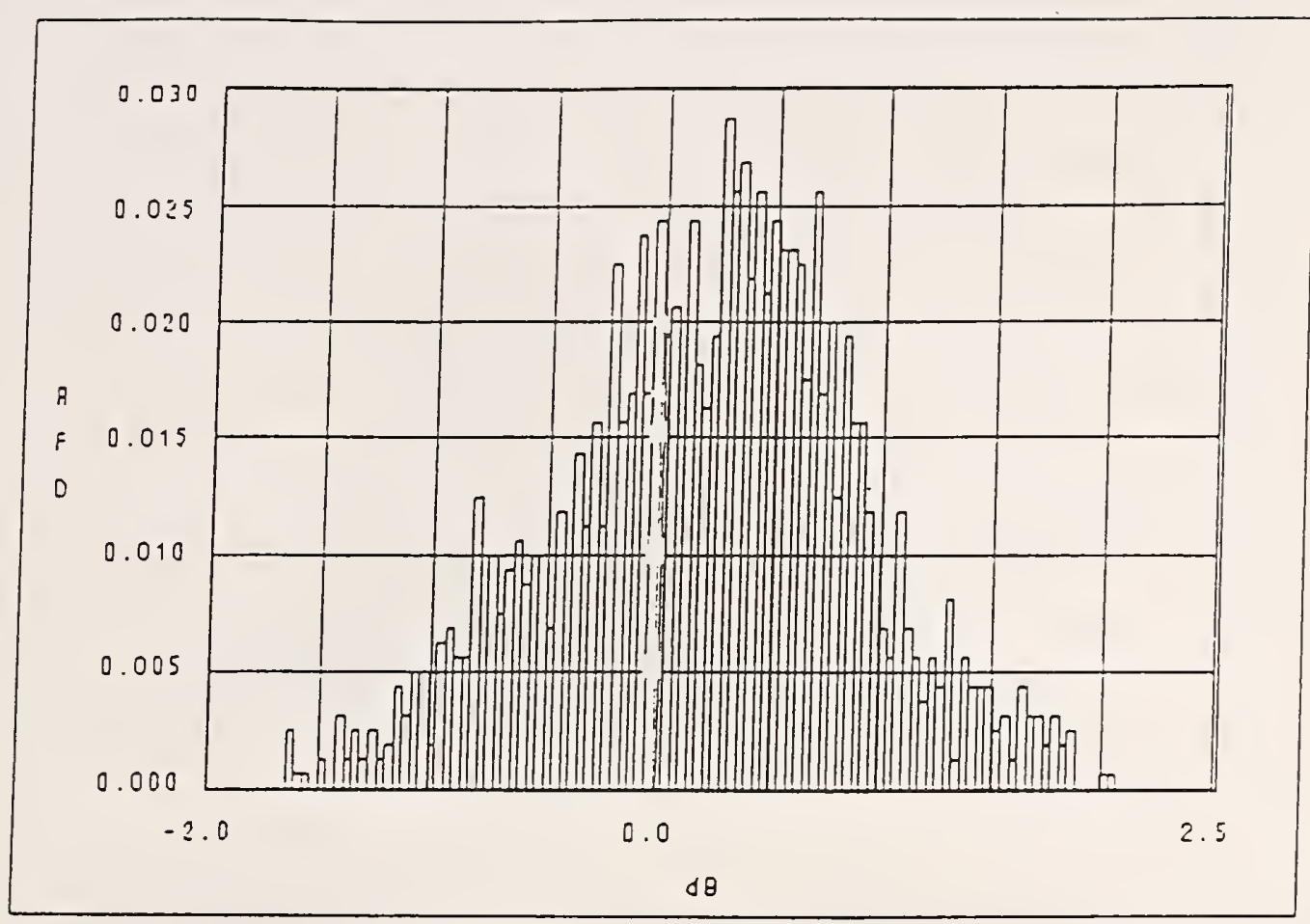

Figure 3-9. Field uniformity Relative Frequency Distribution for $20 \mathrm{MHz}$ of NBWGN at 1 to $2 \mathrm{GHz}$.

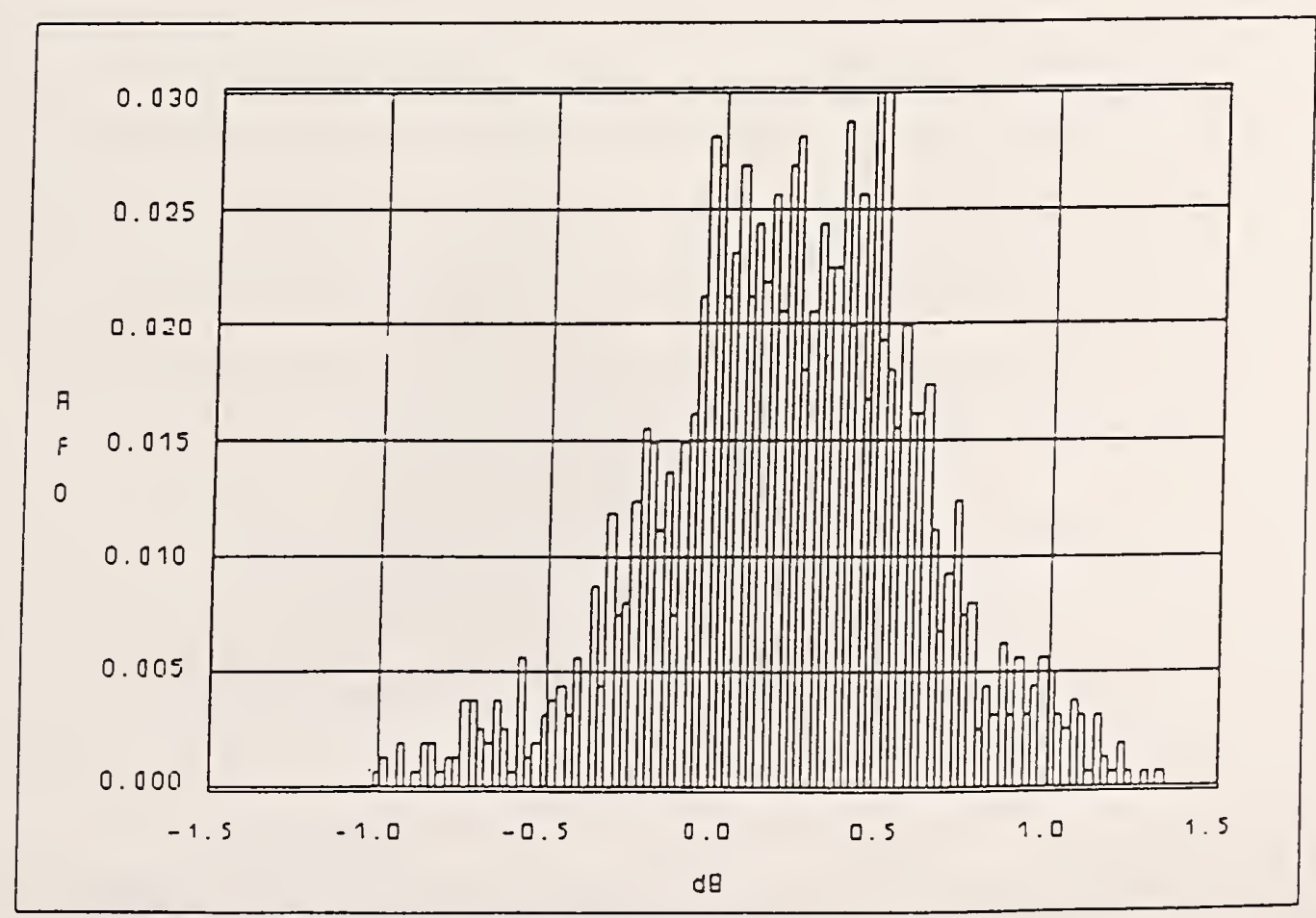

Figure 3-10. Field uniformity Relative Frequency Distribution for $50 \mathrm{MHz}$ of NBWGN at 1 to $2 \mathrm{GHz}$. 


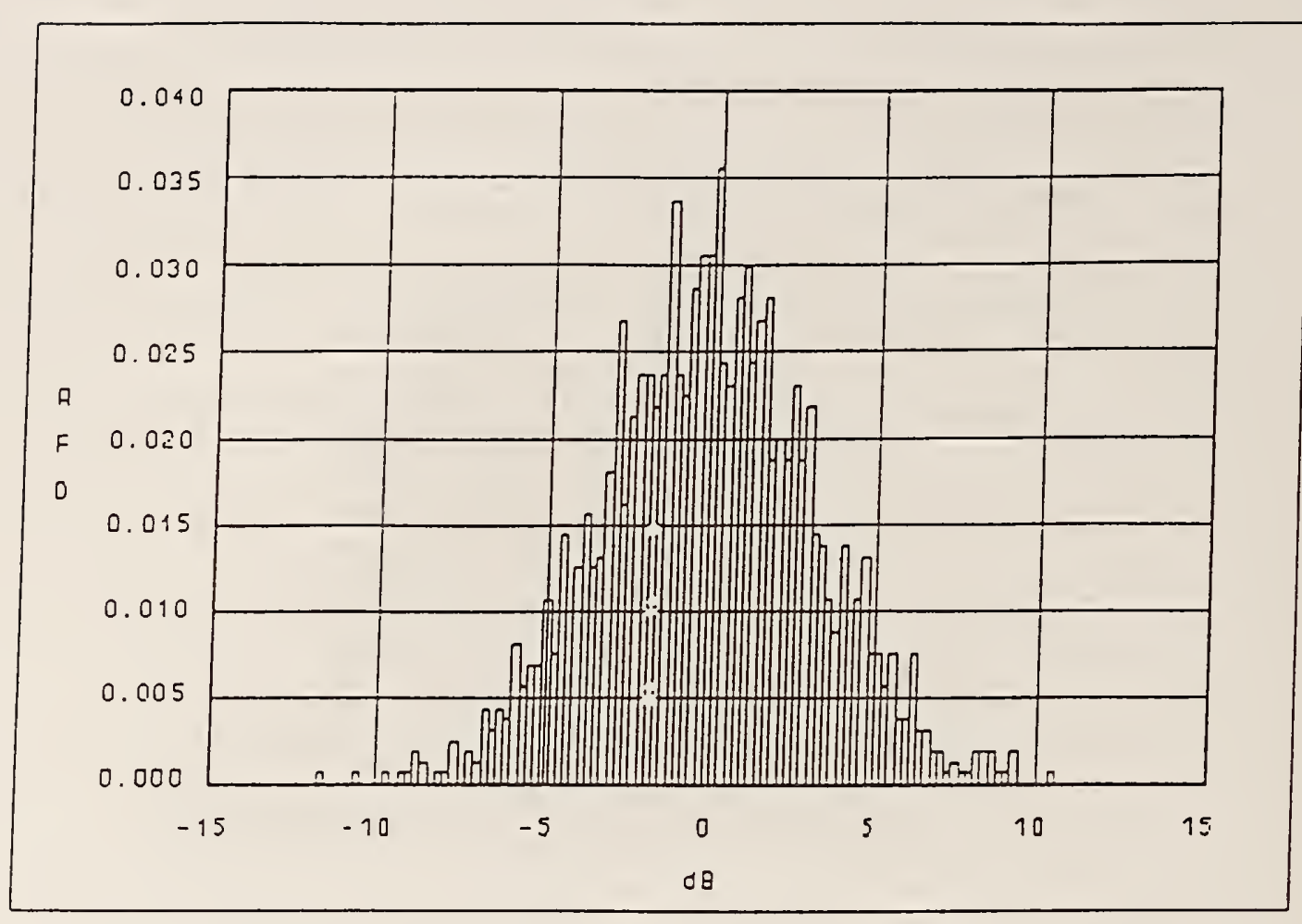

Figure 3-11. Field uniformity Relative Frequency Distribution for $2 \mathrm{MHz}$ of NBWGN at 2 to $4 \mathrm{GHz}$.

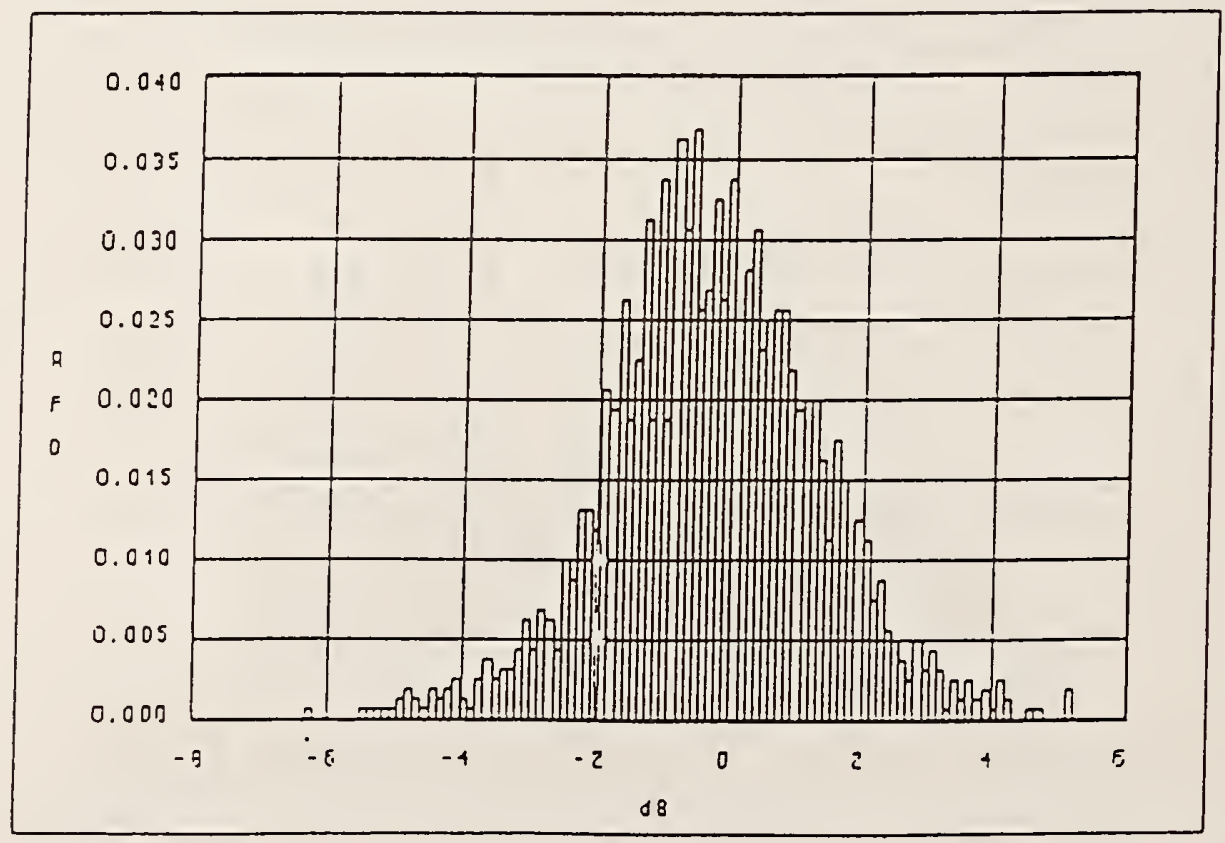

Figure 3-12. Field uniformity Relative Frequency Distribution for $10 \mathrm{MHz}$ of NBWGN at 2 to $4 \mathrm{GHz}$. 


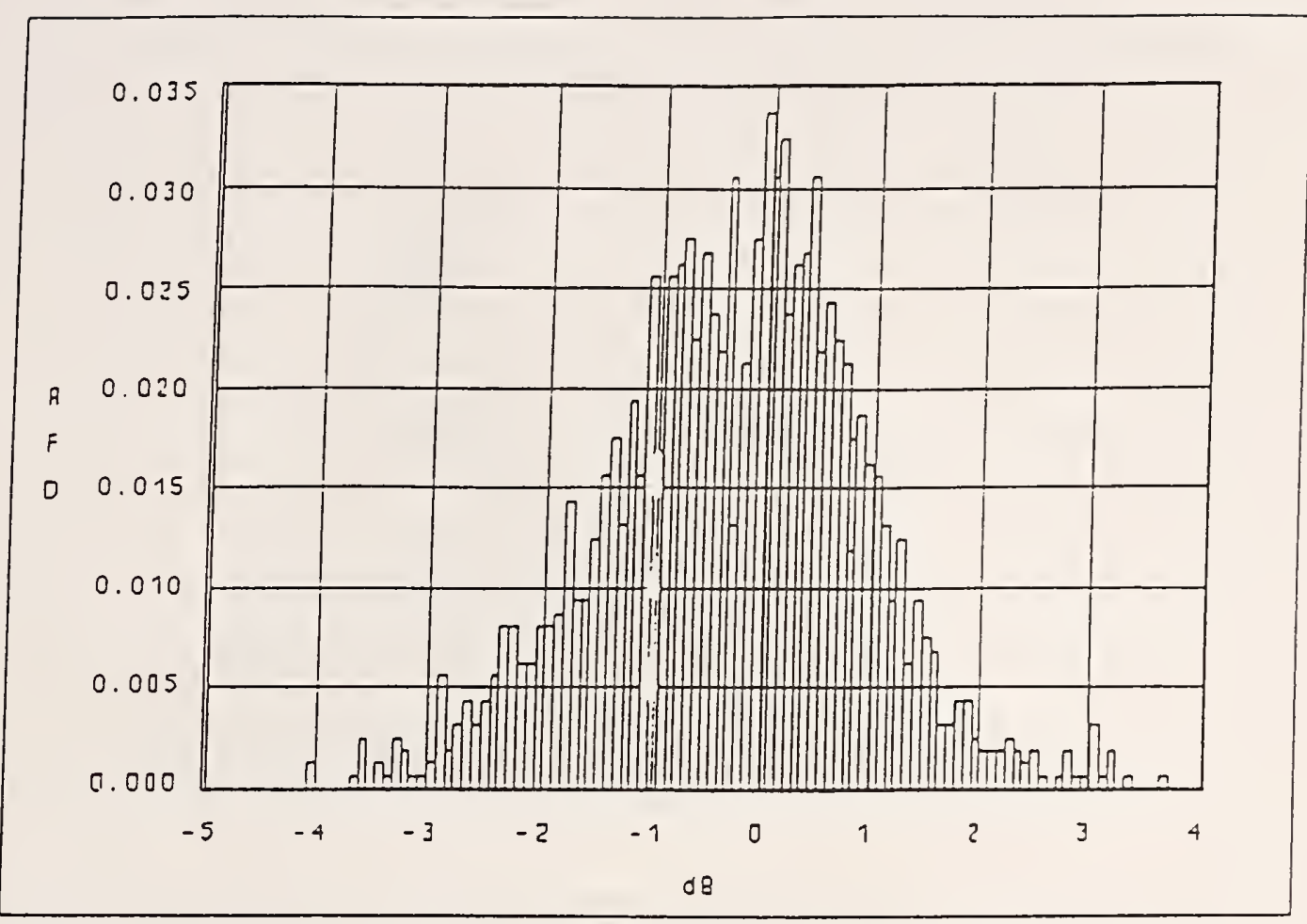

Figure 3-13. Field uniformity Relative Frequency Distribution for $20 \mathrm{MHz}$ of NBWGN at 2 to $4 \mathrm{GHz}$.

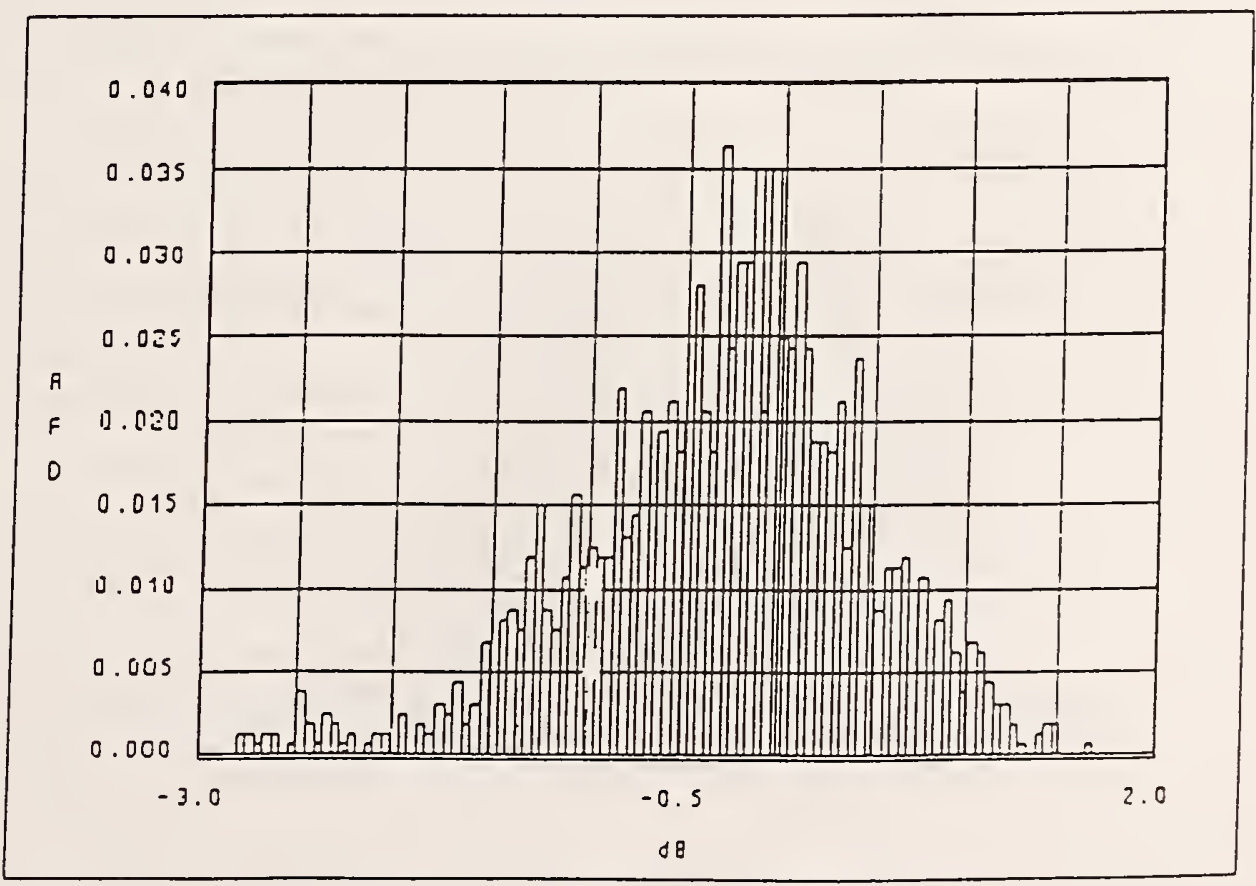

Figure 3-14. Field uniformity Relative Frequency Distribution for $50 \mathrm{MHz}$ of NBWGN at 2 to $4 \mathrm{GHz}$. 


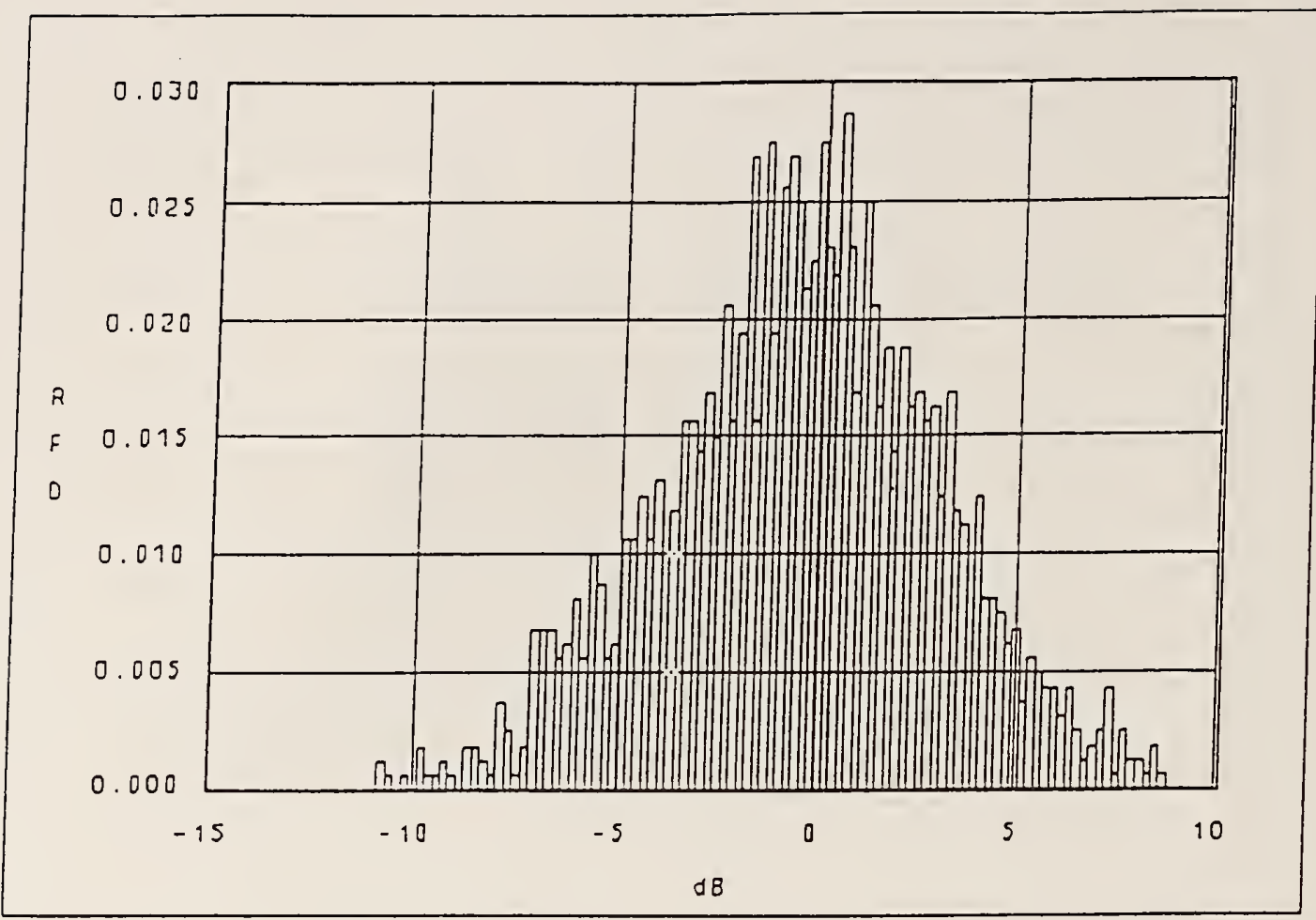

Figure 3-15. Field uniformity Relative Frequency Distribution for $2 \mathrm{MHz}$ of NBWGN at 4 to $8 \mathrm{GHz}$.

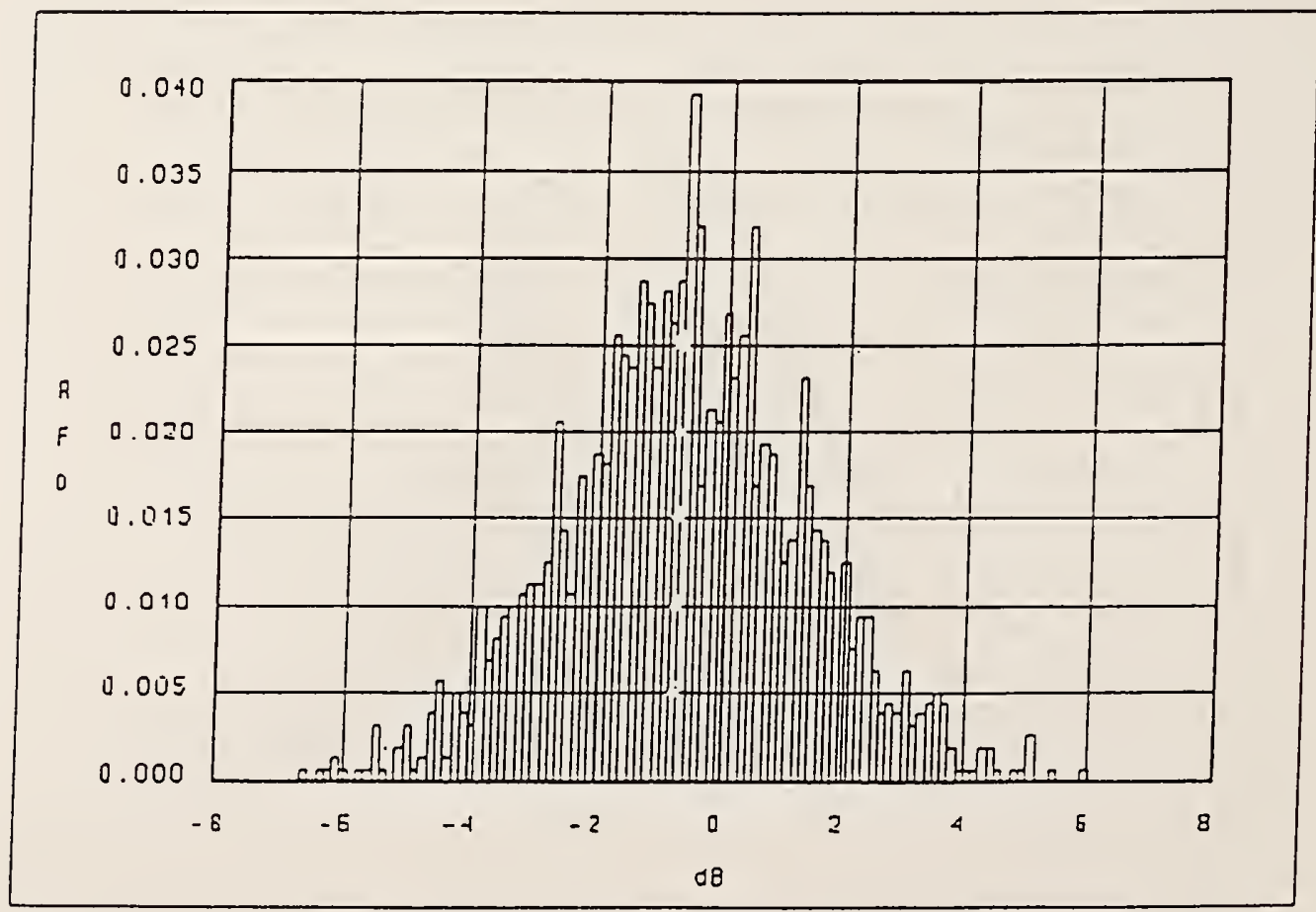

Figure 3-16. Field uniformity Relative Frequency Distribution for $10 \mathrm{MHz}$ of NBWGN at 4 to $8 \mathrm{GHz}$. 


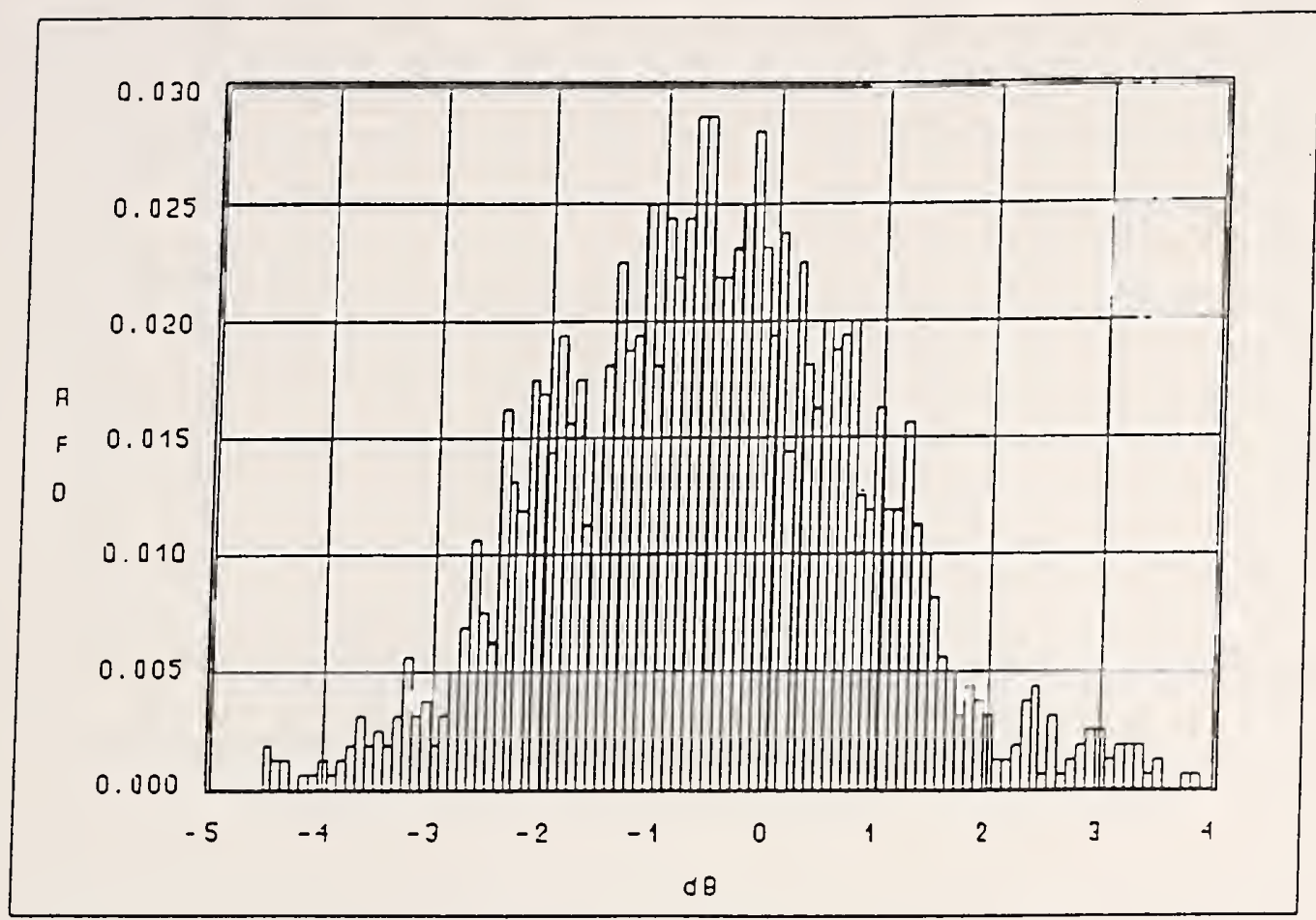

Figure 3-17. Field uniformity Relative Frequency Distribution for $20 \mathrm{MHz}$ of NBWGN at 4 to $8 \mathrm{GHz}$.

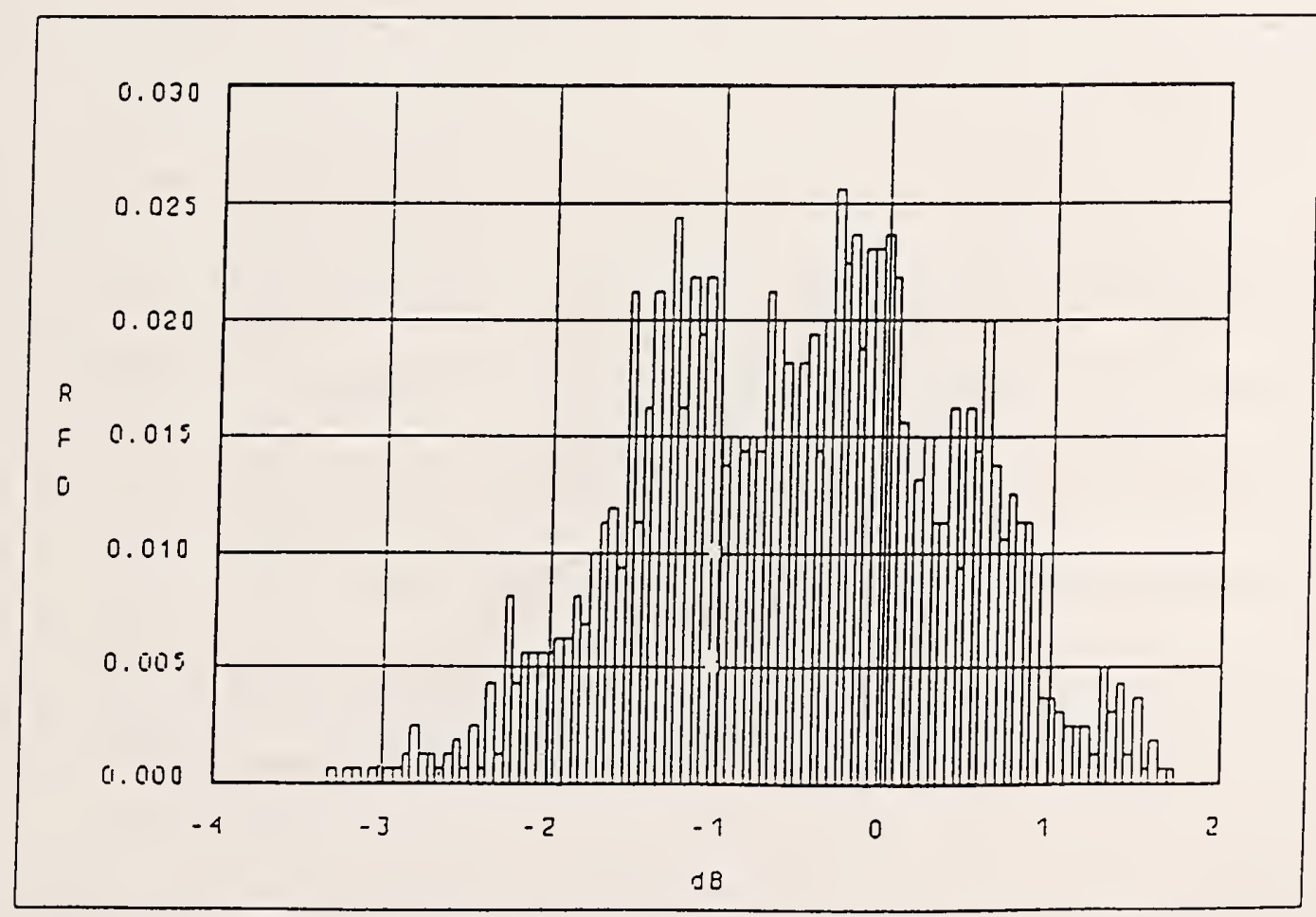

Figure 3-18. Field uniformity Relative Frequency Distribution for $50 \mathrm{MHz}$ of NBWGN at 4 to $8 \mathrm{GHz}$. 


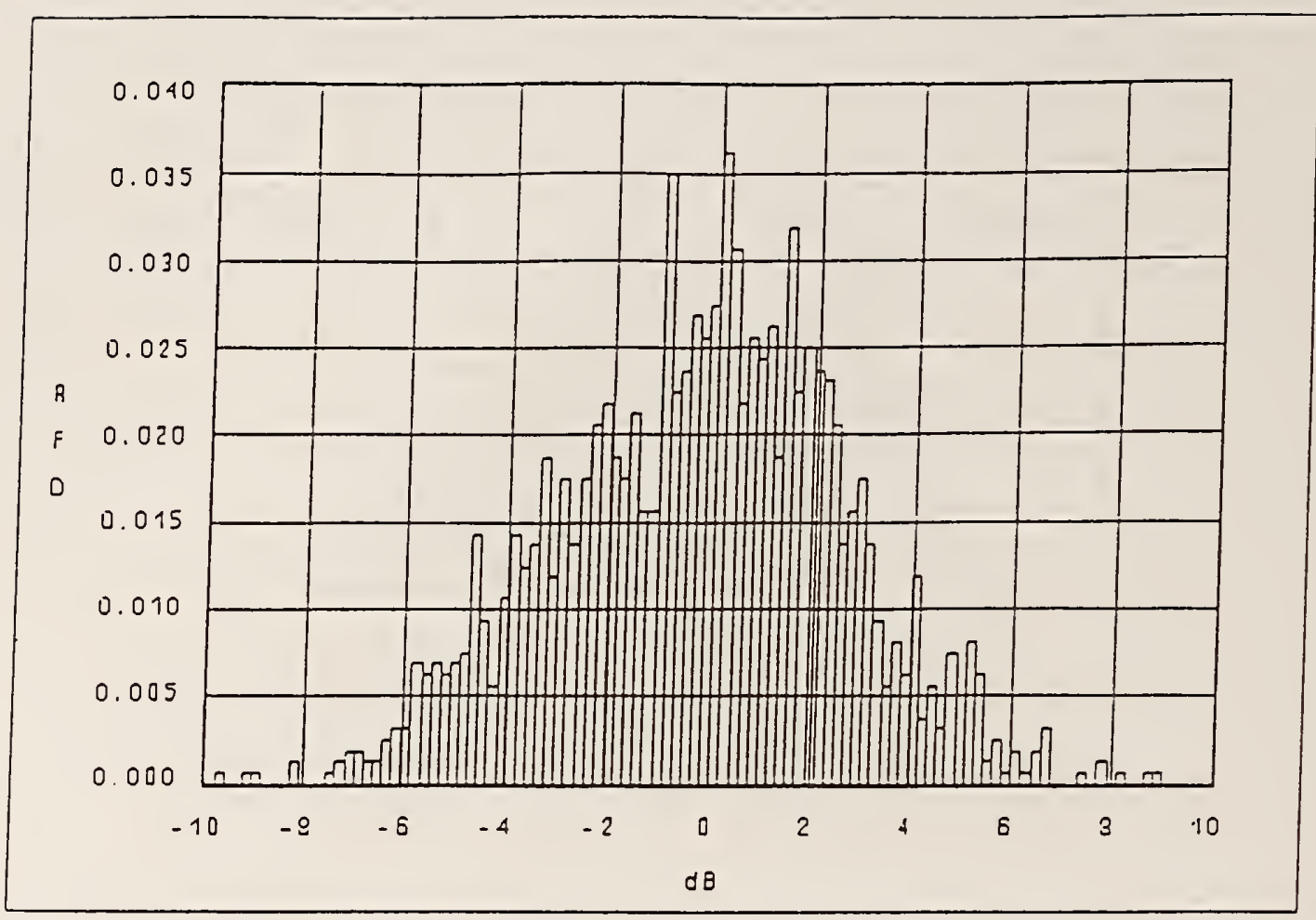

Figure 3-19. Field uniformity Relative Frequency Distribution for $2 \mathrm{MHz}$ of NBWGN at 8 to $18 \mathrm{GHz}$.

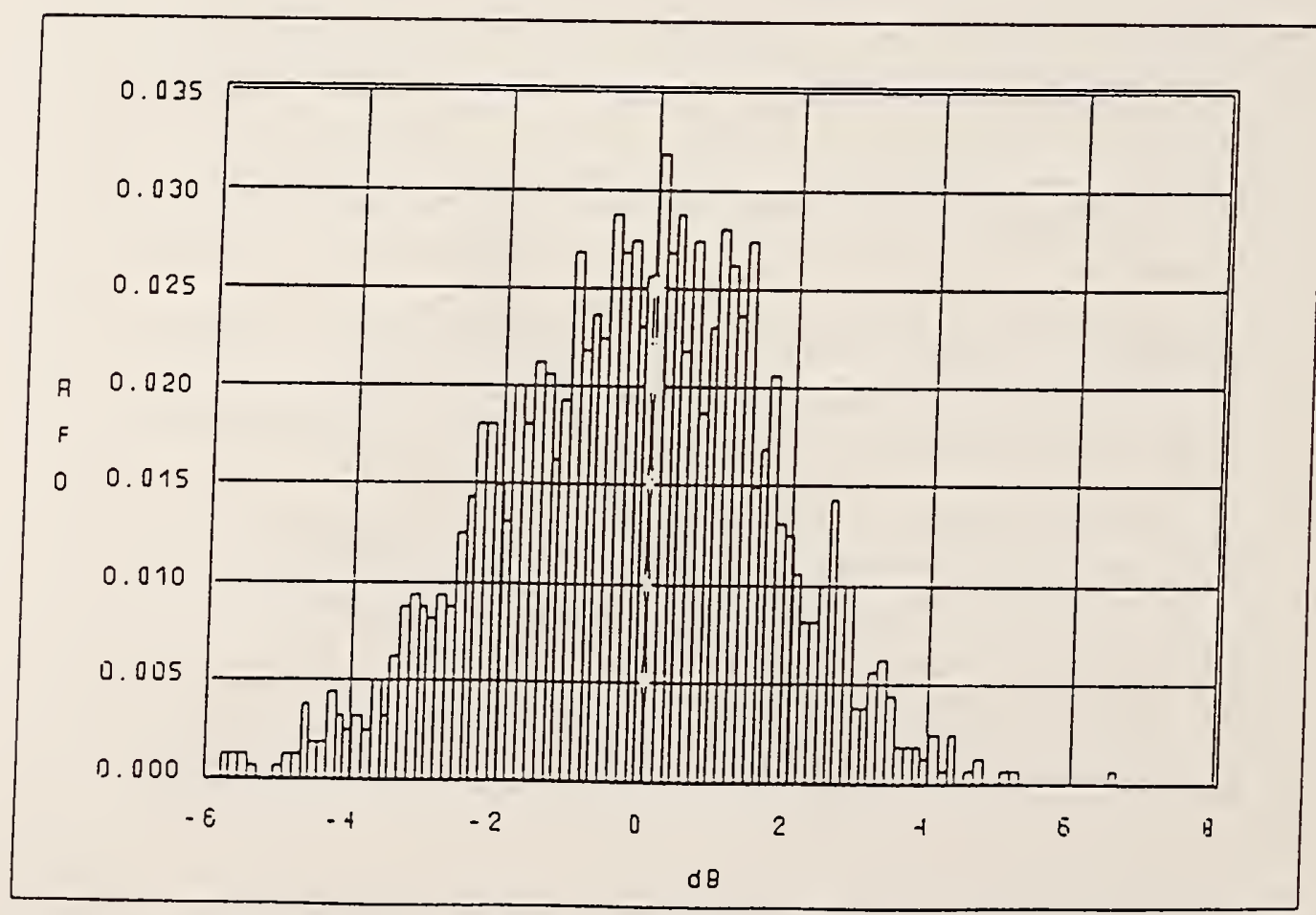

Figure 3-20. Field uniformity Relative Frequency Distribution for $10 \mathrm{MHz}$ of NBWGN at 8 to 18 $\mathrm{GHz}$. 


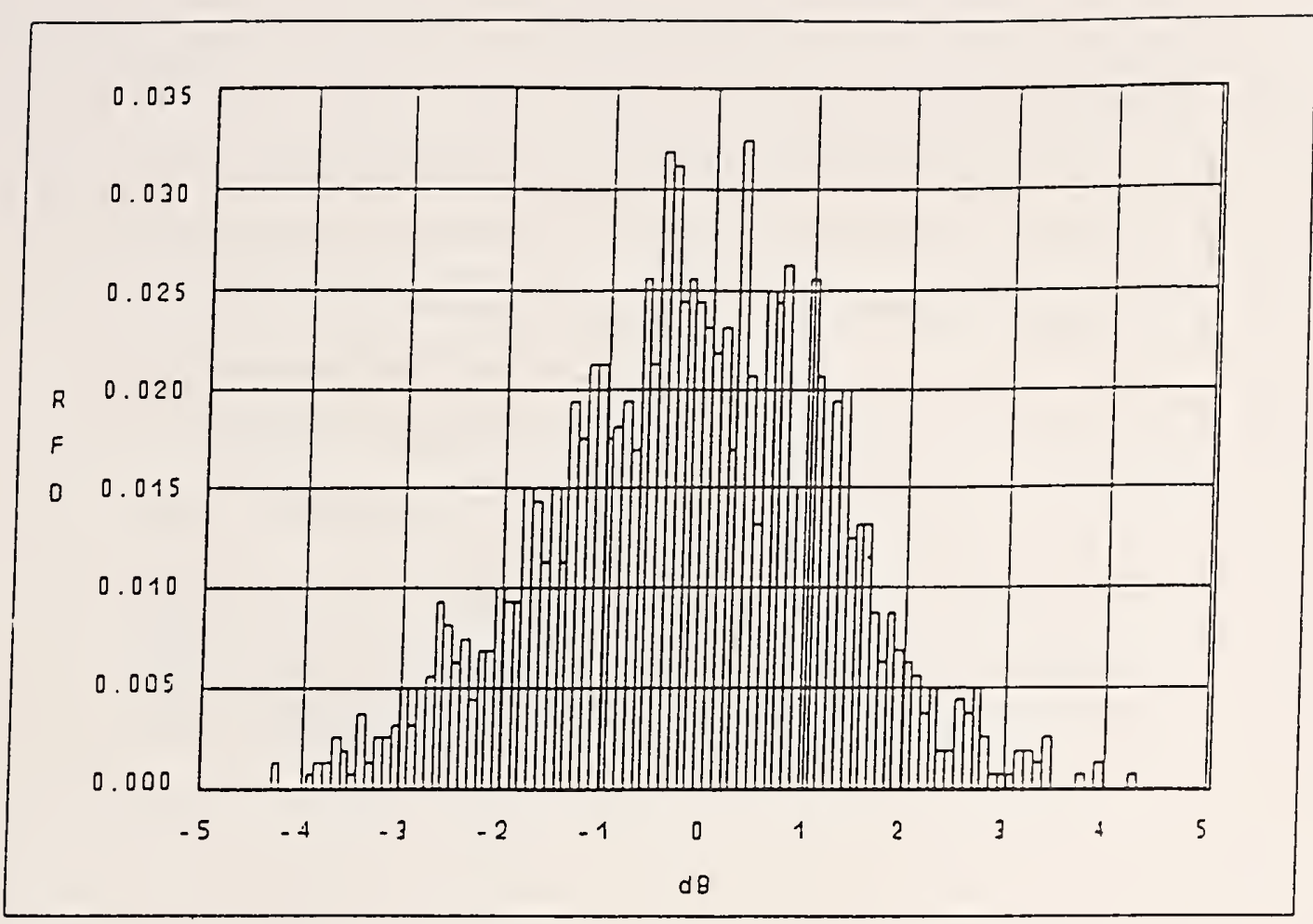

Figure 3-21. Field uniformity Relative Frequency Distribution for $20 \mathrm{MHz}$ of NBWGN at 8 to 18 $\mathrm{GHz}$.

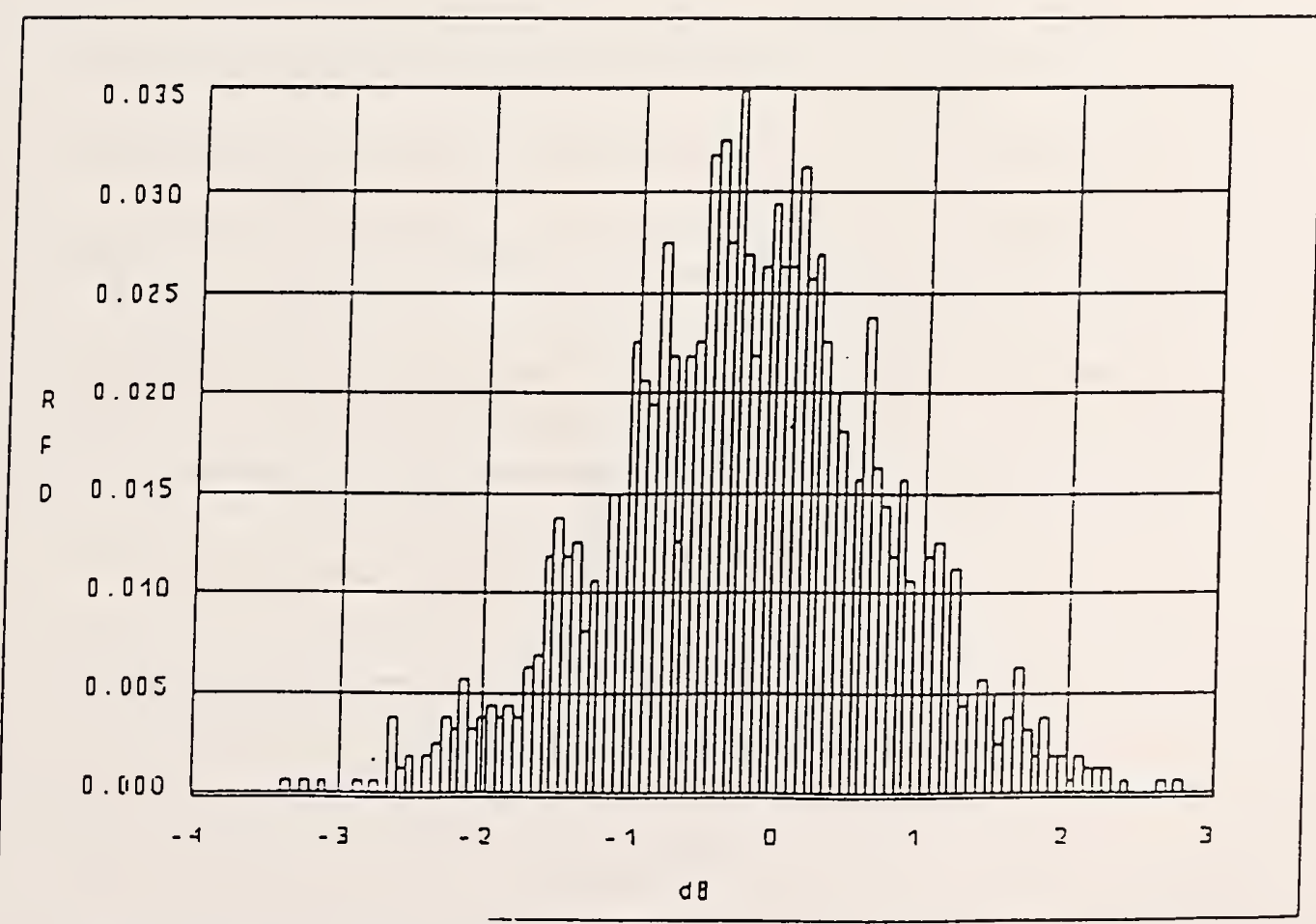

Figure 3-22. Field uniformity Relative Frequency Distribution for $50 \mathrm{MHz}$ of NBWGN at 8 to 18 $\mathrm{GHz}$. 


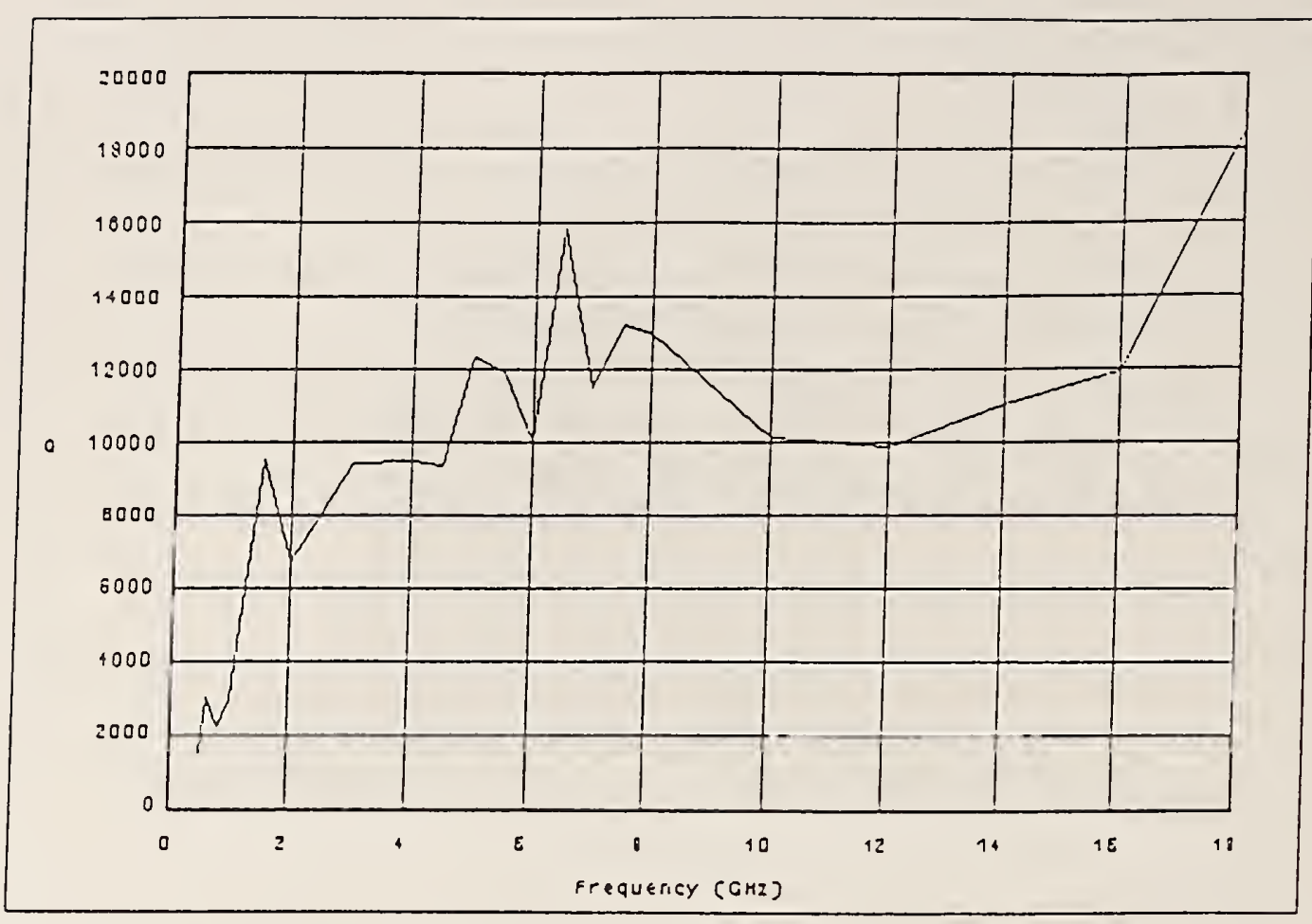

Figure 3-23. Average measured $Q$ for the NIST chamber.

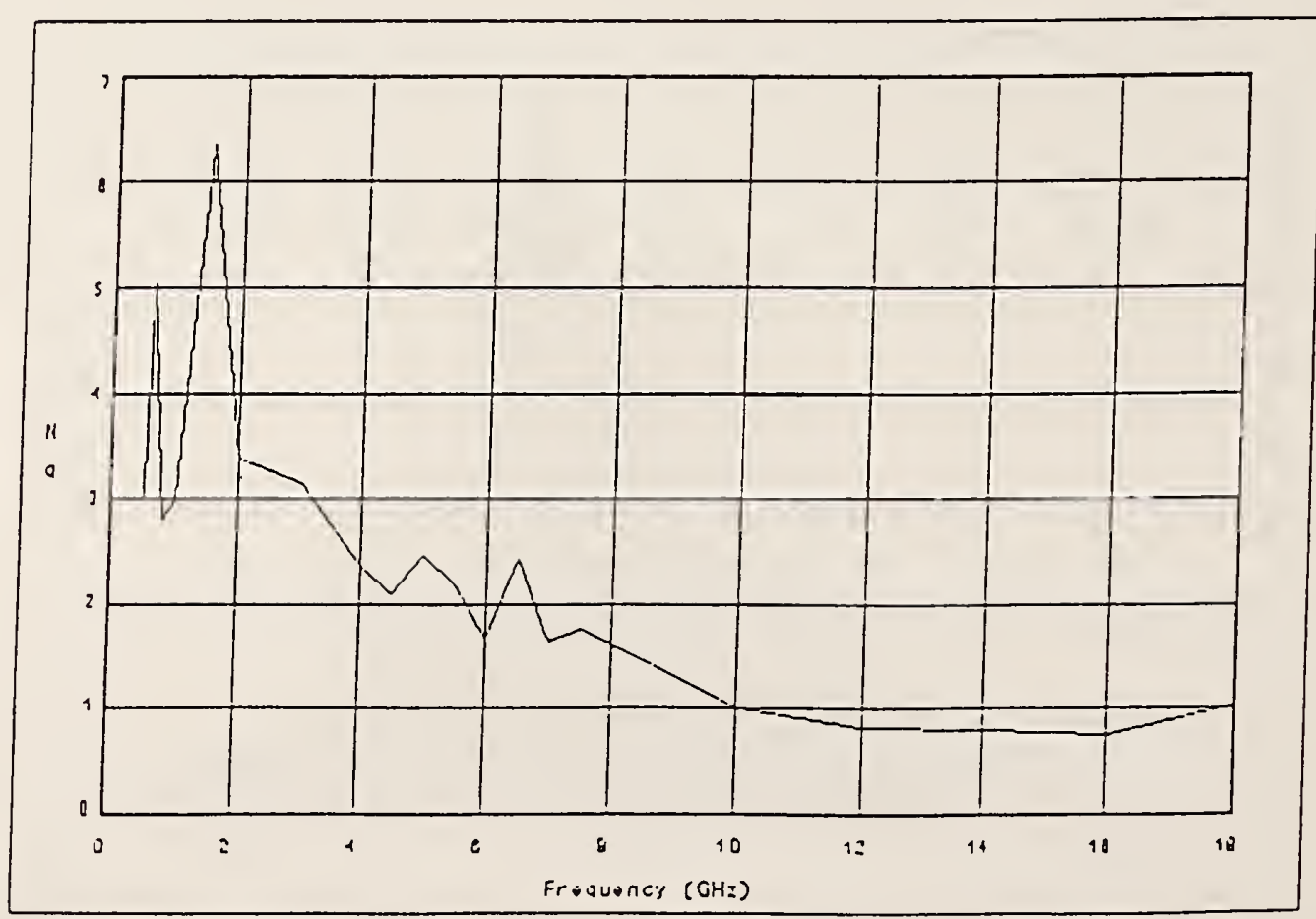

Figure 3-24. Equivalent modes per megahertz of NBWGN available for mode mixing. 


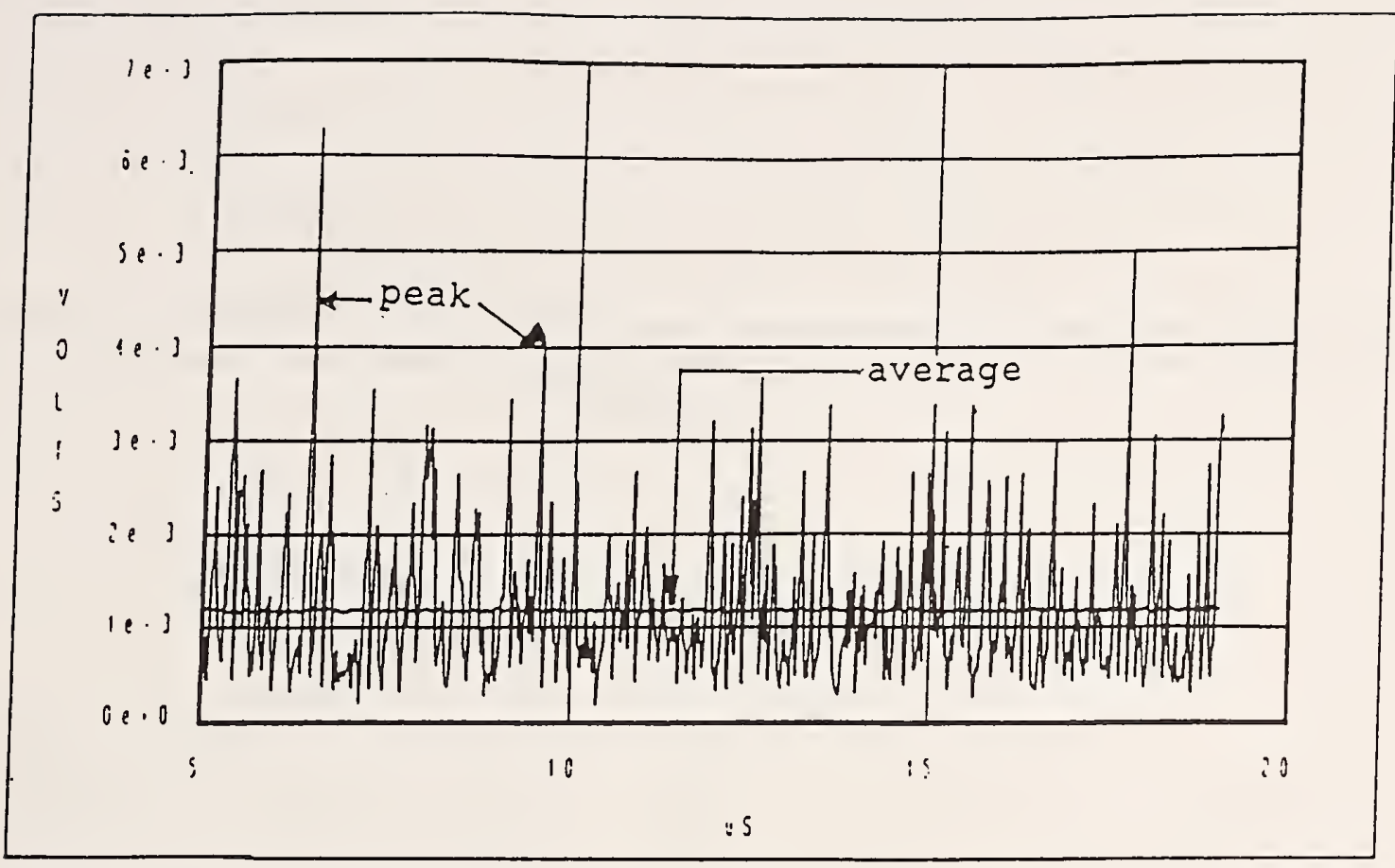

Figure 3-25. Chamber power density peak-to-average ratio measurement for NBWGN excitation.

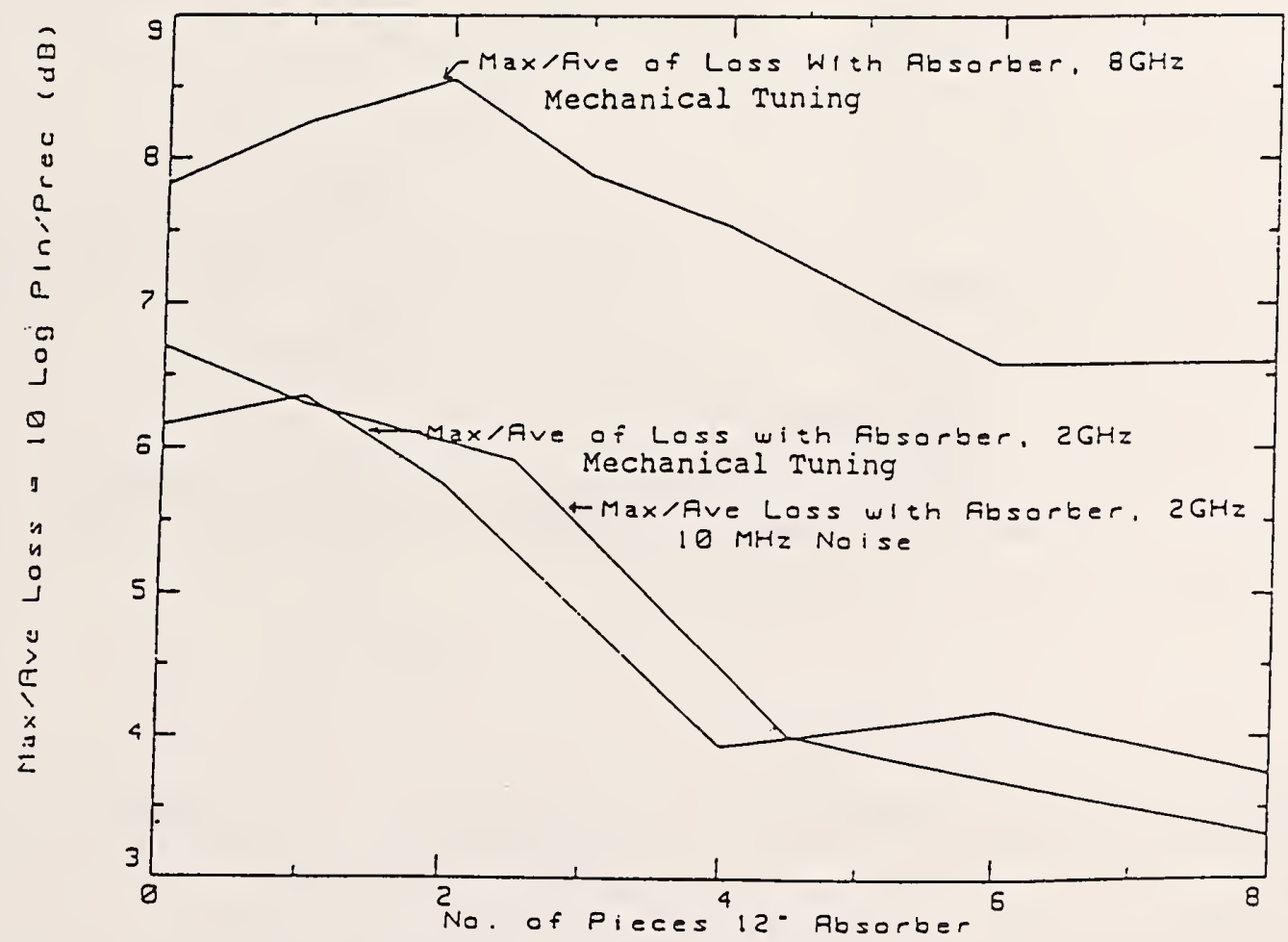

Figure 3-26. Chamber power density peak-to-average ratio measurement for $\mathrm{CW}$ (mechanical tuning) and $10 \mathrm{MHz}$ BW NBWGN excitation as a function of absorber loading (Q). 


\section{SHIELDED ENCLOSURE}

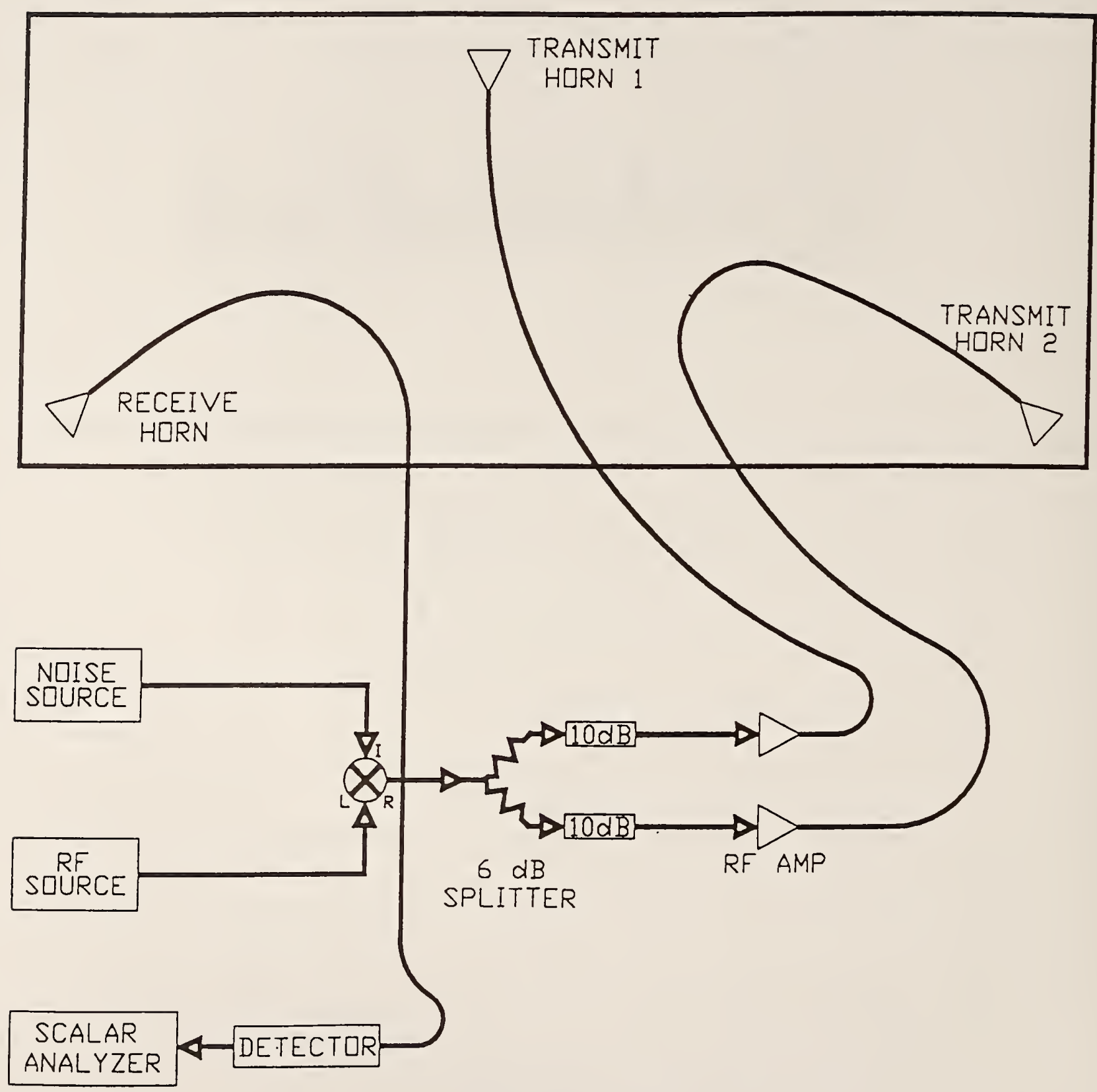

Figure 3-27. Block diagram of system for increasing test field inside reverberation chamber using a single noise source and mixer and two rf amplifiers. 


\section{SHIELDED ENCLISURE}

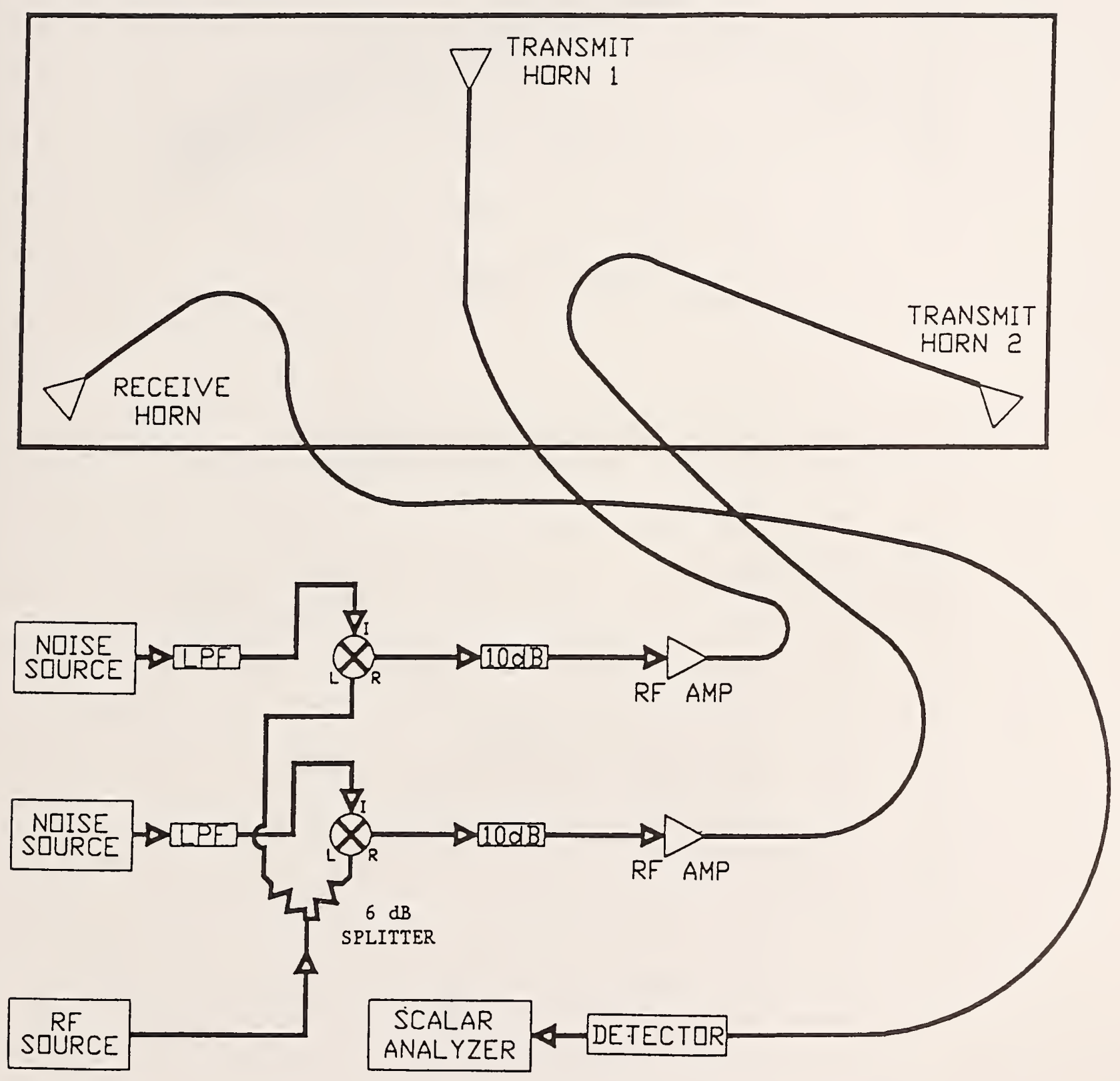

Figure 3-28. Block diagram of system for increasing test field inside reverberation chamber using two noise sources and mixers and two rf amplifiers. 

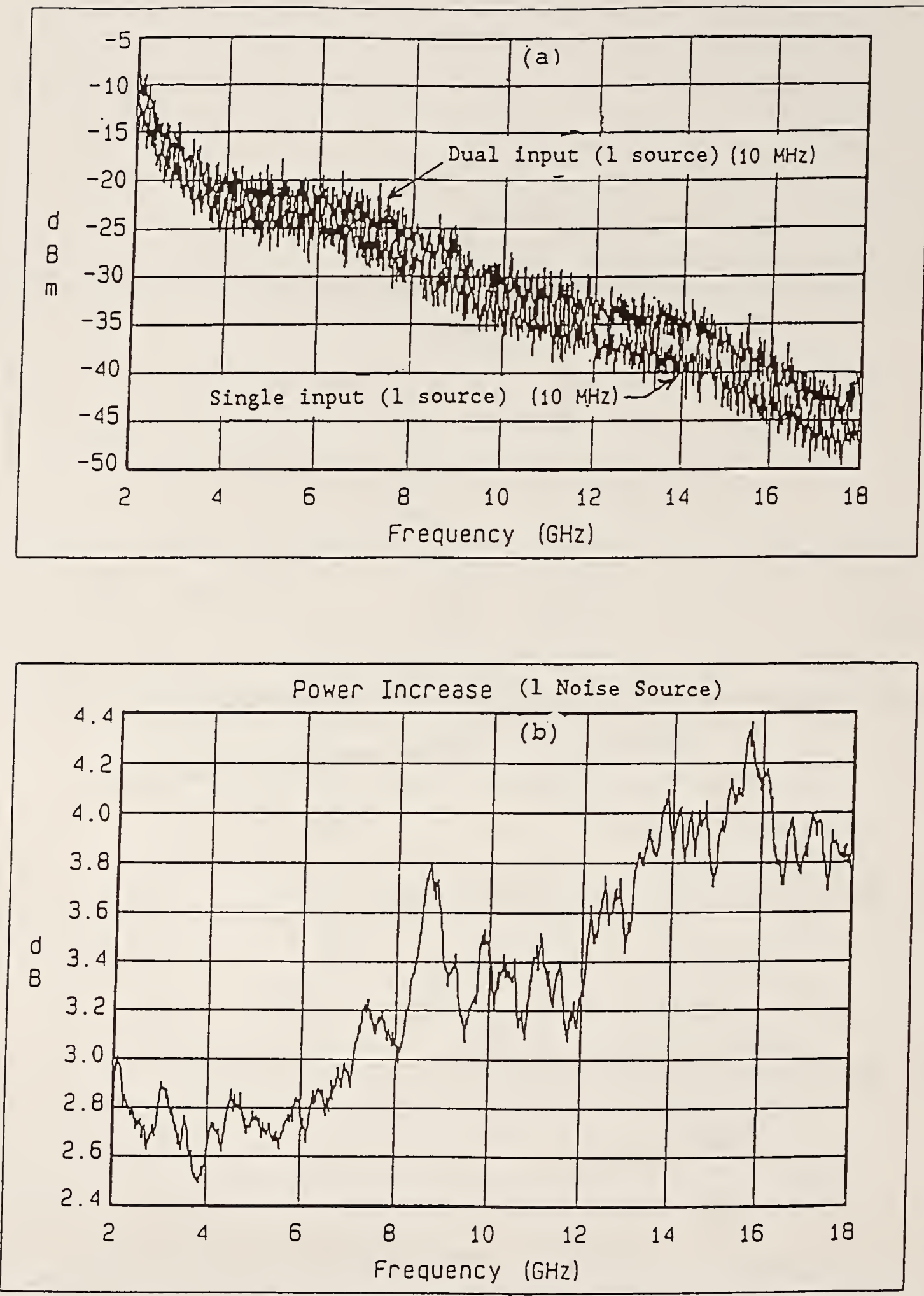

Figure 3-29. Power density measured inside NIST reverberation chamber excited using single noise source, (a) power density from single and dual inputs, (b) increase in power density from single input to dual input (Pd dual - Pd single). 

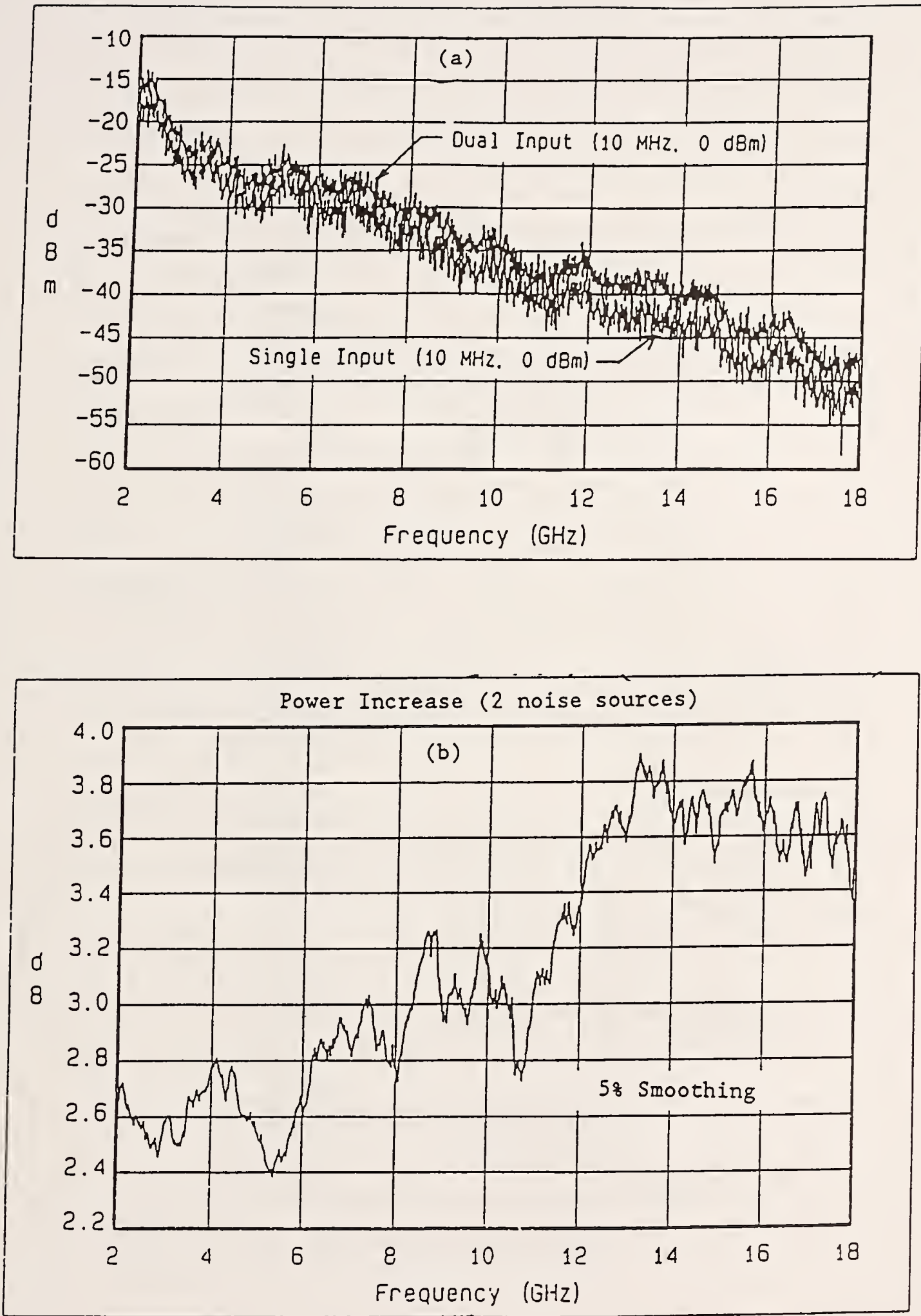

Figure 3-30. Power density measured inside NIST reverberation chamber excited using two noise sources, (a) Power density from single and dual inputs, (b) Increase in power density from single input to dual input. 


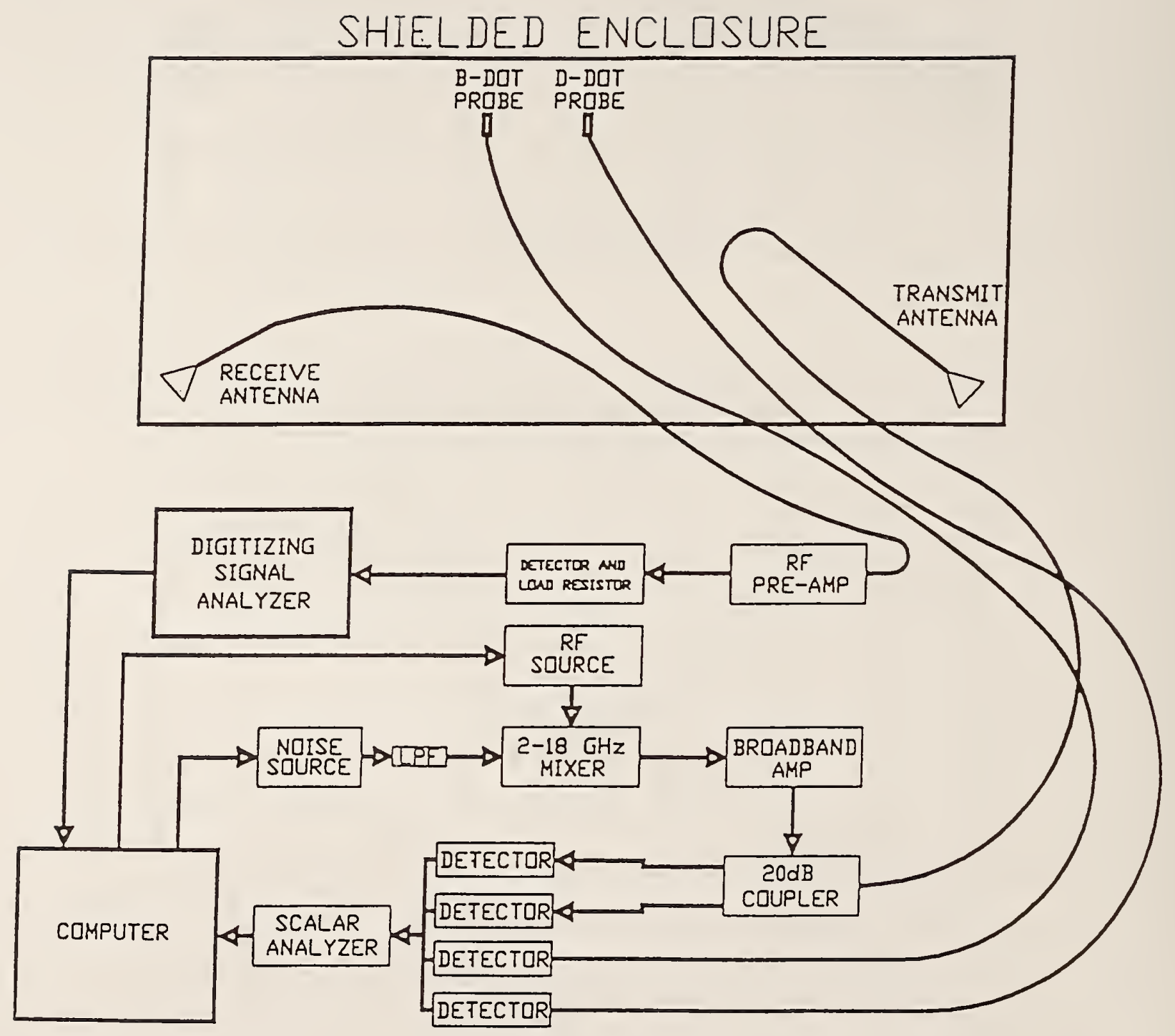

Figure 3-31. Block diagram of system for measuring transient response and field uniformity of NBWGN excited chamber. 


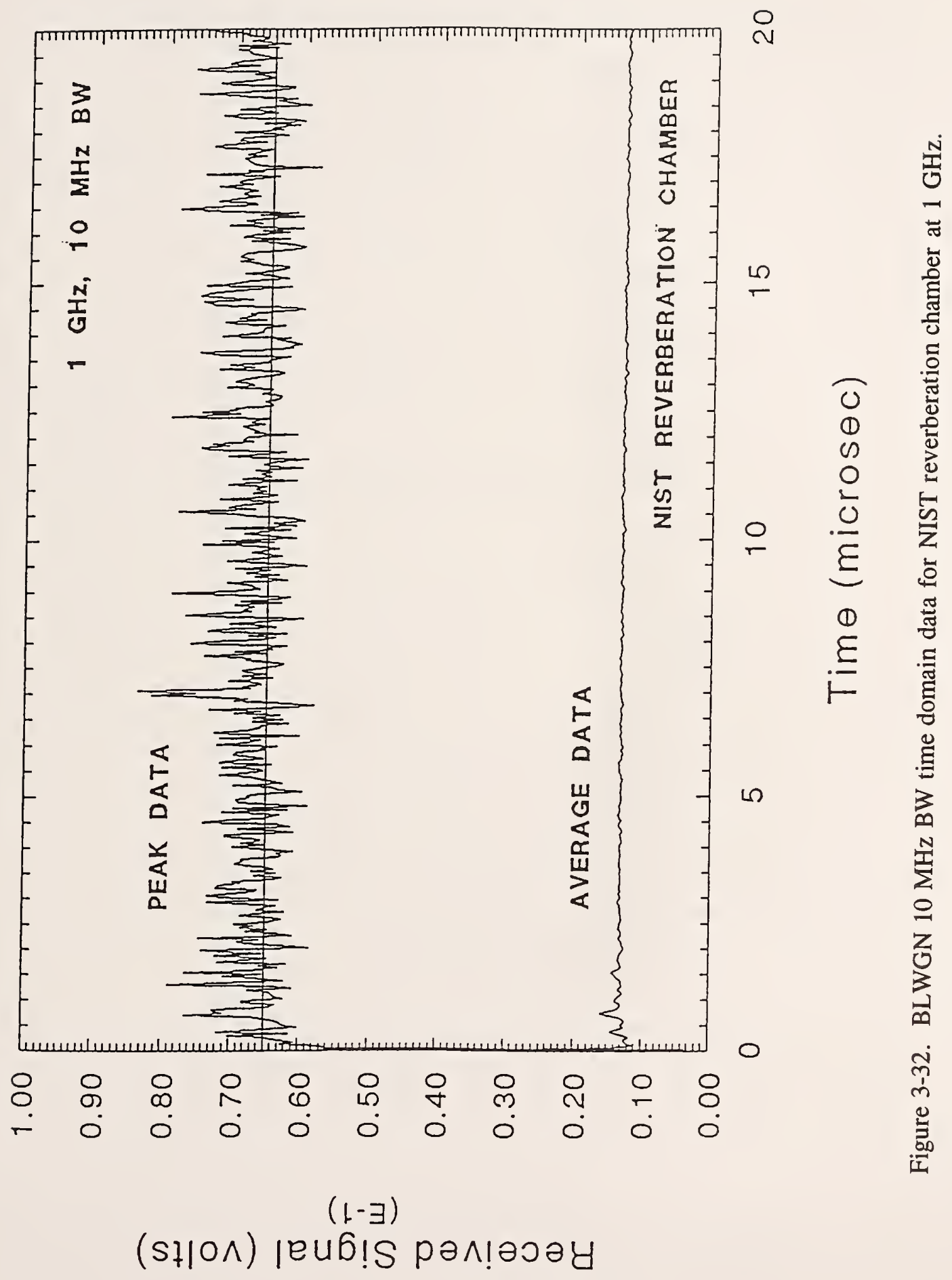




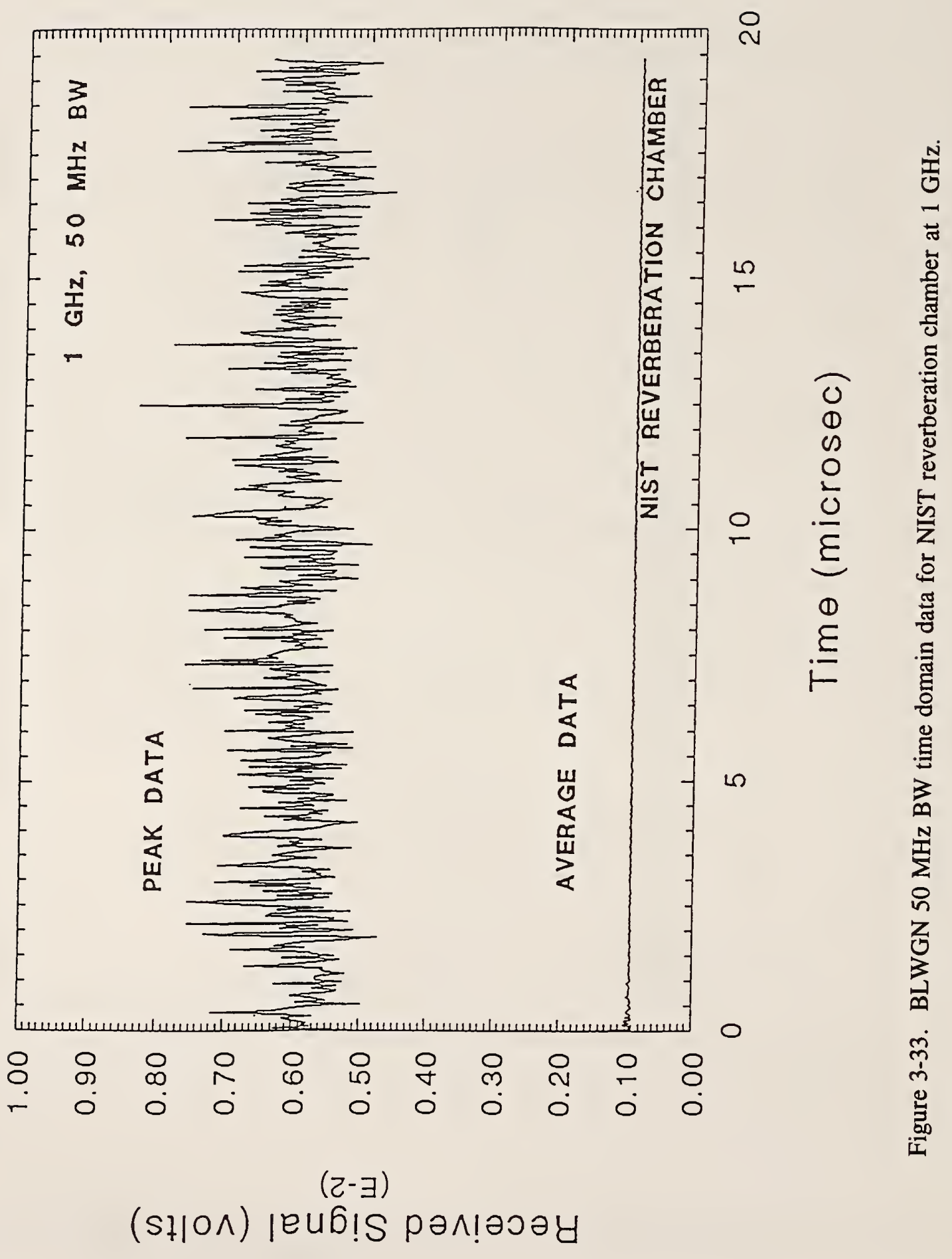




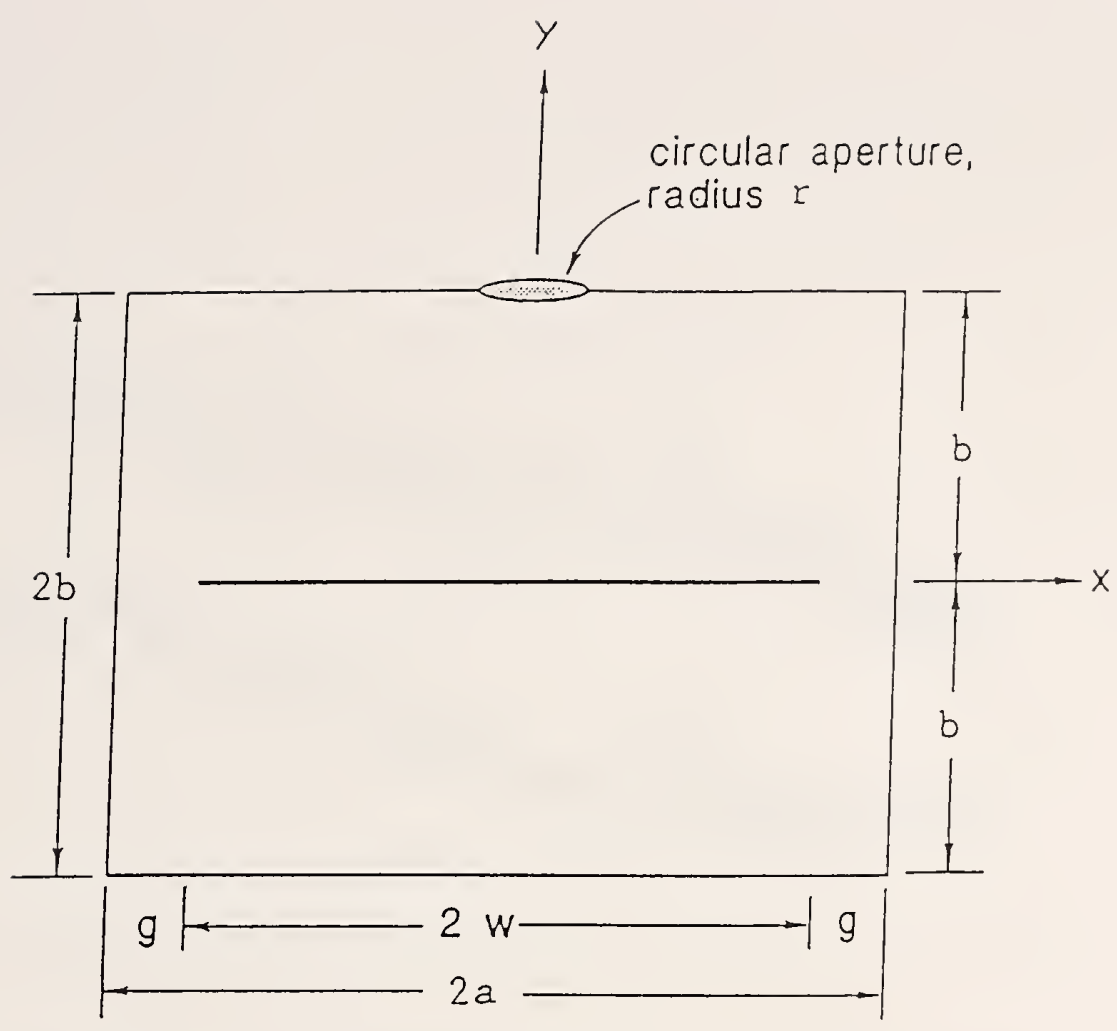

Figure 4-1. Geometry for an apertured TEM cell. 


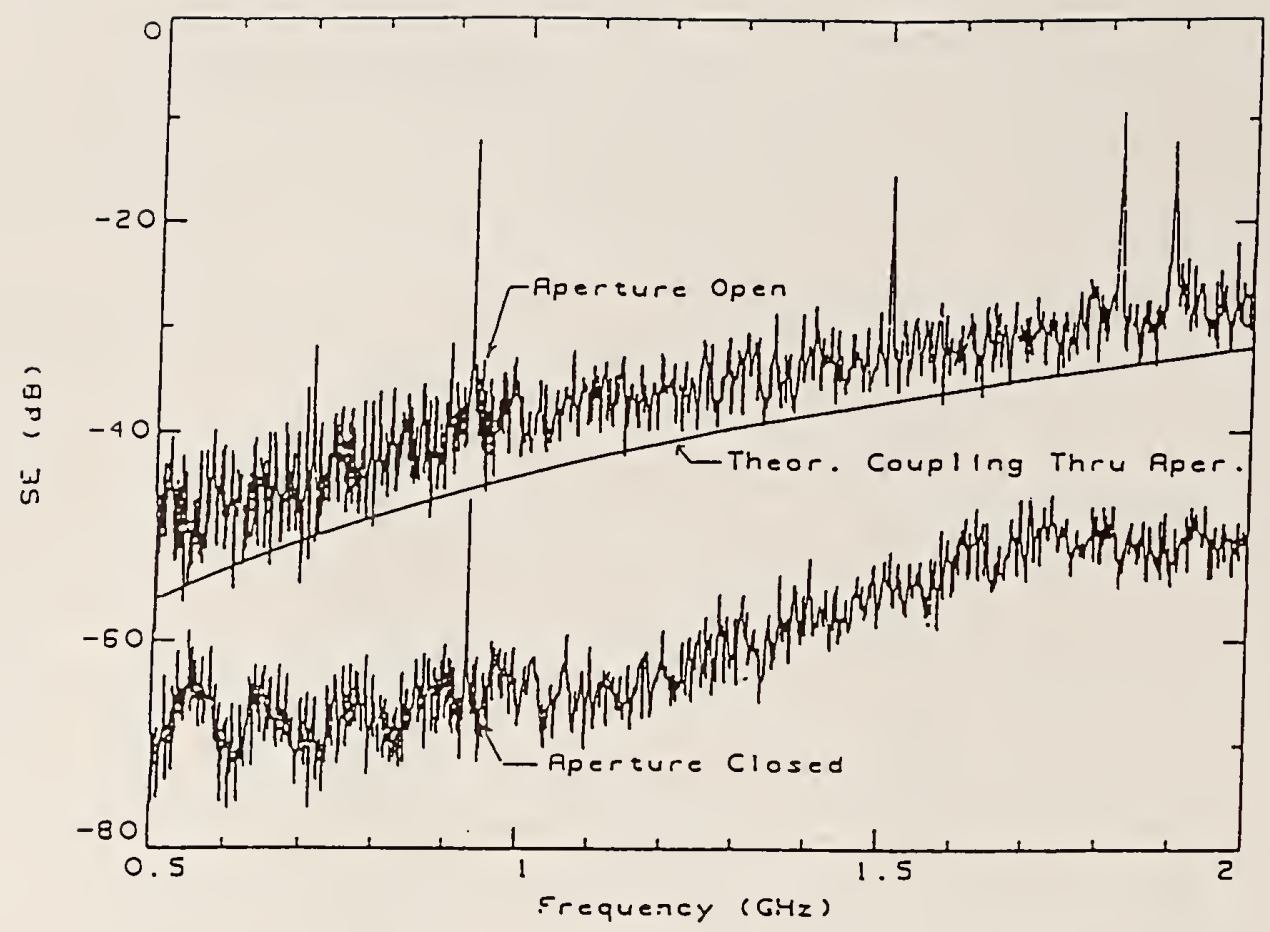

Figure 4-2. SE measurements of NIST $12 \mathrm{~cm}$ TEM cell obtained using NIST reverberating chamber with mechanical mode-mixing.

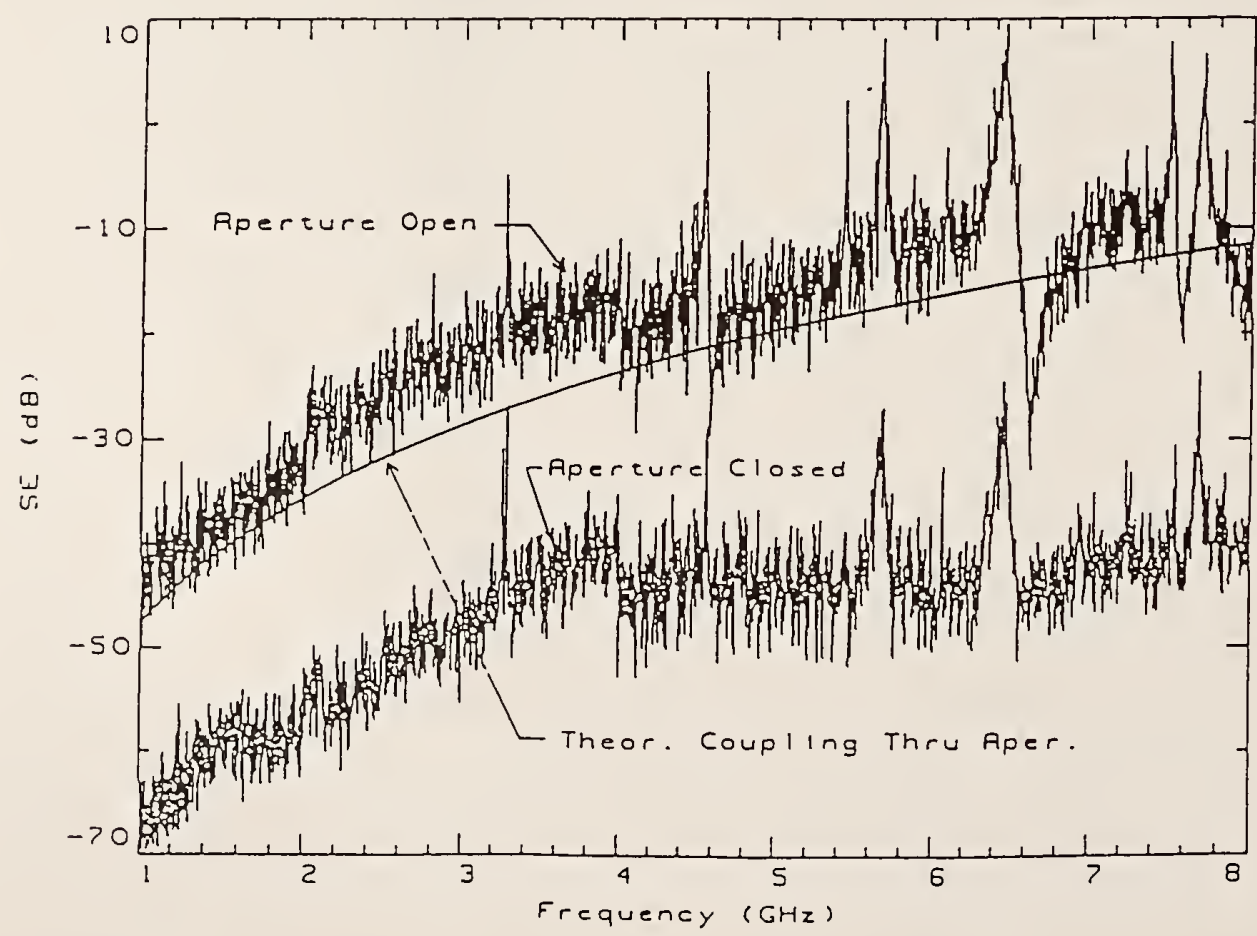

Figure 4-3. SE measurements of NIST $6 \mathrm{~cm}$ TEM cell obtained using NIST reverberating chamber with mechanical mode-mixing. 


\section{GATING FUNCTION}

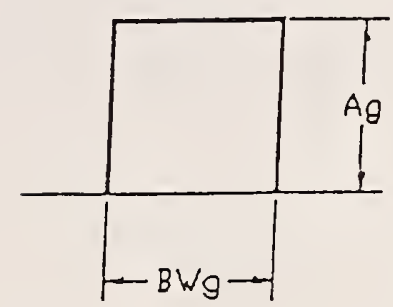

CROSS SECTION

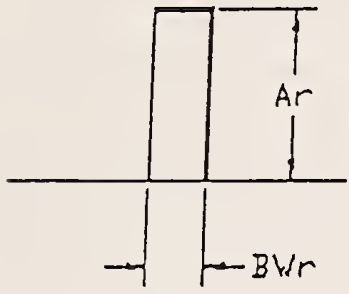

CONVOLUTION/(AgBN')

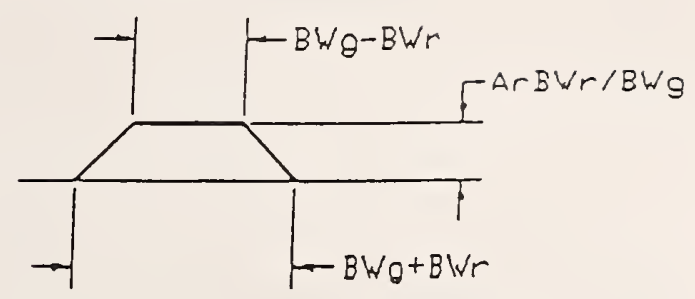

Figure 4-4. Effect of cross-section bandwidth smaller than bandwidth of gating function.

GATING FUNCTION

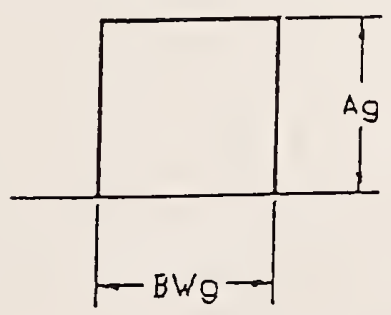

CROSS SECTION
CONVOLUTION/(AgBTg)

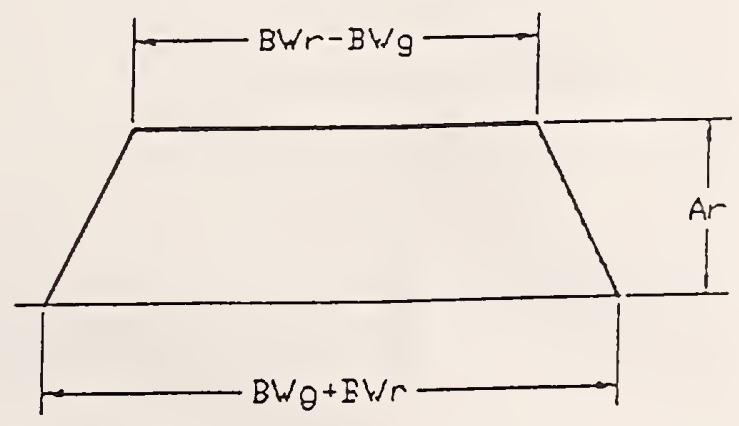

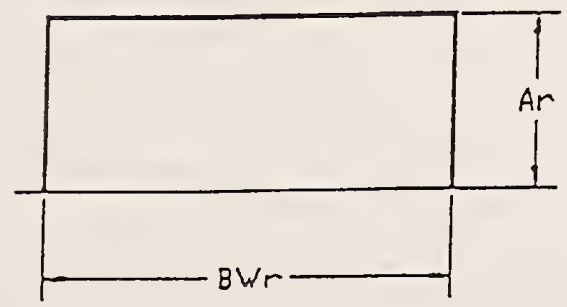

Figure 4-5. Effect of cross-section bandwidth greater than bandwidth of gating function. 


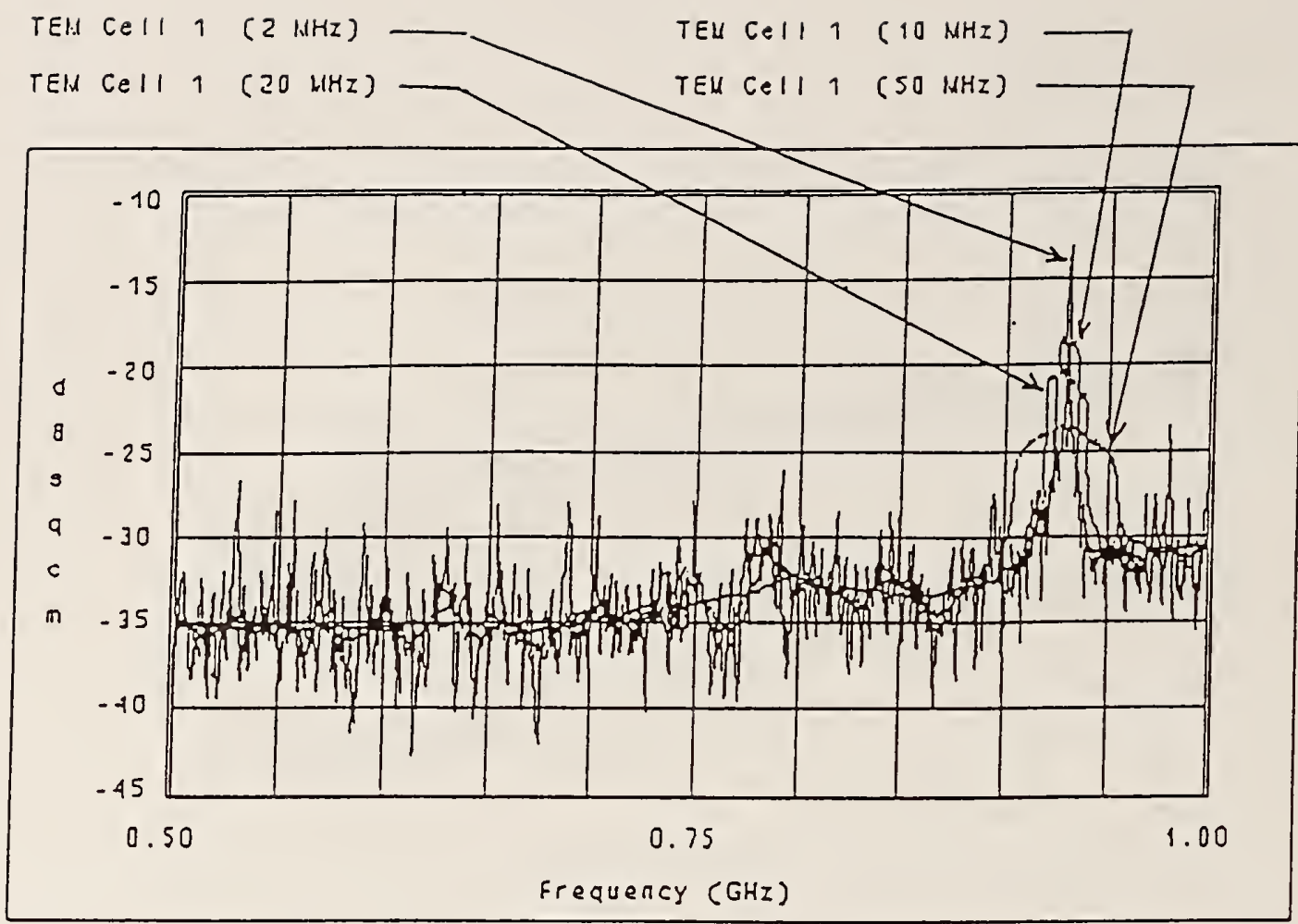

Figure 4-6. Narrow cross-section resonance of $12 \mathrm{~cm}$ TEM cell measured using BLWGN.
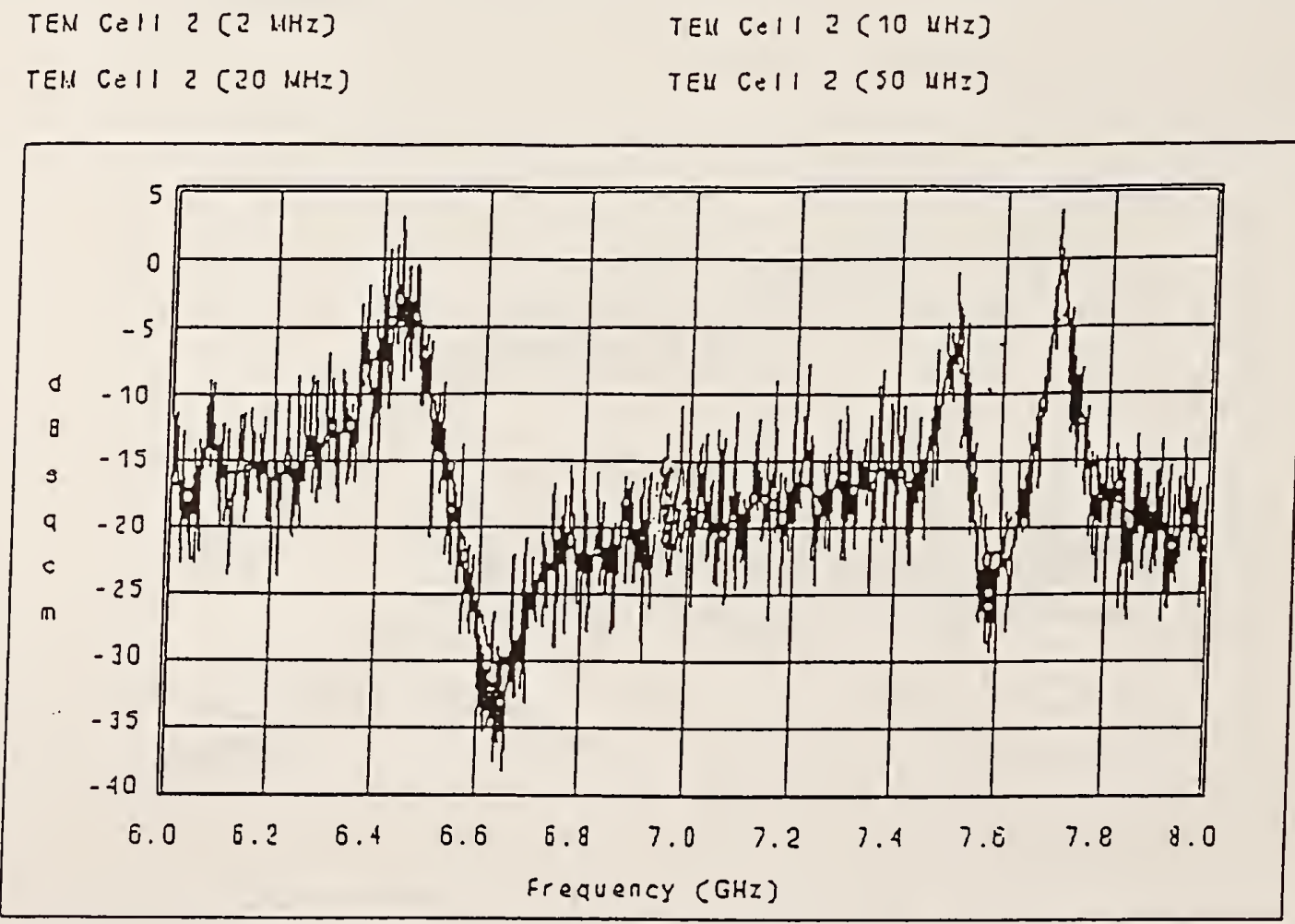

Figure 4-7. Wide cross-section resonance of $6 \mathrm{~cm}$ TEM cell measured with BLWGN. 


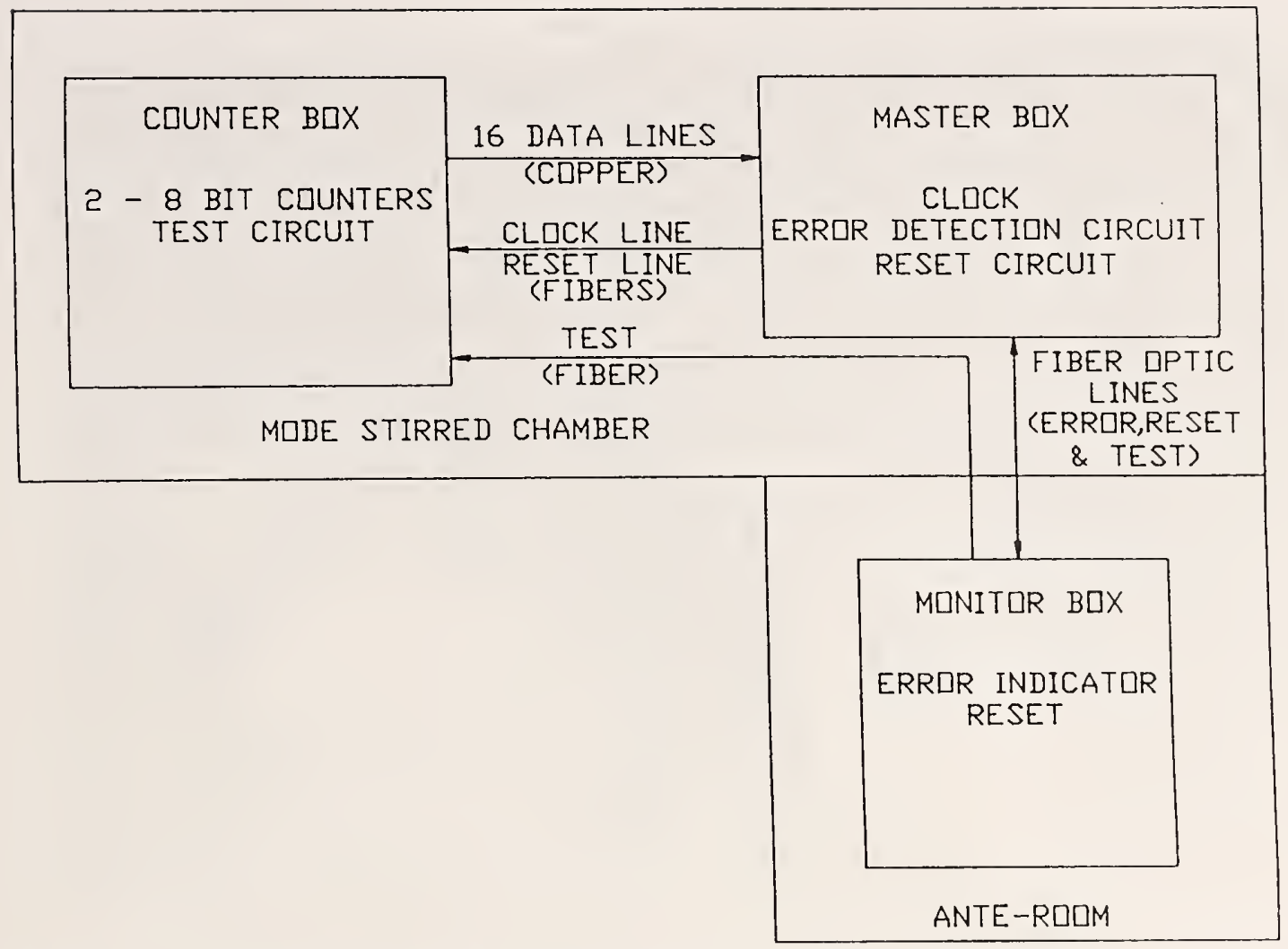

Figure 4-8. Common test article showing interconnections and functions.

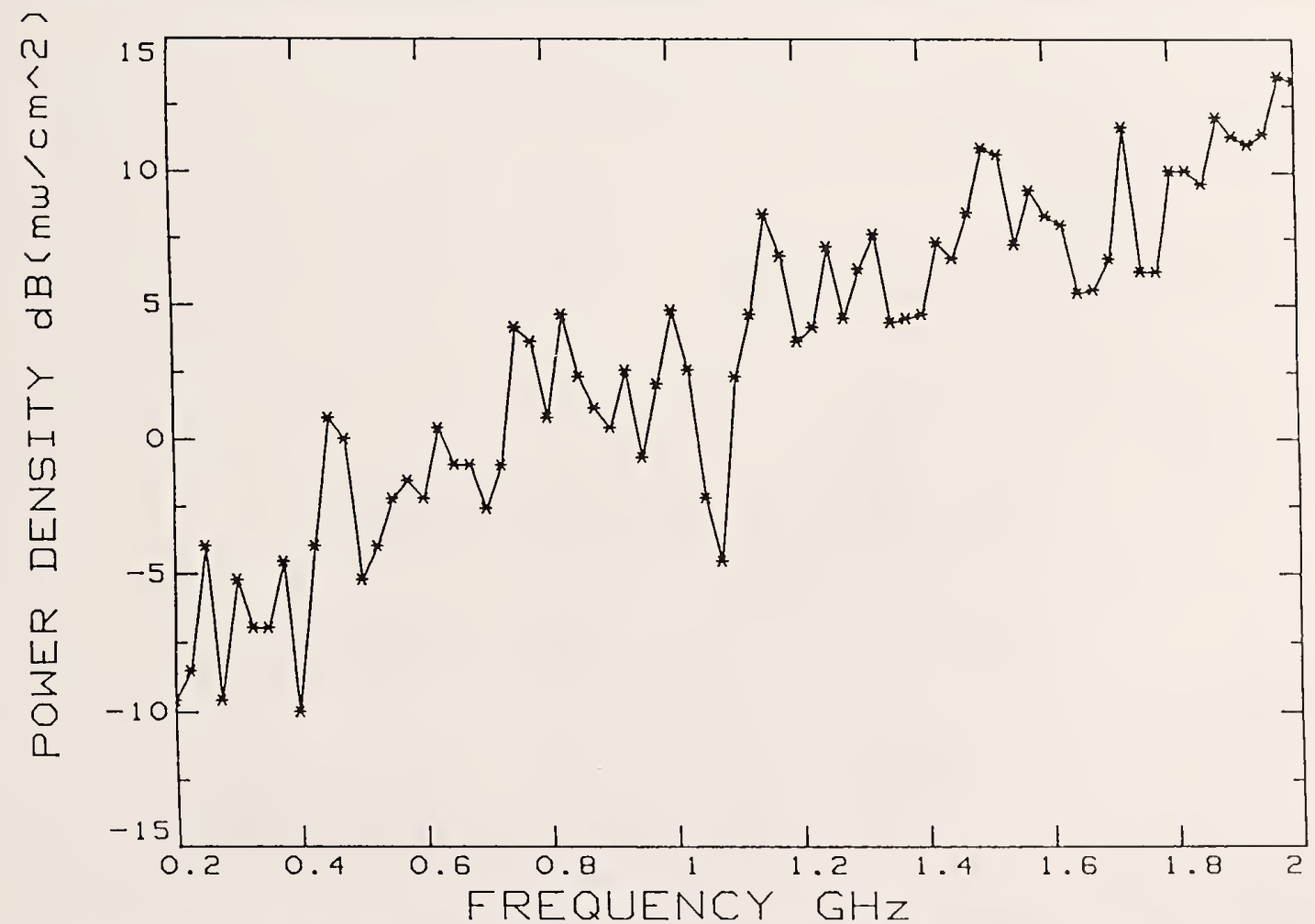

Figure 4-9. Susceptibility profile of common test article obtained using CW excitation in the NSWCDD mechanically mode-mixed reverberation chamber. 


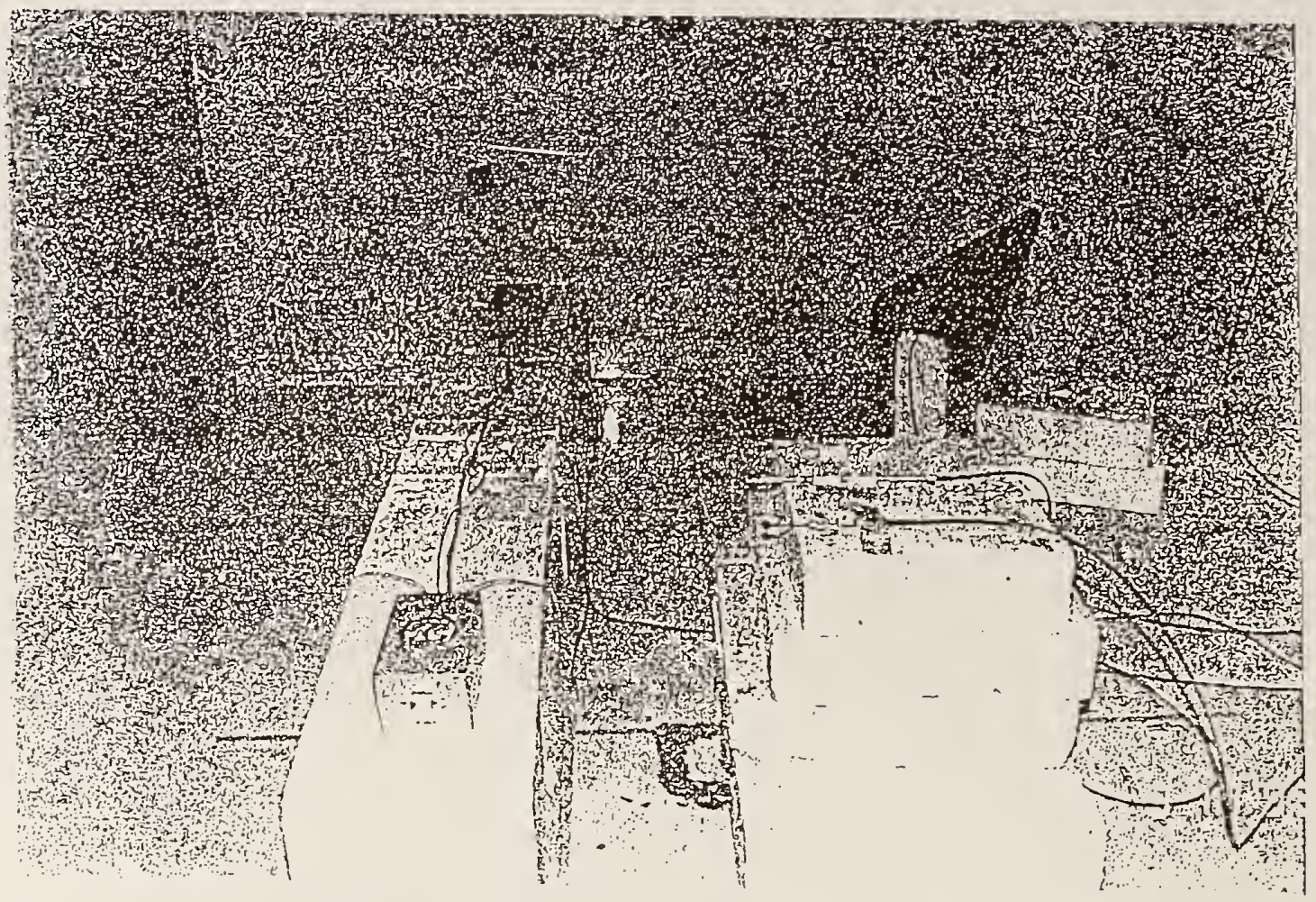

Figure 4-10. Set-up for performing susceptibility tests of common test article in a reverberation chamber.

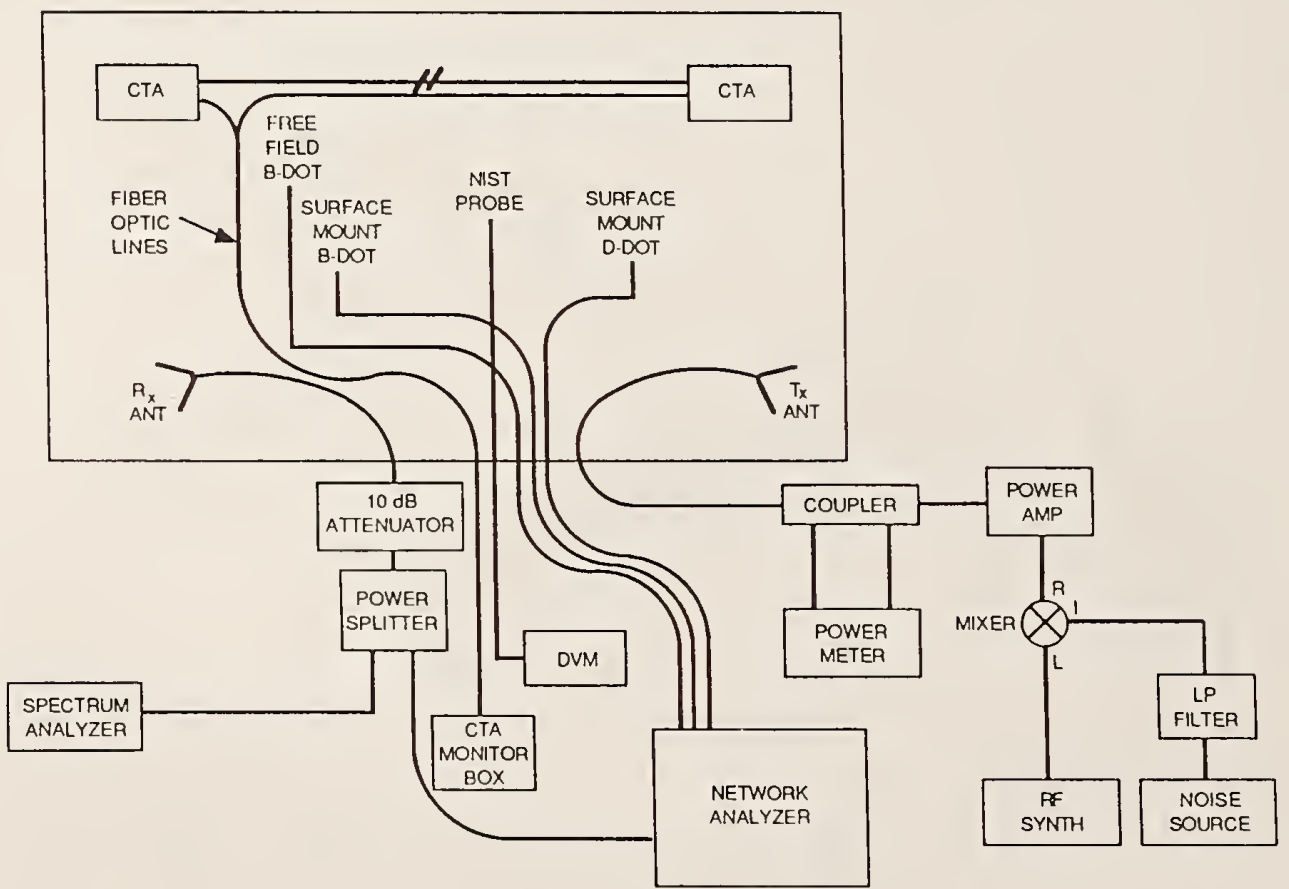

Figure 4-11. Block diagram of system for performing susceptibility tests of common test article in NIST reverberation chamber using BLWGN excitation. 


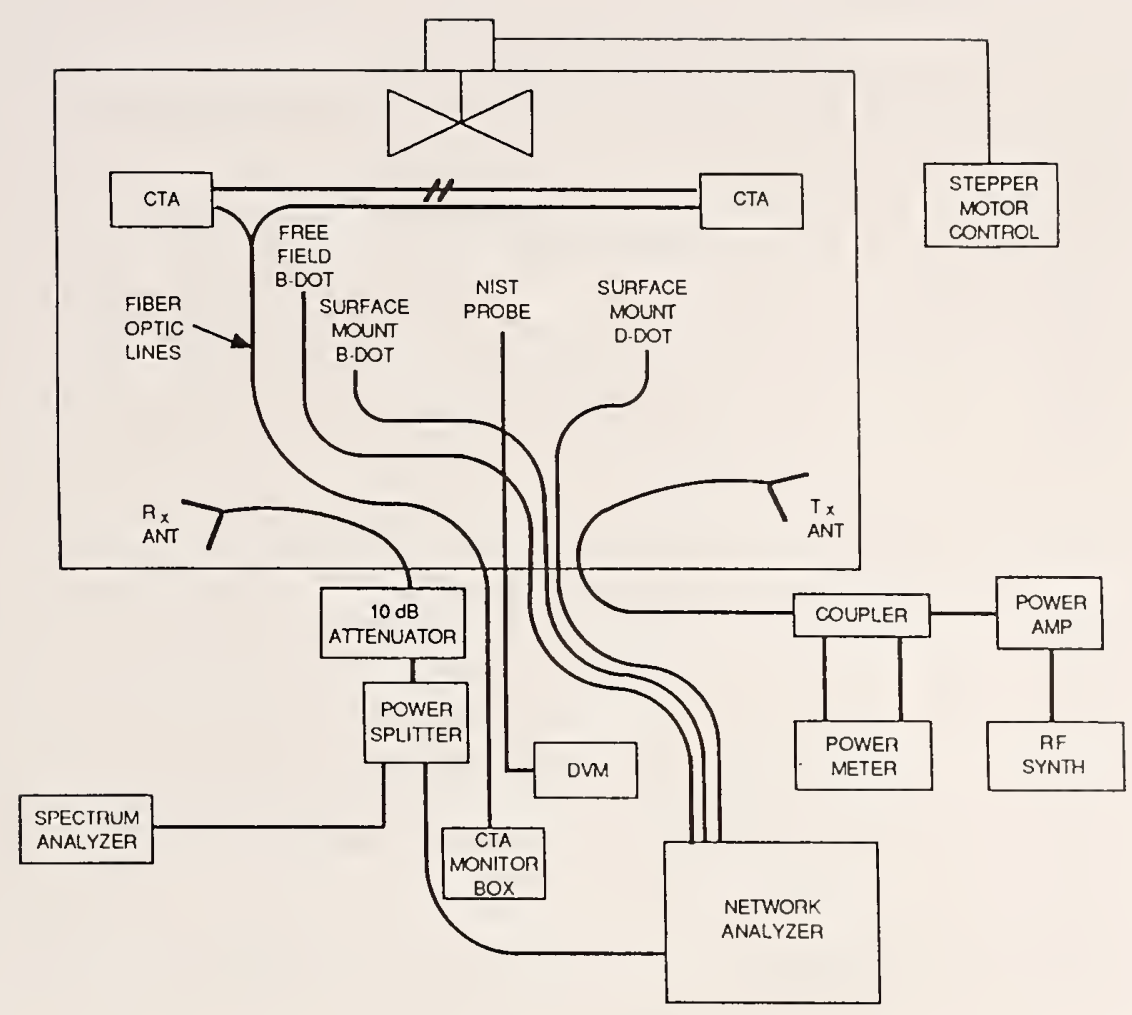

Figure 4-12. Block diagram of system for performing susceptibility tests of common test article in NIST reverberation chamber using $\mathrm{CW}$ excitation with mechanical mode-mixing. 


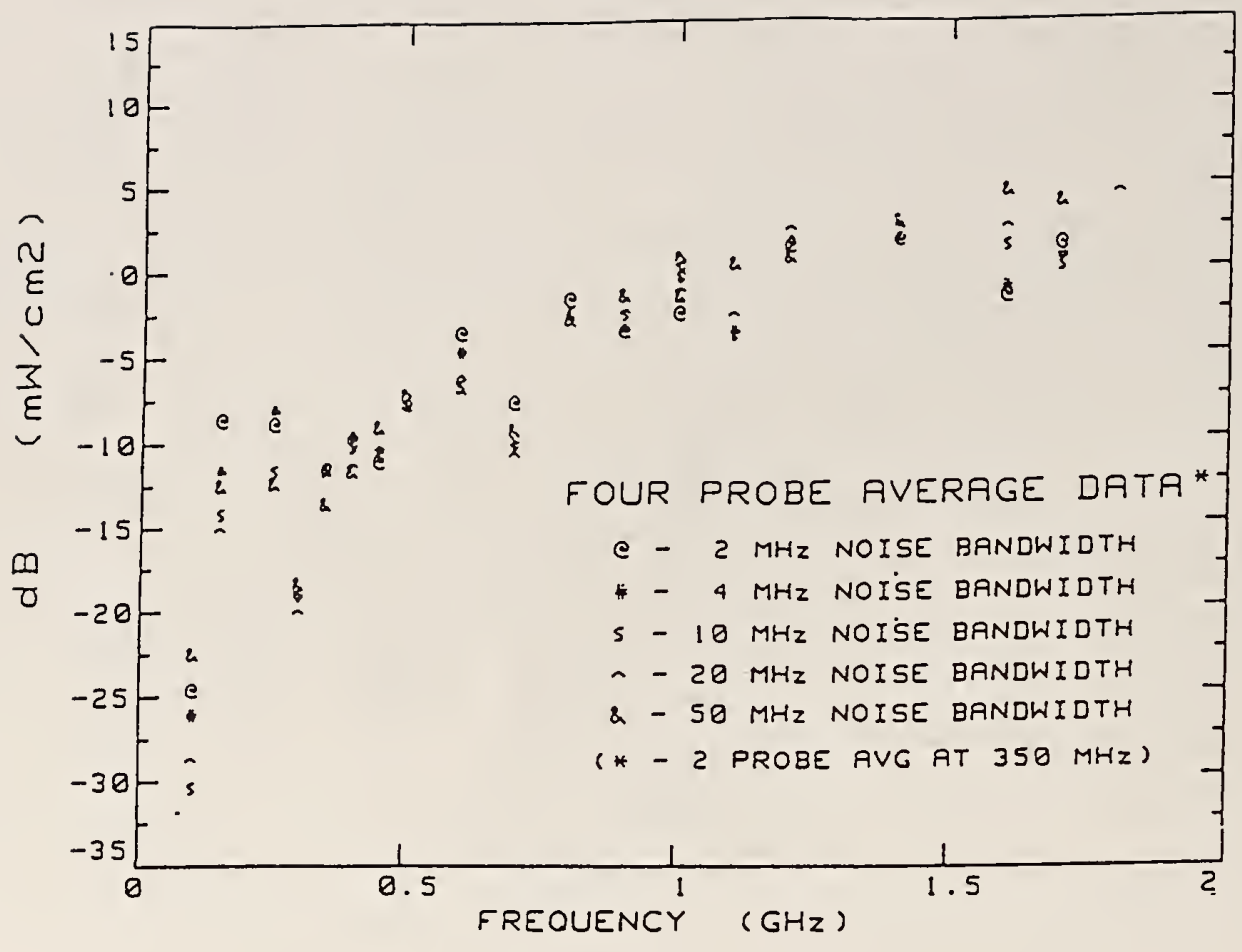

Figure 4-13. Susceptibility profile of common test article obtained with BLWGN (5 bandwidths).

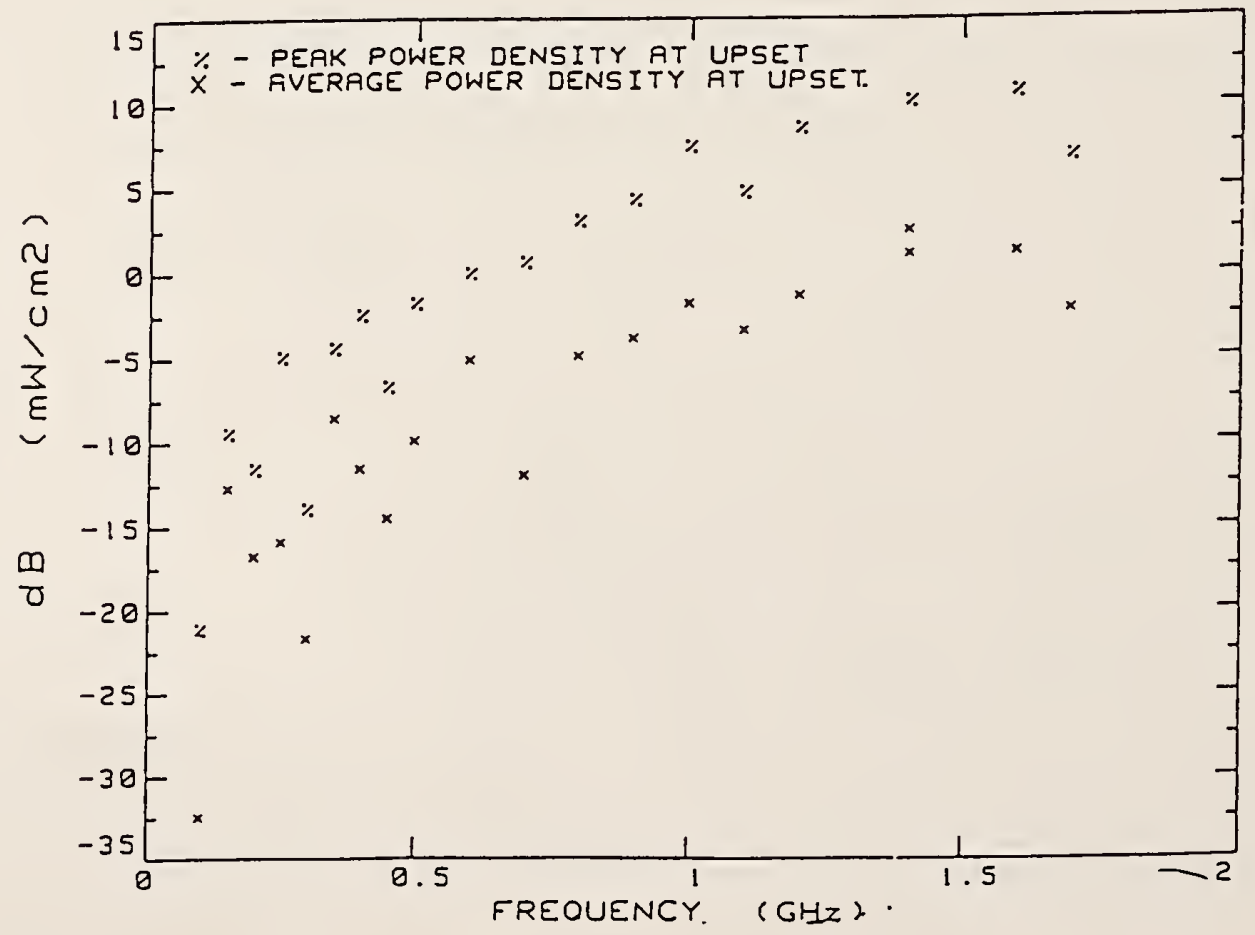

Figure 4-14. Peak and average power susceptibility levels of common test article obtained with CW excitation and mechanical mode mixing. 


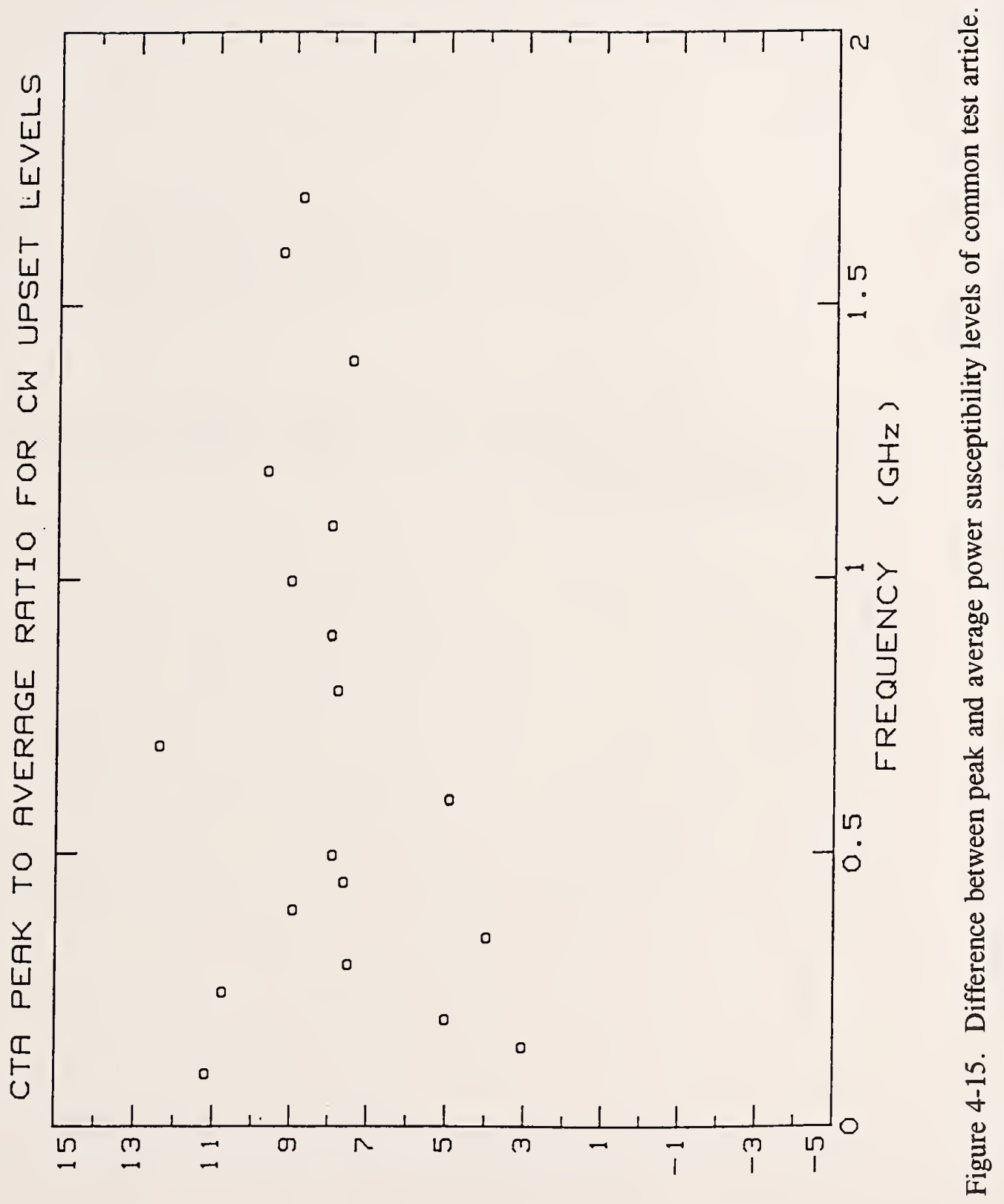

$(\exists \supset \forall \forall \exists \wedge \forall \quad O \perp \quad \forall \forall \exists d \forall \perp\urcorner \exists \square) \quad$ \&p 


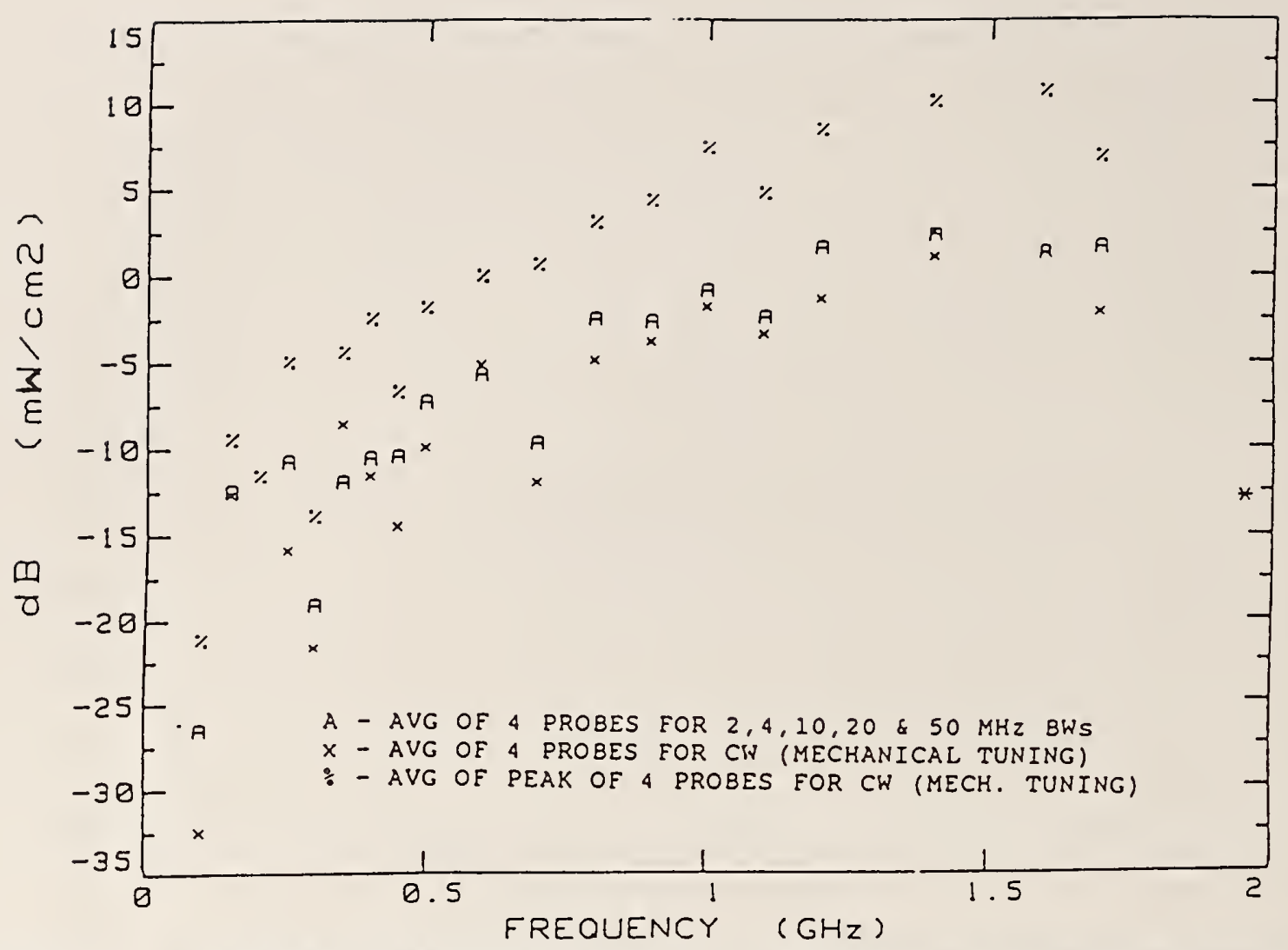

Figure 4-16. Comparison of BLWGN and average CW (mechanical mode mixing) power densities data for common test article upset.

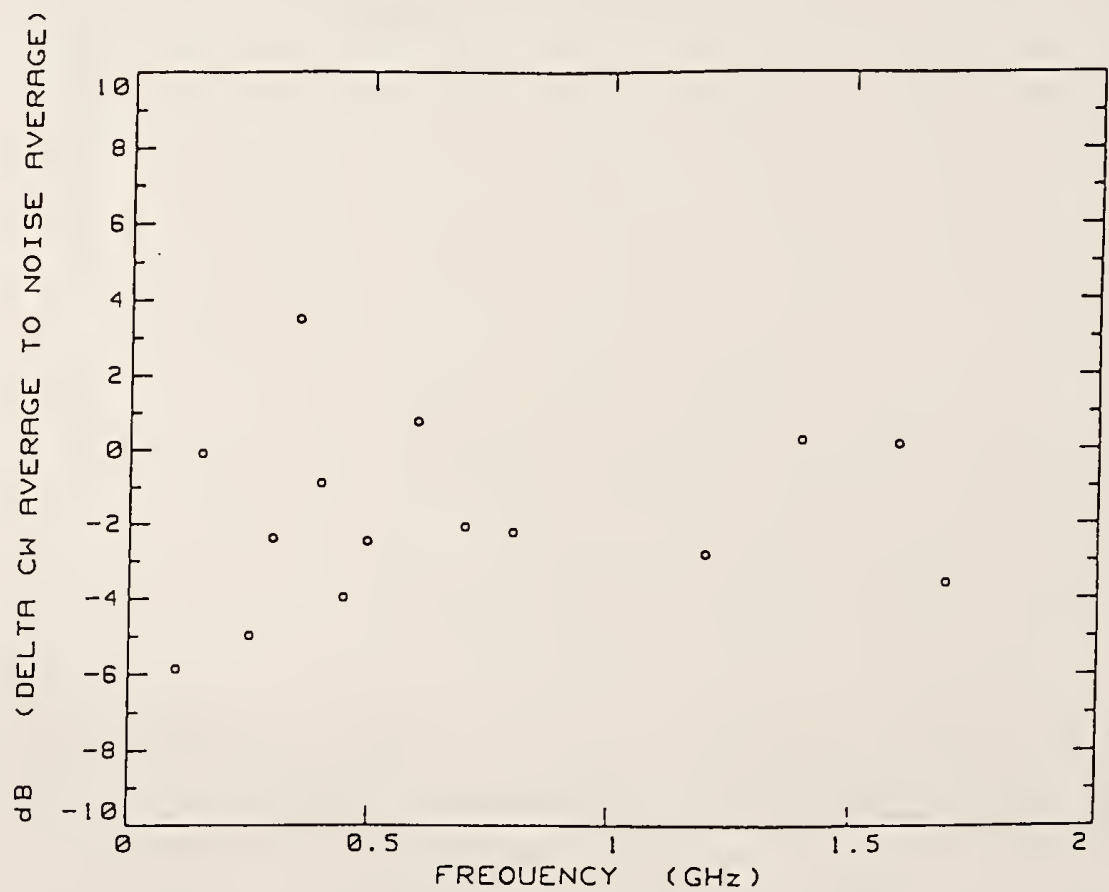

Figure 4-17. Difference between CW (mechanical mode mixing) average and BLWGN data for common test article upset. 


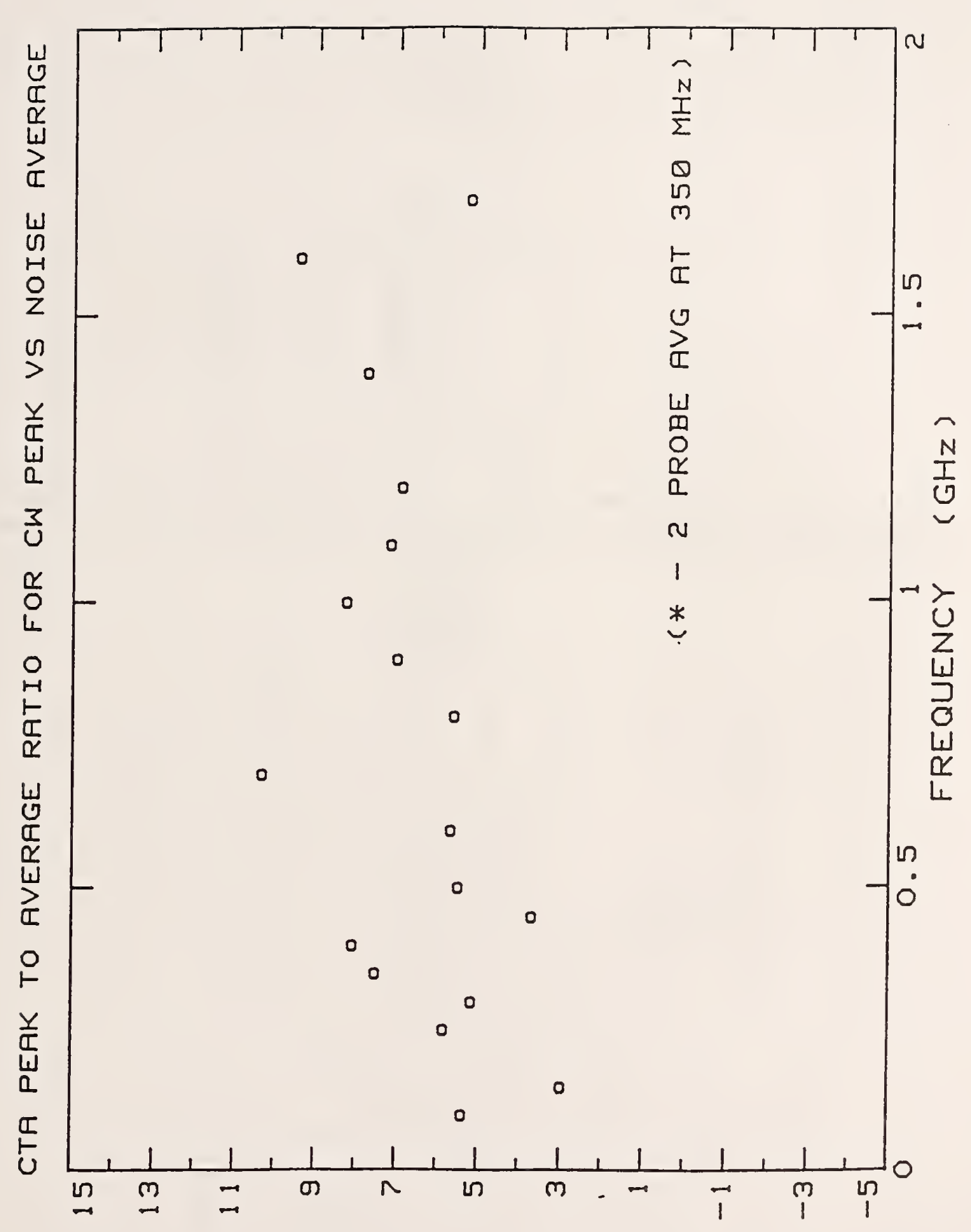

$(5 \wedge \forall \exists S I O N$ - $\forall \forall \exists d M \supset \forall \perp 7 \exists \square)$ gP

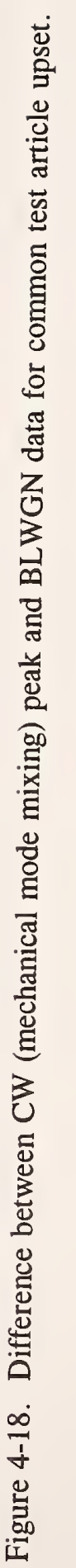




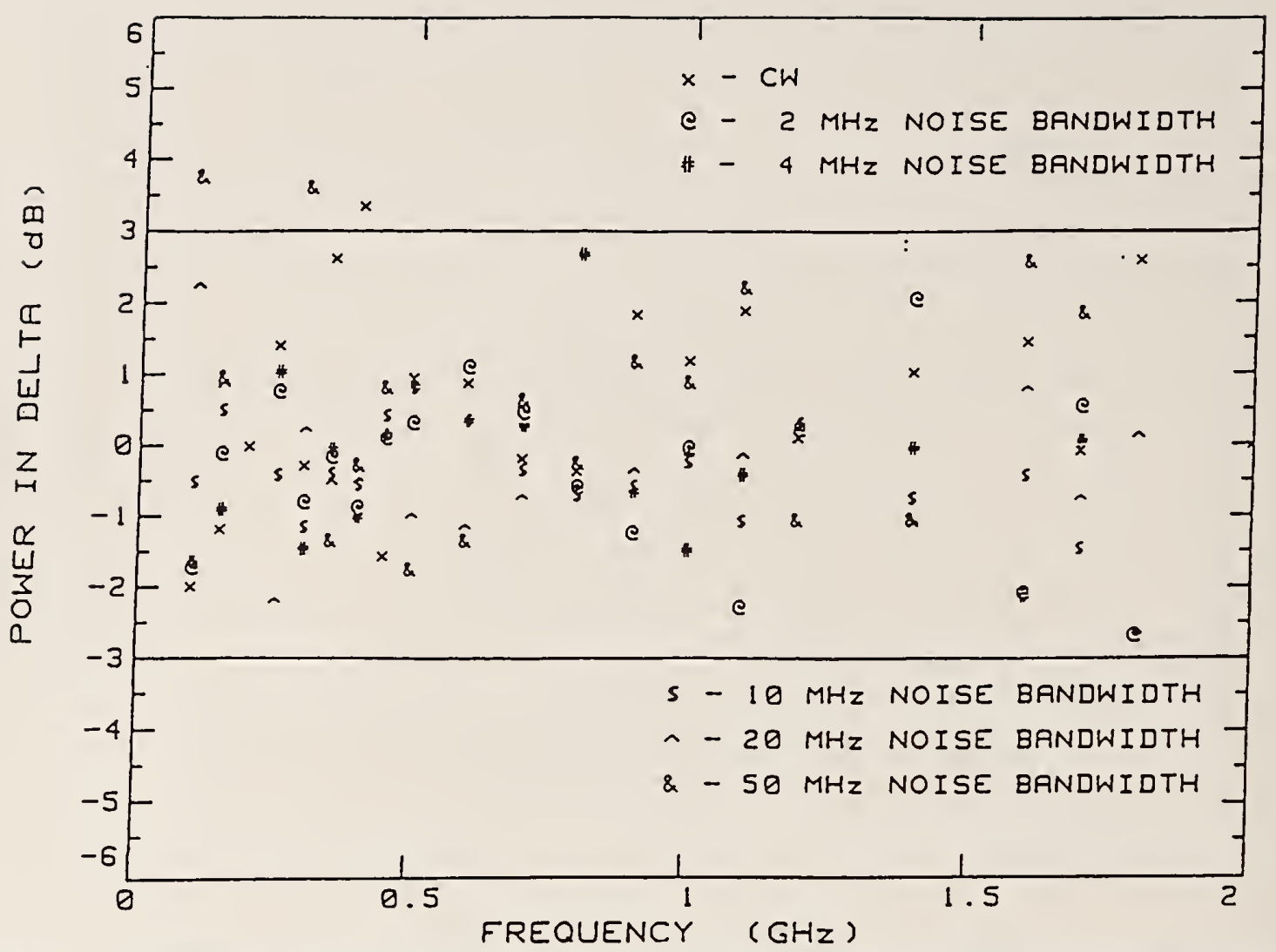

Figure 4-19. Difference between input power to NIST reverberation chamber required for upset of common test article determined from average of all probe and antenna measurements and from each individual sensor. 


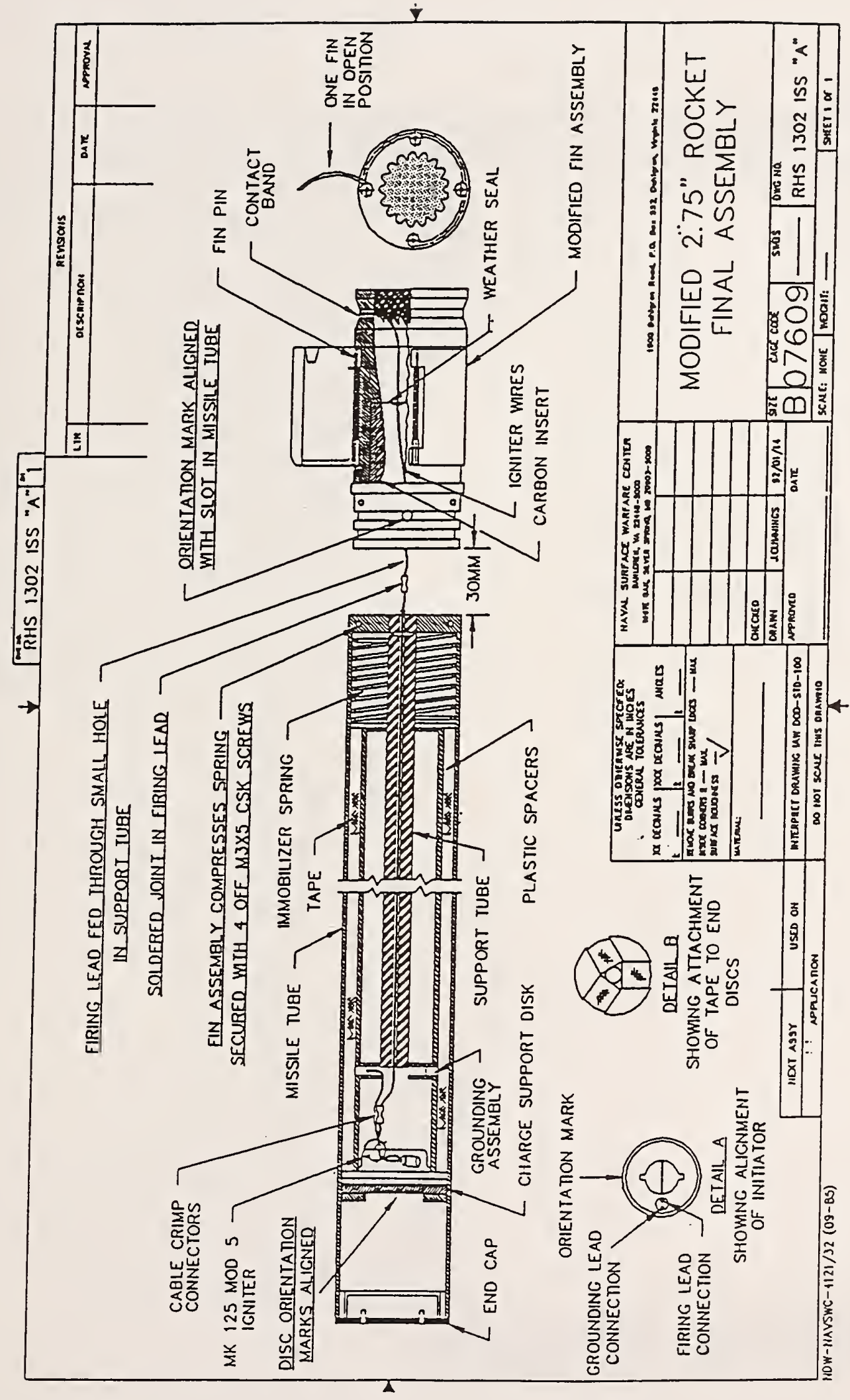

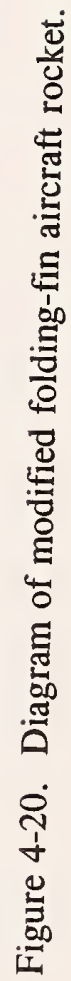




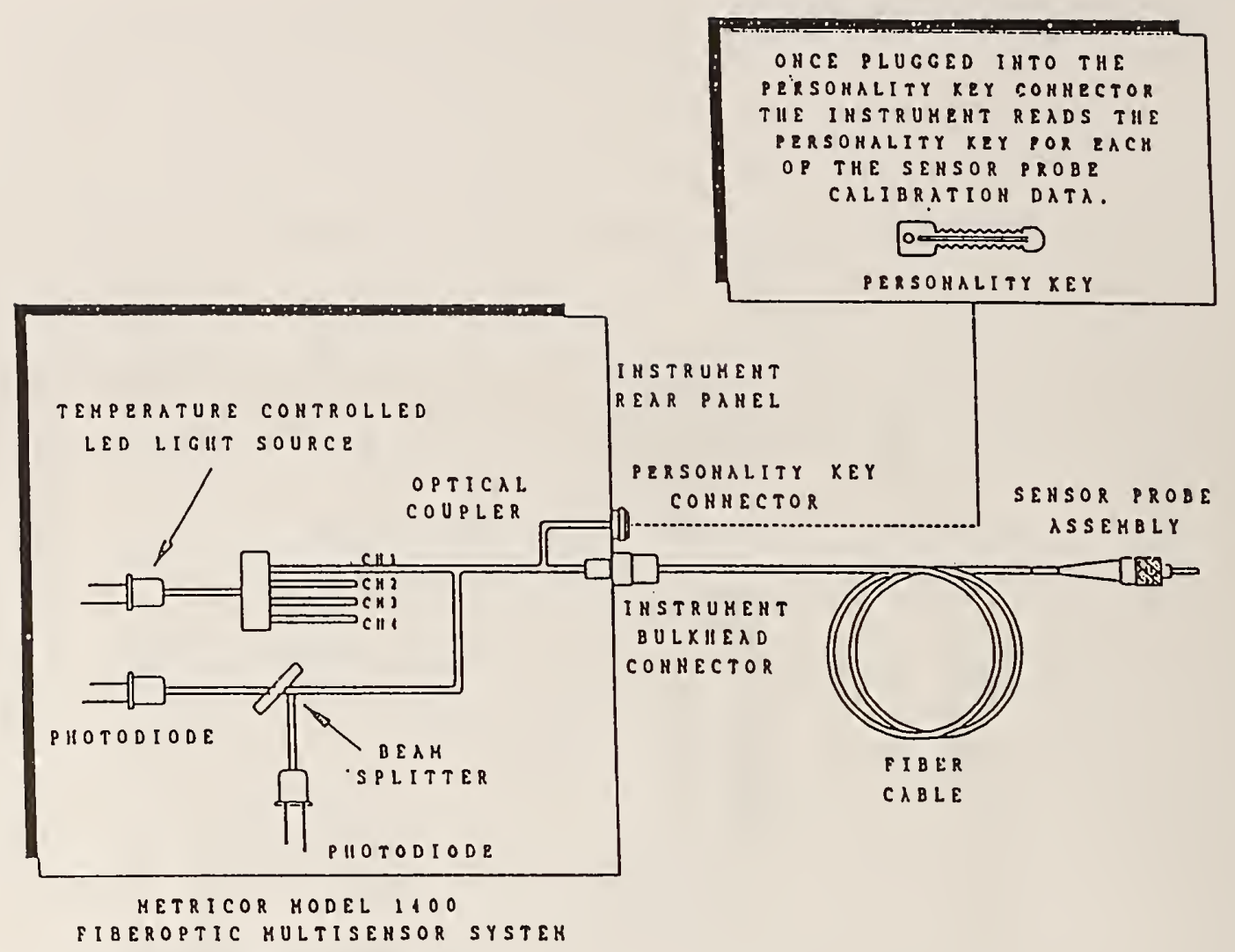

Figure 4-21. Diagram of MetriCor system.

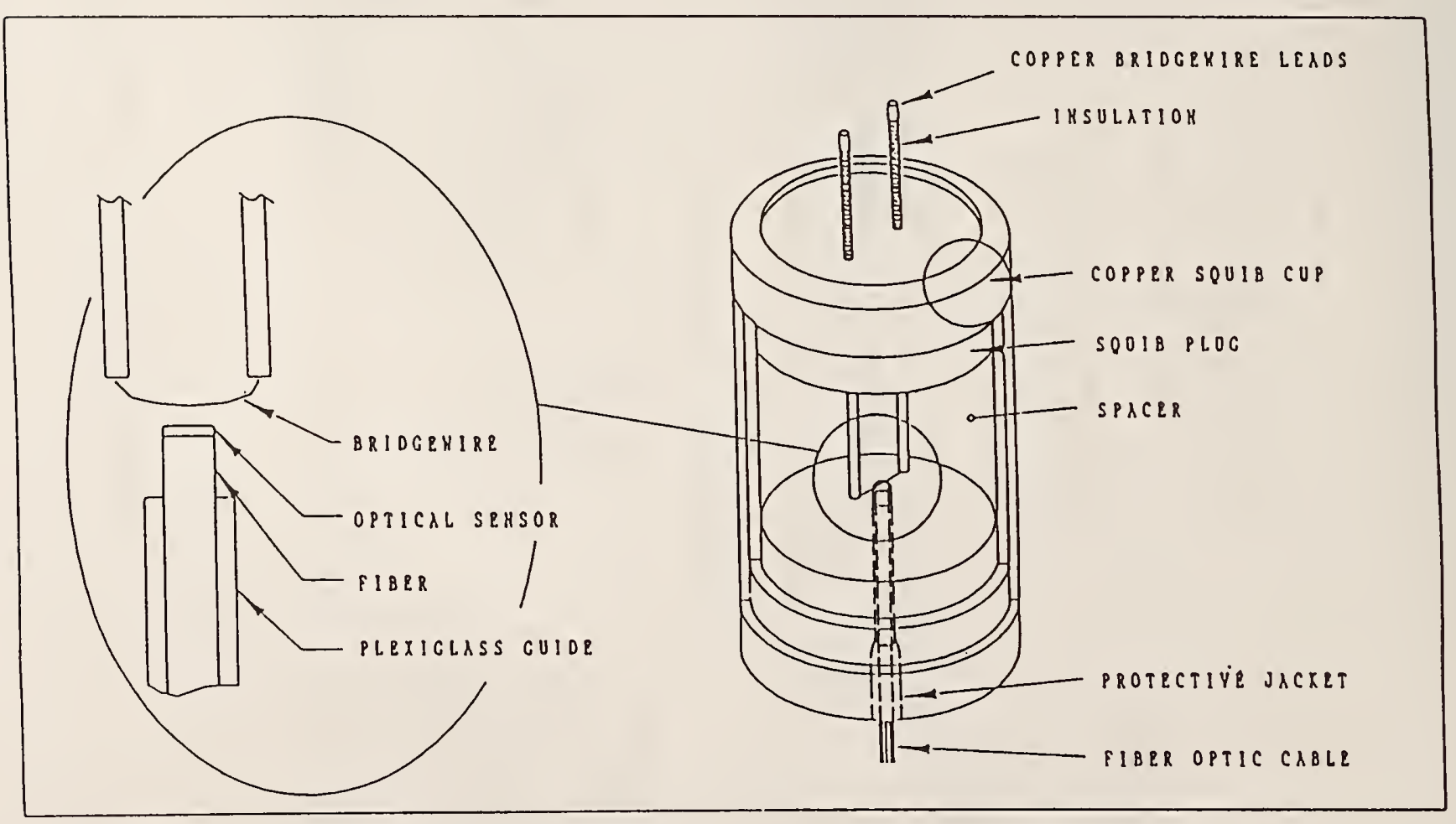

Figure 4-22. Diagram of instrumented EED. 


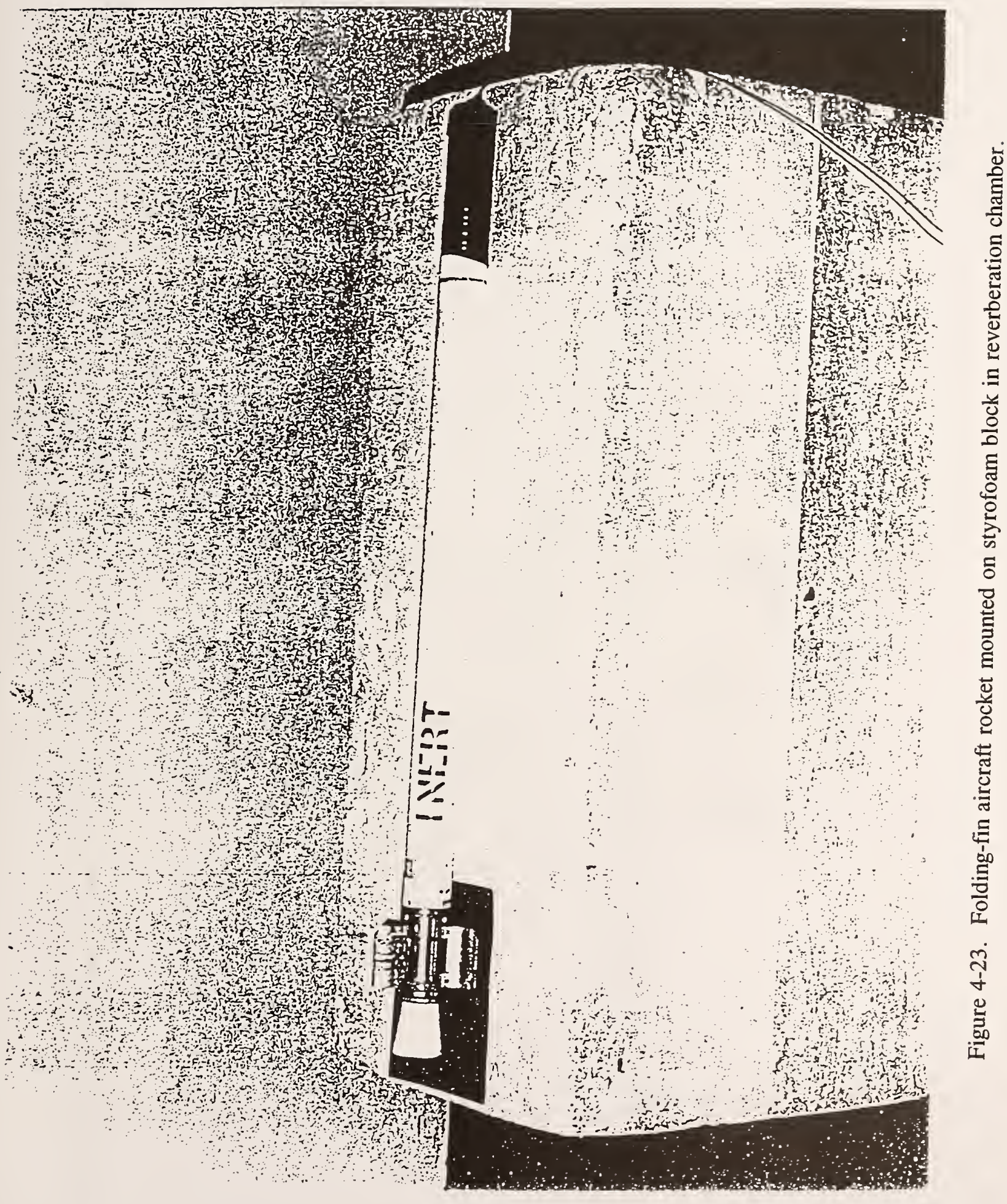




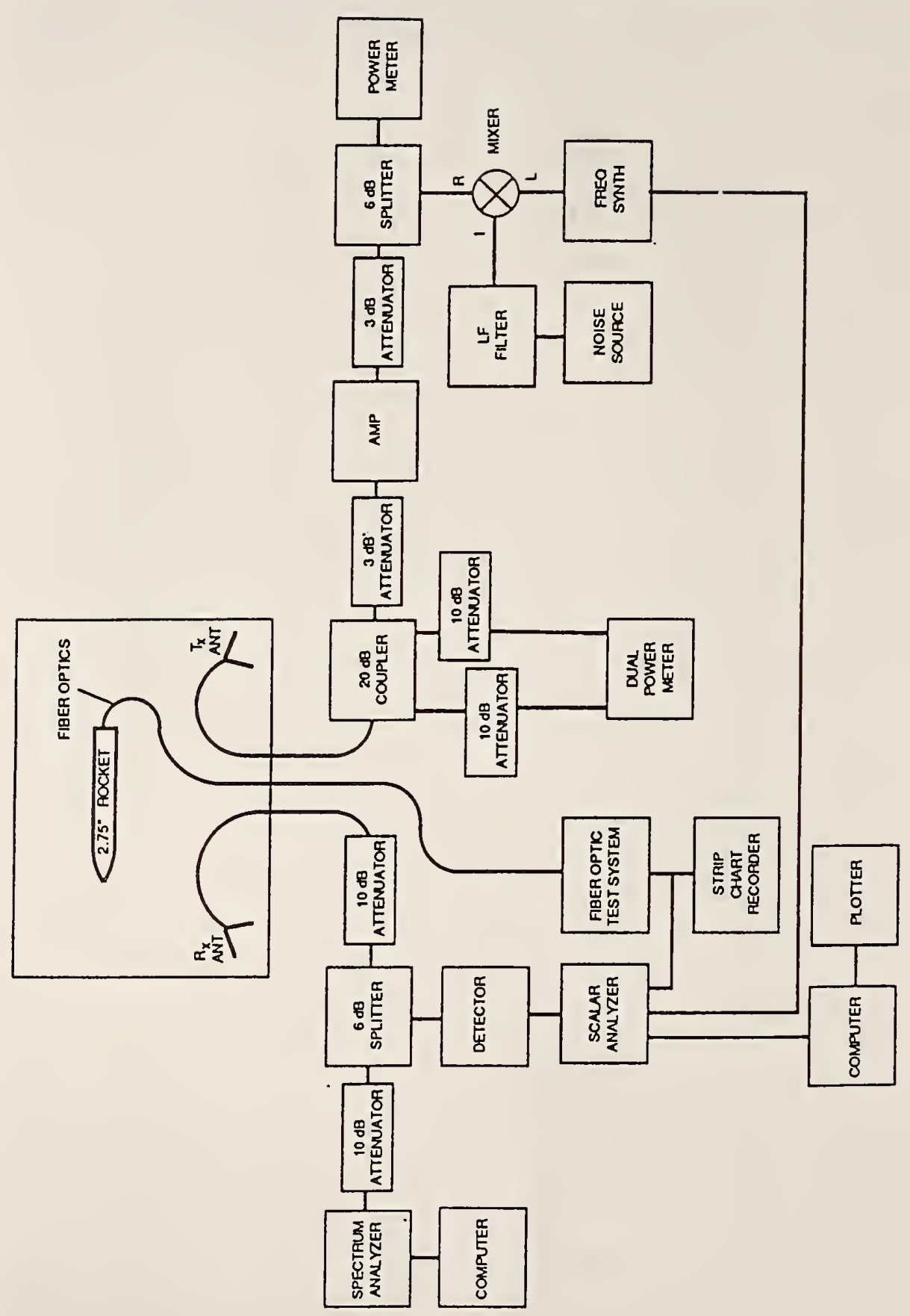

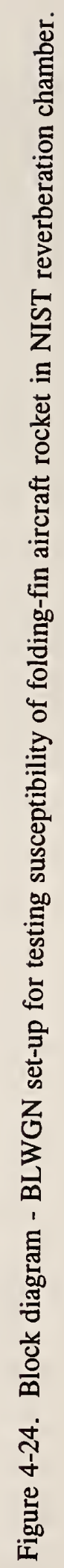




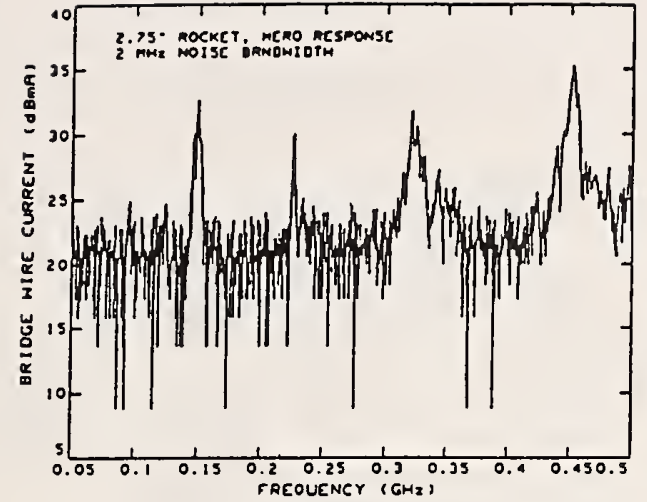

(a)

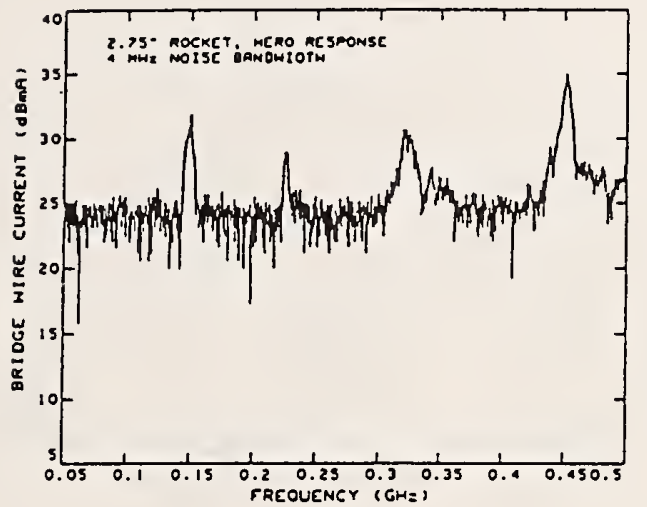

(b)

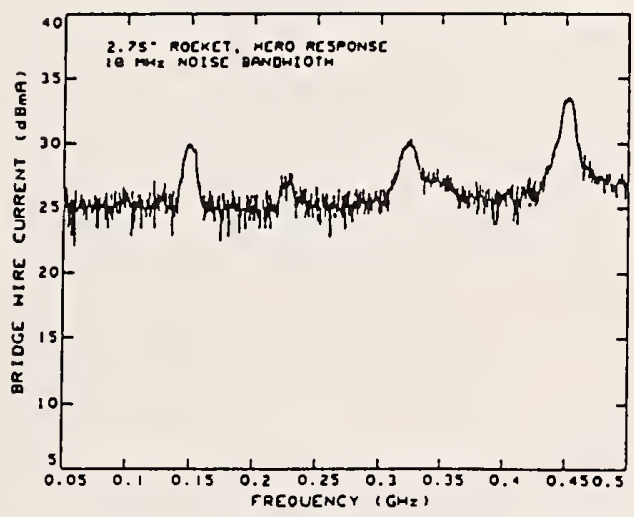

(c)

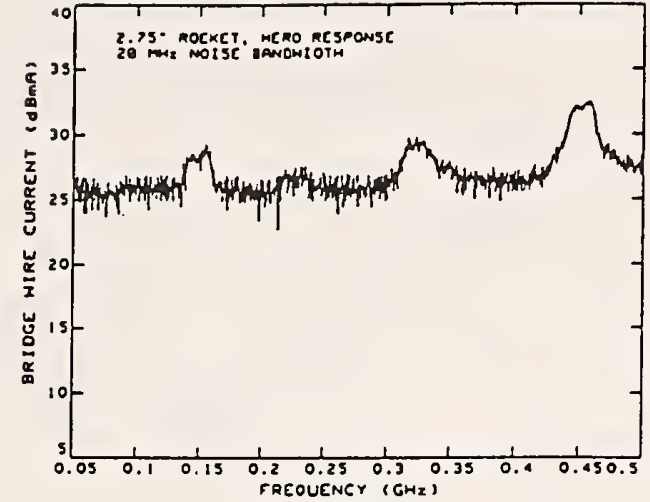

(d)

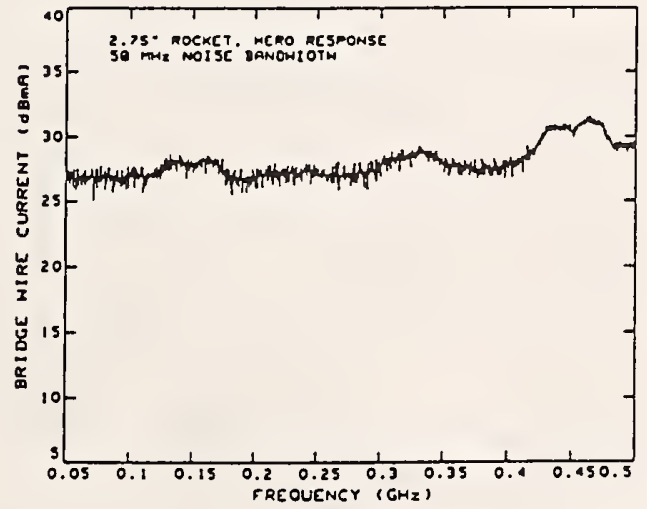

(e)

Figure 4-25. Folding-fin aircraft rocket response -0.05 to $0.5 \mathrm{GHz}$ data. 


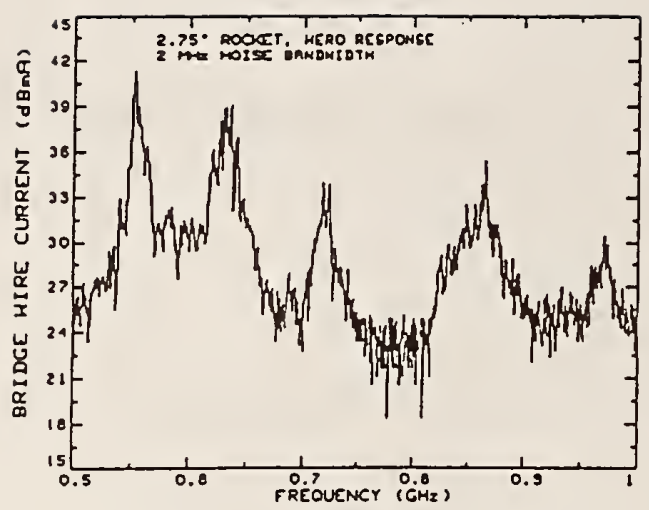

(a)

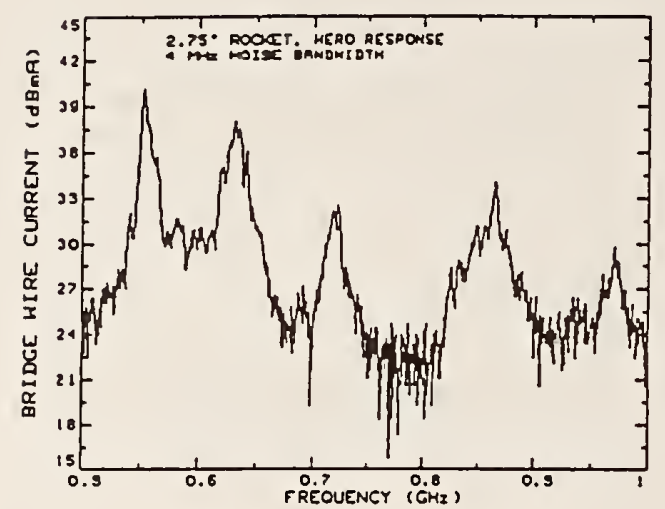

(b)

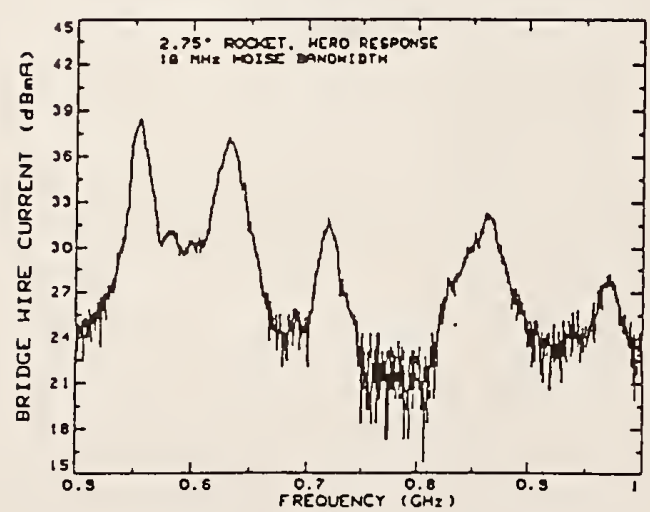

(c)

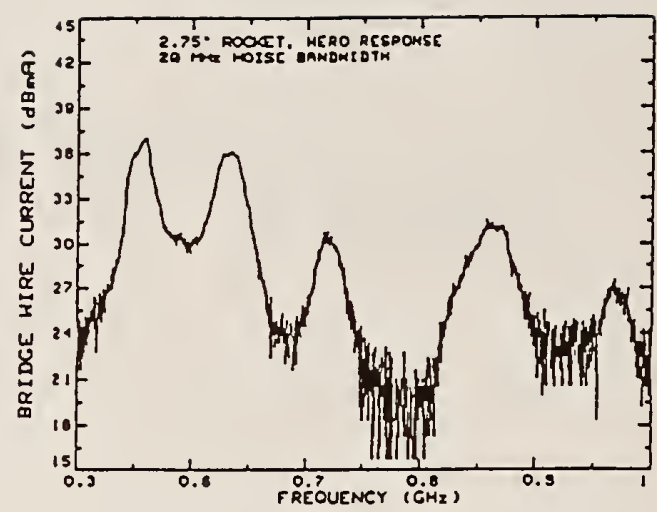

(d)

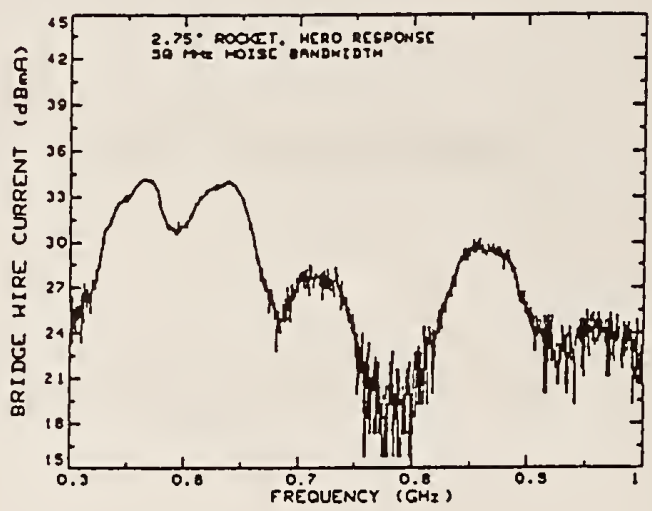

(e)

Figure 4-26. Folding-fin aircraft rocket response -0.5 to $1 \mathrm{GHz}$ data. 


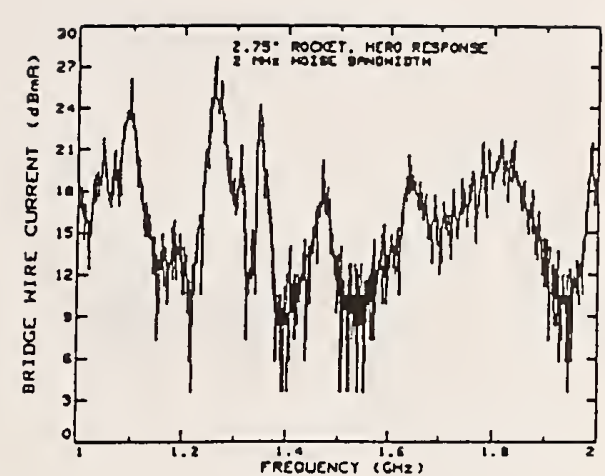

(a)

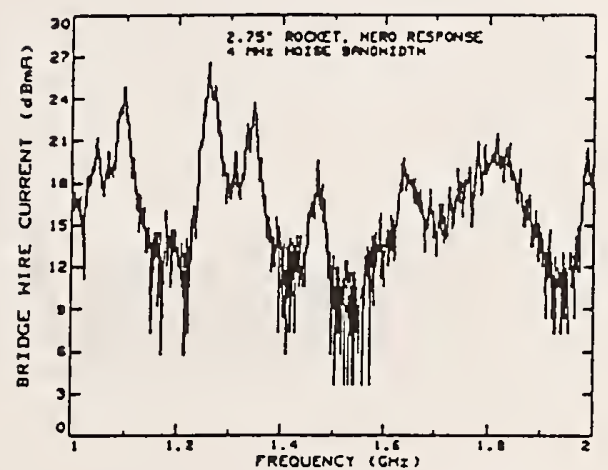

(b)

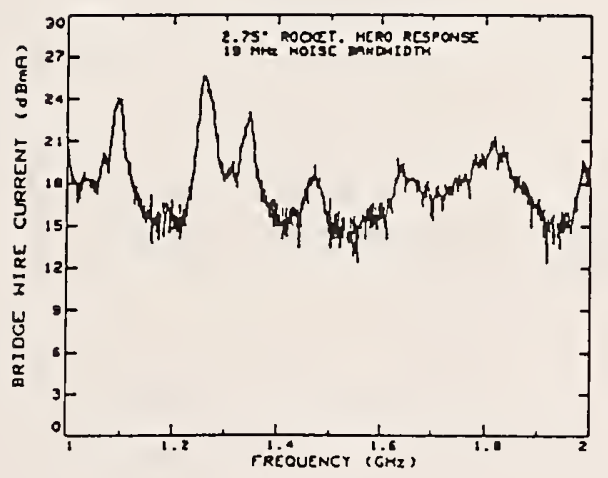

(c)

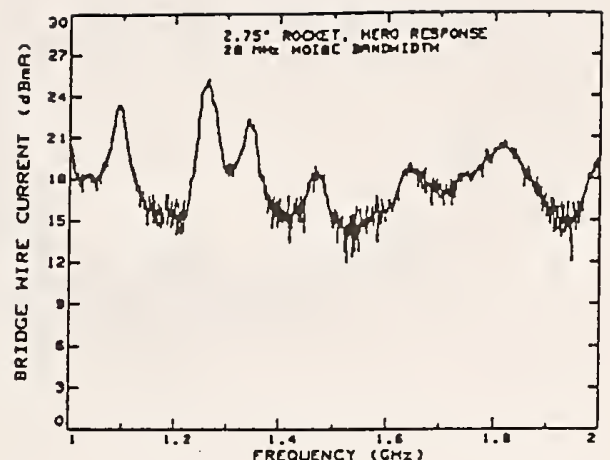

(d)

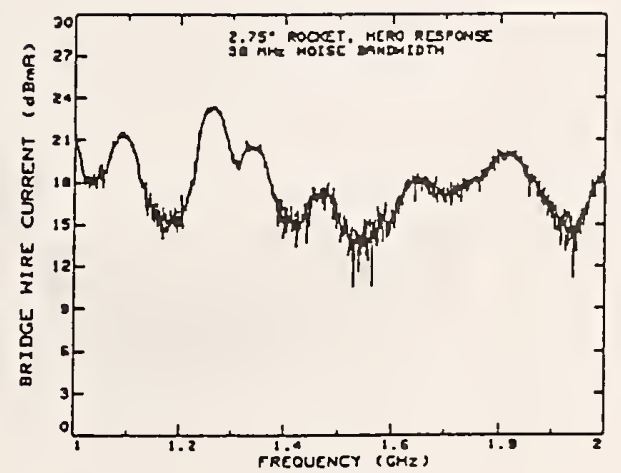

(e)

Figure 4-27. Folding-fin aircraft rocket response - 1 to $2 \mathrm{GHz}$ data. 


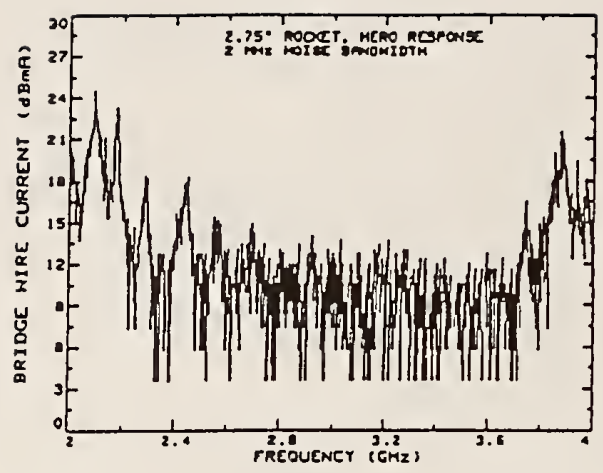

(a)

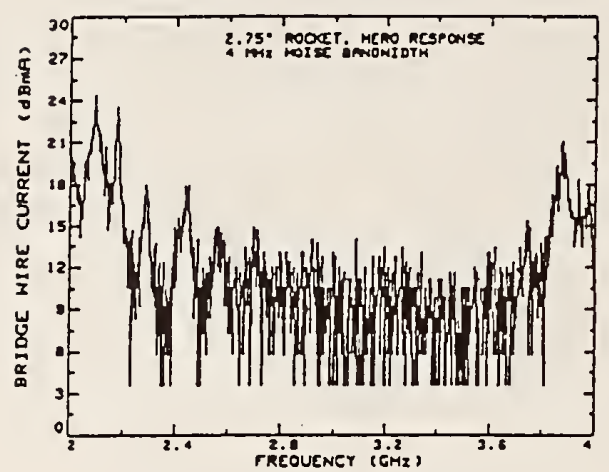

(b)

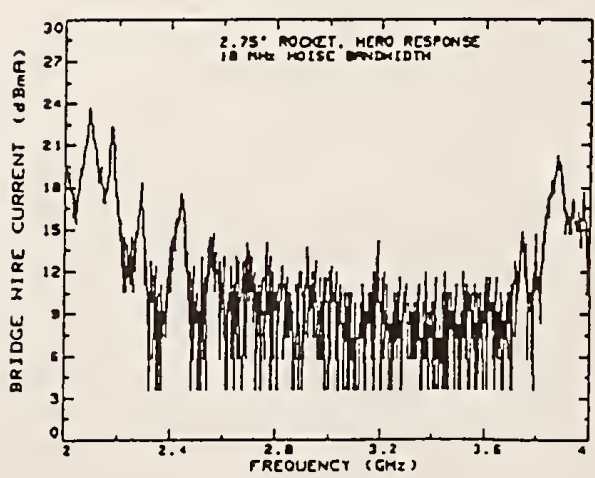

(c)

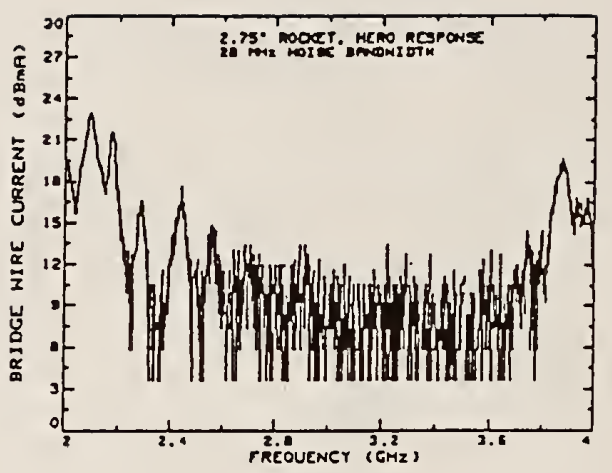

(d)

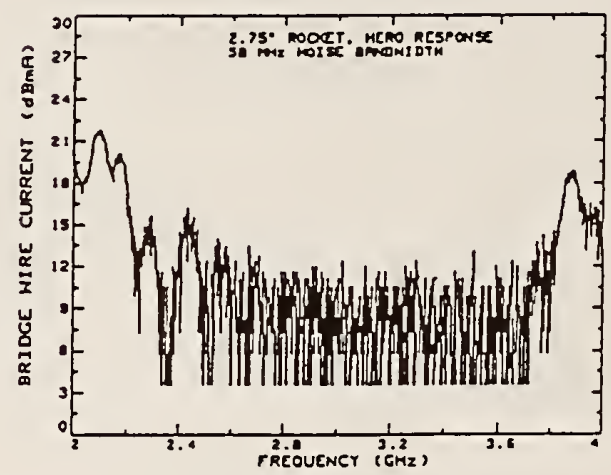

(e)

Figure 4-28. Folding-fin aircraft rocket response - 2 to $4 \mathrm{GHz}$ data. 


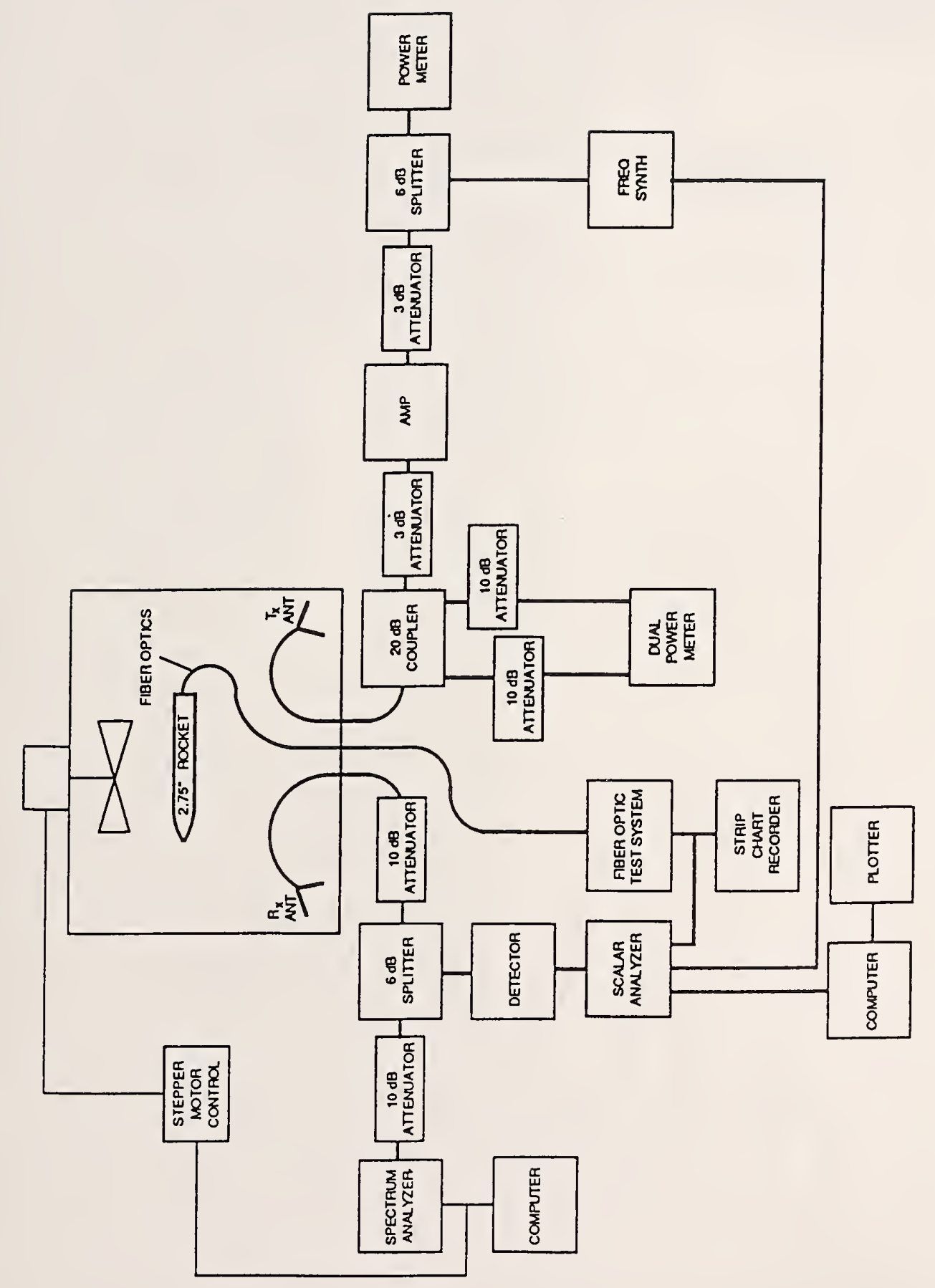

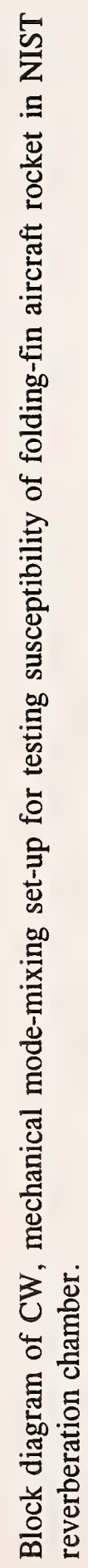

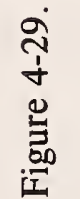




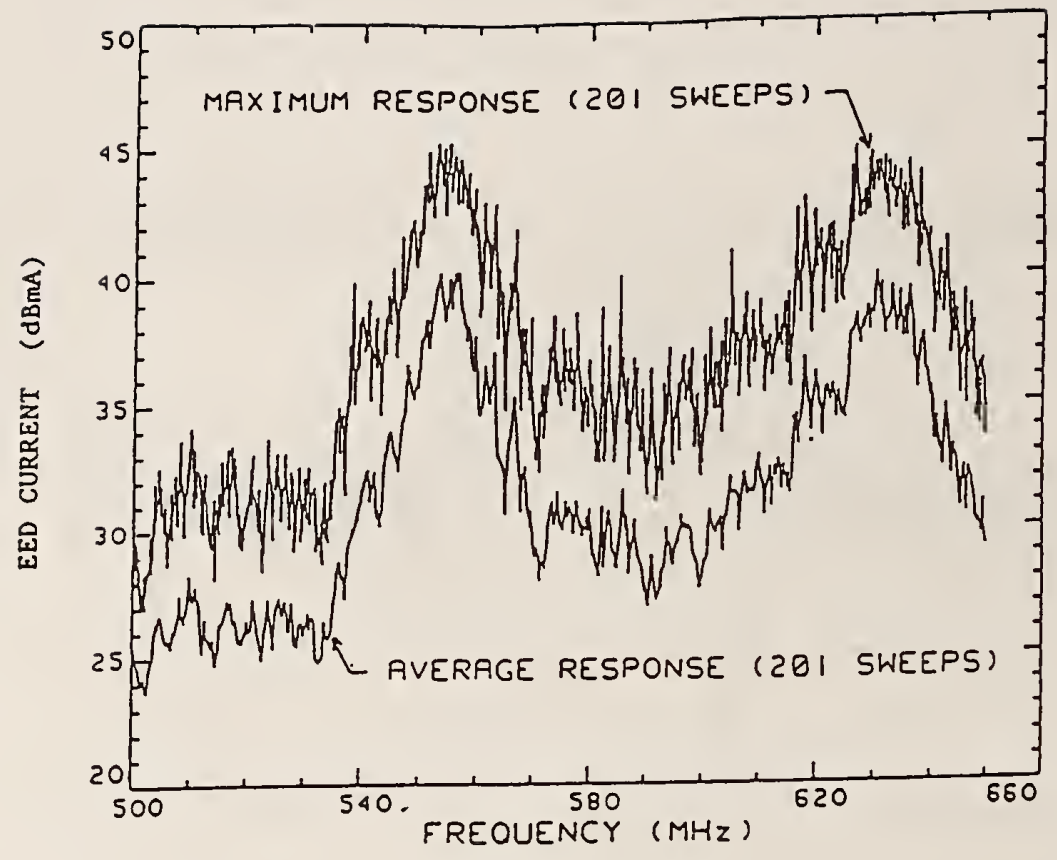

Figure 4-30. Response of folding-fin aircraft rocket to $\mathrm{CW}$ excitation with mechanical mode mixing.

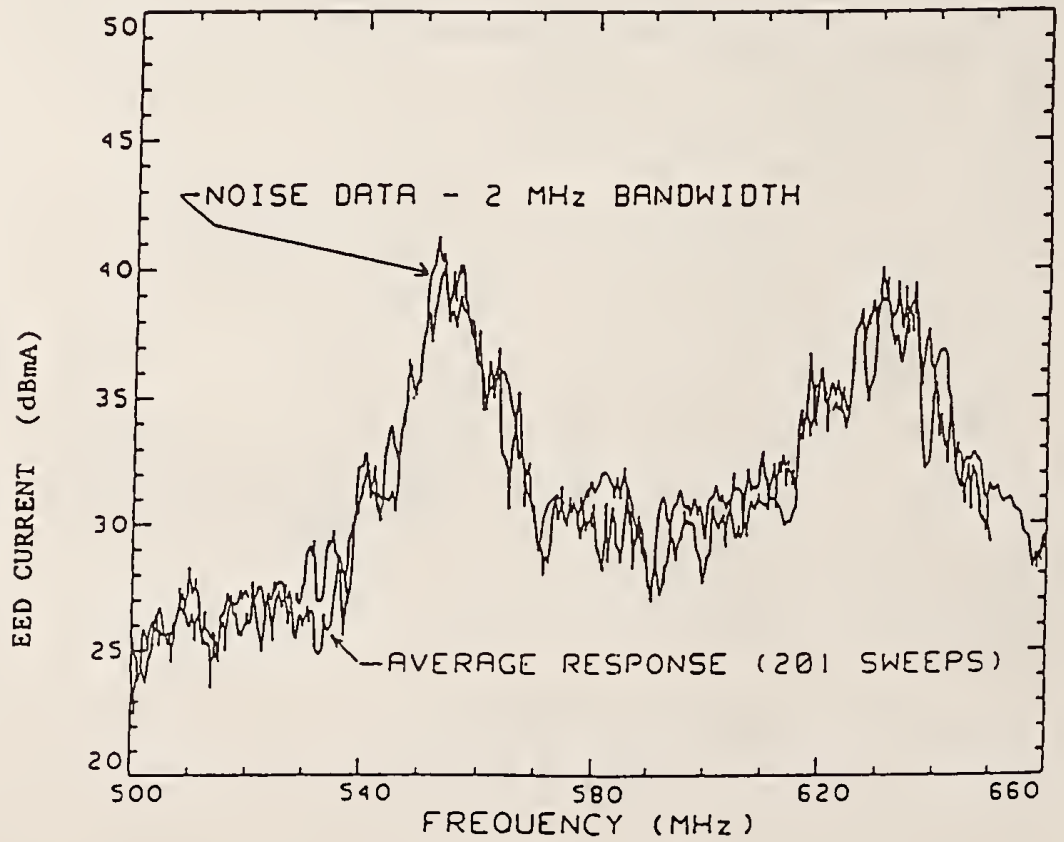

Figure 4-31. Average CW response of folding-fin aircraft rocket vs. noise response (2 $\mathrm{MHz}$ bandwidth). 


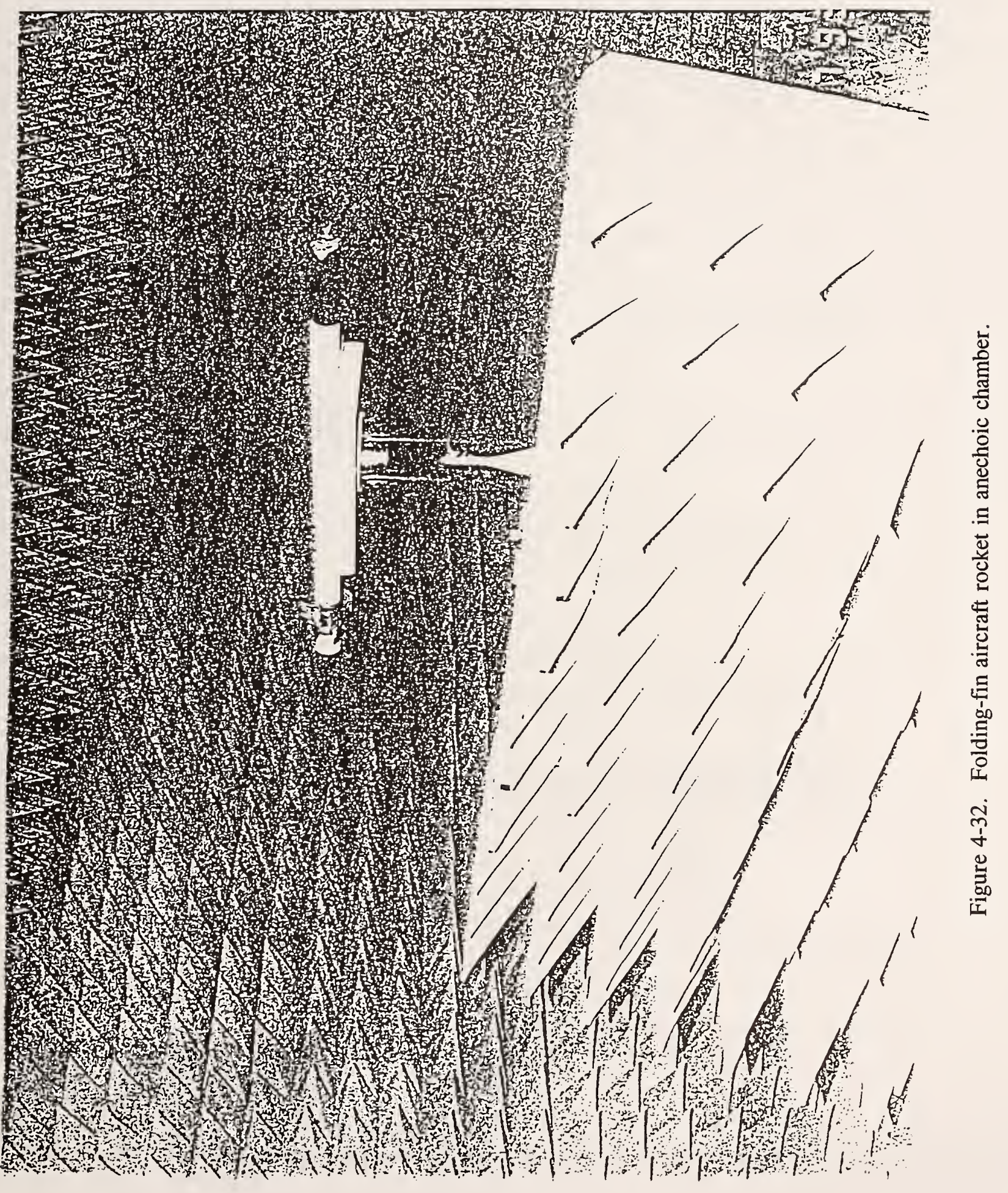



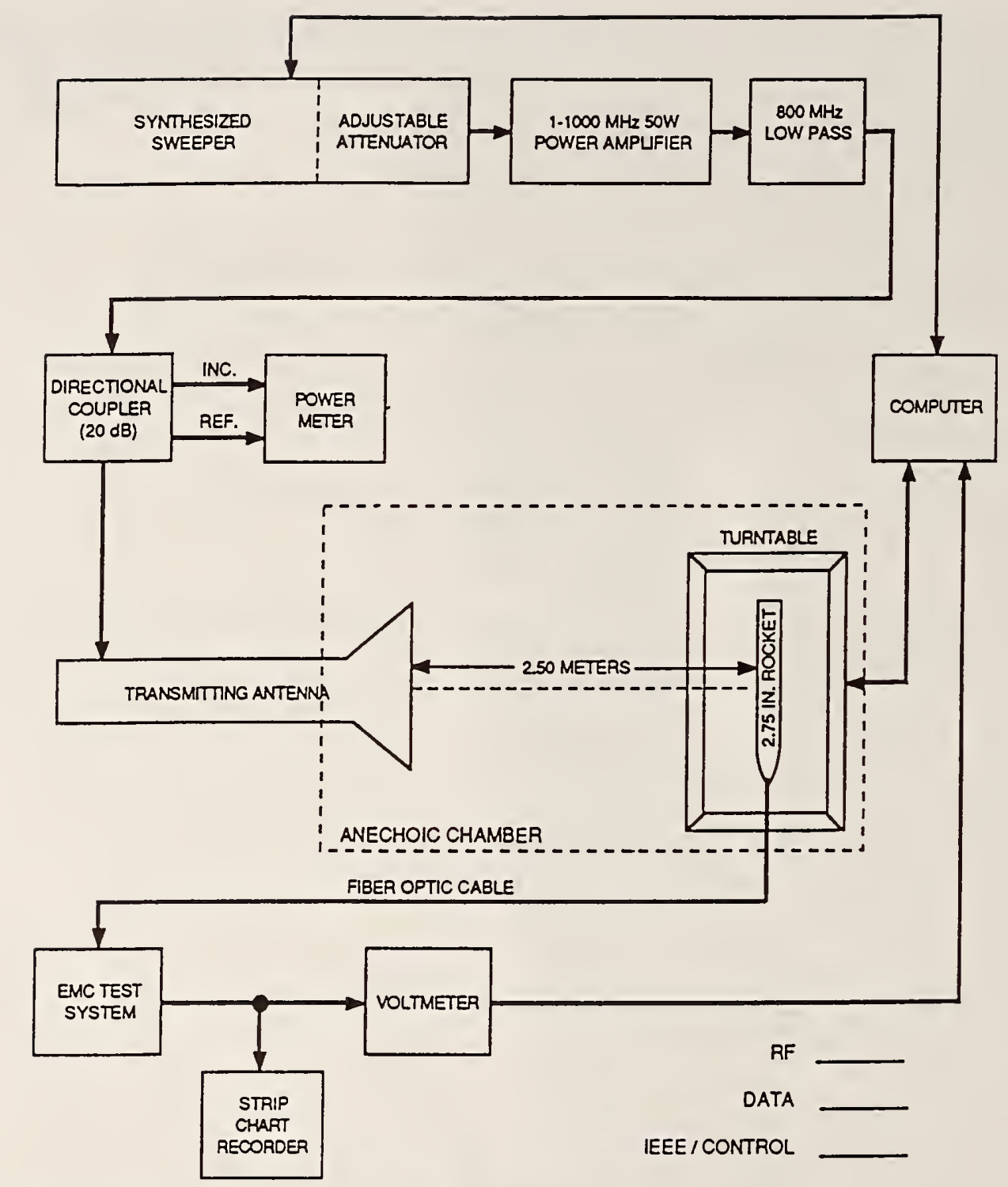

TEST ORIENTATION

$0-180$ DEG. AZIMUTH

$0,45,90,135,180$ DEG. ROLL

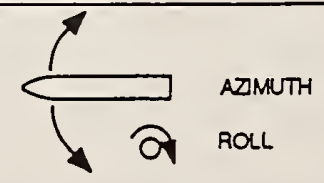

Figure 4-33. Diagram of anechoic chamber discrete frequency set-up. 


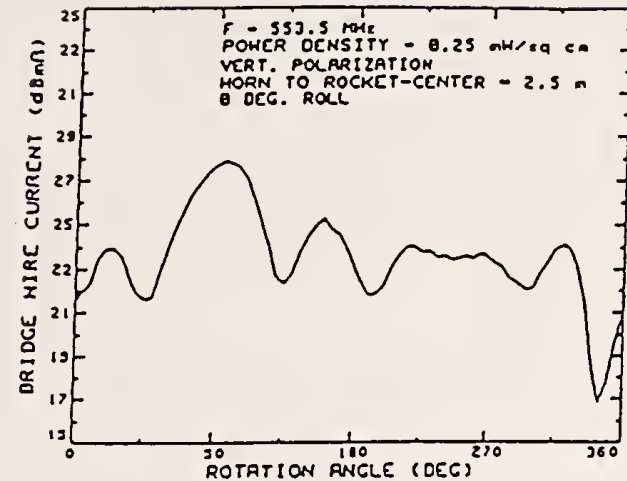

(a)

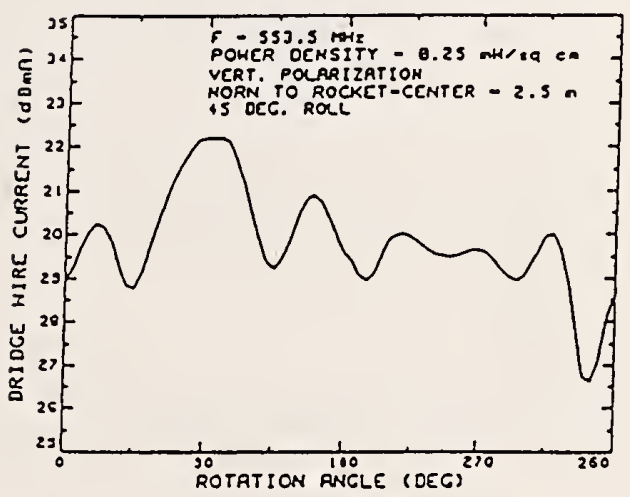

(b)

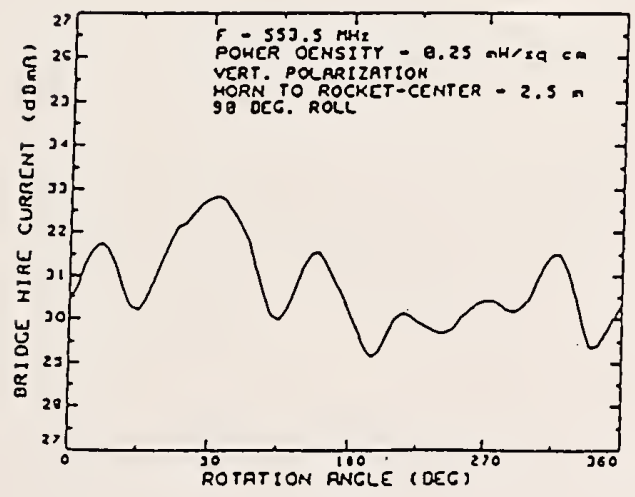

(c)

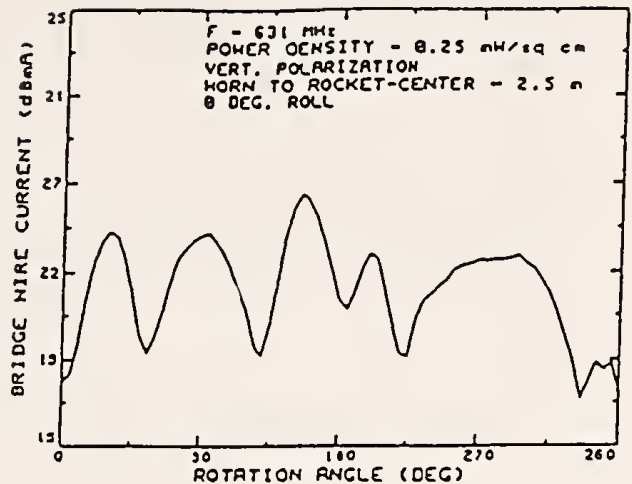

(d)

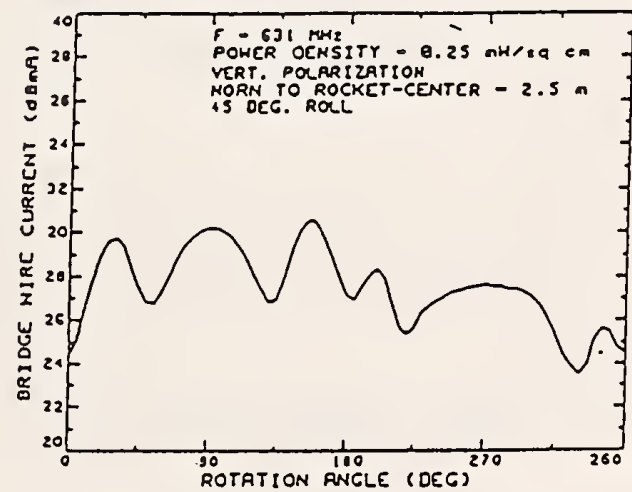

(e)

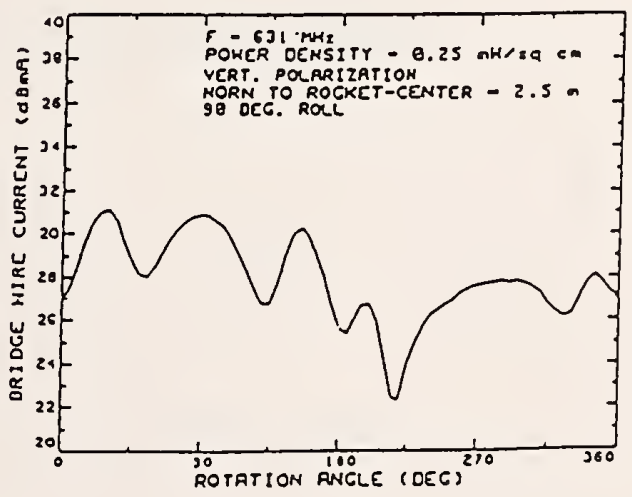

Figure 4-34. Folding-fin aircraft rocket response to vertically polarized illumination at $553.5 \mathrm{MHz}$ and $631 \mathrm{MHz}$. 


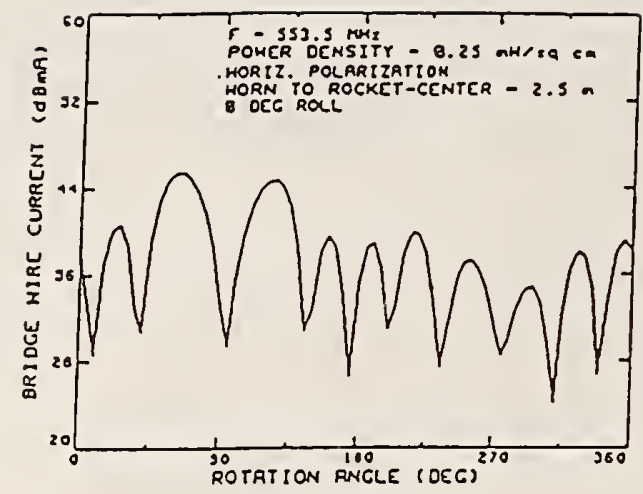

(a)

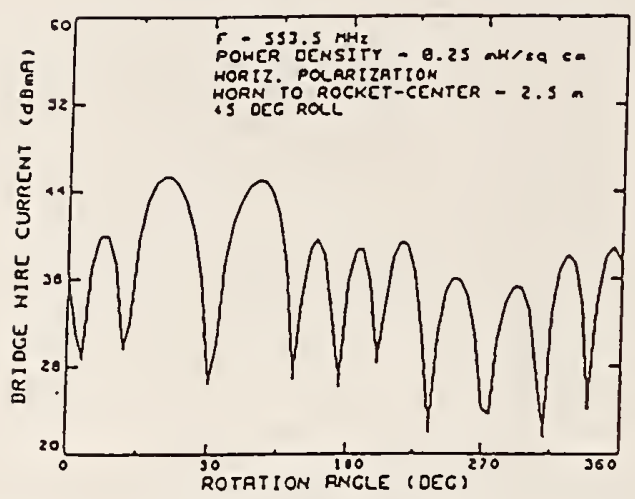

(b)

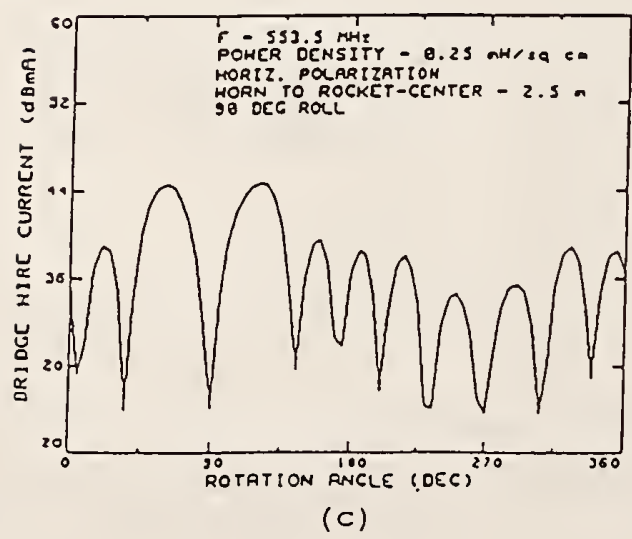

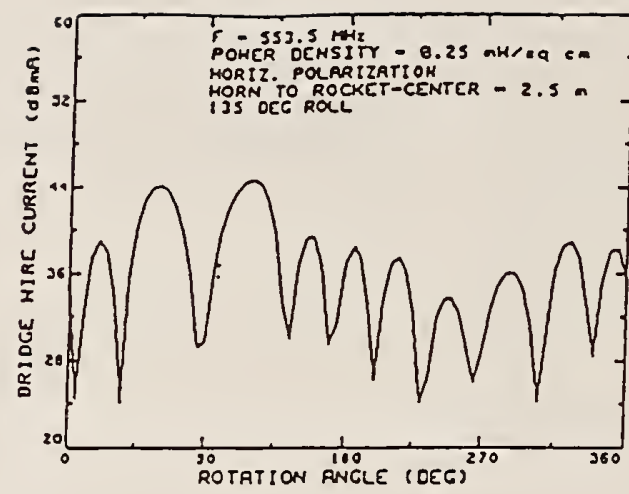

(d)

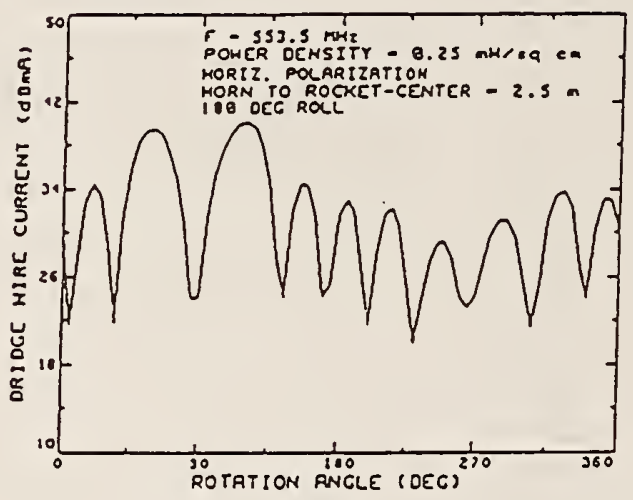

(e)

Figure 4-35. Folding-fin aircraft rocket response to horizontally polarized illumination at $553.5 \mathrm{MHz}$. 


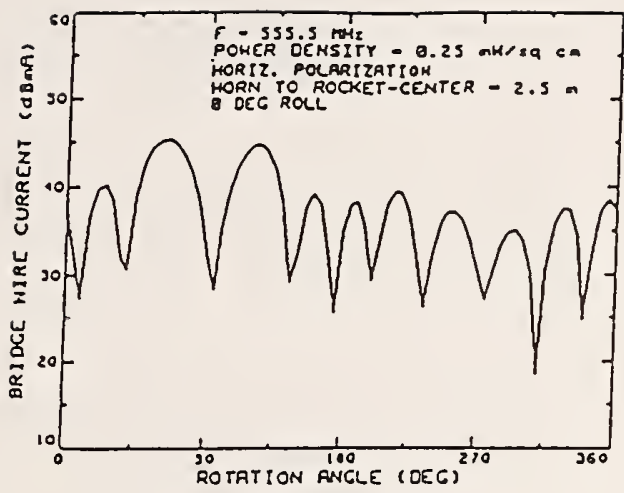

(a)

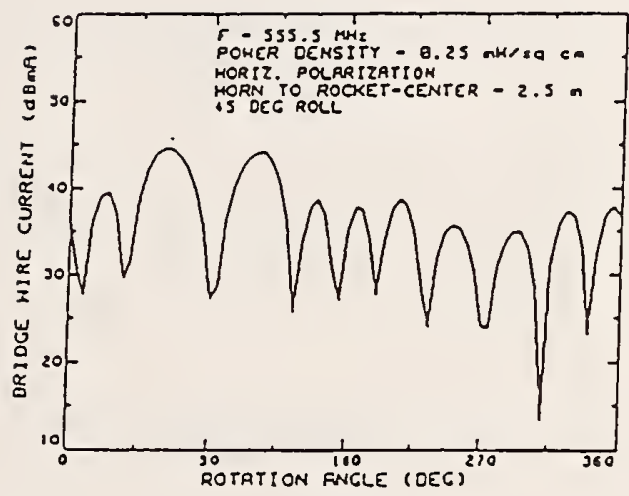

(b)

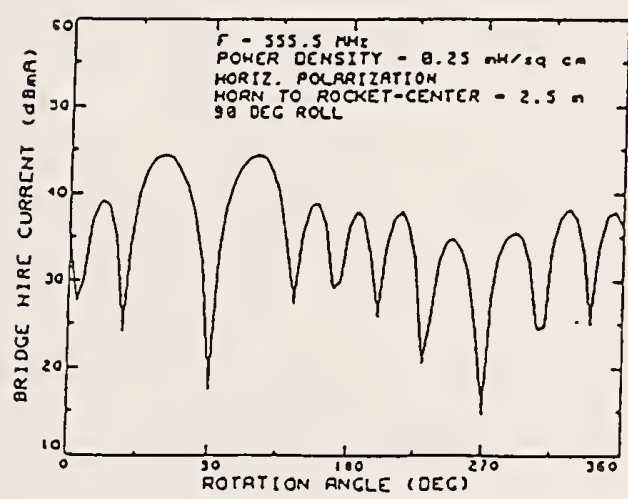

(c)

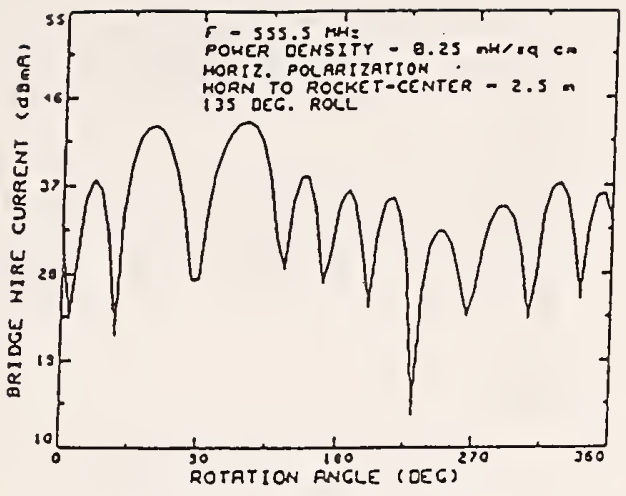

(d)

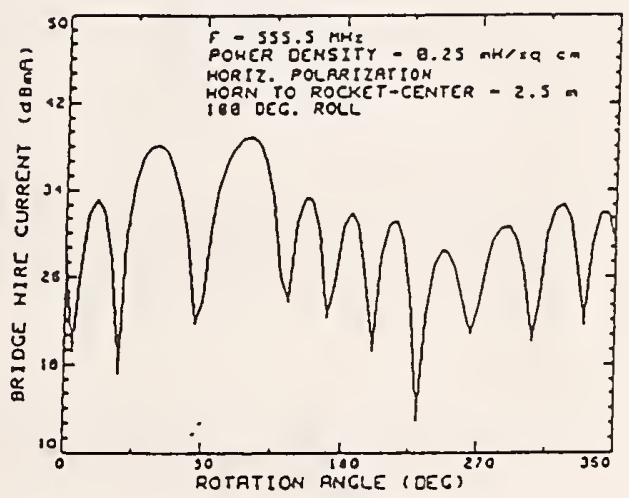

(e)

Figure 4-36. Folding-fin aircraft rocket response to horizontally polarized illumination at $555.5 \mathrm{MHz}$. 


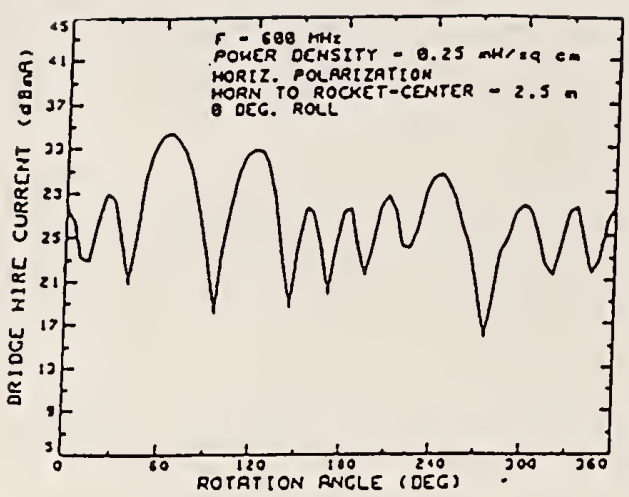

(a)

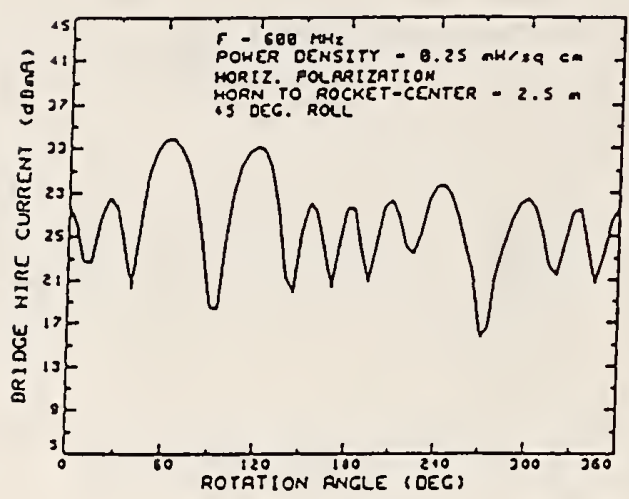

(b)

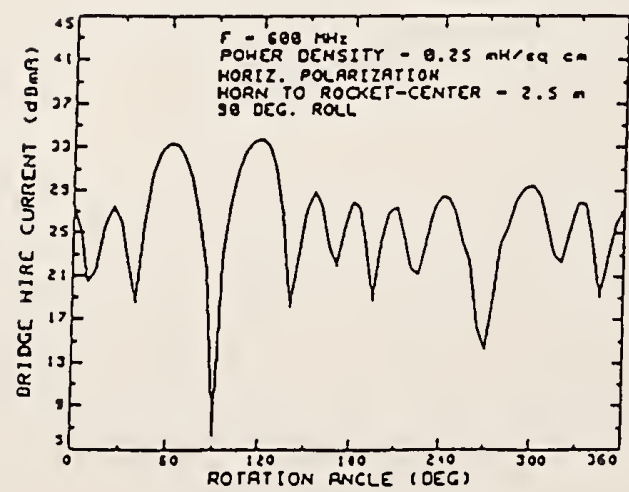

(c)

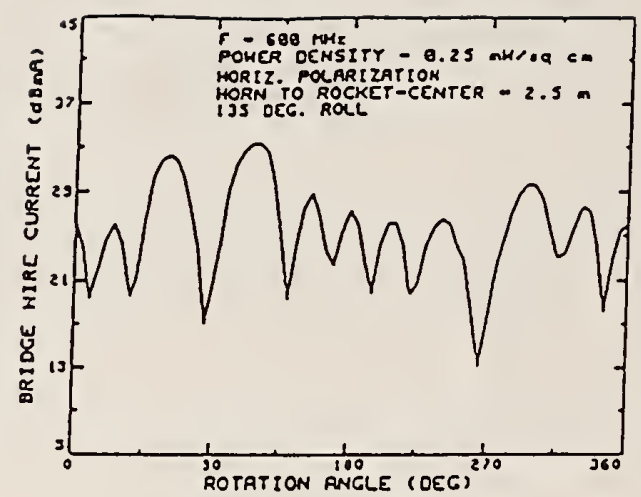

(d)

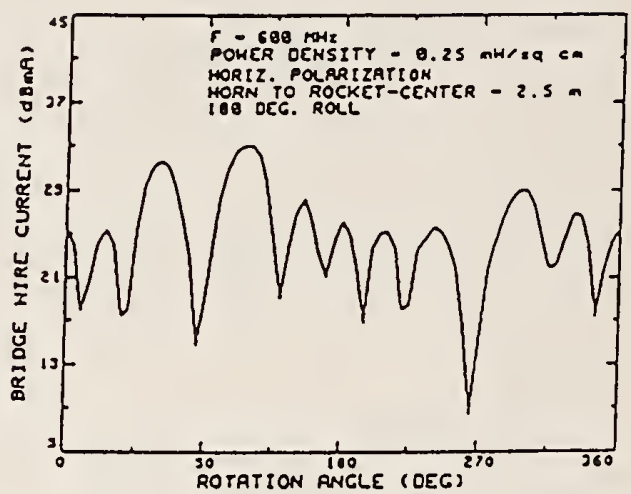

(e)

Figure 4-37. Folding-fin aircraft rocket response to horizontally polarized illumination at $600 \mathrm{MHz}$. 


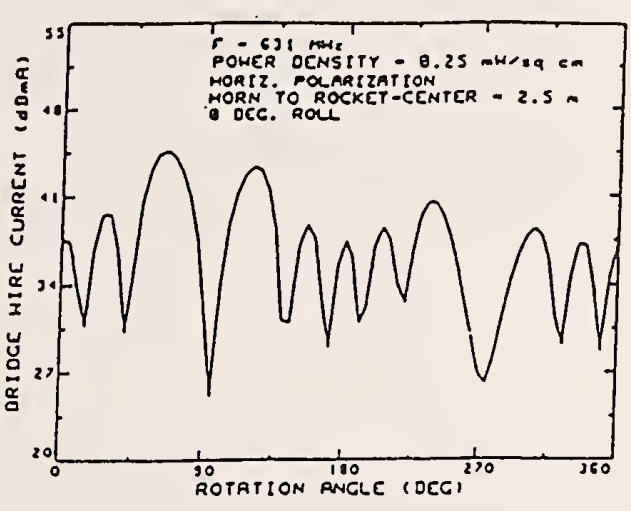

(a)

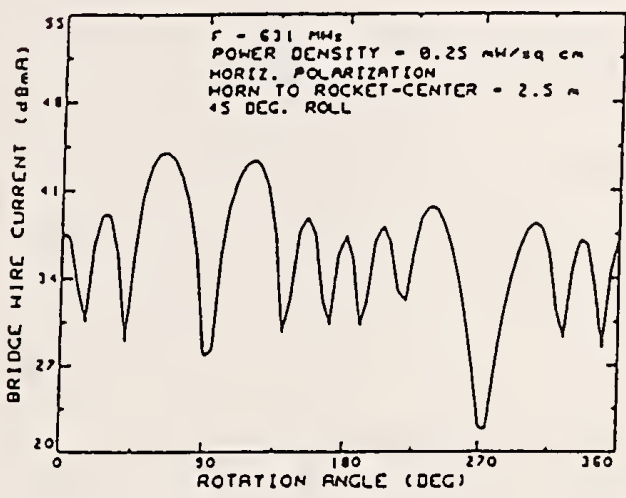

(b)

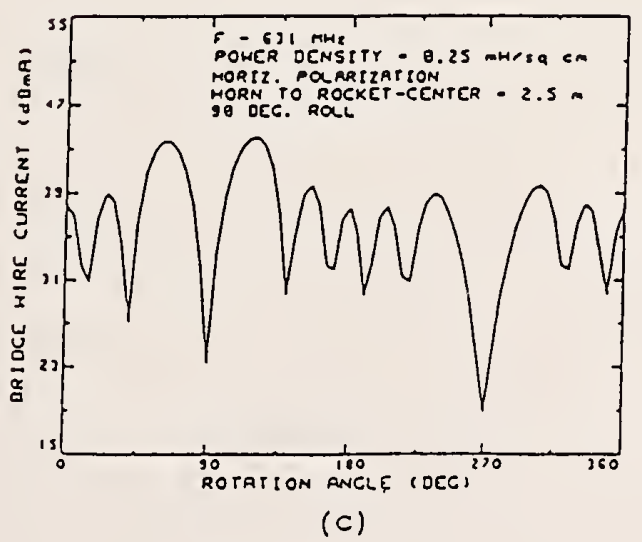

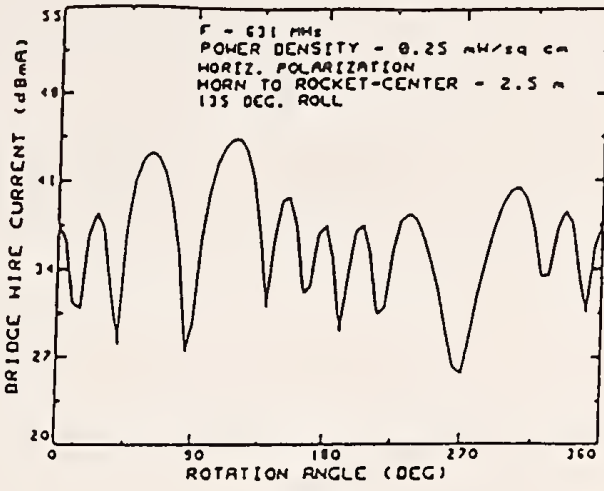

(d)

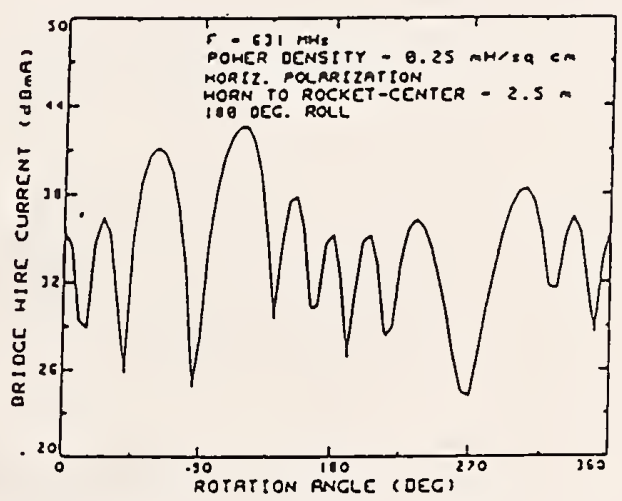

(e)

Figure 4-38. Folding-fin aircraft rocket response to horizontally polarized illumination at $631 \mathrm{MHz}$. 

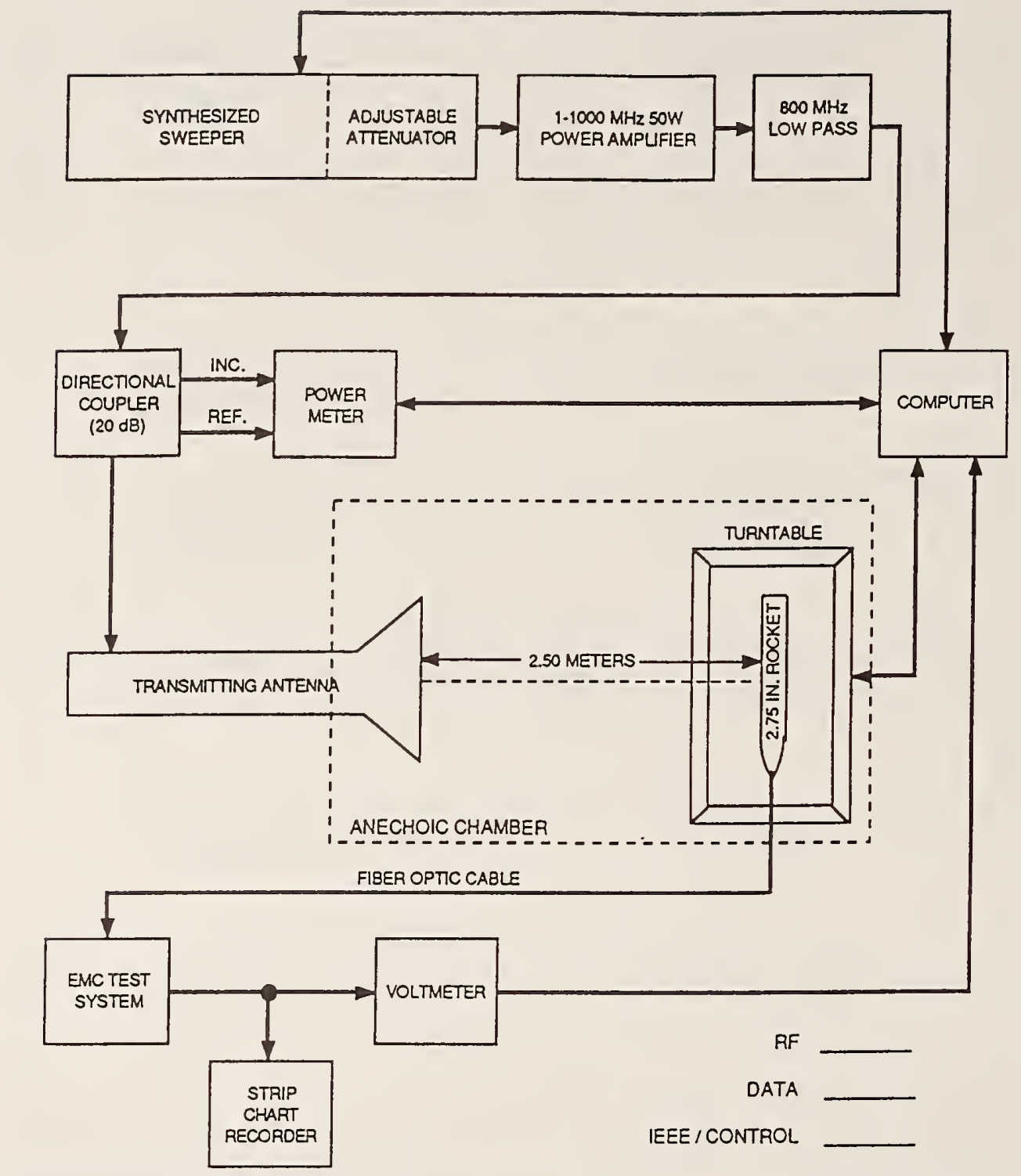

TEST ORIENTATION

0-180 DEG. AZIMUTH

$0,45,90,135,180$ DEG. ROLL

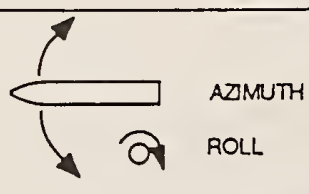

Figure 4-39. Diagram of anechoic chamber swept frequency set-up. 


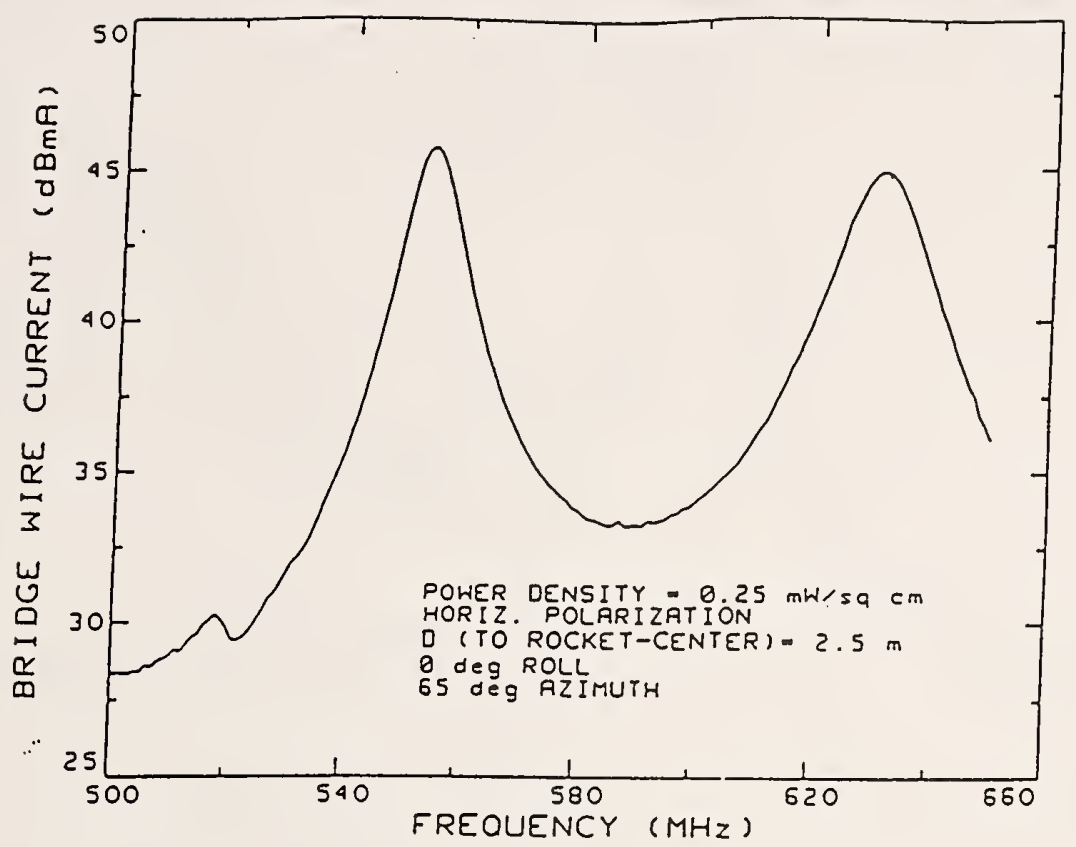

Figure 4-40. Anechoic chamber response of folding-fin aircraft rocket.

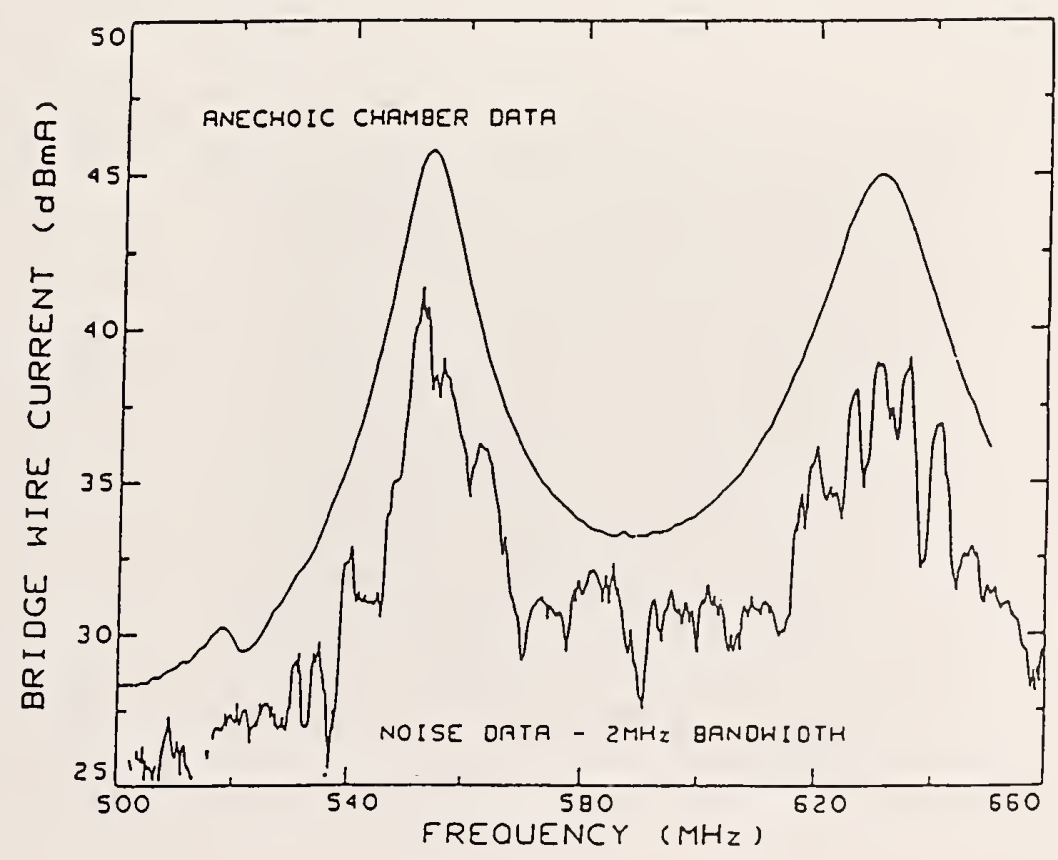

Figure 4-41. Folding-fin aircraft rocket anechoic chamber data vs. reverberation chamber $2 \mathrm{MHz}$ noise data. 


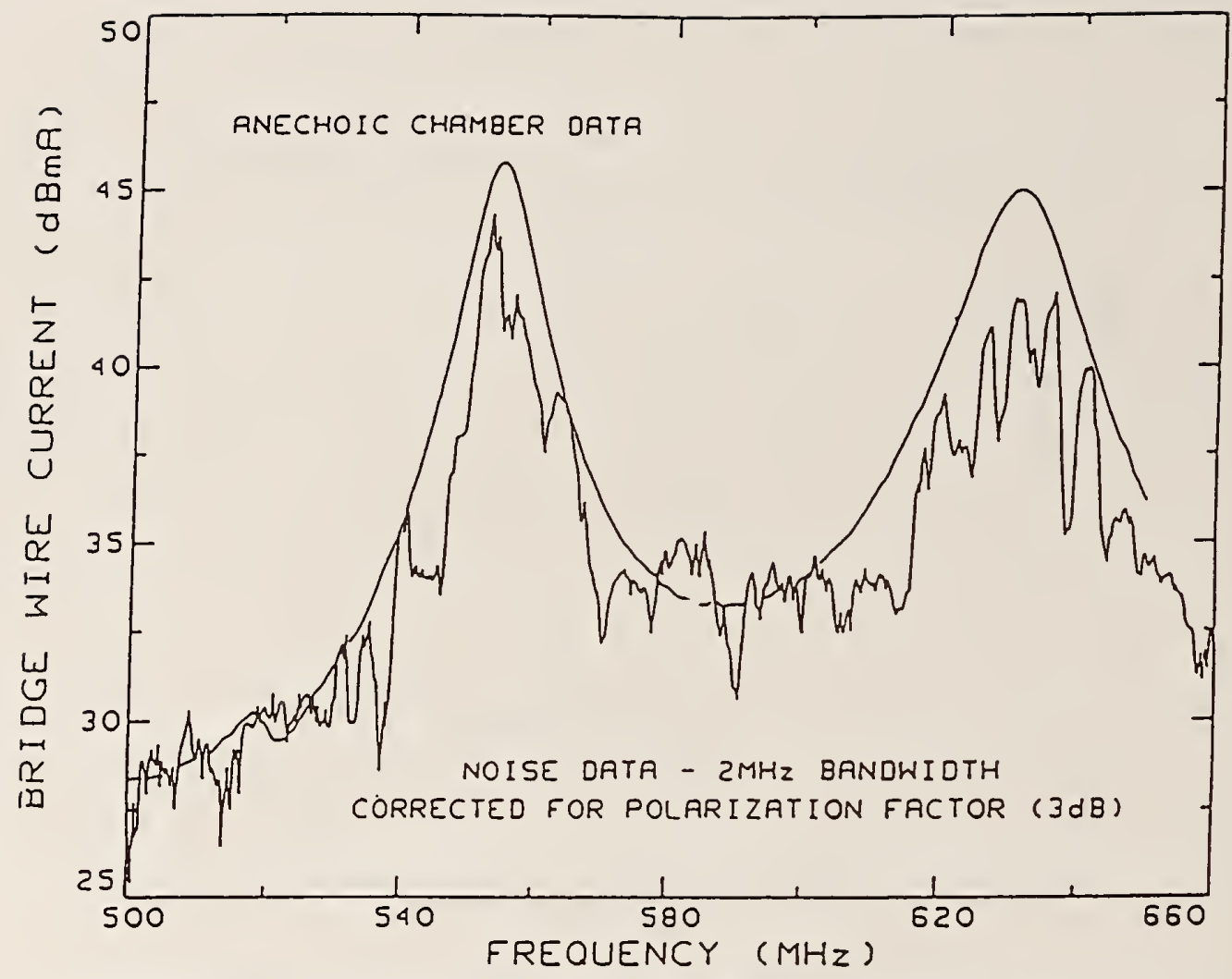

Figure 4-42. Folding-fin aircraft rocket anechoic chamber data vs. reverberation chamber $2 \mathrm{MHz}$ noise data corrected for polarization factor. 


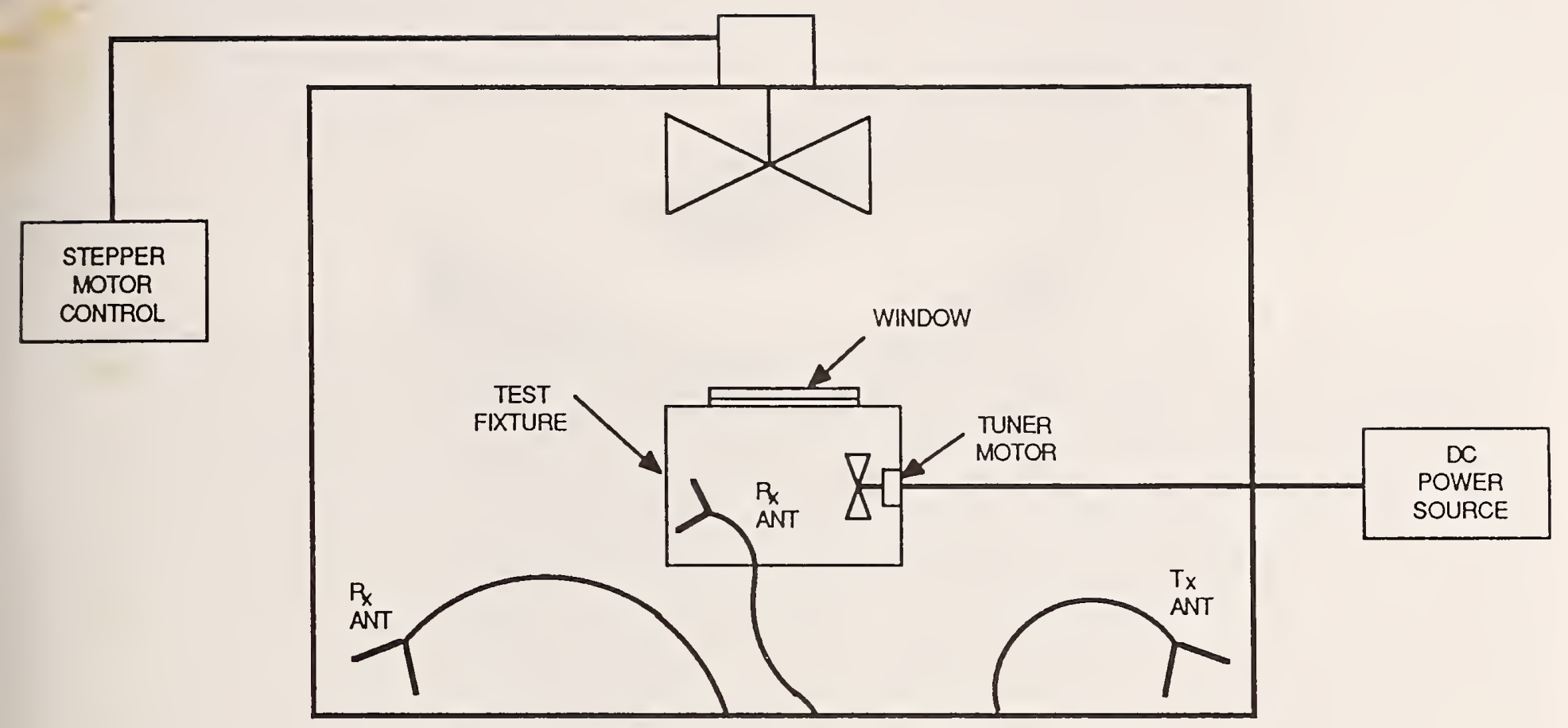

Figure 4-43. "NESTED" chamber technique for collecting SE data.

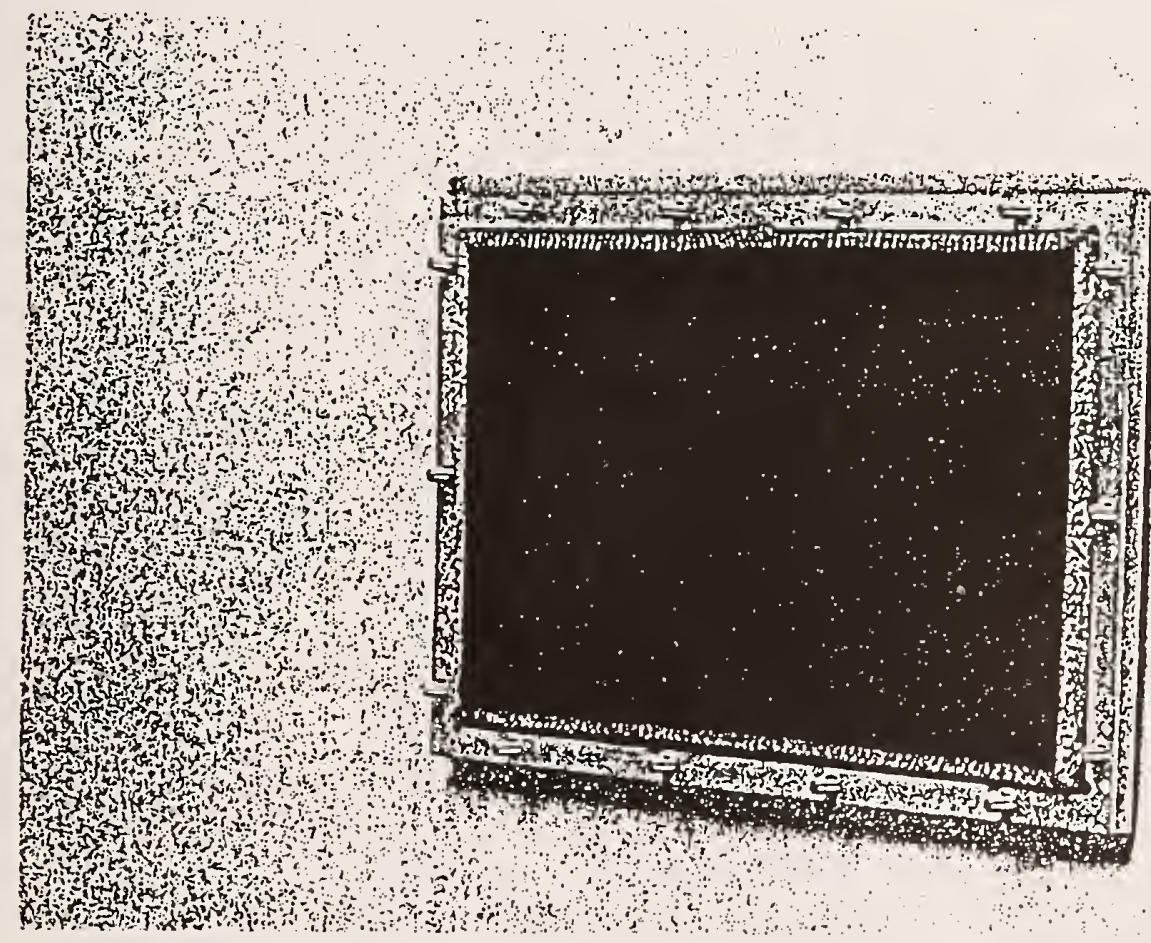

Figure 4-44. Photo of wire mesh window. 


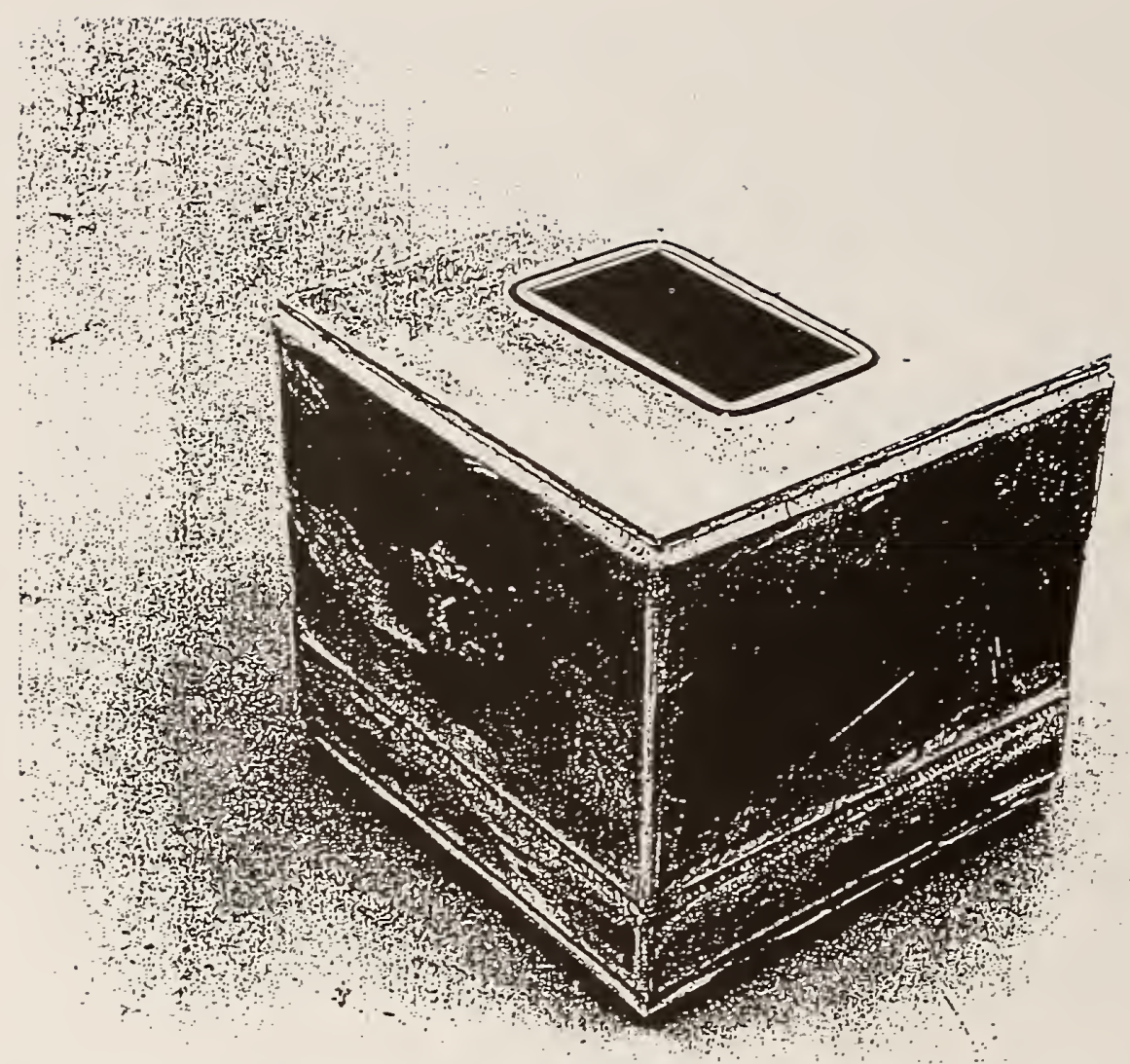

Figure 4-45. Photo of test fixture used for measuring the shielding effectiveness of shielded window using the nested reverberation chamber technique.

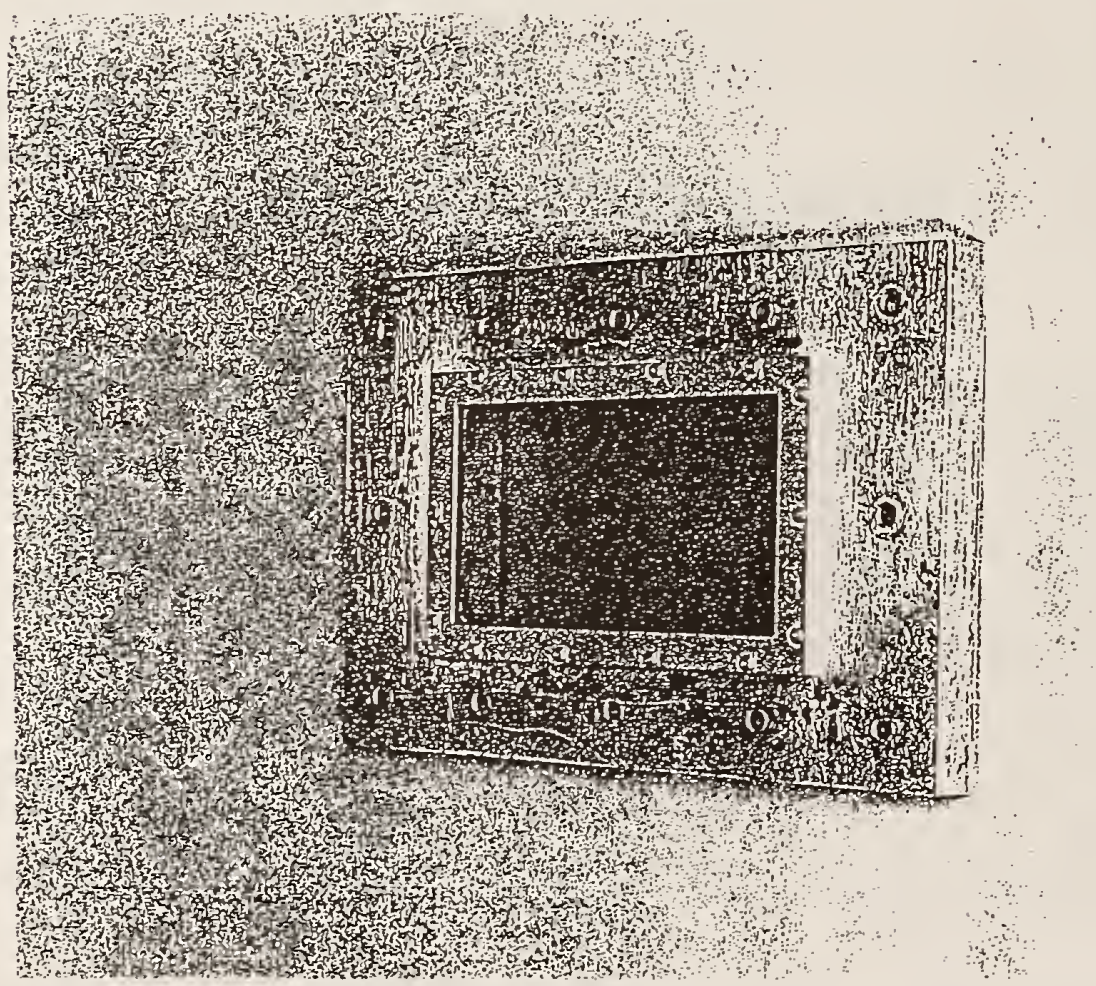

Figure 4-46. Shielded window mounting for test fixture. 


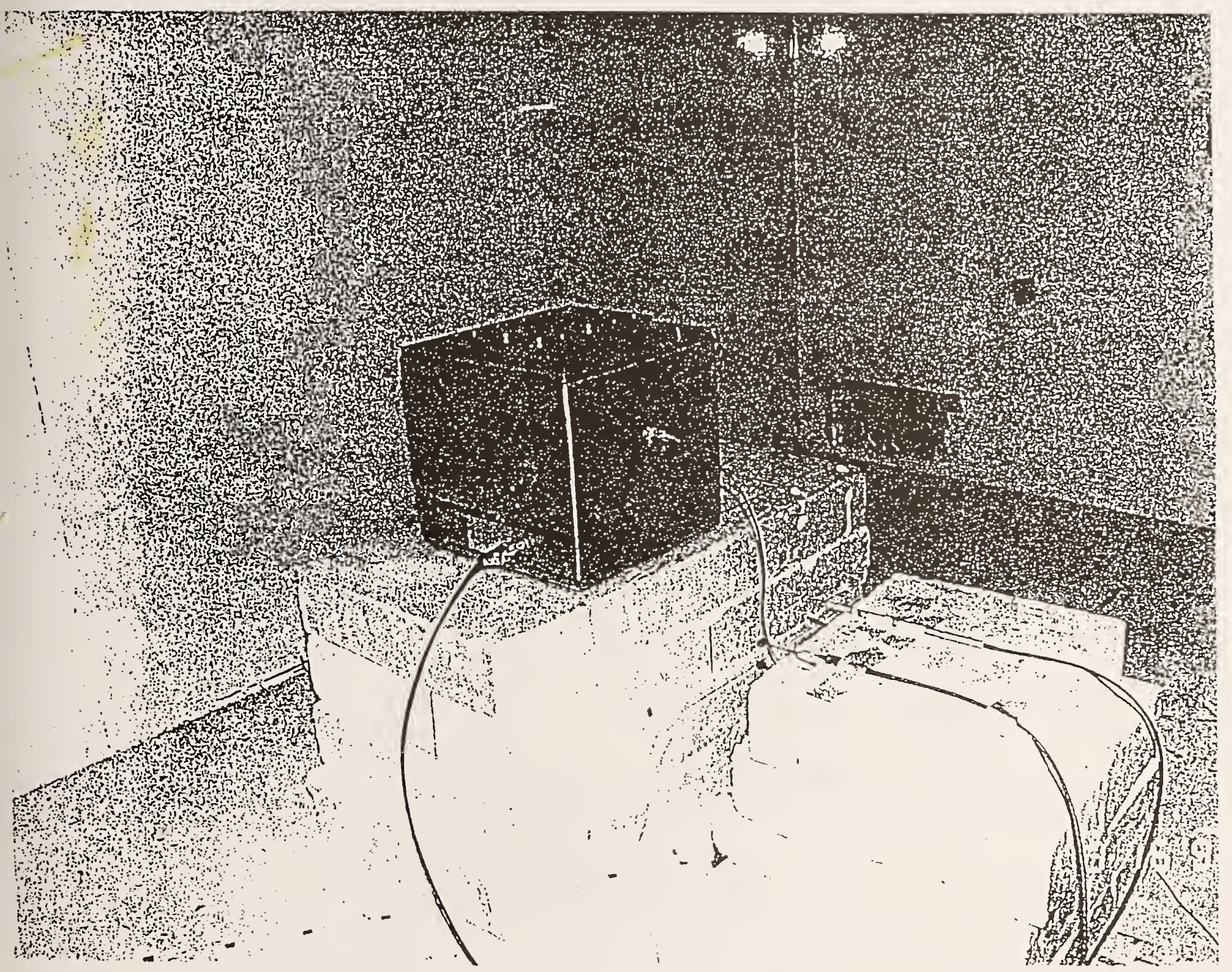

Figure 4-47. Window SE test fixture mounted inside NIST reverberation chamber. 


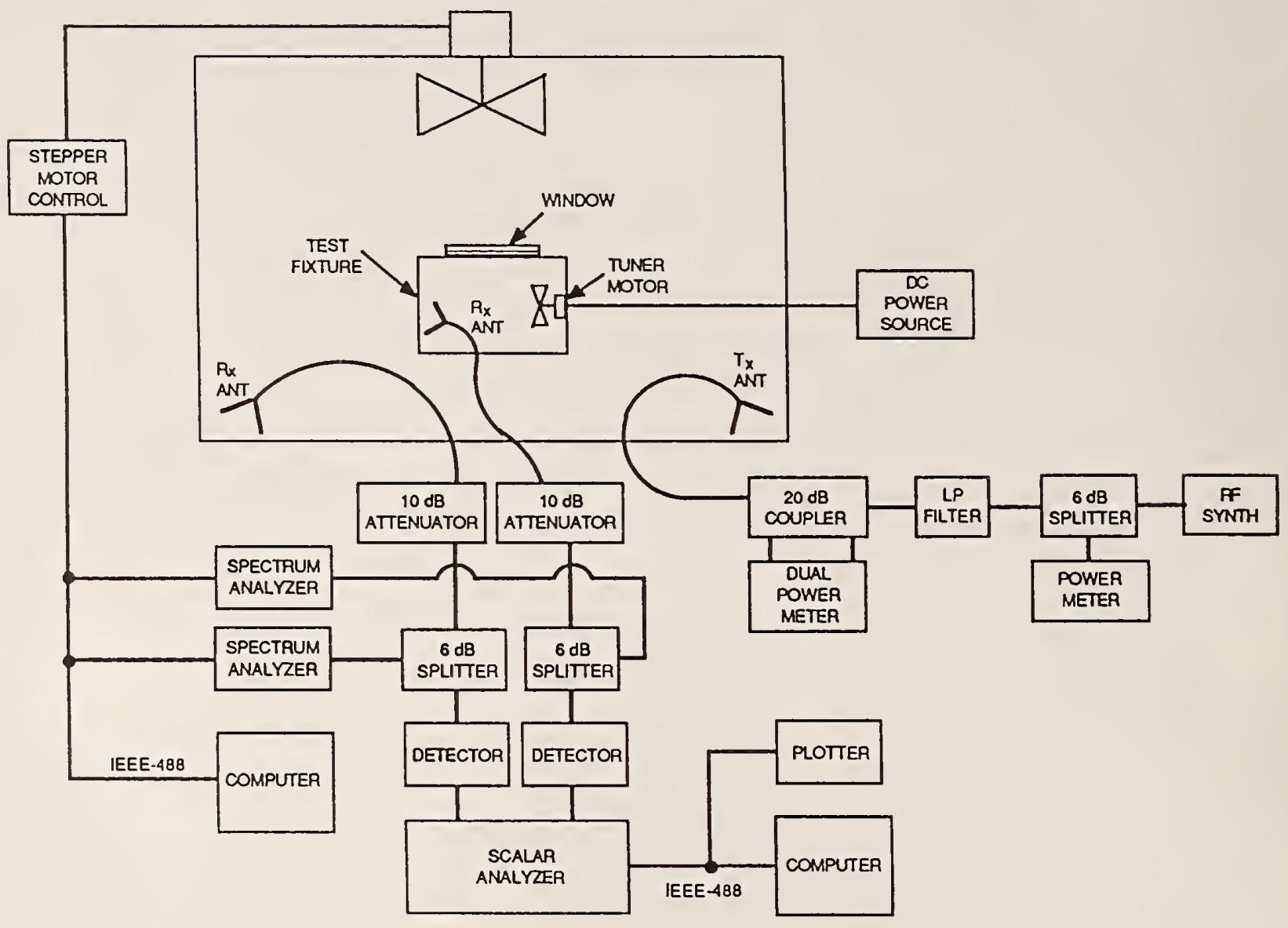

Figure 4-48. Reverberation chamber CW (mechanical mode mixing) test set-up for SE measurements. 


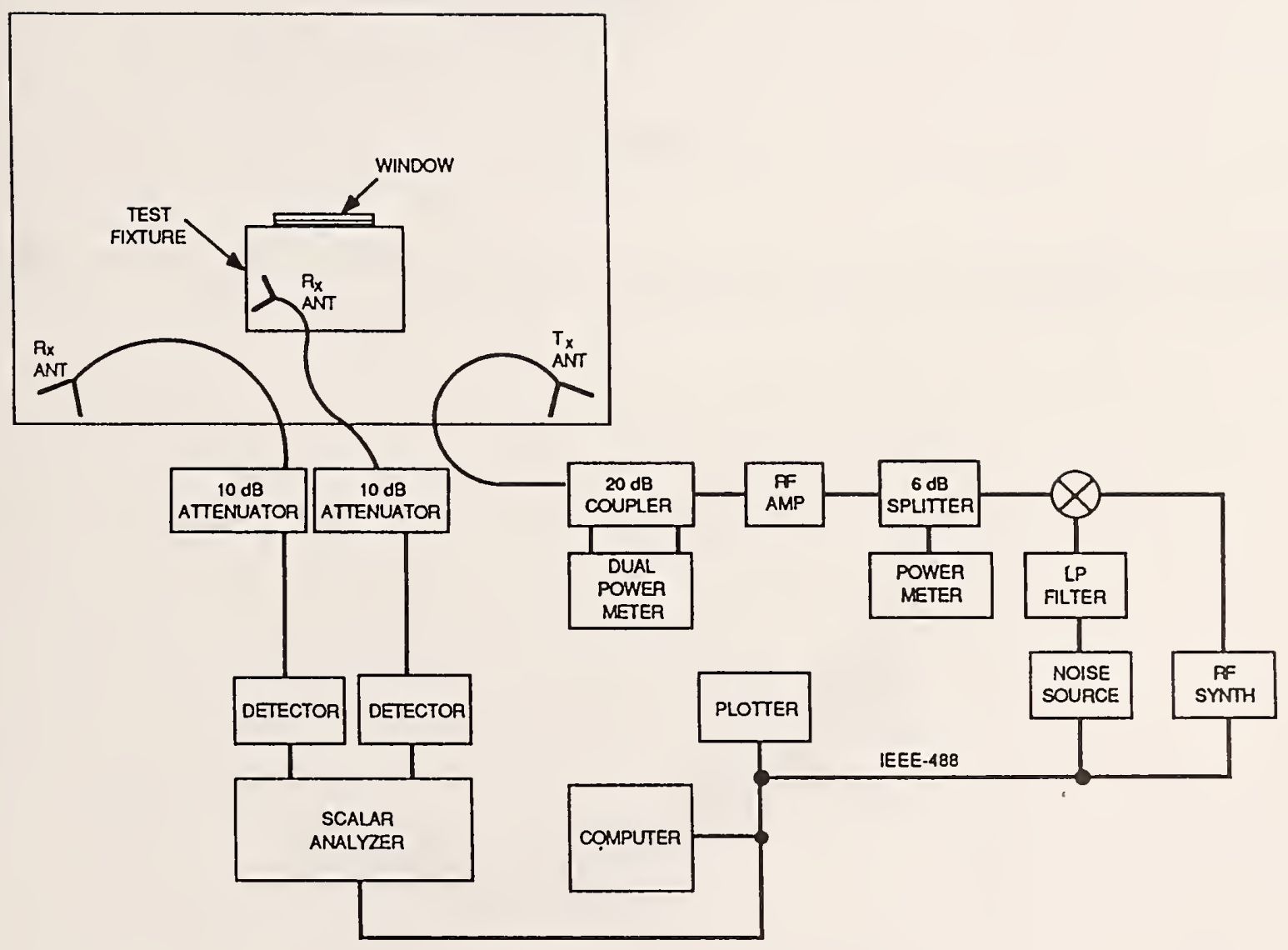

Figure 4-49. Reverberation chamber BLWGN test set-up for SE measurements. 


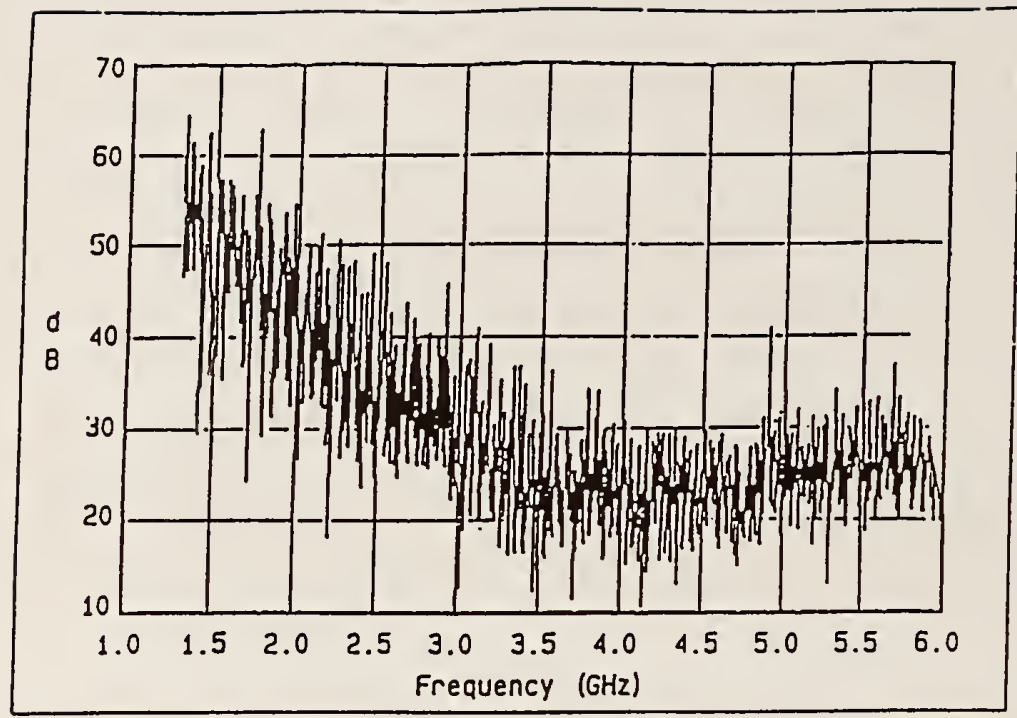

Figure 4-50. Results of window SE measurements made using BLWGN technique with $2 \mathrm{MHz}$ bandwidth.

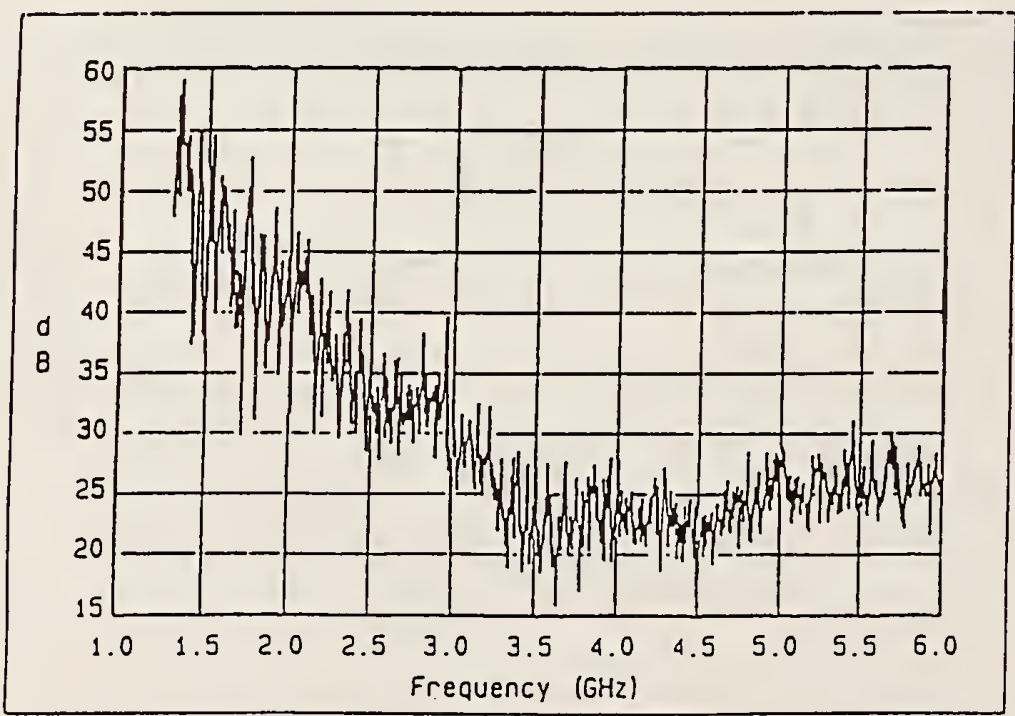

Figure 4-51. Results of window SE measurements made using BLWGN technique with $10 \mathrm{MHz}$ bandwidth. 


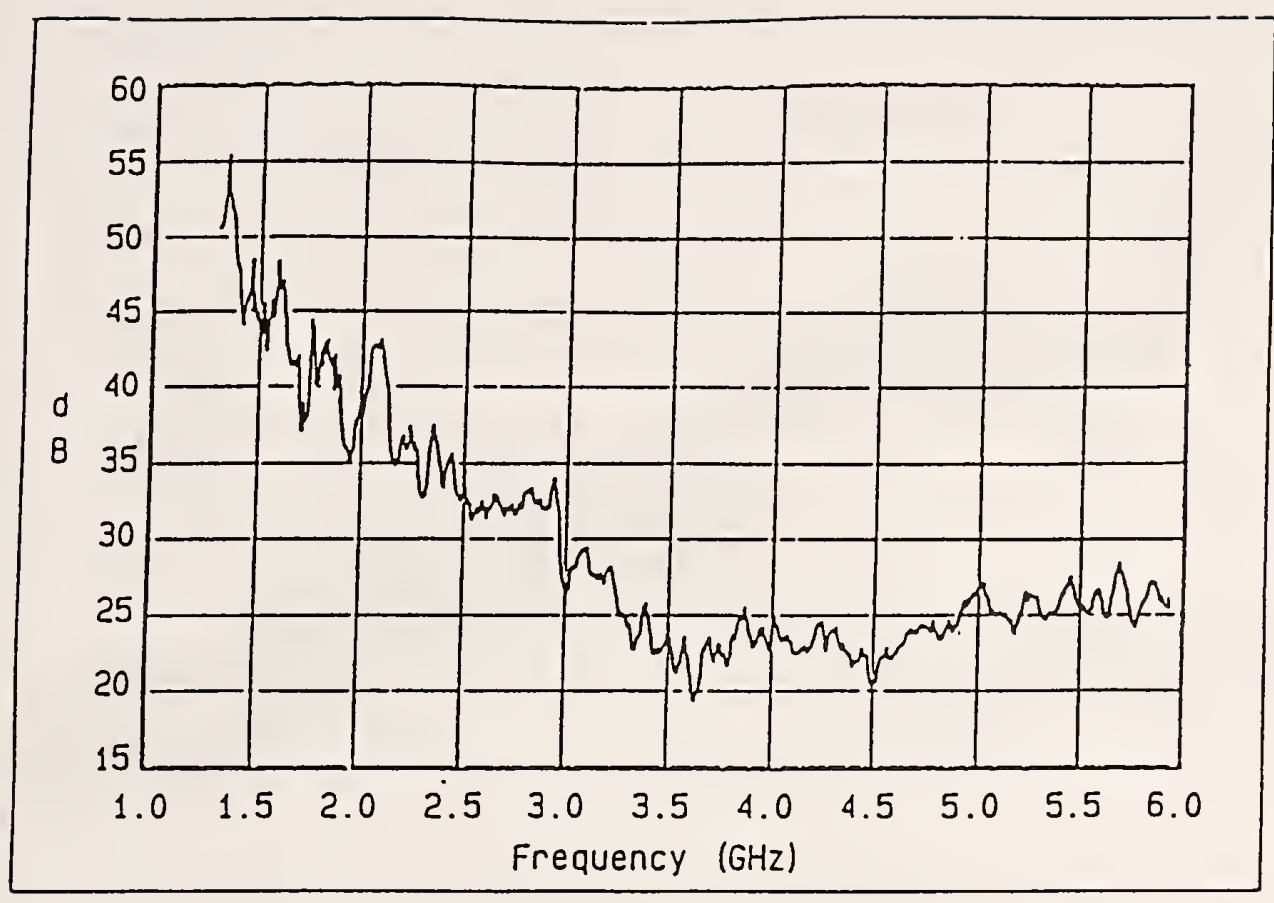

Figure 4-52. Results of window SE measurements made using BLWGN technique with $50 \mathrm{MHz}$ bandwidth. 
CHAMBER VS TEST FIXTURE (BASELINES)

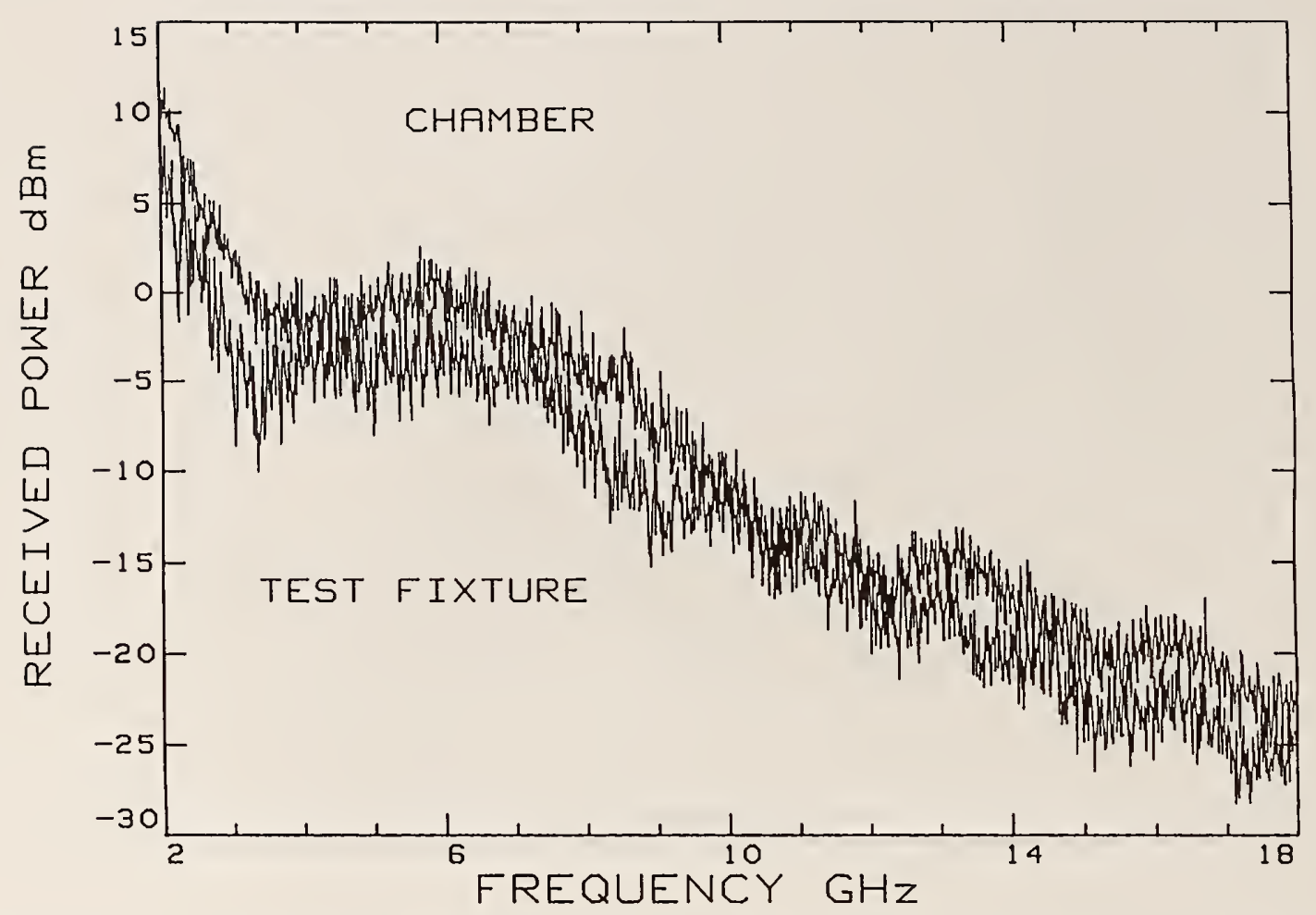

Figure 4-53. Comparison of baseline data collected from the test fixture and from the reverberation chamber.

SHIELDING OF WINDOW USING BLWGN METHOD

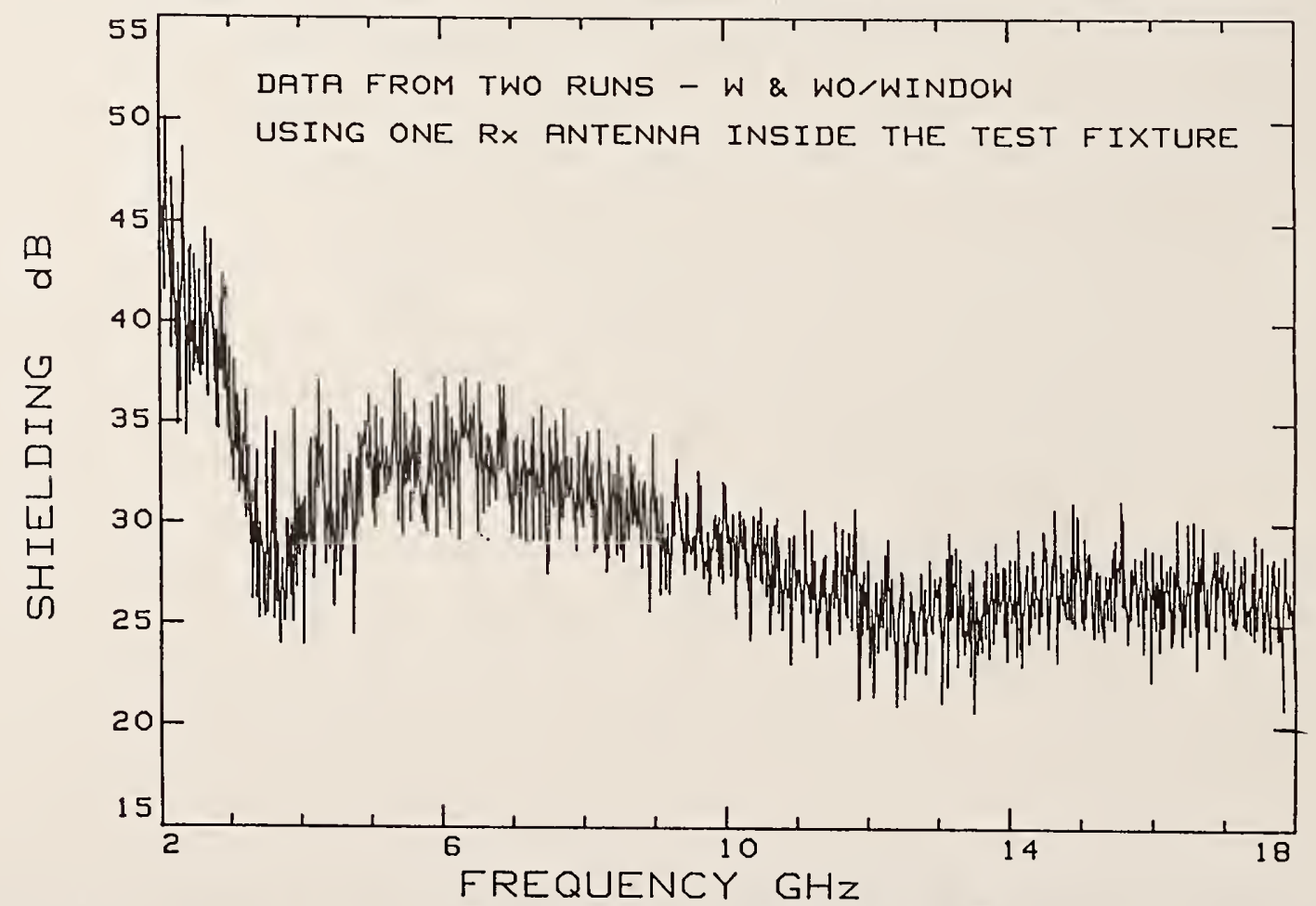

Figure 4-54. Results of window SE measurements made using BLWGN excitation with and without window mounted to the test fixture. 
SHIELDING OF WINDOW USING MODE STIR METHOD

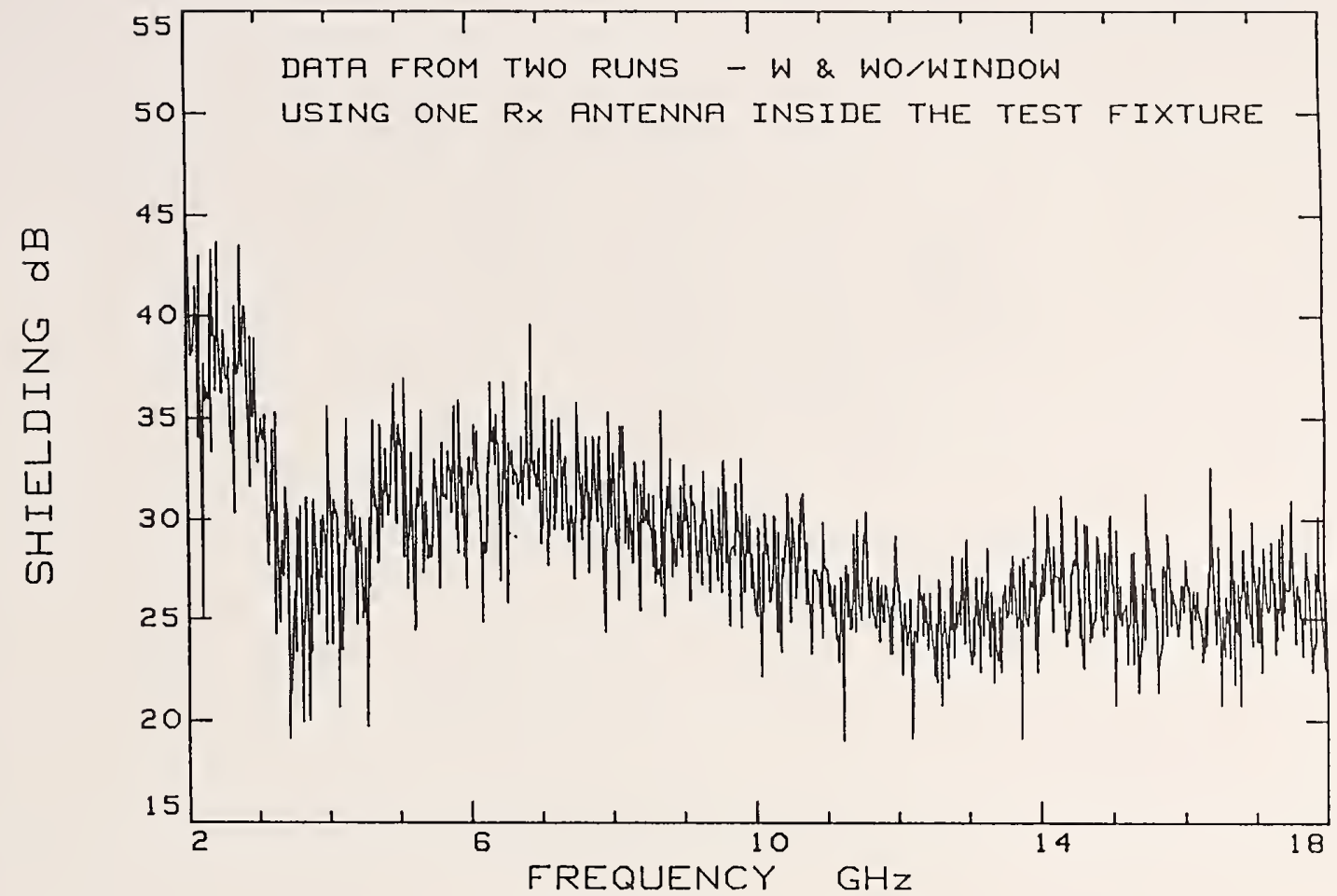

Figure 4-55. Results of window SE measurements made using CW (mechanical mode mixing) excitation with and without window mounted to the test fixture.

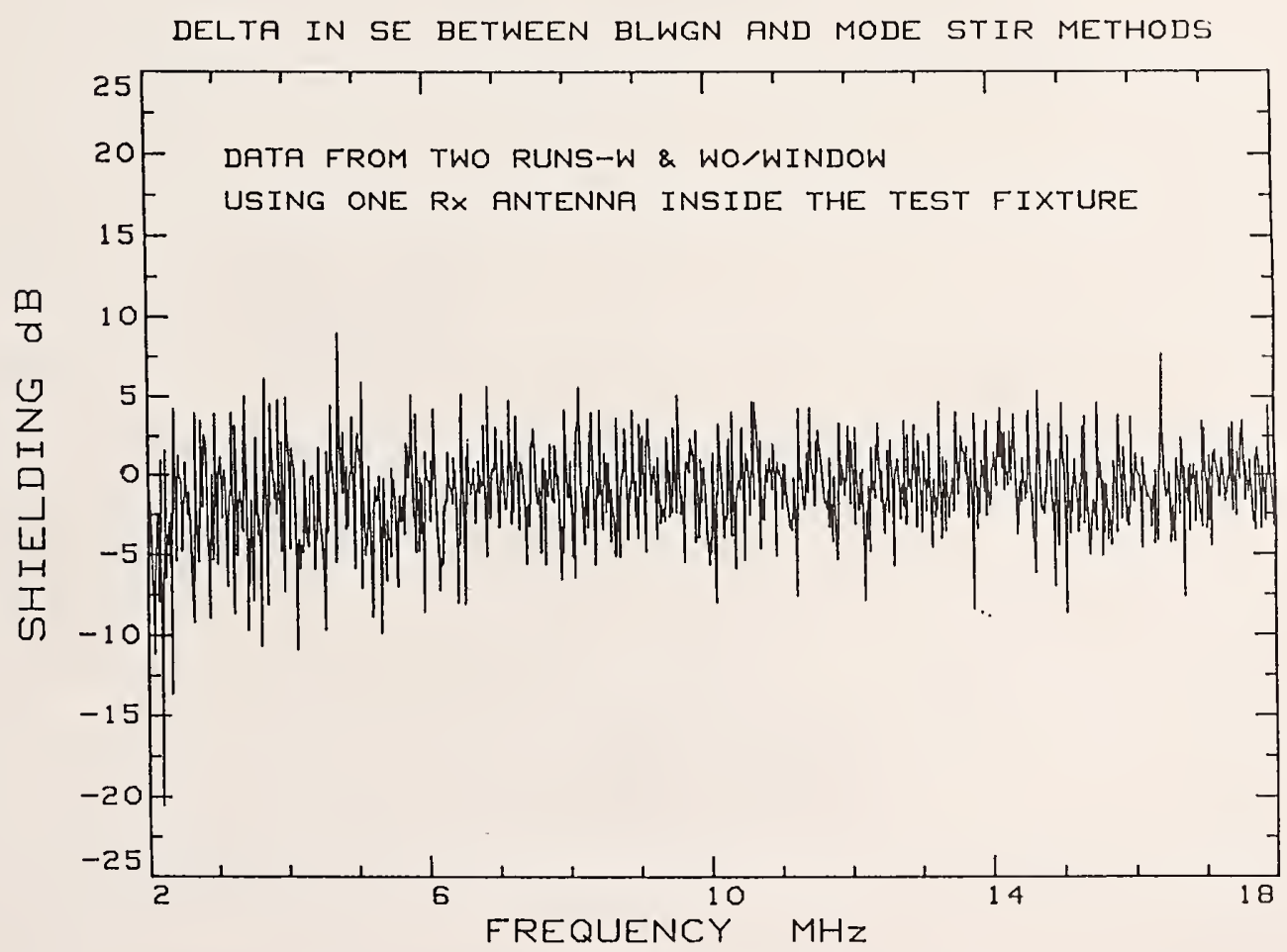

Figure 4-56. Difference in SE obtained using BLWGN and mechanical mode mixing. 


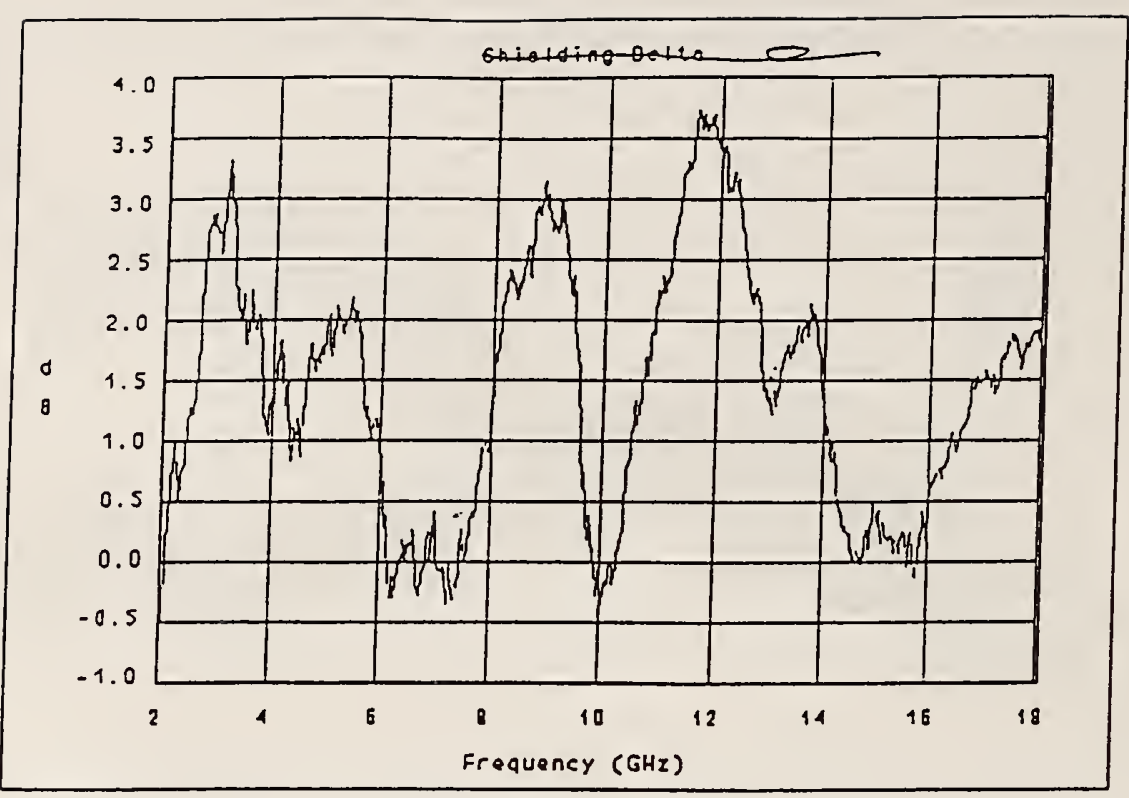

Figure 4-57. Difference in shielding after smoothing.

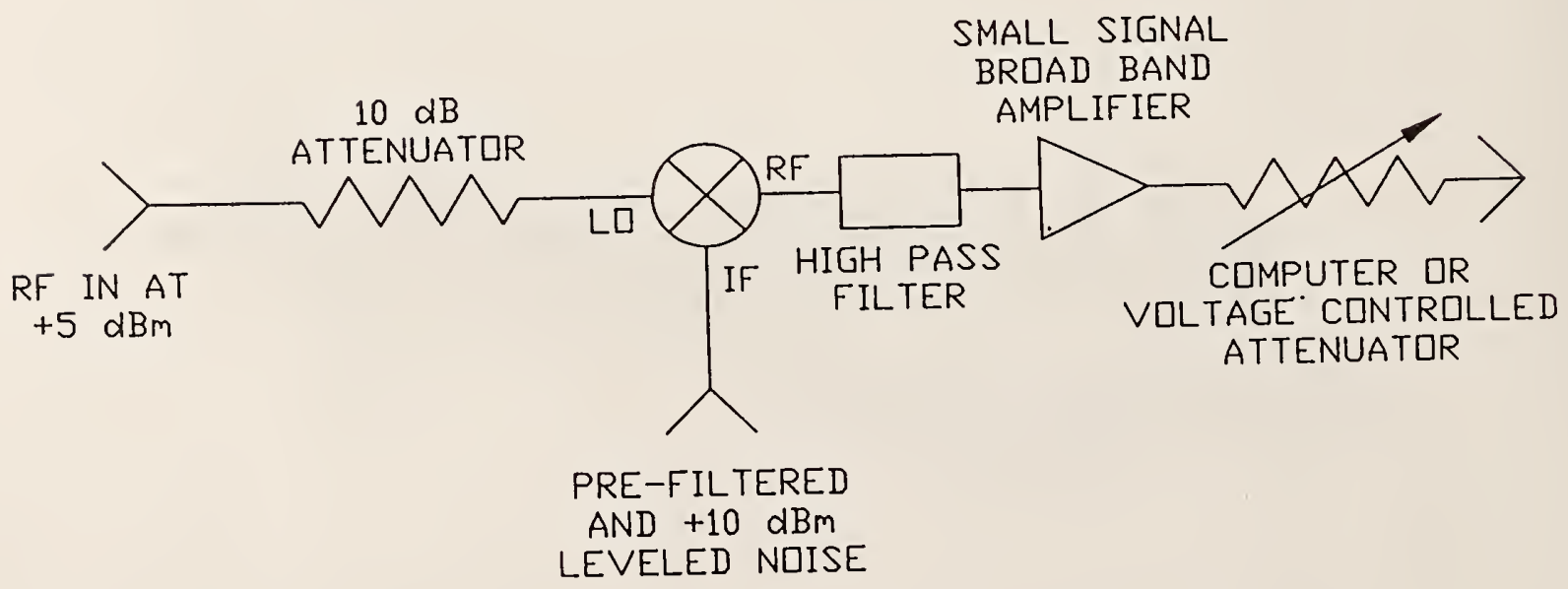

Figure 5-1. Improved modulator configuration. 




\section{NIST Technical Publications}

\section{Periodical}

Journal of Research of the National Institute of Standards and Technology-Reports NIST research and development in those disciplines of the physical and engineering sciences in which the Institute is active. These include physics, chemistry, engineering, mathematics, and computer sciences. Papers cover a broad range of subjects, with major emphasis on measurement methodology and the basic technology underlying standardization. Also included from time to time are survey articles on topics closely related to the Institute's technical and scientific programs. Issued six times a year.

\section{Nonperiodicals}

Monographs-Major contributions to the technical literature on various subjects related to the Institute's scientific and technical activities.

Handbooks-Recommended codes of engineering and industrial practice (including safety codes) developed in cooperation with interested industries, professional organizations, and regulatory bodies.

Special Publications-Include proceedings of conferences sponsored by NIST, NIST annual reports, and other special publications appropriate to this grouping such as wall charts, pocket cards, and bibliographies.

Applied Mathematics Series-Mathematical tables, manuals, and studies of special interest to physicists, engineers, chemists, biologists, mathematicians, computer programmers, and others engaged in scientific and technical work.

National Standard Reference Data Series-Provides quantitative data on the physical and chemical properties of materials, compiled from the world's literature and critically evaluated. Developed under a worldwide program coordinated by NIST under the authority of the National Standard Data Act (Public Law 90-396). NOTE: The Journal of Physical and Chemical Reference Data (JPCRD) is published bimonthly for NIST by the American Chemical Society (ACS) and the American Institute of Physics (AIP). Subscriptions, reprints, and supplements are available from ACS, 1155 Sixteenth St., NW, Washington, DC 20056.

Building Science Series-Disseminates technical information developed at the Institute on building materials, components, systems, and whole structures. The series presents research results, test methods, and performance criteria related to the structural and environmental functions and the durability and safety characteristics of building elements and systems.

Technical Notes-Studies or reports which are complete in themselves but restrictive in their treatment of a subject. Analogous to monographs but not so comprehensive in scope or definitive in treatment of the subject area. Often serve as a vehicle for final reports of work performed at NIST under the sponsorship of other government agencies.

Voluntary Product Standards-Developed under procedures published by the Department of Commerce in Part 10, Title 15, of the Code of Federal Regulations. The standards establish nationally recognized requirements for products, and provide all concerned interests with a basis for common understanding of the characteristics of the products. NIST administers this program in support of the efforts of privatesector standardizing organizations.

Consumer Information Series-Practical information, based on NIST research and experience, covering areas of interest to the consumer. Easily understandable language and illustrations provide useful background knowledge for shopping in today's technological marketplace.

Order the above NIST publications from: Superintendent of Documents, Government Printing Office, Washington, DC 20402.

Order the following NIST publications-FIPS and NISTIRs-from the National Technical Information Service, Springfield, VA 22161.

Federal Information Processing Standards Publications (FIPS PUB)-Publications in this series collectively constitute the Federal Information Processing Standards Register. The Register serves as the official source of information in the Federal Government regarding standards issued by NIST pursuant to the Federal Property and Administrative Services Act of 1949 as amended, Public Law 89-306 (79 Stat. 1127), and as implemented by Executive Order 11717 (38 FR 12315, dated May 11, 1973) and Part 6 of Title 15 CFR (Code of Federal Regulations).

NIST Interagency Reports (NISTIR)-A special series of interim or final reports on work performed by NIST for outside sponsors (both government and non-government). In general, initial distribution is handled by the sponsor; public distribution is by the National Technical Information Service, Springfield, VA 22161, in paper copy or microfiche form. 
U.S. Department of Commerce

National Institute of Standards and Technology

325 Broadway

Boulder, Colorado 80303-3328

Official Business

Penalty for Private Use, $\$ 300$ 